\title{
Next-Generation Ultrafast Transmission Electron Microscopy - Development and Applications
}

\section{DISSERTATION}

zur Erlangung des mathematisch-naturwissenschaftlichen Doktorgrades

"Doctor rerum naturalium"

der Georg-August-Universität Göttingen

im Promotionsprogramm ProPhys

der Georg-August University School of Science (GAUSS)

von

Herrn Armin Feist

aus Wolmirstedt

Göttingen, 2018 


\section{Betreuungsausschuss}

Prof. Dr. Claus Ropers, IV. Physikalisches Institut

Prof. Dr. Konrad Samwer, I. Physikalisches Institut

Prof. Dr. Sascha Schäfer, Institut für Physik, Carl von Ossietzky Universität, Oldenburg

Mitglieder der Prüfungskommission

Referent: $\quad$ Prof. Dr. Claus Ropers, IV. Physikalisches Institut

Korreferent: $\quad$ Prof. Dr. Tim Salditt, Institut für Röntgenphysik

2. Korreferent: $\quad$ PD Dr. Klaus Sokolowski-Tinten,

Fakultät für Physik, Universität Duisburg-Essen

Weitere Mitglieder der Prüfungskommission

Prof. Dr. Fabian Heidrich-Meisner, Institut für Theoretische Physik

Prof. Dr. Christian Jooß, Institut für Materialphysik

Prof. Dr. Konrad Samwer, I. Physikalisches Institut

Prof. Dr. Alec Wodtke, Institut für Physikalische Chemie

Tag der mündlichen Prüfung: 05. Juni 2018 
The great advances in science usually result from new tools rather than from new doctrines.

Freeman Dyson 



\section{Abstract}

The present cumulative thesis covers the development and applications of a novel type of ultrafast transmission electron microscope (UTEM) employing high-coherence electron pulses from a nanoscale photocathode.

Specifically, a commercial Schottky field-emission TEM is modified to allow for ultrashort electron pulse generation by photoemission from a nanoscopic field emission tip. At the sample position, electron focal spot sizes down to $9 \AA$, an electron pulse duration of $200 \mathrm{fs}$ (full-width at half-maximum) and a spectral bandwidth of $0.6 \mathrm{eV}$ are demonstrated. The instrumental capabilities for ultrafast electron diffraction, imaging and spectroscopy are illustrated.

A first detailed experiment harnesses the coherent quantum state manipulation of freeelectron wavefunctions. The kinetic energy distribution of high-energy electrons traversing an intense optical near-field evolves into a comb of spectral sidebands spaced by the photon energy. Multilevel Rabi oscillations are observed in the optical-field dependent sideband populations, experimentally demonstrating the preparation of a coherent longitudinal momentum superposition state. Numerical simulations verify the formation of an attosecond electron pulse train after dispersive propagation.

In a second study, the optically-induced ultrafast structural dynamics close to the edge of a single crystalline graphite membrane are investigated with a $28-\mathrm{nm} / 700$-fs spatio-temporal resolution. Ultrafast convergent beam electron diffraction (U-CBED) is established as a quantitative technique to access the local lattice deformations on the femtosecond timescale. The complex acoustic distortions are disentangled by reconstruction of the relevant deformation gradient tensor components. Lateral scanning of the electron probe tracks the excitation, propagation and dissipation of the optically induced expansion and shear deformations.

\section{Zusammenfassung}

Die vorliegende kumulative Dissertation befasst sich mit der Entwicklung und Anwendung eines neuartigen ultraschnellen Transmissionselektronenmikroskops (UTEM), welches hochkohärente Elektronenpulse aus einer nanoskaligen Photokathode nutzt.

Insbesondere wird ein kommerzielles Schottky-Feldemissions-TEM modifiziert, um die Erzeugung ultrakurzer Elektronenpulse durch Photoemission von einer nanoskopischen 
Feldemissionsspitze zu ermöglichen. An der Probenposition werden Elektronenfokusgrößen bis hinunter zu $9 \AA$, eine Elektronenpulsdauer von 200 fs (Halbwertsbreite) und eine spektrale Bandbreite von $0.6 \mathrm{eV}$ demonstriert. Weiterhin werden die instrumentellen Fähigkeiten für ultraschnelle Elektronenbeugung, Bildgebung und Spektroskopie veranschaulicht.

Ein erstes detailliertes Experiment realisiert die kohärente Quantenzustandsmanipulation der Wellenfunktionen freier Elektronen. Die kinetische Energieverteilung hochenergetischer Elektronen, welche ein intensives optisches Nahfeld durchqueren, entwickelt sich zu einem Kamm aus spektralen Seitenbändern, mit der Photonenenergie als Abstand. Multi-Niveau Rabi-Oszillationen werden in der optischen Feldstärkeabhängigkeit der Seitenbandpopulationen beobachtet, was experimentell die Präparation eines kohärenten Longitudinalimpuls-Superpositionszustands demonstriert. Numerische Simulationen bestätigen die Erzeugung eines Attosekunden-Elektronenpulszuges nach dispersiver Propagation.

In einer zweiten Studie wurde die optisch induzierte ultraschnelle Strukturdynamik nahe der Kante einer einkristallinen Graphitmembran mit einer 28-nm/700-fs raum-zeitlichen Auflösung untersucht. Ultraschnelle konvergente Elektronenbeugung (U-CBED) wird als quantitative Methode etabliert um auf die lokale Gitterdeformation auf FemtosekundenZeitskalen zuzugreifen. Die komplexen akustischen Verzerrungen werden durch Rekonstruktion der relevanten Komponenten des Deformationsgradienten aufgelöst. Die laterale Abtastung durch den Elektronenfokus ermöglicht die Verfolgung von Anregung, Ausbreitung und Dissipation der optisch angeregten Expansions- und Scherdeformationen. 


\section{Contents}

List of Figures $\quad$ xi

Symbols and Abbreviations xiii

1 Introduction 1

2 Ultrafast electron microscopy and diffraction 5

2.1 Transmission electron microscopy . . . . . . . . . . . . . . . 6

2.2 Elements of ultrafast electron diffraction and microscopy . . . . . . . . 14

3 Properties and generation of ultrashort electron pulses 21

3.1 Phase space description of electron beams . . . . . . . . . . . . . . . 21

3.2 Time evolution of phase space distributions . . . . . . . . . . . . 27

3.3 Generation of free-electron beams . . . . . . . . . . . . . . . . . . 29

4 Ultrafast transmission electron microscopy using a laser-driven field ... $\quad 39$

4.1 Introduction . . . . . . . . . . . . . . . . . . . . . . 40

4.2 Instrumentation . . . . . . . . . . . . . . . . . 42

4.3 Implementation of a laser-triggered field emitter in the UTEM . . . . . . 44

4.3.1 Localized photoemission from needle-shaped photocathodes . . . 44

4.3.2 Characterization of spatial beam properties using photoelectrons . 46

4.3.3 Characterization of temporal electron bunch properties . . . . . . 49

4.4 Selected applications . . . . . . . . . . . . . . . 52

4.5 Optical interactions with free-electron beams in the field-emitter UTEM . 55

4.6 Conclusion and outlook . . . . . . . . . . . . . . 58

5 Quantum coherent optical phase modulation in an ultrafast transmission ... 61

5.1 Introduction . . . . . . . . . . . . . . . . . . . . 62

5.2 Main part ......................... 62 
5.3 Conclusion $\ldots \ldots \ldots \ldots \ldots \ldots \ldots \ldots$

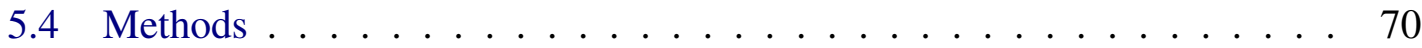

5.4.1 Ultrafast TEM and experimental setup . . . . . . . . . . . 70

5.4.2 Temporal characterization of electron pulses _ . . . . . . 72

5.4.3 Data analysis and drift correction $\ldots \ldots \ldots \ldots . \ldots 73$

5.4 .4 Materials . . . . . . . . . . . . . . . . . . 75

5.4.5 Quantum description using ladder operators . . . . . . . . . 75

5.4 .6 Numerical calculations . . . . . . . . . . . . . . . . 80

6 Nanoscale diffractive probing of strain dynamics in ultrafast ...

6.1 Introduction . . . . . . . . . . . . . . . . . . . 86

6.2 Ultrafast convergent beam electron diffraction . . . . . . . . . . 87

6.3 Extracting the deformation gradient tensor $\ldots \ldots \ldots \ldots \ldots$

6.4 Local ultrafast structural dynamics . . . . . . . . . . . . . . . . . . 94

6.5 Spatio-temporal strain mapping . . . . . . . . . . . . . . . . 96

6.6 Transient Bragg line profiles . . . . . . . . . . . . . . . . . 98

6.7 Conclusion . . . . . . . . . . . . . . . . . . . . . . . . . 100

6.8 Supplementary material . . . . . . . . . . . . . . . . . . . 100

6.8.1 Ultrafast convergent beam electron diffraction (U-CBED) _ . . 100

6.8 .2 Sample . . . . . . . . . . . . . . . . . . . . 102

6.8.3 Data collection \& analysis . . . . . . . . . . . . 102

6.8.4 Debye Waller analysis . . . . . . . . . . . . . . . . . . 103

6.8.5 Numerical simulation of graphite thin film lattice dynamics . . . 105

6.8.6 Characterization of the optical near-field structure at the ... . . . 106

7 Discussion 109

7.1 Ultrafast TEM using coherent electron pulses _ . . . . . . . . . . . 109

7.1.1 Comparison of ultrafast electron gun concepts . . . . . . . . . 110

7.1.2 Considerations for future UTEM instruments . . . . . . . . 113

7.2 Quantum coherent control of free-electron beams . . . . . . . . . . 117

7.2.1 Coherent control of free-electron wave functions by light . . . . 117

7.2.2 Generation of attosecond electron pulse trains . . . . . . . . 118

7.2.3 A three-dimensional all-optical electron phase plate . . . . . . . 120 
7.3 Probing ultrafast nanoscale dynamics in UTEM . . . . . . . . . . . 120

7.3.1 Time-domain access to nanophononic systems . . . . . . . . 121

7.3.2 Methods for studying ultrafast nanoscale dynamics . . . . . . . . 123

7.4 Conclusion \& further perspectives . . . . . . . . . . . . . . . . . 126

$\begin{array}{lr}\text { Bibliography } & 129\end{array}$

Author contributions, publications and conference talks 171

$\begin{array}{ll}\text { Danksagung } & 175\end{array}$ 



\section{List of Figures}

2.1 Transmission electron microscopy. . . . . . . . . . . . . . . 6

2.2 Electron-sample interactions in TEM. . . . . . . . . . . . . . . 8

2.3 Transverse phase manipulation of free-electron beams. . . . . . . . . . . 9

2.4 Nanoscale strain analysis in TEM. . . . . . . . . . . . . . . . . . . 12

2.5 Ultrafast electron diffraction (UED) in transmission. . . . . . . . . . . 15

2.6 Ultrafast electron diffraction in reflection. . . . . . . . . . . . . . . 16

2.7 Imaging strain-stress dynamics in UTEM. . . . . . . . . . . . . . . . 17

2.8 Ultrafast local diffractive probing in UTEM. . . . . . . . . . . . . . 18

2.9 Inelastic electron light scattering (IELS) in optical near-fields. . . . . . . 19

2.10 Photon-induced near-field electron microscopy (PINEM). . . . . . . . . . 20

3.1 Properties of single-electron pulses. . . . . . . . . . . . . . 23

3.2 Temporal evolution of phase space distributions. . . . . . . . . . . . . 28

3.3 Concept of longitudinal RF-compression. . . . . . . . . . . . . . . . . . 29

3.4 Processes of electron emission from solids. . . . . . . . . . . . . . 30

3.5 Emitter geometries of continuous electron sources. . . . . . . . . . . 31

3.6 Potential landscape of an electron emitter. . . . . . . . . . . . . . . . 32

3.7 Photoemission processes from solids. . . . . . . . . . . . . . . 36

4.1 Schematic setup and electron pulse properties of the Göttingen UTEM. . . 43

4.2 Transverse beam properties of photoelectron beams emitted from lasertriggered electron sources. . . . . . . . . . . . . . . . . 44 45

4.3 Operation principle of a laser driven Schottky field emitter and its geometry. 46

4.4 Characterization of the transverse electron beam properties. . . . . . . . . 48

4.5 Temporal pulse characterization. . . . . . . . . . . . . . . . 52

4.6 Applications of low-emittance electron pulses in ultrafast electron imaging. 53

4.7 Exemplary experimental results achievable with the current status of the UTEM instrument. . . . . . . . . . . . . . . . . . . . . 54 
4.8 Applications of quantum coherent electron light interactions in optical near-fields within an ultrafast TEM. . . . . . . . . . . . . . . . 57

5.1 Schematic and principles of coherent inelastic electron scattering by optical near-fields. . . . . . . . . . . . . . . . . . 64

5.2 Quantum coherent manipulation of electron energy distributions. . . . . . 67

5.3 Formation of an attosecond electron pulse train. . . . . . . . . . . . . . 69

5.4 Experimental setup. . . . . . . . . . . . . . . . . . . . 71

5.5 Spatial characterization of near-field scattering. . . . . . . . . . . 72

5.6 Pulse characterization by electron-photon cross-correlation. . . . . . . . 73

5.7 Evaluation of sideband populations. . . . . . . . . . . . . . . 74

5.8 Influence of spatial and temporal averaging. . . . . . . . . . . . . 81

5.9 Robustness of attosecond pulse train generation. . . . . . . . . . . . 82

6.1 Ultrafast convergent beam electron diffraction on single crystalline graphite. 89

6.2 Transient modulation of Bragg line profiles. . . . . . . . . . . . . . 90

6.3 Time-dependent Bragg-line changes and dynamics of selected components of the deformation gradient tensor. . . . . . . . . . . . . . . . . . . 93

6.4 Spatio-temporal mapping of near-edge strain dynamics. . . . . . . . . . . 97

6.5 Dynamics of the (422) Bragg line profile. . . . . . . . . . . . . . . . 99

6.6 Experimental setup. . . . . . . . . . . . . . . . . . . 101

6.7 Evaluation of the diffraction data. . . . . . . . . . . . . 103

6.8 Intensity change of the (452) Bragg line. . . . . . . . . . . . . . . 105

6.9 Characterization of the optical near-field at the graphite edge. . . . . . . . 107

7.1 Comparison of ultrafast electron gun concepts. . . . . . . . . . . . . 111

7.2 Electron pulse compression using THz-fields. . . . . . . . . . . . . . . 115

7.3 Control of nanotip photoemission with terahertz pulses. . . . . . . . . 116

7.4 Coherent control of free-electron beams. . . . . . . . . . . . . . . . 118

7.5 Generation and reconstruction of attosecond electron pulse trains in UTEM.119

7.6 Transverse effects in IELS . . . . . . . . . . . . . . . . . . . . 121

7.7 Nanoscale optomechanical crystal. . . . . . . . . . . . . . . . . . . 122

7.8 Time and length scales addressed by fast probing techniques. . . . . . . 125

7.9 Prospects of coherent UTEM. . . . . . . . . . . . . . . . . . . . 127 


\section{Symbols and Abbreviations}

\begin{tabular}{|c|c|c|}
\hline Symbol & Meaning & Unit \\
\hline$a, a^{\dagger}$ & lowering and raising operators & \\
\hline $\mathbf{a}_{i}$ & real space basis vector & $\mathrm{nm}$ \\
\hline $\mathbf{A}$ & magnetic vector potential & $\mathrm{Vs} \mathrm{m}^{-1}$ \\
\hline $\mathbf{b}_{i}, \mathbf{B}$ & reciprocal space vector and basis & $\mathrm{nm}^{-1}$ \\
\hline$B_{n}, B_{n p}$ & normalized (peak) brightness & $\mathrm{Am}^{-2} \mathrm{sr}$ \\
\hline$c$ & vacuum speed of light & $\mathrm{ms}^{-1}$ \\
\hline$e$ & elementary charge & $\mathrm{C}$ \\
\hline$E_{0}, E, E_{\mathrm{F}}, \Delta E$ & (initial) kinetic energy, Fermi energy, energy spread & $\mathrm{eV}$ \\
\hline$f$ & Fermi-Dirac distribution & 1 \\
\hline$F$ & electric field & $\mathrm{Vnm}^{-1}$ \\
\hline $\mathbf{F}$ & deformation gradient tensor & 1 \\
\hline $\mathcal{F}$ & Fourier Transform & \\
\hline$g$ & coupling constant & 1 \\
\hline$\hbar$ & reduced Planck constant & $\mathrm{J} \mathrm{s}$ \\
\hline$I$ & intensity & $\mathrm{GW} \mathrm{cm}^{-2}$ \\
\hline$j$ & current density & $\mathrm{Am}^{-2}$ \\
\hline$J$ & current & A \\
\hline$k, \mathbf{k}$ & angular wavenumber, wave vector, scattering vector & $\mathrm{m}^{-1}$ \\
\hline$k_{\mathrm{B}}$ & Boltzmann's constant & $\mathrm{JK}^{-1}$ \\
\hline$K$ & degree-of-coherence & 1 \\
\hline$m_{e}$ & electron mass & $\mathrm{kg}$ \\
\hline$N$ & sideband number/photon order & 1 \\
\hline$N_{e},\left\langle N_{e}\right\rangle$ & (mean) number of electrons (per pulse) & 1 \\
\hline$p_{0}, p, \mathbf{p},\left\langle p_{i}{ }^{2}\right\rangle$ & (initial) momentum (operator) & $\mathrm{kg} \mathrm{ms}^{-1}$ \\
\hline$q_{i},\left\langle q_{i}^{2}\right\rangle$ & position (operator) & $\mathrm{m}$ \\
\hline$Q$ & charge & As \\
\hline
\end{tabular}




\begin{tabular}{|c|c|c|}
\hline$r_{0}, r, \mathbf{r}$ & (tip) radius, distance, position vector & $\mathrm{nm}$ \\
\hline$\Delta r$ & change in (angular) radius & $\operatorname{mrad}$ \\
\hline$R, \mathbf{R}$ & rotation tensor & 1 \\
\hline $\mathbf{R}$ & lattice vector & $\mathrm{nm}$ \\
\hline$t, \Delta t$ & time, time delay & fs \\
\hline$T$ & (optical) period & fs \\
\hline$T_{0}, \Delta T$ & temperature rise, base temperature & $\mathrm{K}$ \\
\hline$u, \mathbf{u}$ & displacement, displacement field & $\mathrm{nm}$ \\
\hline$U_{0}$ & acceleration voltage & $\mathrm{V}$ \\
\hline$V, \bar{V}$ & electrostatic potential, averaged lattice potential & $\mathrm{eV}$ \\
\hline$v, v_{e}$ & velocity, relativistic electron velocity & $\mathrm{ms}^{-1}$ \\
\hline$x, y, z$ & spatial coordinates & $\mathrm{nm}$ \\
\hline$\alpha$ & convergence angle & mrad \\
\hline$\gamma$ & Lorentz factor & 1 \\
\hline$\varepsilon_{\mathrm{n}, \mathrm{rms}}, \varepsilon_{0}$ & normalized rms emittance, quantum emittance & nmmrad \\
\hline$\varepsilon, \varepsilon$ & strain tensor (component) & 1 \\
\hline$\theta_{\mathrm{B}}, \Delta \theta$ & (Bragg) angle, diffraction angle & mrad \\
\hline$\lambda, \lambda_{e}$ & (electron) wavelength & $\mathrm{nm}$ \\
\hline$\xi_{c, x / t}$ & transverse/temporal coherence length & \\
\hline$\xi$ & scattering extinction lengths & $\mathrm{nm}$ \\
\hline$\rho$ & density (operator) & 1 \\
\hline$\sigma$ & (mechanical) stress & $\mathrm{Nm}^{2}$ \\
\hline$\sigma_{q_{i} / p_{i} / x / \alpha}$ & $\begin{array}{l}\text { standard deviation of position/momentum, } \\
\text { beam diameter/angular distribution }\end{array}$ & \\
\hline$\tau$ & pulse duration, time constant & fs \\
\hline$\varphi, \Delta \varphi(h k l)$ & relative phase/angle & $\mathrm{rad}$ \\
\hline$\phi_{e}$ & electron phase & 1 \\
\hline$\Phi_{\mathrm{W}}$ & work function & $\mathrm{eV}$ \\
\hline$\Phi$ & electrostatic potential & $\mathrm{V}$ \\
\hline$\omega$ & optical (angular) frequency & $\mathrm{Hz}$ \\
\hline$\omega, \omega$ & rotation tensor (component) & 1 \\
\hline$\psi$ & wavefunction & 1 \\
\hline$\Omega^{6 \mathrm{D}}$ & phase space degeneracy & 1 \\
\hline
\end{tabular}




\begin{tabular}{|c|c|}
\hline Abbreviation & Meaning \\
\hline CBED & convergent beam electron diffraction \\
\hline CL & cathodoluminescence \\
\hline $\mathrm{cW}$ & continuous wave \\
\hline DTEM & dynamic transmission electron microscope \\
\hline $\operatorname{EEL}(\mathrm{S})$ & electron energy loss (spectroscopy) \\
\hline FEL & free-electron laser \\
\hline FWHM & full-width at half-maximum \\
\hline HHG & high harmonic generation \\
\hline HRTEM & high resolution electron microscopy \\
\hline IELS & inelastic electron-light scattering \\
\hline LIED & laser-induced electron diffraction \\
\hline PINEM & photon-induced near-field electron microscopy \\
\hline PPM & point-projection microscopy \\
\hline $\mathrm{RF}$ & radio-frequency \\
\hline rms & root-mean-square \\
\hline ROI & region of interest \\
\hline S-PINEM & scanning photon-induced near-field electron microscopy \\
\hline \multirow[t]{2}{*}{ SQUIRRELS } & spectral quantum interference for the regularised \\
\hline & reconstruction of free-electron states \\
\hline STEM & scanning transmission electron microscopy \\
\hline STM & scanning tunneling microscopy \\
\hline $\mathrm{THz}$ & terahertz \\
\hline trARPES & time and angle resolved photoemission spectroscopy \\
\hline TR-PEEM & time-resolved photoemission electron microscopy \\
\hline U-CBED & ultrafast convergent beam electron diffraction \\
\hline UED & ultrafast electron diffraction \\
\hline UEM & ultrafast electron microscopy \\
\hline U-LEED & (ultrafast) low-energy electron diffraction \\
\hline U-RHEED & (ultrafast) reflection-high-energy electron diffraction \\
\hline UTEM & ultrafast transmission electron microscopy/microscope \\
\hline ZLP & zero-loss peak \\
\hline
\end{tabular}





\section{Chapter 1}

\section{Introduction}

At the heart of natural science lies the curiosity to comprehend the composition and function of natural objects and phenomena as they evolve with time. The centuries of striving for new insights gave rise to the development of scientific instruments resolving the structure and dynamics of matter on length and time scales exceeding those of human perception by orders of magnitude.

Our prevalent method for studying nature is by visible light. Until the beginning of the 19th century, optical microscopes have provided for imaging with $\mu \mathrm{m}$-spatial, diffraction limited resolution [1], and photography with fast shutter speeds could obtain sub-ms snapshots of dynamic scenes [2]. Diffraction techniques, revealing structural periodicity, enabled the exploration of atomic lattices using short wavelength X-rays [3], and later electron beams in vacuum [4].

The transmission electron microscope (TEM) [5] provided real-space imaging capability of structural inhomogeneities, quickly surpassing optical microscopes and advancing to an atomic-scale resolution [6]. Most advances of the TEM methodology were propelled by instrumental breakthroughs, like aberration correction [7-10], new detectors [11], tomography [12], cryo-EM [13], energy filtered [14] and monochromated TEM [15]. Especially, the availability of high-brightness electron beams facilitated the development of advanced electron holography [16] and coherent beam shaping techniques [17].

In the realm of optics, shifting from fast image acquisition to stroboscopic illumination, the achievable temporal resolution is only limited by the shortest flashes of light available. Driven by the rapid development of coherent light-sources [18, 19], the study of dynamic processes could be drastically extended from the nanosecond regime [20] to pico- [21], femto- [22] and nowadays attosecond [23] timescales. 
Of central interest in physics and materials science are the ultrafast dynamics in condensed matter, especially those of nanostructured systems, as relevant for basically all high-technological devices. That includes a variety of processes, e.g. the ultrafast energy dissipation and transfer at and across interfaces, the observation of ultrafast phase transformations, ultrafast magnetization dynamics and the transient response of solids excited by intense optical fields.

Physical processes occurring in extended volumes obey a natural hierarchy of time and length scales. The intrinsic velocities of particle diffusion, flow of charge, propagating collective excitations, and ultimately light dictate faster dynamics to occur on shorter length scales. The clear mismatch of techniques, mainly addressing the spatial or temporal dimensions of dynamic processes, demands for new instrumental developments.

Using femtosecond probe pulses, ultrafast electron [24, 25] and X-ray [26] diffraction techniques yield insight into fundamental processes in spatially homogeneous samples, with excellent resolution in Fourier space. Near-field scanning [27, 28] and local emission microscopes [29] could study ultrafast dynamics in real-space, with typical geometric experimental constraints applying.

After early work done at the Technical University of Berlin [30], the field of timeresolved TEM was advanced by the implementations of nanosecond single-shot dynamic TEM (DTEM) at Lawrence Livermore National Laboratory [31], and with nanosecond to sub-picosecond time-resolution by stroboscopic illumination in the group of A. H. Zewail at Caltech [32].

This thesis contributes to the rapidly evolving field of ultrafast transmission electron microscopy (UTEM) by introducing high-coherence ultrashort electron pulses. A novel type of photoelectron source, based on laser-triggered electron emission from nanoscale metal tips, is integrated into a commercial TEM and various applications are illustrated. The unique electron pulse properties are harnessed for the exploration of two original research directions in UTEM. Firstly, the quantum coherent optical phase modulation of free-electron beams is experimentally demonstrated, opening up new avenues in the coherent control and shaping of electron wavefunctions. Secondly, ultrafast convergent beam electron diffraction accesses the ultrafast structural dynamics in nanoscale sample volumes, enabling the study of nanophononic systems on their intrinsic picosecond time and nanometer length scale. 


\section{Outline}

This cumulative thesis is composed of three original publications (Chapters 4-6), each describing their respective scientific relevance, the experimental setup and results, completed by a comprehensive discussion. In the following, Chapter 2 and 3 present main theoretical and experimental prerequisites connecting this thesis to previous work. Chapter 2 introduces the TEM methodology with emphasis on coherent electron interactions and showcases selected achievements of ultrafast electron diffraction and microscopy. Chapter 3 covers the theoretical description of ultrashort electron pulses in phase space, followed by methods for ultrashort electron pulse generation.

In Chapter 4, the implementation, characterization and applications of the Göttingen UTEM instrument are presented. The original publication "Ultrafast transmission electron microscopy using a laser-driven field emitter: Femtosecond resolution with a high coherence electron beam" [33] introduces the laser-driven Schottky field emitter in an Ultrafast TEM, achieving record combined properties for single-electron pulses.

Chapter 5 studies the quantized inelastic coherent scattering of free-electrons by intense optical near-fields. The manuscript "Quantum coherent optical phase modulation in an ultrafast transmission electron microscope" [34] describes the first experimental observation of Rabi oscillations in the spectral photon sidebands, being a fingerprint for the quantum coherence of this interaction. Furthermore, numerical calculations for the temporal evolution of such phase-modulated electron pulses show the generation of attosecond electron pulse trains.

Chapter 6 describes a prototypical experiment for the study of ultrafast structural dynamics in inhomogeneous systems, with simultaneous nanometer-femtosecond spatio-temporal resolution. The paper "Nanoscale diffractive probing of strain dynamics in ultrafast transmission electron microscopy" [35] applies the powerful technique of convergent beam electron diffraction (CBED) for the quantitative local probing and reconstruction of the laser-induced complex strain dynamics in a mesoscopically symmetry broken graphite thin film.

In Chapter 7, the main results of this thesis are concluded. A comparative discussion of various ultrafast electron gun concepts is given, and opportunities in the field of ultrafast TEM using high-coherence electron pulses are illustrated with focus on the optical control and structuring of free-electron beams and the study of ultrafast nanoscale dynamics. 



\section{Chapter 2}

\section{Ultrafast electron microscopy and diffraction}

Electron microscopy and diffraction are indispensable tools for resolving the structure of condensed matter and gases on short length scales. Transmission electron microscopes (TEMs) are especially refined instruments enabling versatile applications in quantitative imaging, diffraction and spectroscopy [36].

Introducing ultrashort far-field probes, ultrafast transmission electron microscopy (UTEM) provides a unique access to ultrafast dynamics in inhomogeneous systems. Starting in the 1980s, Bostanjoglo and co-workers at the Technical University of Berlin pioneered the field of "High-speed transmission electron microscopy", recording image exposure series with $\sim 200$-nm spatial resolution using $10^{8}$ electrons in $\sim 10$-ns pulses [30]. This approach using single-shot imaging to study non-reversible dynamics, later termed dynamic TEM (DTEM) was further developed at the Lawrence Livermore National Laboratory, ultimately achieving a 10-nm spatial resolution [31]. By stroboscopically illuminating reversible processes in the "single-electron"-limit, Zewail et al. could advance the UTEM methodology to the nanosecond to sub-picosecond timescales [25, 37].

Nevertheless, many quantitative TEM techniques were not accessible or severely restricted by the lack of pulsed high-brightness electron beams. Typically, the beam quality is limited by electron-electron repulsion and the large emitting area of common femtosecond electron sources.

To envisage the capabilities of UTEM, it is instrumental to briefly recapitulate the powerful tool set of conventional TEM and consider the achievements of state-of-the-art ultrafast electron diffraction and microscopy-which is the central purpose of this chapter. 
a

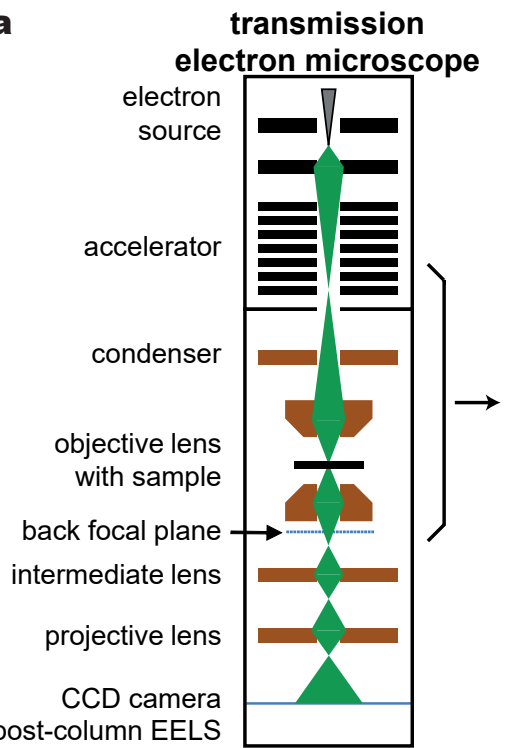

b

TEM illumination

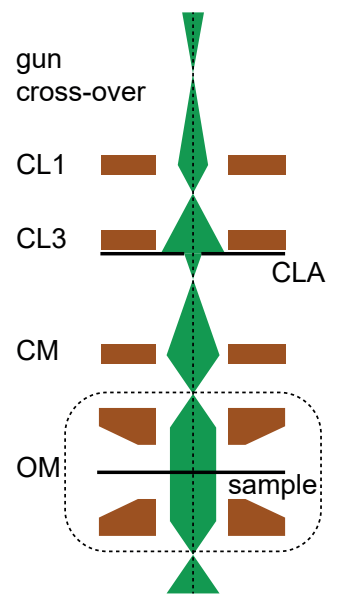

C

STEM/CB illumination

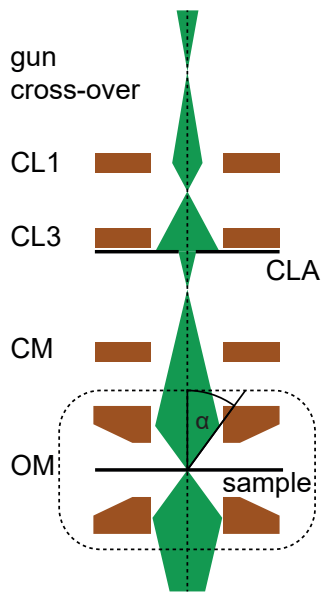

Figure 2.1: Transmission electron microscopy. (a) Schematic setup with main components of a conventional TEM. (b,c) Detailed view of the illumination system in (b) parallel and (c) convergent illumination mode. The probing beam is formed by a combination of three condenser lenses (CL1, CL3, CM), the condenser aperture (CLA) and the objective pre-field (OM, top).

\subsection{Transmission electron microscopy}

The following section briefly introduces the TEM methodology, which is covered in detail by a broad range of literature available [36, 38-43]. Special emphasis is put on coherent interactions of electrons with electromagnetic fields and matter, which are essential prerequisites for Chs. 4-6. Selected applications illustrate the opportunities of using high-coherence electron pulses in UTEM for imaging, diffraction and coherent electron beam shaping.

\section{The instrument}

Since the early days of Knoll and Ruska [5], transmission electron microscopes consist of the same principal components (cf. Fig. 2.1), with their specific counterparts found in optical microscopes. Over the years, modern TEMs added further capabilities, e.g. for holography, electron energy loss spectroscopy (EELS), scanning transmission electron microscopy (STEM), and aberration correction. 
Electron source and accelerator In an electron gun, the continuous beam generated by an electron emitter is accelerated in a high static potential, typically to $U_{0}=30-300 \mathrm{keV}$ forming a "real" or "virtual" beam crossover that acts as effective electron source. For more details, see Sec. 3.3.

Probe forming/illumination system The divergent beam from the electron gun is picked up by the condenser system, forming a well-defined, collimated or convergent electron beam. The relative lens excitations ("spot": CL1, CL3) and cutting at the variable condenser aperture (CLA) allow for tuning the beam's degree-of-coherence, adapted to the requirements of an experiment. In combination with additional lenses (CM, OL top part) the beam convergence angle ("alpha") at the sample is set. Electron optical aberrations, caused by imperfections of the emitter and lenses, can be compensated by an additional STEM probe corrector. In an advanced approach, beam preparation might also include transverse or longitudinal shaping of the electron wavefunctions (see below).

Sample Typically a thin film specimen, which is partially transparent for the electron beam (thickness: $\sim 10-200 \mathrm{~nm}$ ). After interaction with the sample, electrons, emitted photons or currents are analyzed in transmission or backscatter geometry (cf. Fig. 2.2).

Imaging system The transmitted electrons are collected by the objective lens (most often in a symmetric lens assembly housing the sample), which is usually the main source of spherical and chromatic aberrations due to the high involved scattering angles (up to $>100 \mathrm{mrad}$ ). The following system of transfer lenses magnifies either the image or back focal plane of the objective lens, enabling flexible access to the real space or reciprocal space scattering distributions. Finally, the image is projected onto an electron detector. Additional elements for aberration correction (image corrector), energy dispersion/filtering (in- or post-column) or phase analysis (e.g. an electrostatic bi-prism) might be included.

Electron detector The magnified and filtered electron distribution is recorded by following electron sensitive detectors. These include highly sensitive current detectors, like scintillator coupled photomultipliers, and lateral resolving detectors like film/plate cameras, scintillators with an attached CCD or CMOS chip and most recently, back-thinned CMOS chips for direct electron detection. In conventional TEM, the achievable timing precision 


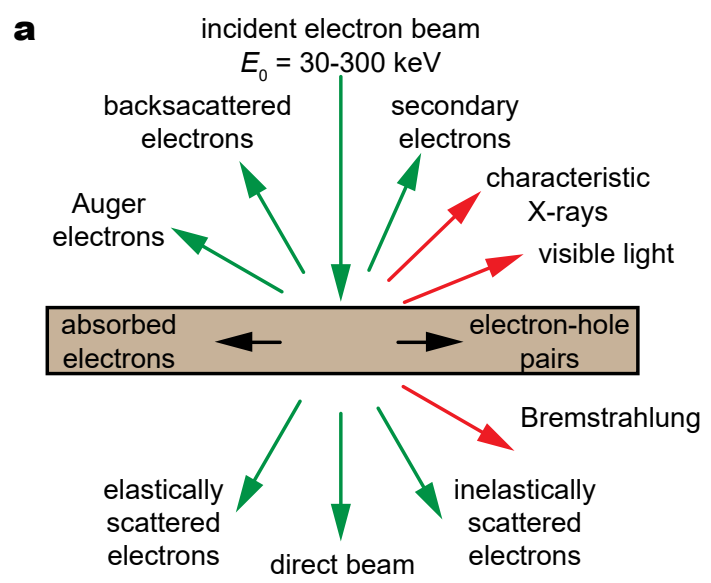

\section{b}

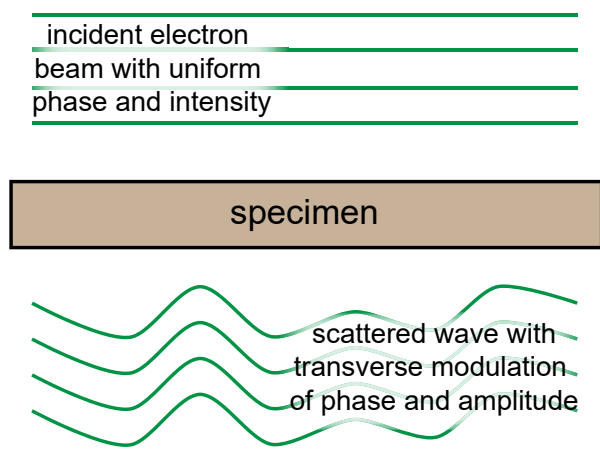

Figure 2.2: Electron-sample interactions in TEM. (a) An electron can interact with a sample in a variety of processes, creating reversible and non-reversible secondary excitation. (b) A stationary electromagnetic field configuration imprints a transverse phase-modulation onto the free-electron wavefunction (cf. Ref. [36] for more details).

is limited by the acquisition speed of the detector, e.g. for $2 \mathrm{D}$ detectors to the $\mu \mathrm{s}-$ range [44] and for 1D data down to the sub-ns regime [45].

Ultrafast TEM instruments, so far being modified traditional TEMs, make use of the same conceptional design, adding the capability for pulsed electron beams and synchronized sample excitation [25, 30-32, 46, 47]. Chapter 4 describes the instrumental design, electron beam characterization and exemplary application of the Göttingen UTEM instrument, firstly implementing high-coherence electron pulses from nanoscale photocathodes.

\section{Coherent beam interactions in TEM}

High-energy electrons interact with matter in a variety of processes, distinguishable by their action on the electron wavefunction (cf. Fig. 2.2): coherent or incoherent, elastic or inelastic and scattered in forward or backward direction. These interactions may lead to secondary processes like electron or light emission, or non-reversible changes of the sample (e.g. radiation damage).

The coherent contributions of scattering and image contrast are described in the wave picture. A wavefunction propagating along a path $\mathbf{s}$ locally accumulates a phase given by the Aharonov-Bohm equation [48]

$$
\phi_{e}=\frac{e}{\hbar}\left(\frac{1}{v_{e}} \int V(\mathbf{r}) d s-\int \mathbf{A}(\mathbf{r}) \cdot d \mathbf{s}\right),
$$



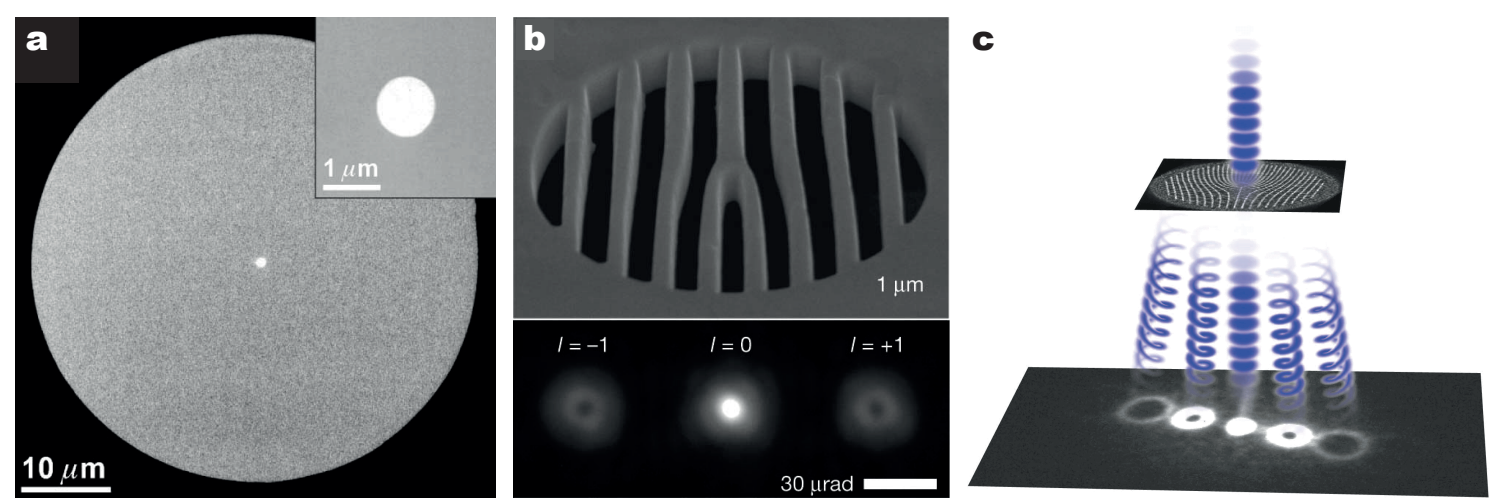

Figure 2.3: Transverse phase manipulation of free-electron beams. (a) Zernike phase plate for enhanced phase contrast imaging [51]. (b,c) Vortex beam generation by pitchfork amplitude ((b), [17]) and phase ((c), [52]) masks, creating spatially separated orbital angular momentum states. (a) Reprinted from [51], Copyright 2001, with permission from Elsevier. (b) Adapted by permission from Springer Customer Service Centre GmbH: Springer Nature, Nature [17], ( Macmillan Publishers Limited (2010). (c) From [52]. Reprinted with permission from AAAS.

with reduced Planck constant $\hbar$, electron charge $e$, velocity $v_{e}$, electrostatic potentials $V$ and magnetic vector potential $\mathbf{A}$. Passing static electromagnetic fields, either in vacuum or in a sample (e.g. mean inner atomic potentials or magnetization fields) constitutes a transverse phase modulation of the electron wavefunction.

\section{Transverse phase-structuring of electron beams}

The transverse phase manipulation of light beams is a well established technique in optical science $[49,50]$. The same principle is applied in electron microscopy, e.g. for Zernike phase plates (cf. Fig. 2.3a), which enhance the contrast of weak phase objects by adding a phase shift to the scattered part of the electron wavefunction [51]. In an advanced approach, tailored phase masks are used coherently prepare electron beams with arbitrary phase and amplitude structure.

A prominent example is the preparation of electron beams in a Laguerre-Gaussian (LG) mode, which features a spiral phase distribution and a phase singularity at its center (cf. Ref. [53] for optical equivalent). Such beams carry a quantized orbital angular momentum (OAM) $L_{z}=m \hbar$ with $m \in \mathbb{N}$ and were first realized for $m=1$ by a stack of thin graphite layers [54]. The generation of higher order OAM states is achieved by pitchfork amplitude [17] or phase gratings [52, 55], adding a linear momentum transfer for state separation (cf. Fig. 2.3b,c). Such tailored transverse electron beam states are sought after for their unique 
properties, expanding the TEM methodology, e.g. adding sensitivity for crystal chirality [56], symmetry of plasmon resonances [57] and out-of-plane magnetic fields [58].

Other implementations of elaborate phase masks are proposed for correction of spherical aberrations [59], structured illumination of samples [60] and the generation of electron Bessel beams [61], which show features of non-diffractive propagation and applications in spin filtering and polarization $[62,63]$.

The strong recent interest in phase masks illustrates the anticipated impact of tailoring the transverse electron wavefunction. Chapter 5 introduces the fundamentals of manipulating the longitudinal part of the electron wavefunction by time-varying intense optical fields, opening up a new degree of freedom in controlling free-electron beams.

\section{Coherent scattering of a crystal}

The theory of kinematic scattering assumes a single elastic scattering event, valid for thin crystals of thickness $t$ ( $t \ll \xi$, with $\xi$ is the scattering mean free path). In the weak-phaseobject (WPO) approximation, Eq. 2.1 assumes the form [43]

$$
\phi_{e}(x, y, z) \approx 1+i \pi \lambda_{e} \bar{V}(x, y) t
$$

with electron wavelength $\lambda_{e}$ and lattice potential $\bar{V}(x, y)$ averaged over the crystal thickness $t$ along the $z$-direction (parallel to the electron trajectory). In a small monoatomic crystal with atomic positions $\mathbf{R}_{m, n, o}=m \mathbf{a}_{1}+n \mathbf{a}_{2}+o \mathbf{a}_{3}$ (real-space basis $\left[\mathbf{a}_{1} \mathbf{a}_{2} \mathbf{a}_{3}\right]$ ), the total potential of the lattice is given by

$$
V(\mathbf{r})=V_{A}(\mathbf{r}) \otimes L(\mathbf{r})=V_{A}(\mathbf{r}) \otimes \sum_{m} \sum_{n} \sum_{o} \delta\left(\mathbf{r}-\mathbf{R}_{m, n, o}\right)
$$

with atomic potentials $V_{A}(\mathbf{r})$ in a periodic lattice $L(\mathbf{r})$.

In the far field, the scattered wave is proportional to the Fourier transform of the potential $\mathcal{F}[V(\mathbf{r})]=\mathcal{F}\left[V_{A}(\mathbf{r})\right] \cdot \mathcal{F}[L(\mathbf{r})][64]$. Not regarding the charge distribution of an individual atom $V_{A}(\mathbf{r})$, the lattice potential can be approximated by a three dimensional comb of Diracdelta functions in real-space (for a large number of atoms $m, n, o \rightarrow \infty$ ). Consequently, $\mathcal{F}[L(\mathbf{r})]$ yields intensity maxima in an equal manner on a three dimensional comb of Dirac-delta functions in reciprocal space (reciprocal basis $\left[\mathbf{b}_{1} \mathbf{b}_{2} \mathbf{b}_{3}\right]$ ). The scattering vector $\Delta \mathbf{k}$ is given by

$$
\Delta \mathbf{k}=\mathbf{k}-\mathbf{k}_{0}=h \mathbf{b}_{1}+k \mathbf{b}_{2}+l \mathbf{b}_{3},
$$


which for integer numbers $(h, k, l)$ is equivalent to the Laue-equation. In TEM literature, the basis of the reciprocal space is usually normalized to $\mathbf{a}_{i} \mathbf{b}_{j}=\delta_{i j}$ (omitting the $2 \pi$ prefactor) with Kronecker delta function $\delta_{i j}$. A set of lattice planes $(h k l)$ with spacing $d_{(h k l)}$ will lead to intense diffraction spots distanced by the absolute value of the scattering vector $|\Delta \mathbf{k}|=1 / d_{(h k l)}$, which corresponds to Bragg's law with scattering angle $\sin \theta \approx|\Delta \mathbf{k}| / k_{0}=$ $\lambda / 2 d_{(h k l)}$. Chapter 6 applies kinematic scattering theory to the analysis of local lattice deformations and will discuss further details like the influence of a finite crystal size and dynamic scattering features (Sec. 6.3), the local strain of a crystal (Sec. 6.6) and the dependence of scattering efficiency on the transient lattice temperature (Sec. 6.8.4).

\section{Strain analysis in TEM}

Over the last decades, the progress in transmission electron microscopy enabled quantitative far-field imaging of lattice potentials using parallel illumination or scanning-probe imaging [41], with down to single atom sensitivity [10, 65]. Nevertheless, quantitative two-dimensional characterization of subtle fields that are slowly varying with the atomic lattice remains an involving procedure. This includes the mapping of electromagnetic fields, e.g. at internal interfaces like charge depletion layers [66], as well as the quantitative reconstruction of local inhomogeneous strain fields, which is discussed in the following (cf. Fig. 2.4).

The local elastic deformation of a crystal lattice is described by the deformation gradient tensor $\mathbf{F}$, which in case of small deformations can be decomposed into the symmetric strain $\varepsilon$ and the anti-symmetric rotation tensor $\omega$ [43]

$$
\mathbf{F}(\mathbf{r})=\boldsymbol{\varepsilon}(\mathbf{r})+\omega(\mathbf{r})+\mathbf{I}_{3}
$$

with unit matrix $\mathbf{I}_{3}$. For more details on the action of $\mathbf{F}(\mathbf{r})$ on a crystal lattice, see Ref. [43] and Ch. 6. The following section compares different techniques for nanoscale strain analysis, with typical performance parameters given in Tab. 2.1.

Dark-field contrast of a strained crystal Collimated illumination of a crystalline samples, if rotated to closely match an efficient Bragg-scattering condition, will result in intense diffraction of the samples. A slight rotation of the lattice plane normal vector, sampling the diffraction rocking curve at a varying angle, yields a pronounced thin film bending contrast in the image (cf. Fig. 2.7) [36]. Notably, the quantitative interpretation of these bending 

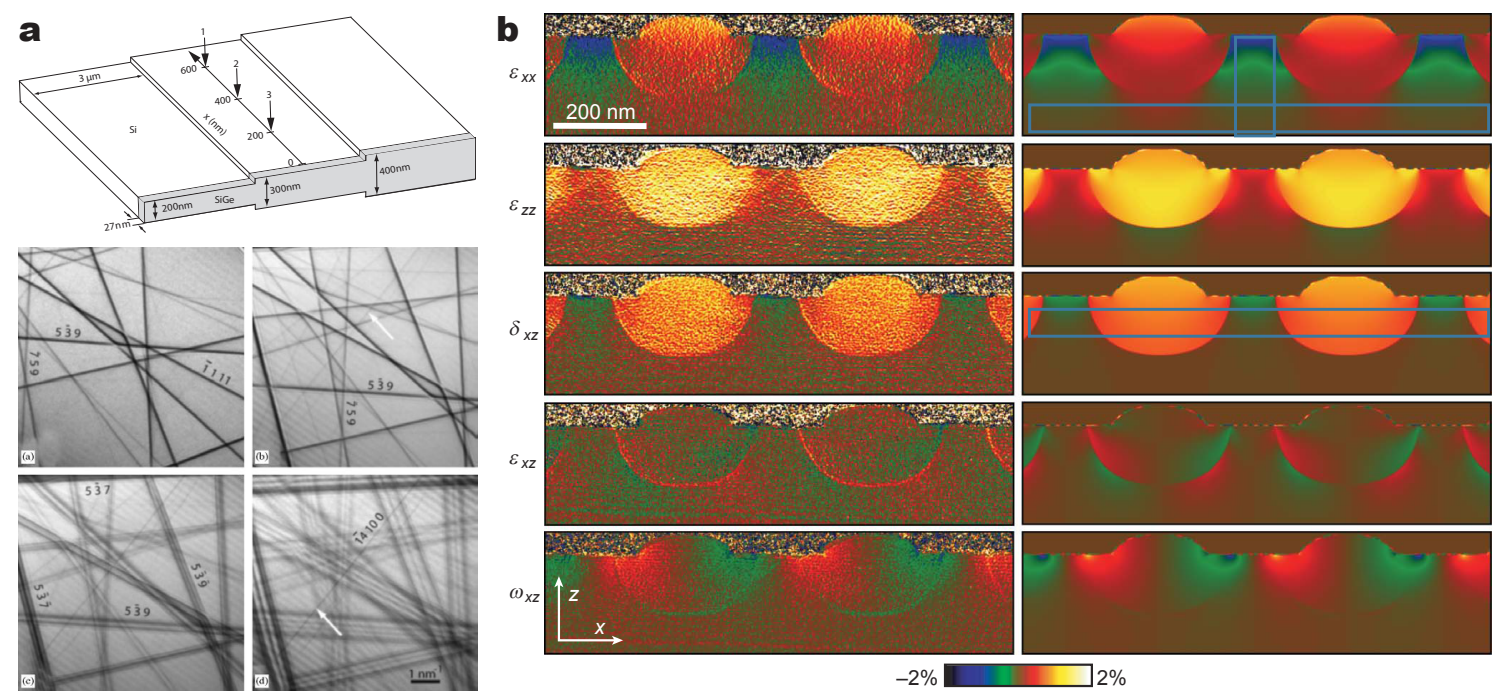

Figure 2.4: Nanoscale strain analysis in TEM. (a) Convergent beam electron diffraction (CBED) of a highly strained silicon substrate (capped by $\mathrm{Si}_{0.8} \mathrm{Ge}_{0.2}$, top panel), showing strain dependent Bragg-line splittings (bottom panel) [67]. (b) Experimentally measured (left) and numerically simulated (right) local lattice deformations of a strained-silicon transistor array obtained by dark-field electron holography [68]. (a) Reprinted from [67], Copyright 2006, with permission from Elsevier. (b) Adapted by permission from Springer Customer Service Centre GmbH: Springer Nature, Nature [68], () Macmillan Publishers Limited (2010).

contours is rather challenging, since they exhibit a directional sensitivity connected to the rocking curve of one specific Bragg-scattering condition and are overlapped to other phase-contrast features like thickness fringes [43].

Quantitative strain imaging A quantitative reconstruction of strain distributions is obtained by mapping the sample on the atomic level, either by high resolution TEM (HRTEM) [69] or scanning TEM (STEM) [70]. Alternatively, dark-field electron holography (DFEH) [68] directly measures the atomic displacement field by the interference of diffracted waves from strained and unstrained parts of a sample (cf. Fig. 2.4). Subsequently, the local strain fields can be decomposed from the obtained images by geometrical phase analysis (GPA) $[69,71]$. Both methods provide for high resolution imaging of strain fields in two-dimensions but have specific experimental requirements, e.g. thin samples, tilting to a zone-axis (HRTEM) or fully coherent sample illumination (DFEH). 
2.1 Transmission electron microscopy

CBED NBED HRTEM- DFEH STEM

\begin{tabular}{|c|c|c|c|c|}
\hline Sensitivity & $2 \times 10^{-4}$ & $1 \times 10^{-3}$ & $1 \times 10^{-3}$ & $2.5 \times 10^{-4}$ \\
\hline Accuracy & $2 \times 10^{-4}$ & $1-50 \times 10^{-3}$ & $1 \times 10^{-3}$ & \\
\hline Precision & & $0.9-10 \times 10^{-4}$ & $1-3 \times 10^{-3}$ & $2-20 \times 10^{-4}$ \\
\hline Spatial resolution $(\mathrm{nm})$ & $1-10$ & $2.5-10$ & $2-4$ & $4-6$ \\
\hline Field of view $(\mathrm{nm} \times \mathrm{nm})$ & \multicolumn{2}{|c|}{ map capable } & $150 \times 150$ & $500 \times 1500$ \\
\hline $\begin{array}{l}\text { Optimum specimen } \\
\text { thickness (nm) }\end{array}$ & $\sim 200$ & $\sim 50-100$ & $\sim 50-100$ & $\sim 100$ \\
\hline
\end{tabular}

Table 2.1: Comparison of quantitative strain analysis techniques. Reproduced from Ref. [43]

Diffractive strain mapping Nanoscale strain is directly correlated to a deformation of the local crystal unit cell. Therefore, local diffraction techniques combined with scanning of the electron probe are ideal tools for quantitative strain mapping [43]. A straightforward implementation is scanning nano beam electron diffraction (NBED) [72], which uses a $\mathrm{nm}$-sized collimated beam to record diffraction patterns.

In a more sophisticated approach, scanning convergent beam electron diffraction (CBED) makes use of a sharply focused electron probe. Due to the large angular content of the illuminating electron beam, multiple Bragg scattering conditions are studied simultaneously, which are visible as deficit and excess lines in the diffraction patterns (cf. Fig. 6.1). Adopting the experimentally observed line positions with calculations using a strained crystal allows for retrieving the local strain distribution [73]. Nano-beam diffraction poses strict requirements to the electron beam brightness and connects angular sensitivity with the achievable spatial resolution (cf. Eq. 3.12). In contrast, the strain sensitivity in CBED is decoupled from the illuminating beam's coherence, which only determines the minimum probing area for a given convergence angle [43].

Chapter 6 extends the versatile CBED methodology to the ultrafast timescale for the quantitative study of coherent strain dynamics with 28-nm spatial and 700-fs temporal resolution. 


\subsection{Elements of ultrafast electron diffraction and microscopy}

This thesis presents a novel instrumental approach in the field of UTEM by firstly incorporating high-coherence electron pulses from tip-shaped nanoscale photocathodes. The following section depicts the powerful experimental capabilities using state-of-theart large-area photoelectron emitters, but also highlights the opportunities of employing low-emittance electron pulses.

\section{Structural dynamics studied by ultrafast electron diffraction (UED)}

Electron diffraction, as first published in 1927 by Thomson and Reid [4], as well as Davisson and Germer [74], and awarded the 1937 Nobel Prize in Physics, quickly became a well-established technique for the structural analysis of condensed matter [64]. Different experimental implementations involve surface-sensitive back-scattering of electrons in low-energy electron diffraction (LEED), reflection-high-energy electron diffraction (RHEED) or electron backscatter diffraction (EBSD), while thicker samples are analyzed by high-energy electrons in transmission electron diffraction (TED). With the availability of ultrashort electron pulses, these techniques were very successfully transferred to the ultrafast timescale, combining a femtosecond temporal with a sub-pm resolution in k-space.

Transmission electron diffraction Figure 2.5 displays typical (a,b) parallelillumination and (c) convergent beam diffraction data of graphite crystals in transmission. Individual Bragg diffraction conditions are observed with their position and intensity recorded as a function of pump-probe time delay. On short time-scales the reduction of scattered intensity is associated with a rise of the lattice temperature and atomic mean-square displacements (Fig. 2.5a,b). This phonon-mode specific Debye-Waller behavior gives information about the complex dissipation cascade of electronic excitation to thermal phonons. Following on the picosecond to nanosecond timescale (Fig. 2.5c), the synchronous excitation of phonons induces coherent lattice deformations related to the thin-film sample geometry, apparent by periodic shifts of Bragg spots. Both effects will be discussed in more details in Ch. 6, addressing the capabilities of convergent beam electron diffraction (CBED) using nanoscale probing areas. 

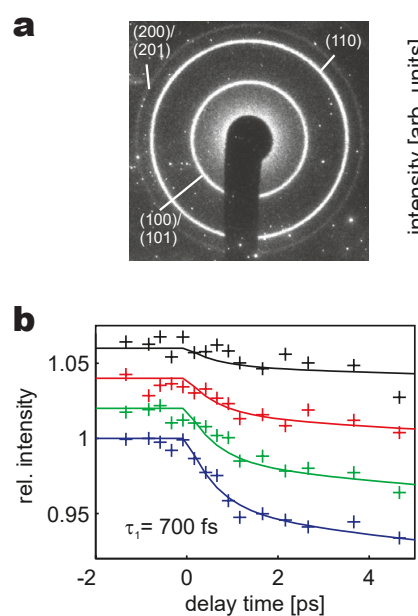
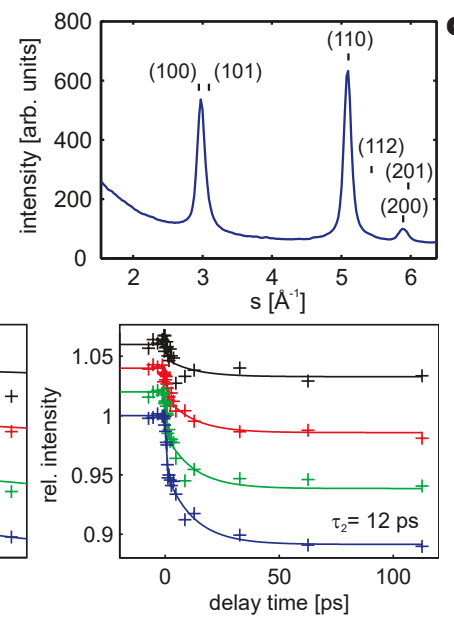

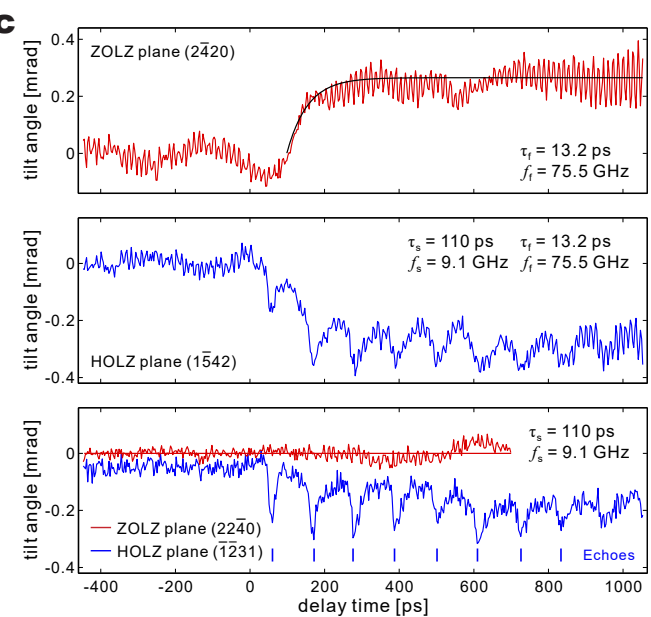

Figure 2.5: Ultrafast electron diffraction (UED) in transmission. (a) Powder diffraction pattern and radially averaged intensity profile of thin film graphite (1 to 3-nm thickness). (b) Ultrafast drop in diffracted intensity of the (110) diffraction peak (from top: $18,35,53$ and $70 \mu \mathrm{J} / \mathrm{cm}^{2}$ excitation fluence) displays two characteristic time constants involved in the thermalization of the initial nonequilibrated phonon system [75]. (c) Convergent beam diffraction with $\mu \mathrm{m}$-scale electron spot size of single crystalline graphite (220-nm thickness) reveals the excitation of multiple membrane deformation modes observed in the oscillating position of specific Bragg reflections [76]. $(a, b)$ Copyright The Authors [75] used in accordance with the Creative Commons Attribution (CC BY) license. (c) Copyright 2014 National Academy of Sciences. 

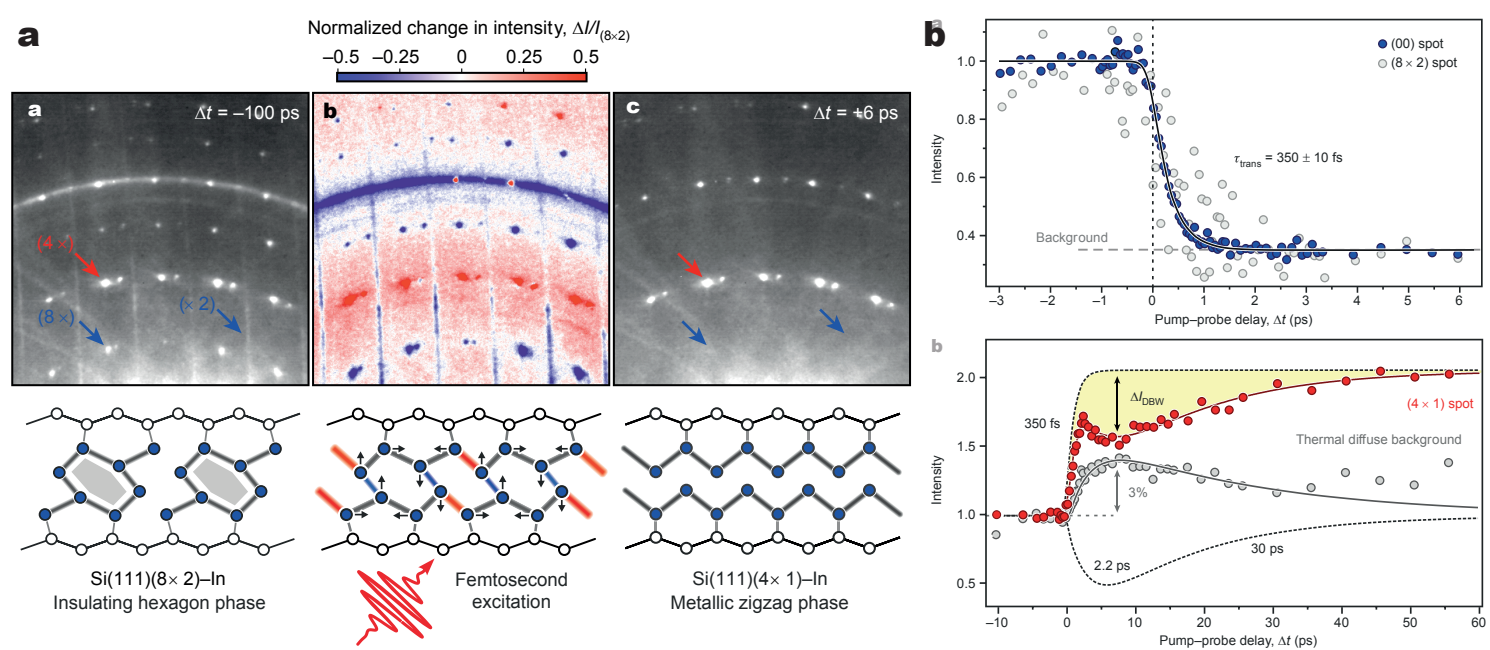

Figure 2.6: Ultrafast electron diffraction in reflection. (a) Time-resolved RHEED and surface structure illustration of the In on $\mathrm{Si}(111)$ surface reconstruction under ultrafast phase transition. (b) Time evolution of the diffracted intensity related to atomic lattice Bragg-reflections ((00)lattice spots), charge density wave ( $8 \times 2$ and $4 \times 1$ spots) and thermal diffuse background. For more details, see Ref. [78]. Adapted by permission from Springer Customer Service Centre GmbH: Springer Nature, Nature [78], (C) 2017 Macmillan Publishers Limited, part of Springer Nature (2017).

Electron diffraction in reflection The superior surface sensitivity of low-energy [77] or grazing incidence electron diffraction [78] allows for the study of structural dynamics of a few crystal layers at the surface. Figure 2.6 shows an example of ultrafast RHEED probing a non-thermally excited structural phase-transition of the $\mathrm{In}-(8 \times 2)$ on $\mathrm{Si}(111)$ surface reconstruction [78]. Despite probing the surface under grazing incidence with a relatively large electron spot, a temporal resolution of down to $330 \mathrm{fs}$ is achieved by appropriate tilting of the pump laser pulse front and reducing the probe pulse charge to below 200 electrons per pulse. The combination of surface sensitivity and high resolution in reciprocal space enables a unique access to structural transitions, like the ultrafast melting of charge density waves (CDW) at surfaces and the decoupling from bulk material dynamics (cf. Fig. 2.6b).

\section{Real-space probing of structural dynamics}

Conventional electron microscopes routinely deliver imaging and local probing capability of condensed matter down to the atomic level. The recent availability of femtosecond pulsed laser systems for the generation of ultrashort electron pulses and intrinsically 

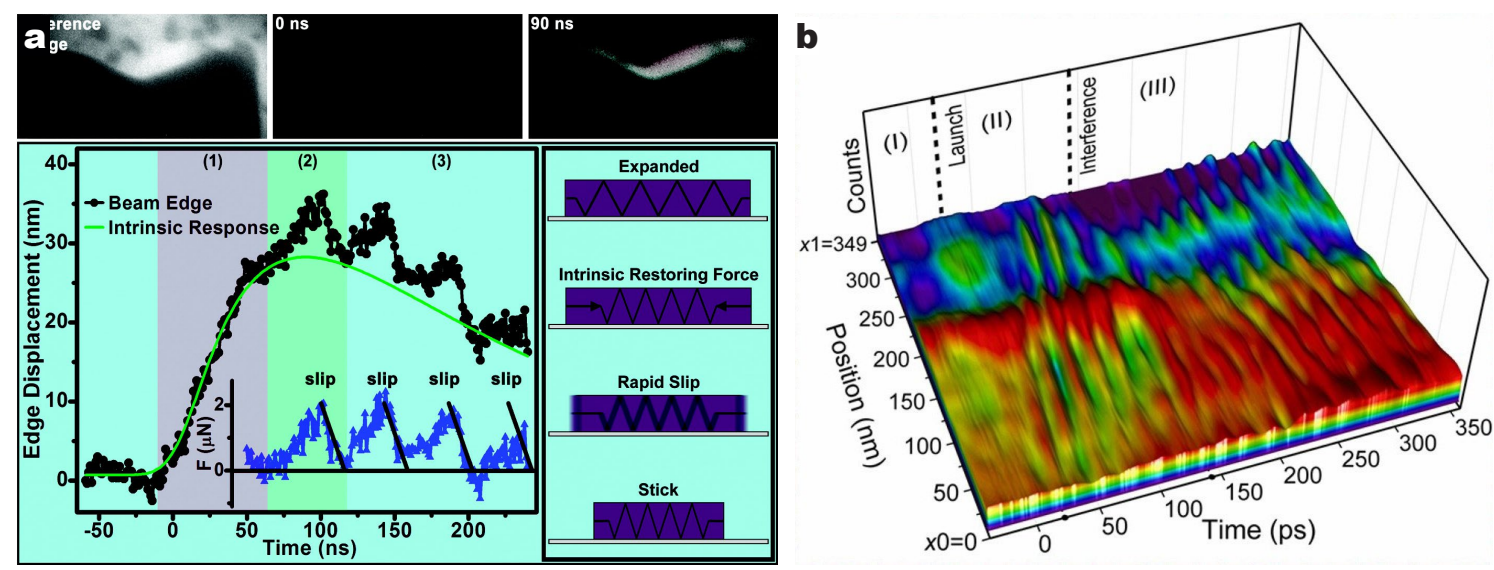

Figure 2.7: Imaging strain-stress dynamics in UTEM. (a) Slip-stick dynamics of a single crystal $\mathrm{Cu}(\mathrm{TCNQ})$ nanoparticle following laser induced thermal expansion. Reference and difference images of crystallite edge (stroboscopic illumination, top) with extracted time dependent edge displacement (bottom) [81]. (b) Ultrafast time-dependent bright-field imaging of $\mathrm{MoS}_{2}$ single crystal. Image contours at fixed spatial position as function of time delay show the launching, propagation and interference of acoustic wave-trains [85]. (a) Reprinted with permission from [81]. Copyright 2010 American Chemical Society. (b) Reprinted with permission from [85]. Copyright 2017 American Chemical Society.

synchronized sample excitation enabled novel applications in ultrafast electron imaging.

Studying nanophononic and nanomechanic systems A field of applications ideally suited for ultrafast TEM is the study of the reversible mechanical sample response following optical excitation, which is closely related to the frequency domain studies in the field of nanophononics [79]. Typical sound velocities in condensed matter around $1-20 \mathrm{~nm} / \mathrm{ps}$ result in spatio-temporal dynamics well within the reach of ultrafast TEM.

The first type of experiments relies on real-space imaging, e.g. of nanocrystals or cantilevers showing oscillatory movement [80] or slip-stick like behavior related to friction (cf. Fig. 2.7a) [81]. Furthermore, the dark- or bright-field contrast derived from scattering at the deformed crystal lattice yields a qualitative image of ultrafast local lattice dynamics $[82,83]$, recently applied for studying phonon dynamics in thin membranes or wedge shaped single crystals (cf. Fig. 2.7b) [84-87].

In a second approach, a focused electron beam diffractively probes the local structural deformation of a crystalline thin film. In an initial experiment, Yurtsever et al. retrieved the quantitative coherent strain dynamics at a single probing position of a wedged silicon sample by analyzing the time dependent shift of Kikuchi-lines (cf. Fig. 2.8) [88, 89]. 

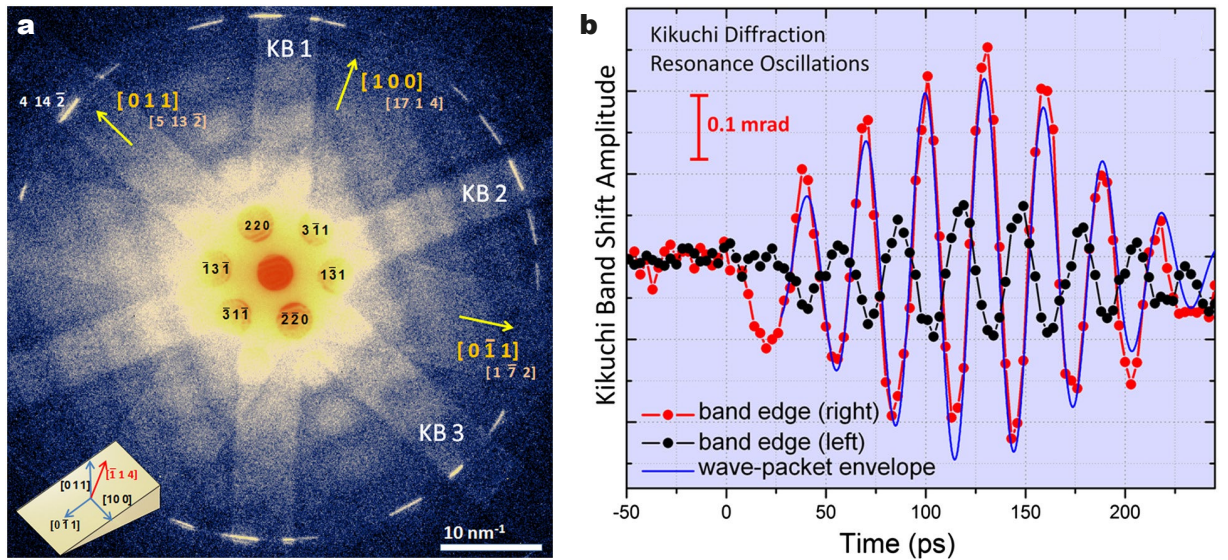

Figure 2.8: Ultrafast local diffractive probing in UTEM. (a) Convergent-beam Kikuchi diffraction pattern of a wedged silicon single crystal (inset). (b) Time-delay dependent resonant oscillation of the KB 1 Kikuchi band position, sampling a laterally propagating elastic shear wave. For details see Ref. [88]. Reproduced with permission from [88]. Copyright 2011 National Academy of Sciences.

Extending this concept, Ch. 6 presents the implementation of ultrafast convergent beam electron diffraction (U-CBED), making use of the full quantitative strain retrieval capability of CBED combined with a nanometer-precision lateral scanning of the focused electron beam. The combined 700-fs temporal and 28-nm spatial resolution resolves the coherent laser-excited lattice dynamics at the edge of a thin graphite membrane.

\section{Inelastic electron light scattering (IELS) and photon induced near-field electron microscopy (PINEM)}

Historically, inelastic scattering processes in TEM are associated with a spontaneous energy transfer from the electron beam to a sample, as analyzed in electron energy loss spectroscopy (EELS) [92] or by light emission in cathodoluminescence (CL) [93]. Spontaneous energy gain scattering requires interaction with densely populated excited states, e.g. hot phonon distributions [94], and typically necessitates an excellent spectral resolution to resolve low-energy excitations [95]. An equivalent scheme, termed electron energy-gain spectroscopy (EEGS), was proposed for studying plasmon resonances of optically excited nanostructures [93, 96, 97].

This stimulated gain scattering process was first observed in UTEM by Barwick et al. [90], probing the near-field of optically excited nanostructures with synchronized ultrashort electron pulses (cf. Fig. 2.9). Here, high-energy electrons (electron kinetic 

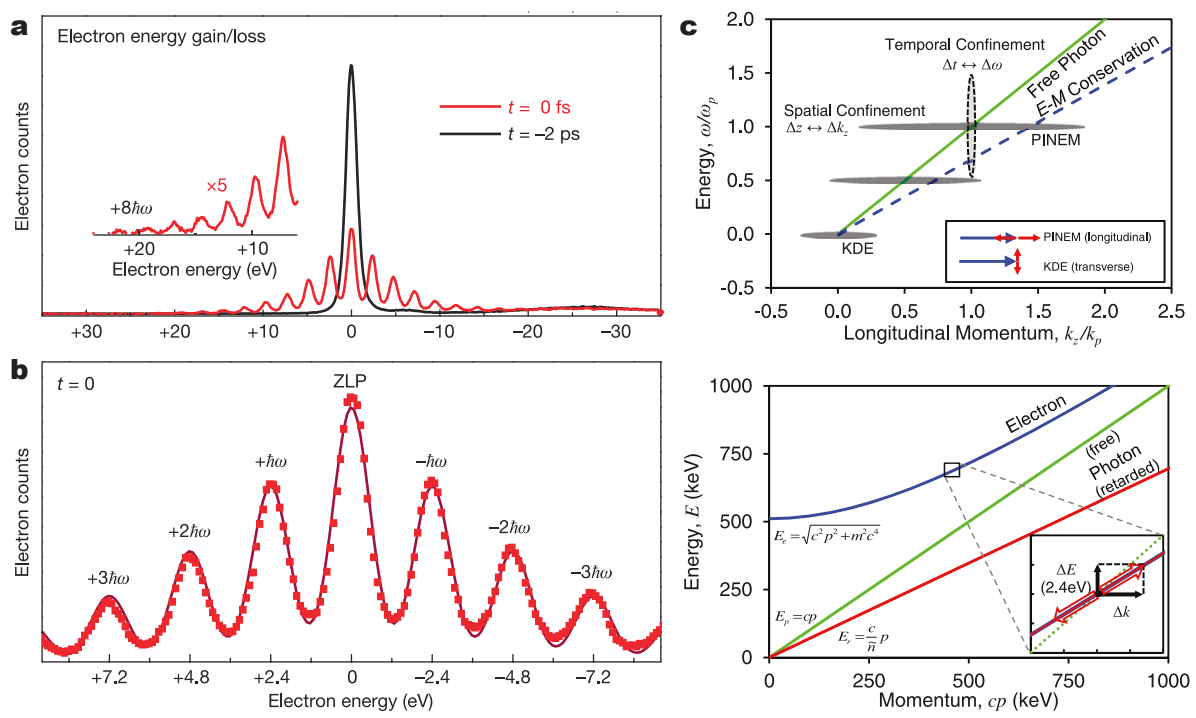

Figure 2.9: Inelastic electron light scattering (IELS) in optical near-fields. (a) Electron energy spectra (centered at $200-\mathrm{keV}$ electron kinetic energy) of carbon nanotubes irradiated with an intense fs-laser pulse at two different delay times. The initial zero-loss peak (black curve) evolves into a comb of spectral sidebands displaying (b) multiple quanta $\pm N \hbar \omega$ of photon absorption/emission (red curve) [90]. (c) The (relativistic) dispersion relations of electrons and photons are not matched in free space (bottom), preventing single particle scattering. Energymomentum conservation in electron-photon scattering is enabled by the broadened momentum distribution of optical near-fields [91]. (a,b) Reprinted by permission from Springer Customer Service Centre GmbH: Springer Nature, Nature, [90], () Macmillan Publishers Limited (2009). (c) Copyright The Authors [91] used in accordance with the Creative Commons Attribution (CC BY) license.

energy of $200 \mathrm{keV}$ ) passing an intense optical near-field are subject to a multi-photon emission/absorption process, which results in the formation of spectral sidebands in their kinetic electron-energy distribution (cf. Fig. 2.9a,b and Fig. 5.1). Notably, in free space, this electron-photon interaction is forbidden due to a mismatch of their respective dispersion relations $^{1}$ (cf. Fig. 2.9c). For a more detailed theoretical description, see Ch. 5 and Refs. $[91,98]$.

The spatially confined near-field of an optically excited nanostructure provides a broadened momentum distribution, enabling inelastic electron light scattering (IELS) (cf. Fig. 2.9c). The image contrast obtained by energy-filtering of the gain-scattered fraction of electrons was used to image non-resonant [90] and resonant [99] optical near-

\footnotetext{
${ }^{1}$ photon dispersion relation $E_{\mathrm{ph}}=c|\mathbf{p}|$ with photon momentum $\mathbf{p}$. Compare to that of free-electrons according to Eq. 3.17 .
} 

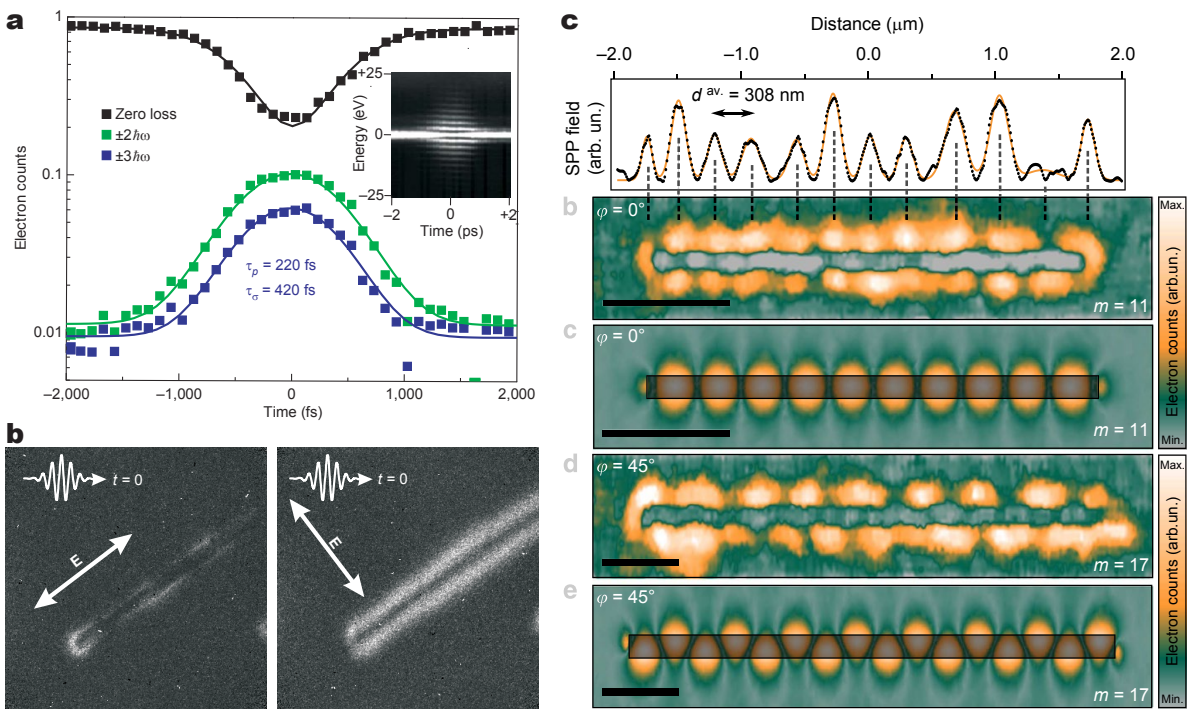

Figure 2.10: Photon-induced near-field electron microscopy (PINEM). (a) Zero-loss peak (ZLP) and population of selected sidebands (log-scale) as function of relative pump-probe time delay. Electron-photon cross-correlation yields a measure for probing electron pulse durations [90]. $(b, c)$ PINEM: Energy filtered TEM images (using gain scattered electrons). (b) Near-field of an individual carbon nanotube imaged for parallel and perpendicular polarization of the exciting light field [90]. (c) Laser induced surface plasmon polariton (SPP) standing wave pattern at isolated $\mathrm{Ag}$ nanowires. Note the excitation of an asymmetric SPP mode $(m=17)$ by using $45^{\circ}$ azimuthal incidence polarization [99]. (a,b) Reprinted by permission from Springer Customer Service Centre GmbH: Springer Nature, Nature, [90], () Macmillan Publishers Limited (2009). (c) Copyright The Authors [99] used in accordance with the Creative Commons Attribution (CC BY) license.

fields in a method termed photon-induced near-field electron microscopy (PINEM) (cf. Fig. 2.10b,c). Additionally, due to the short transition times of high-energy electrons passing the optical near-field, electron-photon cross-correlation in IELS allows for characterization of the longitudinal electron phase space distribution (cf. Fig. 2.10a) [90, $100]$.

The inherent coherence of this process was theoretically predicted [91, 98], but the associated spectral features were not observed in early experiments due to spatial and temporal averaging. Chapter 5 presents the first experimental demonstration of the quantum coherent nature of IELS, by applying low-emittance ultrashort electron pulses and quasi continuous wave $(\mathrm{cw})$ optical excitation. The precise tailoring of the electron wave function in longitudinal direction opens up new avenues for the coherent optical control of free-electron beams (cf. Sec. 7.2). 


\section{Chapter 3}

\section{Properties and generation of ultrashort electron pulses}

The usefulness of radiation or particle beams for practical experiments crucially depends on the availability of suitable sources and their emission properties. Following emission, processes like free-space propagation, optical elements or interaction with matter may further alter beam properties in a reversible or irreversible way. In optics, the advent of coherent light sources and the capability to manufacture highly elaborate optical systems enabled a multitude of easy-to-access applications. Especially the bosonic nature, lack of charge and dispersion-free vacuum propagation of photons allow for the generation of ultrashort laser pulses of excellent usability with pulse durations down to the attosecond time scale. In contrast, creating high quality electron beams poses multiple challenges. The strong particle-particle interactions due to their fermionic character and finite charge may induce reversible and irreversible pulse degradation. Furthermore, the dispersive propagation in free-space requires strategies to maintain short pulse durations.

All of that necessitates a comprehensive description of pulsed electron beams, their generation and evolution upon propagation, which is addressed in the following.

\subsection{Phase space description of electron beams}

Conventionally, a geometric particle beam refers to the concept of a stream of isolated particles having a common direction of propagation and being laterally confined [101]. Within the description of an electron beam, a natural orthogonal coordinate decoupling parallel ( $z$-axis, longitudinal) and perpendicular ( $x$-, $y$-axes, transverse) to the main direction of propagation can be applied, accounting for an order of magnitude larger longitudinal electron momentum (fulfilling paraxial approximation) [101]. By this separation, the 
longitudinal beam properties are directly connected the electron beam's energy distribution and evolving temporal profile (at a fixed $z$-position).

\section{Electron pulses in classical phase space}

In classical mechanics, the state of a single particle in three-dimensional space is given by a set of canonical position $q_{i}$ and momentum $p_{i}$ coordinates $(i=x, y, z)$, with its propagation described by a trajectory in six-dimensional phase space. Extending this concept, the temporal evolution of an ensemble of electrons may be described by a time-dependent local particle density $n\left(q_{i}, p_{i}, t\right)$. For such a phase space distribution, Liouville's theorem states that the volume occupied by a given number of non-interacting particles remains constant $[101,102]$. This is valid in good approximation if single particle-particle interactions can be neglected and electromagnetic forces induced by the electrons are well described by an average scalar potential [101]. Integrating the particle density $n\left(q_{i}, p_{i}\right)$ in phase space yields the total number of electrons

$$
N_{e}=\iiint n\left(q_{i}, p_{i}\right) \mathrm{d} q_{i} \mathrm{~d} p_{i}
$$

In general, a propagating electron (or ensemble of many thereof) may be described as a classical density function $\rho$ in six dimensional phase space with canonical coordinates $q_{i}$ and $p_{i}(i=x, y, z)$. The total charge

$$
Q=N_{e} \cdot e=\iiint \rho\left(q_{i}, p_{i}\right) \mathrm{d} q_{i} \mathrm{~d} p_{i},
$$

of such an electron bunch is $N_{e}$ times the elementary charge $e$ and obtained by integrating the charge distribution $\rho\left(q_{i}, p_{i}\right)$ over phase space. To characterize the occupied phase space volume of such an electron bunch, a useful quantity is the normalized rms (root-meansquare) beam emittance, which is independent of the kind of particle, projecting the $6 \mathrm{D}$ phase space distribution onto the three orthogonal subspaces $\left(q_{i} p_{i}\right)$ with $(i=x, y, z)[101]$

$$
\varepsilon_{\mathrm{n}, \mathrm{rms}, i}=\frac{1}{m_{e} c} \sqrt{\left\langle q_{i}^{2}\right\rangle\left\langle p_{i}^{2}\right\rangle-\left\langle q_{i} p_{i}^{\prime}\right\rangle^{2}}
$$

with $\sqrt{\left\langle q_{i}^{2}\right\rangle}=\sigma_{q_{i}}$ and $\sqrt{\left\langle p_{i}{ }^{2}\right\rangle}=\sigma_{p_{i}}$ giving the standard deviations of position and momentum contained in the beam, while the cross-term $\left\langle q_{i} p_{i}^{\prime}\right\rangle^{2}$ accounts for linear phase space distortions (e.g. elliptical non-parallel to $p_{i}$ or $q_{i}$ ). At a beam waist, e.g. in a spatial or 


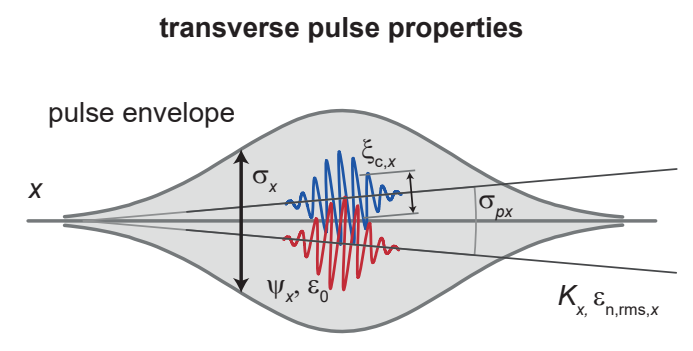

6D properties: $m_{\mathrm{e}}, N_{\mathrm{e}}, \mathrm{Q}, B^{6 D}, \Omega^{6 \mathrm{D}}$ longitudinal pulse properties

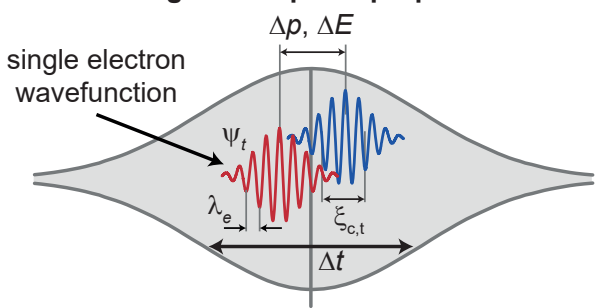

$t, v_{0}, p_{0}, E_{0}$

Figure 3.1: Properties of single-electron pulses. The concept of a pulsed electron beam enables a decoupling of the transverse and longitudinal phase space. A quantitative description is given by the ensemble properties of multiple low-charge electron pulses generated at a high repetition rate.

temporal focus of a beam, the cross-term vanishes, reducing Eq. 3.3 to

$$
\varepsilon_{\mathrm{n}, \mathrm{rms}, i}=\frac{1}{m_{e} c} \sigma_{q_{i}} \sigma_{p_{i}} .
$$

This normalization allows for defining the brightness of an electron bunch in terms of its total charge divided by the occupied phase space volume $\bar{B}_{6 \mathrm{D}}=Q / V_{6}$, or explicitly, by integrating the differential brightness

$$
\mathrm{d} B^{6 \mathrm{D}}=\left(m_{e} c\right)^{3} \frac{\mathrm{d}^{6} \rho}{\mathrm{d} x \mathrm{~d} y \mathrm{~d} z \mathrm{~d} p_{x} \mathrm{~d} p_{y} \mathrm{~d} p_{z}},
$$

over the entire phase space $\left((x, y, z)=\left(q_{x}, q_{y}, q_{z}\right)\right.$ for clarity). In case of Gaussian shaped distributions of $q_{i}$ and $p_{i}$, the peak brightness in the center of the bunch is given by [103]

$$
B_{\mathrm{np}}^{6 \mathrm{D}}=\frac{1}{8 \pi^{3}} \frac{Q}{\varepsilon_{\mathrm{n}, \mathrm{rms}, x} \varepsilon_{\mathrm{n}, \mathrm{rms}, y} \varepsilon_{\mathrm{n}, \mathrm{rms}, z}} .
$$

In good approximation, the $6 \mathrm{D}$ phase space decouples into a transverse $\left(x, y, p_{x}, p_{y}\right)$ and longitudinal $\left(z, p_{z}\right)$ subspace, assuming near-monochromatic (low initial longitudinal momentum spread), non-interacting (free of space charge and scattering) and high energy electron beams of low divergence $\left(p_{z} \approx p_{0}\right)$ [101]. All of that is fulfilled for single-electron pulses accelerated linearly by a high static potential, as later applied in this thesis (cf. Ch. 4) and allows for a separate treatment of the longitudinal and transverse beam properties, as illustrated in Fig. 3.1. 


\section{Quantum phase space: The Wigner distribution}

To account for their simultaneous wave and particle characters, real particles must be described as complex quantum mechanical wave packets $\psi\left(p_{i}, t\right) \stackrel{\mathcal{F}}{\leftrightarrow} \psi\left(q_{i}, t\right)$ (connected by the Fourier transform $\mathcal{F}$ ). Here, the particles classical canonical coordinates are substituted by the expectation values of the position $q_{i} \rightarrow\left\langle q_{i}\right\rangle$ and momentum operator $p_{i} \rightarrow\left\langle p_{i}\right\rangle$. Additionally, such a matter wave description provides for the particle's corresponding de Broglie wavelength $\lambda_{e}=h /\left|p_{0}\right|$ with Planck constant $h$ and electron momentum in direction of propagation $p_{0}$. In analogy to the classical phase space density, the Wigner distribution of a pure quantum state is defined by [104]

$$
W_{i}\left(q_{i}, p_{i}\right)=\frac{1}{\pi \hbar} \int_{+\infty}^{-\infty} \psi^{*}\left(q_{i}+q_{i}^{\prime}\right) \psi\left(q_{i}-q_{i}^{\prime}\right) e^{-2 i p_{i} q_{i}^{\prime} / \hbar} \mathrm{d} q_{i}^{\prime}
$$

for the conjugate variables $q_{i}$ and $p_{i}$, as will be applied for studying the temporal evolution of the longitudinal electron wave function in $\mathrm{Ch}$. 5. Note that the generalized Wigner distribution in case of mixed quantum states is an established tool for describing the transverse ensemble properties of electron beams in electron optical systems, including lenses, aberrations and holography, and is applied for the description of coherent and incoherent sample interactions [105-107].

Heisenberg's uncertainty principle applies, stating a lower bound for the consecutive measurement of position and linear momentum $\sigma_{q_{i}} \cdot \sigma_{p_{i}} \geq \hbar / 2$, here represented by the standard deviations of the respective operators. In consequence, the single electron wave function always occupies a finite volume in phase space, which results in a lower limit of the normalized rms emittance

$$
\varepsilon_{\mathrm{n}, \mathrm{rms}, i}=\frac{1}{m_{e} c} \sigma_{q_{i}} \sigma_{p_{i}} \geq \frac{\hbar}{2 m_{e} c}=\varepsilon_{0}=0.26 \cdot 10^{-12} \mathrm{~m}
$$

in all Cartesian projections (identity applies for a Gaussian shaped wave packet). Interestingly, one interpretation of Heisenberg's principle is the quantization of the classical phase space, which yields the volume $\mathrm{d} V_{0}=\left(m_{e} c \varepsilon_{0}\right)^{3}$ that a single electron microstate occupies. Hereby, the microcanonical degeneracy of an ensemble of electron pulses in classical phase can be defined by [108]

$$
\Omega^{6 \mathrm{D}}=\frac{\mathrm{d} V_{0} \cdot N_{e}}{V_{6}}=\frac{\varepsilon_{0}^{3}}{\varepsilon_{\mathrm{n}, \mathrm{rms}, x} \varepsilon_{\mathrm{n}, \mathrm{rms}, y} \varepsilon_{\mathrm{n}, \mathrm{rms}, z}} .
$$


Notably, Pauli's exclusion principle allows no more than two electron spin states occupying a phase space element $\mathrm{d} V_{0}$, therefore limiting the degeneracy to $\Omega^{6 \mathrm{D}} \leq 2$. Combining both principles yields a theoretical maximum for the brightness of an electron beam,

$$
B_{\mathrm{np}}^{6 \mathrm{D}}=\frac{2 e}{\mathrm{~d} V_{0}}=2 e \cdot\left(\frac{2 m_{e} c}{\hbar}\right)^{3}=4.45 \cdot 10^{19} \frac{\mathrm{C}}{\mathrm{m}^{3}} .
$$

For most applications, an electron beam is sufficiently well described by the classical density function $\rho$ if the lower limit of the emittance $\varepsilon_{0}$ is included, e.g. yielding the well-known diffraction limit in the transverse beam direction.

\section{Transverse beam properties}

In a microscope or accelerator, the most easily accessible transverse beam properties are the beam diameter and opening angle of the focused particle beam. Therefore, in analogy to geometrical optics for paraxial approximation $\left(p_{x, y} \ll p_{z}\right)$, the transverse electron momenta $p_{x}, p_{y}$ can be replaced by the slopes $x^{\prime}, y^{\prime}$ of the electron beam [101]

$$
x^{\prime}=\frac{p_{x}}{P_{z}} \quad y^{\prime}=\frac{p_{y}}{P_{z}} .
$$

The geometrical normalized rms emittance is given by

$$
\varepsilon_{\mathrm{n}, \mathrm{rms}, x}=\beta \gamma \sqrt{\left\langle x^{2}\right\rangle\left\langle x^{\prime 2}\right\rangle-\left\langle x x^{\prime}\right\rangle^{2}} \quad \varepsilon_{\mathrm{n}, \mathrm{rms}, y}=\beta \gamma \sqrt{\left\langle y^{2}\right\rangle\left\langle y^{\prime 2}\right\rangle-\left\langle y y^{\prime}\right\rangle^{2}},
$$

for longitudinal electron momentum $p_{z} \approx p_{0}=\beta \gamma m_{e} c$, relativistic Lorentz factor $\gamma$ and $\beta=v_{e} / c$ with $v_{e}$ and $c$ being the electron and light velocities in vacuum, respectively. In the waist of a Gaussian beam (which corresponds to an axis-aligned elliptical $\left(x, p_{x}\right)$ distribution), the divergence terms become zero, simplifying Eq. 3.12 by substitution with the standard deviations of the beam diameter $\sigma_{x}$ and angular spread $\sigma_{x^{\prime}}$

$$
\varepsilon_{\mathrm{n}, \mathrm{rms}, x}=\beta \gamma \cdot \sigma_{x} \sigma_{x^{\prime}} \quad \varepsilon_{\mathrm{n}, \mathrm{rms}, y}=\beta \gamma \cdot \sigma_{y} \sigma_{y^{\prime}} .
$$

Notably, Liouville's theorem also applies in good approximation to the transverse trace space, allowing for measuring the emittance of an electron source at a later beam waist in an optical system - if the beam was not cut by an aperture. Applying Eq. 3.13 only yields an upper bound for the emittance, since phase space distortions (like lens aberrations) will 
lead to a non-vanishing divergence term.

While the 6D brightness defined by Eq. 3.6 gives the peak charge density of a Gaussian shaped bunch, an often more practical quantity for characterizing the usability of an electron beam is the $4 \mathrm{D}$ brightness [103]

$$
B_{\mathrm{np}}=\frac{1}{4 \pi^{2}} \frac{I}{\varepsilon_{\mathrm{n}, \mathrm{rms}, x} \cdot \varepsilon_{\mathrm{n}, \mathrm{rms}, y}},
$$

which is a projection into the transverse 4D subspace. Notably, two beams of different longitudinal momentum spread (corresponding to broader energy distribution) but same current and transverse geometrical properties have the same 4D brightness.

A further important property of an electron beam is the transverse degree of coherence, corresponding to the capability of self-interference. For classical electron sources, an electron wavefunction only interferes with itself due to an initial random phase upon generation. Therefore, to observe perfect ensemble interference, all electrons must occupy only one (spin independent) state in transverse phase space, which enables the definition of the transverse degree of coherence by [109]

$$
K_{x}=\frac{\varepsilon_{0}}{\varepsilon_{\mathrm{n}, \mathrm{rms}, x}} \leq 1
$$

The lateral coherence length $\xi_{\mathrm{c}, x}$ is given by the diameter of a single electron wavefunction $\psi$ (twice the standard deviation of the position operator, $2 \sigma_{\psi, x}$ ) [110]. Consequently, Heisenberg's uncertainty principle relates the transverse momentum spread $\sigma_{p_{x}}$ of a beam with its coherence length and substitution with Eq. 3.4 yields [111]

$$
\xi_{\mathrm{c}, x}=\hbar / \sigma_{p_{x}}, \quad \xi_{\mathrm{c}, x}=\frac{\hbar}{m_{e} c} \cdot \frac{\sigma_{x}}{\varepsilon_{\mathrm{n}, \mathrm{rms}, x}} .
$$

Alternatively, the transverse coherence length of a wave can be derived in a more stringent way by considering the Van Cittert-Zernike theorem and calculating the coherence transfer functions [109, 112].

\section{Longitudinal beam properties}

In longitudinal phase space, an electron beam is most conveniently described in terms of its temporal structure and kinetic energy distribution. The spatial (laboratory-fixed) 
coordinate $z$ in beam direction is replaced with the propagation time $t=z / v_{e}$ by considering the relativistic electron velocity $v_{e}$. The kinetic energy $E_{0}$ of the electrons after acceleration in a static potential $U_{0}$ is connected to the longitudinal momentum component $p_{0} \approx p_{z}$ by the relativistic dispersion relation

$$
E_{0}=U_{0} \cdot e=\sqrt{p_{0}^{2} c^{2}+m_{e}^{2} c^{4}}-m_{e} c^{2}
$$

with slope $\mathrm{d} E / \mathrm{d} p_{0}=v_{e}$, yielding $p_{0}=\frac{1}{c} \sqrt{E_{0}^{2}+2 E_{0} m_{e} c^{2}}$ solved for the momentum. Substituting $\mathrm{d} p_{0}=\frac{1}{v_{e}} \mathrm{~d} E$ and $\mathrm{d} z=v_{e} \mathrm{~d} t$ in Eq. 3.4 gives an alternative definition for the longitudinal normalized rms beam emittance

$$
\varepsilon_{\mathrm{n}, \mathrm{rms}, z}=\frac{1}{m_{e} c} \sigma_{t} \sigma_{E} \geq \varepsilon_{0}=\frac{\hbar}{2 m_{e} c}
$$

using the rms bunch duration $\sigma_{t}$ and energy spread $\sigma_{E}$. In analogy to Eq. 3.16, the temporal coherence length of an electron beam is given by

$$
\xi_{\mathrm{c}, t}=\hbar v_{e} / \sigma_{E}, \quad \xi_{\mathrm{c}, t}=\frac{\hbar}{m_{e} c} \cdot \frac{\sigma_{x}}{\varepsilon_{\mathrm{n}, \mathrm{rms}, x}}
$$

This brief introduction to the theoretical description of electron pulses in phase space will be essential for Ch. 4, characterizing the Göttingen UTEM instrument, and for Ch. 5 using the Wigner function to study the temporal phase space evolution of single-electron pulses.

\subsection{Time evolution of phase space distributions}

Like all particles of finite rest mass, electrons are subject to dispersive propagation in vacuum with the particle velocity depending on its kinetic energy. In a co-moving spatial coordinate frame, time evolution causes a shearing of the electron phase space distribution (cf. Fig. 3.2), i.e. electrons at higher momenta are propagating faster. In the transverse beam direction, such a description is routinely used for free-space drift and optical elements with laterally inhomogeneous fields like magnetic and electrostatic lenses [39, 105, 113].

In the longitudinal beam direction, propagation (at non-relativistic velocities) causes an initially Gaussian shaped phase space distribution to evolve a linear chirp, corresponding to a linear increase of the energy with spatial coordinate $z$ along the beam axis (at a fixed time). Therefore, fast electrons arrive before slower ones. This effect is discussed in 


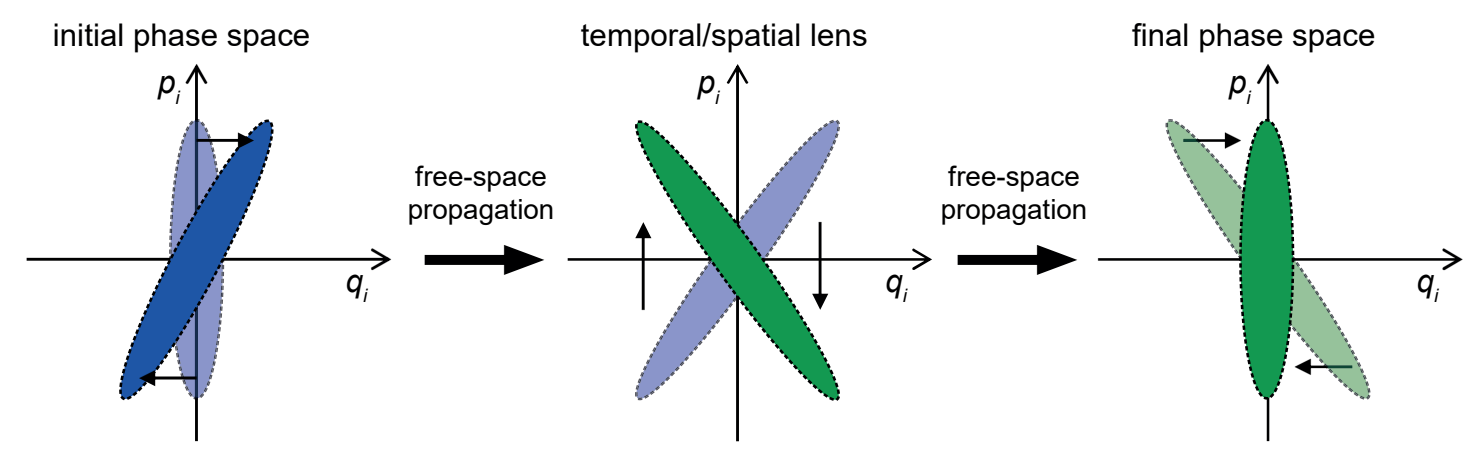

Figure 3.2: Temporal evolution of phase space distributions. Upon propagation, the initial phase space distribution $\rho\left(q_{i}, p_{i}\right)$ is sheared along the momentum axis $p_{i}$, evolving a linear chirp. A transverse or longitudinal inversion of $p_{i}$ (relative to $\left\langle p_{i}\right\rangle$ ) constitutes a spatial or temporal lens and results in a later re-focusing in phase space.

section 4.3.3 and leads to an increasing pulse duration with time. The same concepts apply for the temporal evolution of the quantum phase space, which is discussed in Sec. 5.4.5.

Nevertheless, if the longitudinal phase space volume is conserved, such temporal distributions can be re-compressed by time-varying optical elements, as applied in resonant cavities operating in the radio frequency regime (cf. Fig. 3.3). For example, inverting the longitudinal momentum component $p_{z}$ (relative to its center $p_{0}$ ) will cause the pulse to re-focus in time, which is an equivalent to a transverse static lens (cf. Fig. 3.2). Additional applications include electron guns with high radio-frequency (RF) acceleration fields [114-118], or optimized phase space distributions for time-resolved electron spectroscopy $[119,120]$.

\section{Ultrashort electron pulses from continuous electron beams}

A continuous electron beam can be structured in time, either by cutting the longitudinal phase space distribution, or by bunching in oscillatory fields.

In the first approach, the electron beam is deflected by a transverse RF cavity $[121,122]$, in a plate capacitor like geometry [123, 124] or by fast optical switching [125] of an electric field. Streaking the beam across a subsequent fixed aperture cuts it into short pulses, which can have transverse beam properties comparable to the originating continuous electron beam $[122,126]$. While conserving the peak beam brightness, the charge of a single pulse is intrinsically limited, e.g. an initial probe current of $1 \mathrm{nA}$ and a chopping to $100 \mathrm{fs}$ results in 0.00625 electrons per pulse. Scaling up currents and pulse charge of such a 


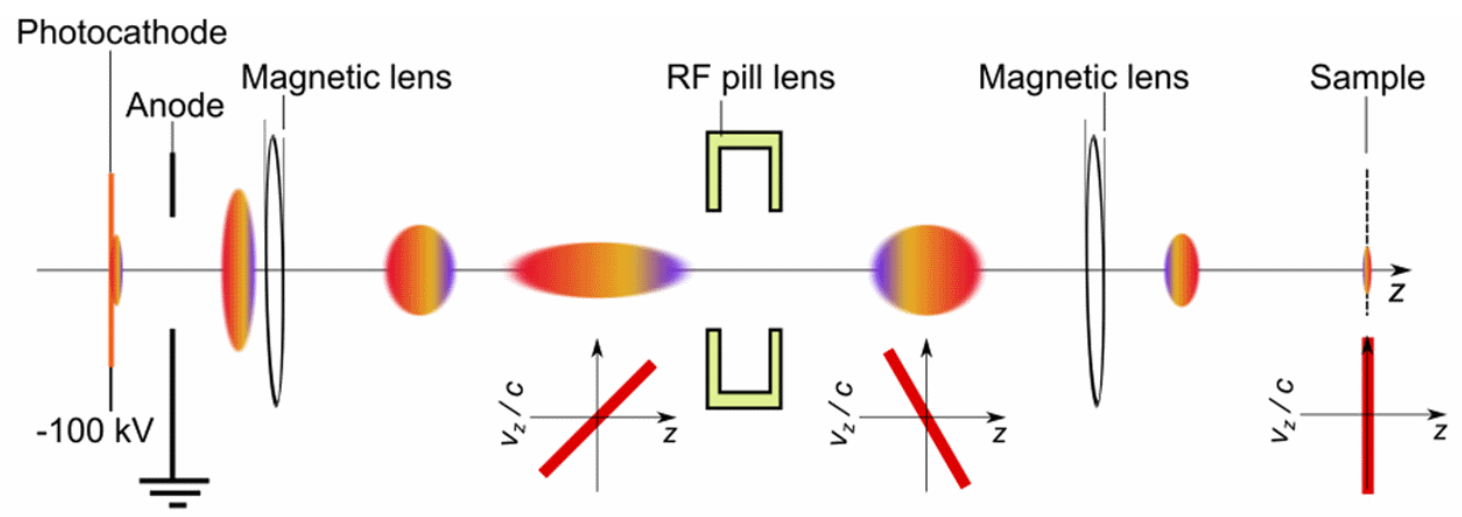

Figure 3.3: Concept of longitudinal RF-compression. Photo-emitted electron bunches are accelerated and guided to an RF compression cavity. The color gradient indicates the electron velocity distribution (red: slow, violet: fast), which is spreading initially due to dispersive propagation. Passing the RF pill lens, the relative electron velocity is inverted along the axis of propagation, creating a phase space distribution that self-compresses at a temporal focal point further down the beamline. Reproduced from [114]. DOI: 10.1088/0034-4885/74/9/096101. ( ) IOP Publishing. Reproduced with permission. All rights reserved.

device is challenging, since an initially low emittance is required to prevent beam quality degradation [126].

Secondly, electrons of a continuous electron beam can be bunched ballistically by an oscillatory longitudinal field, e.g. in a longitudinal RF cavity (cf. Fig. 3.3) which is routinely used in particle accelerator science [127]. At a certain propagation distance, the continous electron beam will evolve into a train of short bunches, with duration given by the amplitude and frequency of the RF-field.

The extension of this concept to the bunching of electron density in single particle wavefunctions in the optical regime and its most crucial experimental prerequisite - the quantum coherent phase modulation of free electrons-is introduced in Ch. 5.

\subsection{Generation of free-electron beams}

The following section covers the generation of continuous and pulsed free-electron beams and briefly introduces the relevant emission mechanisms and electron gun geometries.

Free-electron beams were first observed as "cathode rays" generated from a highly negatively biased electrode in a vacuum tube, as later uniquely identified by Thomson in 1897 [128]. For emitting an electron from a solid, it has to overcome the potential barrier arising from its interaction with the positively charged atomic nuclei. The work function 

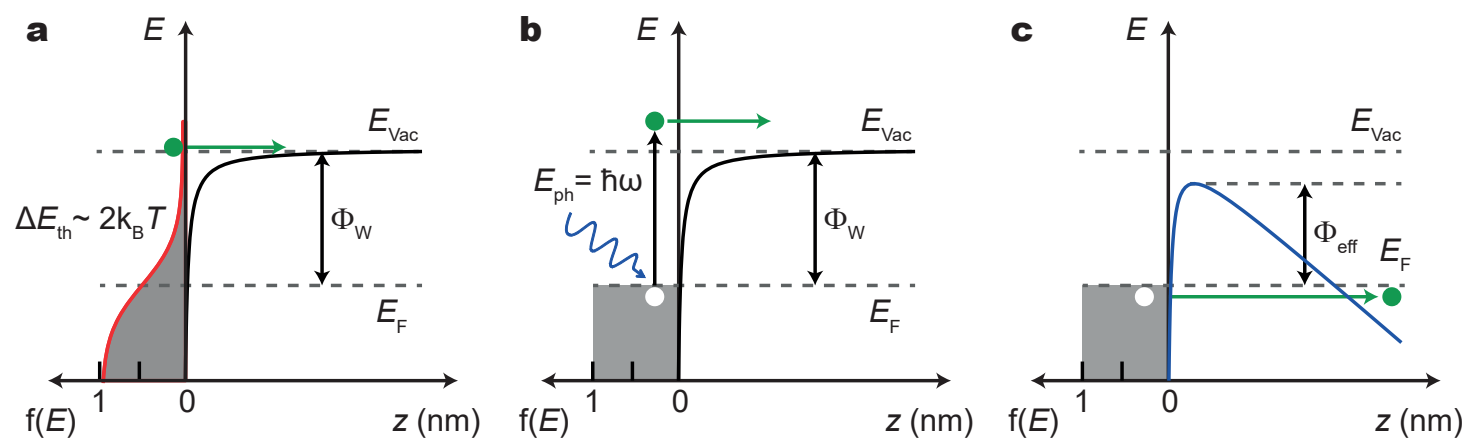

Figure 3.4: Processes of electron emission from solids. The potential barrier involved in an electron being emitted into vacuum can be overcome by (a) thermal excitation, (b) photon absorption or (c) electron tunneling.

$\Phi_{\mathrm{W}}$ gives the minimum work required (at $T=0 \mathrm{~K}$ ) for removing an electron from the solid to an infinite distance in (electric field-free) vacuum. It strongly depends on material, crystal structure and face, as well as the surface configuration of adsorbates. Different types of electron emitters are distinguished by their means of overcoming the potential barrier (thermal excitation, tunneling or photon absorption, cf. Fig. 3.4) and additional effects decreasing the work function (surface configuration, applied electric fields). To compensate for the loss of electrons and prevent charging, conductive materials like metals are most commonly used as electron emitters. For specialized purposes semiconductor emitters are applied, e.g. strained GaAs for generating spin-polarized electron beams [129].

\section{Thermionic electron sources}

Thermal excitation of a solid enables highly energetic electrons to overcome the materials work function $\Phi_{\mathrm{W}}$. The occupation $f(E)$ of energy states $E$ in thermal equilibrium is given by the Fermi-Dirac distribution [130]

$$
f(E)=\frac{1}{\exp \left(\frac{E-\mu}{k_{\mathrm{B}} T}\right)+1},
$$

relative to the chemical potential $\mu$ of a material with Boltzmann's constant $k_{\mathrm{B}}$ and temperature $T$. At high temperatures, and for approximating the emitting area as a uniform metal surface, the Richardson-Dushman equation gives the current density of electrons at 

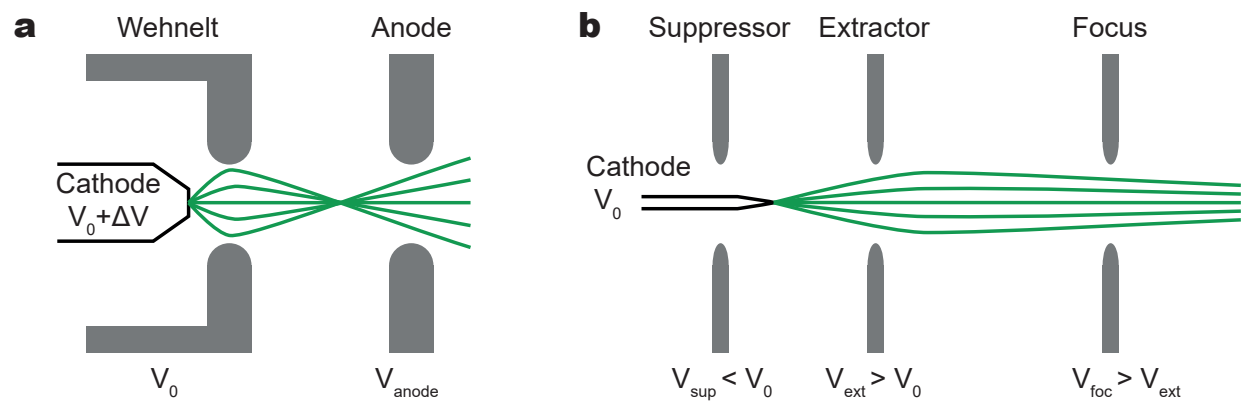

Figure 3.5: Emitter geometries of continuous electron sources. (a) Thermionic emitters employ large emission areas, low acceleration voltages and form a real beam cross-over close to the emitter. (b) Schottky-type emission geometries feature nanoscale electron source sizes, high acceleration fields and minimize space charge impact by forming a virtual beam cross-over (at the emitter). See Ref. [43] for more details.

the high energy tail being emitted into vacuum [130]

$$
j_{\mathrm{T}}=A_{\mathrm{G}} T^{2} \exp \left(-\frac{\Phi_{\mathrm{W}}}{k_{\mathrm{B}} T}\right),
$$

with $A_{\mathrm{G}}=\lambda_{\mathrm{R}} A_{0}$, Richardson constant $A_{0}=4 \pi m k_{\mathrm{B}}^{2} e / h^{3} \approx 120 \mathrm{~A} /\left(\mathrm{K}^{2} \mathrm{~cm}^{2}\right)$ and materialdependent prefactor $\lambda_{\mathrm{R}}<1$. Traditionally, tungsten cathodes $\left(\Phi_{\mathrm{W}}^{\mathrm{W}} \approx 4.5 \mathrm{eV}\right.$ [131]) are employed for thermionic emission, featuring a high thermal stability and a melting temperature of $3650 \mathrm{~K}$. The Arrhenius-like characteristics of Eq. 3.21 cause an exponential rise of current density for a linear increase in temperature, while also strongly broadening the electron energy distribution and initial transverse momentum spread (cf. Eq. 3.4). Mitigating these issues, state-of-the-art thermionic emitter TEMs employ large-area single crystalline $\mathrm{LaB}_{6}$ tips which allow for higher brightness electron beams due to their lower work function $\left(\Phi_{\mathrm{W}}^{\mathrm{LaB}_{6}} \approx 2.7 \mathrm{eV}[132]\right)$.

Thermionic emitters are typically implemented in a triode gun geometry, with the filament, a Wehnelt cap and an anode as electrodes (cf. Fig. 3.5) [43]. Applying a negative bias to the Wehnelt cap reduces the emission area, the beam current and electron source size. The electrode assembly constitutes an electrostatic lens, forming a real beam crossover after the Wehnelt cap. While thermionic electron sources typically yield high current beams, they also possess an undesirable large effective source size and a high transverse momentum spread. Furthermore, electron-electron repulsion at the first beam crossover results in beam degradation (Boersch effect), limiting the overall beam brightness [43]. 


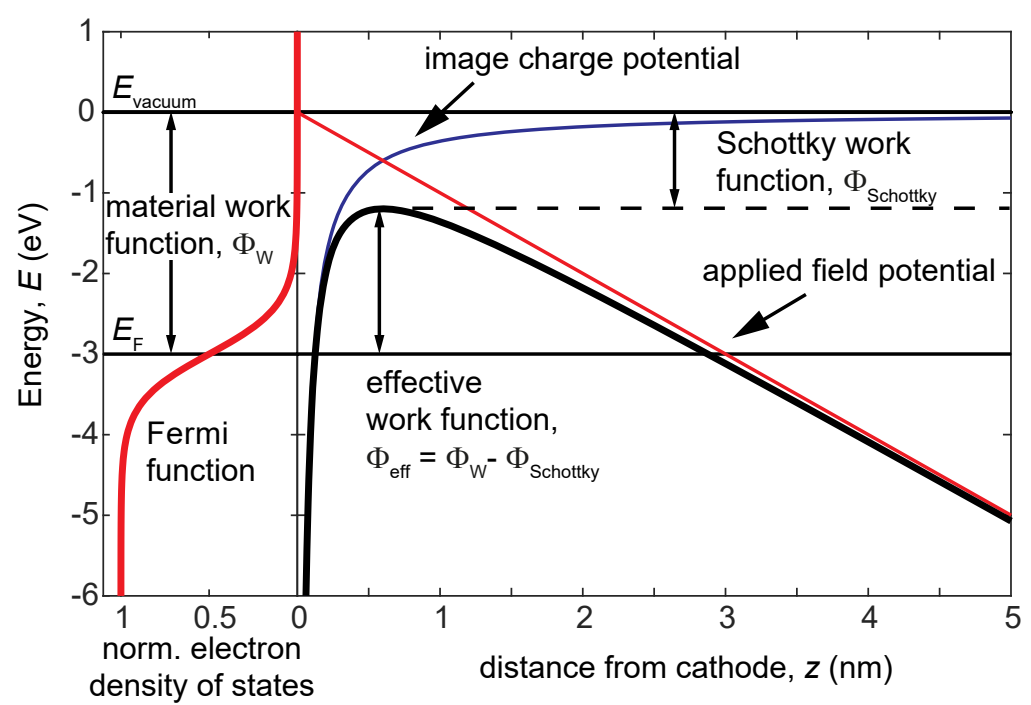

Figure 3.6: Potential landscape of an electron emitter. Total potential (black) includes contributions from the high electric field (red) and the image charge potential (blue) (cf. Ref. [133]).

\section{Field emission sources}

In an alternative approach, field emission electron sources make use of a high static electric field $F$ to modify the potential of an electron leaving the solid (cf. Fig. 3.6) [133]

$$
\Phi(z) \approx \Phi_{\mathrm{W}}-\frac{e^{2}}{16 \pi \varepsilon_{0} z}-e F z
$$

The lowered potential barrier results in the Schottky effect [134], a field-dependent reduction of the effective work function $\Phi_{\mathrm{W}}^{\mathrm{eff}}=\Phi_{\mathrm{W}}-\Delta W$, with $\Delta W=\sqrt{e^{3} F / 4 \pi \varepsilon_{0}}$ [43]. A substantial barrier reduction requires high electric fields in the range of $\sim 1 \mathrm{~V} / \mathrm{nm}$. At the apex of sharp tip emitters, the electric field $F=U_{\text {ext-tip }} /\left(k_{\mathrm{f}} \cdot r_{\text {tip }}\right)$ is geometrically enhanced, with relative potential $U_{\text {ext-tip }}$ of an extraction electrode (at infinite distance), apex radius $r_{\text {tip }}$ and geometry depended correction factor $k_{\mathrm{f}}\left(k_{\mathrm{f}} \approx 5\right.$ for typical opening angles in sphere-on-cone geometry) [135]. 
Cold field emission gun The tunneling current density through a triangular potential barrier can be approximated by the Fowler-Nordheim equation [136, 137]

$$
j_{\mathrm{F}}=\frac{e^{3} F^{2}}{8 \pi h \Phi_{\mathrm{W}}} \exp \left(-\frac{4 \sqrt{2 m_{e} \Phi_{\mathrm{W}}^{3}}}{3 e h F}\right),
$$

assuming zero temperature $T=0 \mathrm{~K}$, a uniform surface and classical electron-electron interaction via an image potential (corrections apply for more realistic potential barriers [137]). This process is referred to as cold field emission since no additional thermal excitation of the electrons is required. Typically, very sharp $(r=10-100 \mathrm{~nm})$ single crystalline tungsten needles $\left((310)\right.$-crystal facet, $\left.\Phi_{\mathrm{W}}^{\mathrm{W}(310)}=4.35 \mathrm{eV}[43]\right)$ are employed at extraction fields of about $F=1-10 \mathrm{~V} / \mathrm{nm}$. The tunneling current is highly sensitive to gas adsorbates and damage caused by sputtering with positively charged, back-accelerated ions. Therefore, despite operation at very low pressures $\left(p=10^{-9}-10^{-10}\right.$ mbar), cold field emitters require regular cleaning by "flashing" the tip at high temperatures [43].

Schottky field emission gun In contrast, the so-called Schottky field emitter makes use of high electric fields and elevated emitter temperatures, combining tunneling and over-the-barrier emission of thermally excited electrons. The field-enhanced thermionic emission, obtained by inserting $\Phi_{\mathrm{W}}^{\text {eff }}$ in Eq. 3.21, is called Schottky emission [43].

For an increasing static electric field, once tunneling contributions become significant, thermally assisted field emission sets in (also called the extended Schottky regime), further modifying Eq. 3.21 to [43]

$$
j_{\mathrm{ES}}=A_{\mathrm{G}} T^{2} \exp \left(-\frac{\Phi_{\mathrm{W}}-\Delta W}{k_{\mathrm{B}} T}\right) \cdot \frac{\pi q}{\sin (\pi q)}
$$

with $q$ being the fraction of the tunneling to the total current [138].

The most commonly employed Schottky-type emitters feature a zirconium oxide ( $\mathrm{ZrO})$ coated (100)-oriented single crystalline tungsten tip with apex radius between $0.1-1 \mu \mathrm{m}$ [138]. A continuous supply of the zirconium oxide is provided by a macroscopic $\mathrm{ZrO}_{2}$ droplet attached to the tip shank and thermally activated diffusion at a temperature of $1700-1800 \mathrm{~K}$. At the (100) front-facet, the high polarizability of the $\mathrm{ZrO}$ overlayer causes a reduction of the work function from $4.55 \mathrm{eV}$ down to about $2.9 \mathrm{eV}$ [139], which is additionally lowered by the Schottky effect. The high temperatures and the self-regenerating 
surface overlayer allow for a stable long-term operation of the Schottky emitter, without the need for regular emitter flashing [138].

In a Schottky emitter, the field emission tip protrudes from a negatively biased suppressor electrode $\left(U_{\text {sup }}<U_{\text {tip }}\right)$ (cf. Fig. 3.5). In such a geometry, the shape of the electric field configuration is characterized by a dimensionless parameter [140]

$$
\Gamma=\frac{U_{\text {tip }}-U_{\text {sup }}}{U_{\text {ext }}-U_{\text {sup }}} \cdot \frac{d_{\text {ext-sup }}}{d_{\text {tip-sup }}},
$$

with $d_{\text {ext-sup }}$ and $d_{\text {tip-sup }}$ being the extractor-suppressor and tip-suppressor distance, respectively. A value of $\Gamma \rightarrow 0$ approximates a geometry without suppressor electrode, while an increasing $\Gamma$ results in a focusing behavior for the electrode assembly, increasing the overall electron transmission through an aperture in the extraction electrode. Additionally, an increasing $\Gamma$ will suppress electron emission from (100)-side facets of the tip shank up to a cutoff point $(\Gamma \sim 1)$, at which even the emission from the apex is prohibited [140] (cf. Sec. 4.3 for more details).

\section{Photoemission electron sources}

The above discussed conventional electron sources make use of a variable degree of thermal over-the-barrier emission and field-assisted tunneling from the tip of a macroscopic emitter. Fast changes in the local temperature of a back-heated filament are limited to the ms-range and above. Gating the emission with a fast modulation of the electric field can shorten those switching times to the ns-range, which still falls short of the requirements of ultrafast science. Combining photoemission by ultrashort laser pulses with existing gun technology enables the generation of high quality, femtosecond-pulsed electron beams, which will be discussed in the following.

\section{Light-assisted electron emission processes}

The classical photoelectric effect, first observed in an experiment by Hertz in 1887 [141] and theoretically elucidated by Einstein in 1905 [142], describes the light-induced electron emission from metal surfaces at low optical fluency (cf. Fig. 3.4b). An electron is emitted from a solid if absorbing the energy of a single photon $E_{\mathrm{ph}}=\hbar \omega$ is sufficient to overcome the work function $\Phi_{\mathrm{W}}$ of the material. This results in a sharp cutoff of the photon energy $\left(E_{\mathrm{ph}}=\Phi_{\mathrm{W}}\right)$ required for emission, an increase of electron kinetic energy $E$ with $\omega$ and an 
linear dependence of the electron current $J$ on the intensity [142]:

$$
E=\hbar \omega-\Phi_{\mathrm{W}}, \quad J_{1 \mathrm{PPE}} \propto I .
$$

This emission regime is called linear, or single-photon photoemission and directly translates a temporal intensity modulation of the light field to the emitted electron current, allowing for the generation of ultrashort electron pulses. At high optical fields, an electron can absorb multiple photons simultaneously, opening up new emission channels for $E_{\mathrm{ph}}<$ $\Phi_{\mathrm{W}}$ (cf. Fig. 3.7a). The final kinetic energy of the electrons depends on $N$ and $E_{\mathrm{ph}}$, while the electron yield scales with the $N$-th power of the intensity [143]:

$$
E=N \hbar \omega-\Phi_{\mathrm{W}}, \quad J_{N} \propto a_{N} I^{N},
$$

with prefactor $a_{N}$ scaling the probability of $N$-photon absorption. The total energy of an electron after emission is $E+N \hbar \omega-E_{\mathrm{F}}-\Phi_{\mathrm{W}}^{\mathrm{eff}}$, which is the initial electron energy after absorption $E+N \hbar \omega$, reduced by the energy of the vacuum state $E_{\mathrm{F}}+\Phi_{\mathrm{W}}^{\text {eff }}\left(E_{\mathrm{F}}\right.$ : Fermi energy) [133]. Therefore, the width of the final electron kinetic energy distribution $\Delta E$ is given by the maximum excess energy $N \hbar \omega-\Phi_{W}$ (electrons emitted from the Fermi energy $E_{\mathrm{F}}$ ) and electrons barely passing over the barrier [144]. While the momentum component $p_{\|}$parallel to the surface is conserved during the emission process, the normal component $p_{\perp}$ is reduced, reflecting the kinetic energy reduction of passing the potential barrier into vacuum. As a consequence, the momentum after emission is not isotropically distributed, but will according to Dowell et al. [133] display a transverse spread of

$$
\sigma_{p_{x, y}}=\sqrt{\frac{N \hbar \omega-\Phi_{\mathrm{W}}^{\mathrm{eff}}}{3 m_{e} c^{2}}} .
$$

In real materials, the total energy spread $\Delta E_{\text {kin }}$ and the transverse momentum distribution $\sigma_{p_{x, y}}$ are further broadened (cf. Fig. 3.7b) by the temperature dependent population of the Fermi-Dirac distribution (e.g., $\Delta E_{\text {thermal }}=2 k_{\mathrm{B}} T \approx 50 \mathrm{meV}$ at $T=300 \mathrm{~K}$ ) [144]. Using ultrashort laser pulses, a further broadening of $\Delta E_{\mathrm{kin}}$ results from the initial non-thermal (and later thermalized) hot electron distribution [145] and the spectral width of the laser [146] (e.g. $\Delta E_{\text {photon }}=60 \mathrm{meV}$ for 30-fs ultrashort laser pulses (FWHM) at $\lambda=800 \mathrm{~nm}$, $\left.E_{\text {photon }}=\hbar \omega=1.55 \mathrm{eV}\right)$. 

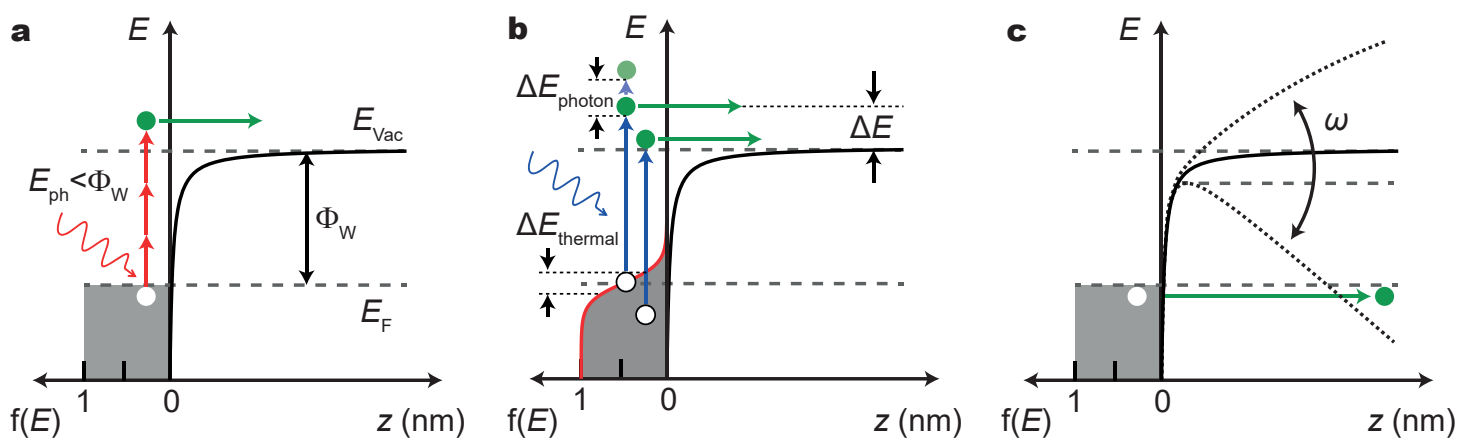

Figure 3.7: Photoemission processes from solids. (a) Multi-photon photoemission at high optical fluences. (b) Contributions to the electron kinetic energy distribution for single photon photoemission, including the excess energy after emission $\Delta E$, the temperature dependent broadening of the Fermi-Dirac distribution $\Delta E_{\text {thermal }}$ and the spectral width of the laser $\Delta E_{\text {photon. }}$ (c) Strong-field photoemission for small Keldysh parameters $\gamma \ll 1$.

At higher fields or longer wavelength, the multiphoton emission process is replaced by strong-field emission, essentially described by the sub-optical cycle tunneling emission through a periodically modified potential barrier and consecutive quivering motion in the alternating field (cf. Fig. 3.7c) [147, 148]. The strong-field regime is assumed for small Keldysh parameter $\gamma=\omega \sqrt{2 \Phi_{\mathrm{W}}} / e F_{\text {peak }} \ll 1$, with peak optical electric field $F_{\text {peak }}$. In the Göttingen UTEM (detailed description in Ch. 4), the electron emitter is illuminated with a peak intensity of $25 \mathrm{GW} \mathrm{cm}^{-2}\left(0.43 \mathrm{~V} \mathrm{~nm}^{-1}\right.$, not regarding optical field enhancement $)$ at $400 \mathrm{~nm}$ wavelength, resulting in $\gamma \gg 1$, therefore excluding strong-field contributions in the emission process [148].

\section{Emitter geometries for ultrafast electron guns}

An electron gun comprises the electron emitter, accelerating elements, beam limiting apertures and possibly additional lenses, forming the final electron beam. The main design considerations for continuous electron guns are [43]:

1. source brightness

2. effective source size (as reduced by apertures)

3. source energy spread and

4. emission stability. 
For sources of ultrashort electron pulses, additional gun properties are of major concern:

5. the electron pulse duration

6. number of electrons per pulse

7. the repetition rate and

8. impact of Coulomb repulsion for high density electron pulses.

Over the last decades, field-emission electron sources proved to deliver the most advantageous trade-off for continuous electron emitters (regarding properties 1.-4.). Nevertheless, pushing for high pulse charge and short pulse durations, the development of ultrafast electron guns mainly focused on the longitudinal phase space (properties 5.-8.), resulting in many optimized variants of planar photocathode gun designs. Here, electrons are photoemitted by ultrashort laser pulses from a macroscopic flat photocathode, accelerated towards an opposing anode at a high positive potential and spatially filtered by subsequent apertures (cf. 4.2). Combining Eq. 3.4 and 3.28 gives a lower bound of the intrinsic transverse source emittance of such a flat photocathode [133]

$$
\varepsilon_{\mathrm{n}, \mathrm{rms}, x}=\sigma_{x} \sqrt{\frac{\hbar \omega-\Phi_{\mathrm{W}}^{\mathrm{eff}}}{3 m_{e} c^{2}}},
$$

with standard deviation $\sigma_{x}$ of the lateral laser focal spot size. For example, typical parameters of $\sigma_{x}=10 \mu \mathrm{m}$ and electron excess energy $\Delta E=\hbar \omega-\Phi_{\mathrm{W}}^{\mathrm{eff}}=500 \mathrm{meV}$, result in an intrinsic emittance of $\varepsilon_{\mathrm{n}, \mathrm{rms}, x}=5 \cdot 10^{-9} \mathrm{~m} \cdot \mathrm{rad}$. In multi-electron pulses, the achievable peak beam brightness is limited by Coulomb repulsion [149]. Strategies for reducing the transverse beam emittance of flat photocathodes are briefly discussed in section 4.3.1.

In conventional electron microscopy, the evolution of high-brightness electron sources resulted in emitter geometries of decreasing electron source size, localizing the electron emission to the apex of nanoscopic tip emitters. The same was recently realized for localized ultrafast photoemission from sharp metal tips [150-152] (cf. Fig. 4.2, 4.3). At these tips (typical apex radius $10-100 \mathrm{~nm}$ ), optical fields are enhanced by a factor $\beta$, resulting in a locally amplified intensity $I_{\text {enhanced }}=\beta^{2} I$. The value of $\beta$ largely depends on the material and tip geometry, with contributions from local plasmonic surface resonances $[153,154]$ and the optical equivalent of the (geometrical) lightning-rod effect (for tungsten: $\beta=3-6$ [155]). Localization of electron emission is achieved by an increased local current density, governed by the nonlinearity of $N$-photon emission (cf. Eq. 3.27). 
The figure-of-merit for localization is the current ratio of electrons being emitted from apex and shaft region [156]

$$
\frac{J_{\text {apex }}}{J_{\text {shaft }}} \propto \frac{2 \pi r_{\text {apex }}^{2}}{\pi r_{\text {laser }}^{2} \cdot \frac{\alpha}{2 \pi}} \beta^{2 N},
$$

with tip opening angles $\alpha$, tip diameter $r_{\text {apex }}$ and laser focal spot size $r_{\text {laser. For the }}$ geometrical conditions used in this work (see Ch. 4) of $r_{\text {apex }}=130 \mathrm{~nm}, r_{\text {laser }}=25 \mu \mathrm{m}$, $\alpha \sim 10^{\circ}$ and assuming a field enhancement of $\beta \sim 3$ (cf. Ref. [155]), results in a degree of localization $J_{\text {apex }} / J_{\text {shaft }}$ of $0.0175(N=1), 0.16(N=2), 1.42(N=3)$ and $12.8(N=4)$, increasing with nonlinearity $n$. Therefore, either very sharp metal tips or a high nonlinearity (e.g. $N=4$ ) are necessary to achieve high-contrast localization, with the downsides of reduced emitter stability [157] and broader kinetic photoelectron energy distribution [147].

In an alternative approach, localized single-photon photoemission from nanoscopic Schottky field emitter tips was recently demonstrated [158, 159]. Here, as described above, the work function of the W(100) crystal-face is reduced, only allowing for linear photoemission from the front facet of the tip. The localization of electron emission $N_{\text {apex }} / N_{\text {shaft }}$ scales with the ratio of single to two-photon emission efficiency.

In the following, Ch. 4 presents an implementation of a UTEM gun, based on a laserdriven Schottky field emitter, that allows for the generation of electron pulses with unique beam properties, combining a low-emittance of down to $\varepsilon_{\mathrm{n}, \mathrm{rms}, x} \sim 2 \cdot 10^{-12} \mathrm{~m} \cdot \mathrm{rad}$ with pulse duration of $200 \mathrm{fs}$ and a narrow spectral bandwidth of $0.6 \mathrm{eV}$. 


\section{Chapter 4}

\section{Ultrafast transmission electron microscopy using a laser-driven field emitter: Femtosecond resolution with a high coherence electron beam}

A. Feist, N. Bach, N. Rubiano da Silva, T. Danz, M. Möller, K. E. Priebe, T. Domröse,

J. G. Gatzmann, S. Rost, J. Schauss, S. Strauch, R. Bormann, M. Sivis, S. Schäfer and

C. Ropers

Ultramicroscopy 176, pp. 63-73, December 2016

DOI: $10.1016 /$ j.ultramic.2016.12.005

We present the development of the first ultrafast transmission electron microscope (UTEM) driven by localized photoemission from a field emitter cathode. We describe the implementation of the instrument, the photoemitter concept and the quantitative electron beam parameters achieved. Establishing a new source for ultrafast TEM, the Göttingen UTEM employs nano-localized linear photoemission from a Schottky emitter, which enables operation with freely tunable temporal structure, from continuous wave to femtosecond pulsed mode. Using this emission mechanism, we achieve record pulse properties in ultrafast electron microscopy of $9 \AA$ focused beam diameter, $200 \mathrm{fs}$ pulse duration and $0.6 \mathrm{eV}$ energy width. We illustrate the possibility to conduct ultrafast imaging, diffraction, holography and spectroscopy with this instrument and also discuss opportunities to harness quantum coherent interactions between intense laser fields and free-electron beams. 


\subsection{Introduction}

The continuing advancement of electron microscopy within physics and chemistry, materials science, and structural biology $[66,160,161]$ provides us with ever-increasing precision in viewing structure and composition on the nanoscale. A detailed microscopic understanding of the structural, electronic and magnetic properties of natural and synthetic materials demands - besides atomic-scale spatial characterization - the investigation of the response of these systems to external perturbation. The growing importance of in-situ approaches in transmission electron microscopy [162], scanning electron microscopy [163], X-ray diffraction [164], scanning tunneling and atomic force microscopy [165], and other areas testify to this development.

Time-resolved experiments, following the dynamical response of a system to a pulsed excitation, represent an especially powerful form of in-situ probing, which yields direct time-domain access to the character and strengths of the couplings between structural, electronic and spin degrees of freedom. Ultrafast electron [25, 115, 116, 166-168] and Xray [26, 169-171] diffraction are well-established techniques to track structural relaxation with femtosecond temporal resolution, widely applied to homogeneous and thin film systems. The observation of spatiotemporal relaxation processes in heterogeneous systems [172-177], however, such as excitation and energy transfer across functional interfaces, is particularly challenging, requiring simultaneous nanoscale spatial and ultrafast temporal resolutions. To this end, various experimental approaches are pursued very actively at present, including time-resolved variants of scanning tunneling microscopy (STM) [27, 178, 179] and scanning near-field optical microscopy (SNOM) [28, 180, 181]. Furthermore, imaging techniques using ultrashort electron pulses such as compact point-projection electron imaging [29, 182-184] and ultrafast scanning electron microscopy [124, 159, 185-187] are being developed.

Beyond these approaches, ultrafast transmission electron microscopy (UTEM) promises to become one of the most powerful experimental tools for the investigation of ultrafast dynamics on the nanoscale, joining femtosecond temporal resolution with the vast opportunities in imaging, diffraction and spectroscopy provided by state-of-the-art electron optics. Early pioneering works at the Technical University Berlin [30], Caltech [32] and Lawrence Livermore National Labs [47] demonstrated the feasibility of pump-probe studies in electron microscopy, either in a stroboscopic fashion [82] or using single-shot imaging [31]. Motivated by various notable individual results highlighting its broad po- 
tential, time-resolved electron microscopy is currently explored in a growing number of laboratories worldwide [34, 188-193].

Being considered one of the most exciting frontiers in electron microscopy, the area of ultrafast transmission electron microscopy is presently at a pivotal moment of its development. Facing great challenges in obtaining intense high-quality electron pulses, time-resolved electron microscopy is in particular need of benchmarking the currently achievable spatio-temporal resolution limits and electron beam figures-of-merit. Quantitative characterizations will be required to facilitate systematic progress and to connect this emerging field to the well-established, powerful experimental and theoretical framework of electron microscopy $[39,194]$. In order to harness the full imaging and spectroscopy capabilities of today's electron microscopes, it is highly desirable to integrate higherbrightness pulsed electron guns into the electron optics environment of a transmission electron microscope, in particular based on laser-triggered field emitter concepts. Not unlike the scientific leaps that are associated with technological breakthroughs in bright continuous electron sources in the past $[36,41]$, significant advances in pulsed electron source quality promise a path to uncharted territory in ultrafast nanoscale dynamics.

In this contribution, we describe the first implementation of an ultrafast transmission electron microscope based on laser-triggered electron emission from a nanoscale photocathode (Ch. 4.2). We provide a quantitative characterization of the spatial and temporal electron beam properties for a variety of electron-optic illumination conditions (Ch. 4.3), demonstrating electron pulse durations down to $200 \mathrm{fs}$, energy widths of $0.6 \mathrm{eV}$ and a focusability of the photoelectron beam to sub-nm dimensions. We illustrate a range of possible applications for this instrument, which include bright- and dark-field imaging, convergent beam electron diffraction (CBED) from nanoscale areas, phase-contrast imaging and Lorentz microscopy, holography and spatially-resolved electron spectroscopy (Ch. 4.4). Beyond adding femtosecond temporal resolution to this set of conventional electron microscopy techniques, the advanced electron beam properties of the field-emitter UTEM render it ideally suited to be applied in contrast mechanisms and phenomena that are exclusive to ultrafast electron microscopy, such as photon-induced near-field electron microscopy (PINEM) or the quantum coherent manipulation of free-electron beams (Ch. 4.5). 


\subsection{Instrumentation}

Ultrafast transmission electron microscopy is a stroboscopic imaging technique, in which dynamics in an investigated sample are triggered by short (typically optical) excitation pulses. At well-defined delay times after excitation, the evolving state of the sample is probed by an ultrashort electron pulse (Fig. 4.1a). Accumulating, for a given delay time, the signal derived from many electron pulses yields a stroboscopic snapshot of the transient state of the system $[47,166]$. Importantly, the temporal resolution of such a pump-probe approach is given by the electron pulse duration and is not limited by the speed of the electron detector. In the past, employing photoemission driven by ultrashort laser pulses has enabled the generation of electron bunches with femtosecond duration, which are now finding increasing use in time-resolved electron imaging, diffraction and spectroscopy techniques [32, 34, 116, 166-168, 174, 195-197].

The Göttingen UTEM instrument is based on a JEOL JEM-2100F transmission electron microscope, which was modified to allow for both optical sample excitation and pulsed electron emission. In contrast to previous implementations of time-resolved TEM, we employ a laser-driven Schottky emitter, which confines the photoemission to the nanoscale front facet of a $\mathrm{ZrO} / \mathrm{W}$ emitter tip $[158,159]$. The emitter is side-illuminated with $400 \mathrm{~nm}$ laser radiation, focused to a spot diameter of about $20 \mu \mathrm{m}$ full-width-at-half-maximum (FWHM). Optical access to the emitter tip is given through a side window on the TEM gun and by a further optical steering assembly inside the ultrahigh vacuum chamber of the electron source. For time-resolved experiments, frequency-doubled femtosecond optical pulses from a regenerative Ti:Sapphire amplifier (Coherent RegA) are used at pulse energies of about $10 \mathrm{~nJ}$ and at a tunable repetition rate of up to $800 \mathrm{kHz}$. For alignment of the photoelectron beam into the TEM electron optics, and for characterization of electron beam properties in the space-charge-free regime, a continuous diode laser is employed at an average optical power of typically $20 \mathrm{~mW}$. Utilizing the usual field geometry of a continuous Schottky source, the laser-triggered emitter is placed into an electrostatic suppressor-extractor electrode assembly, characterized by a dimensionless parameter $\Gamma$ [140], which allows for tuning the extraction field at the emitter apex and the divergence of the photoelectron beam. (For further details on tailoring photoelectron beams in a Schottky emitter assembly, see Ref. [140]). Electrons far from the optical axis are cut by a hard aperture, which is placed in the electrostatic gun lens. By changing the voltages applied to these three electrodes, the electron gun can be operated in different modes, e.g., 

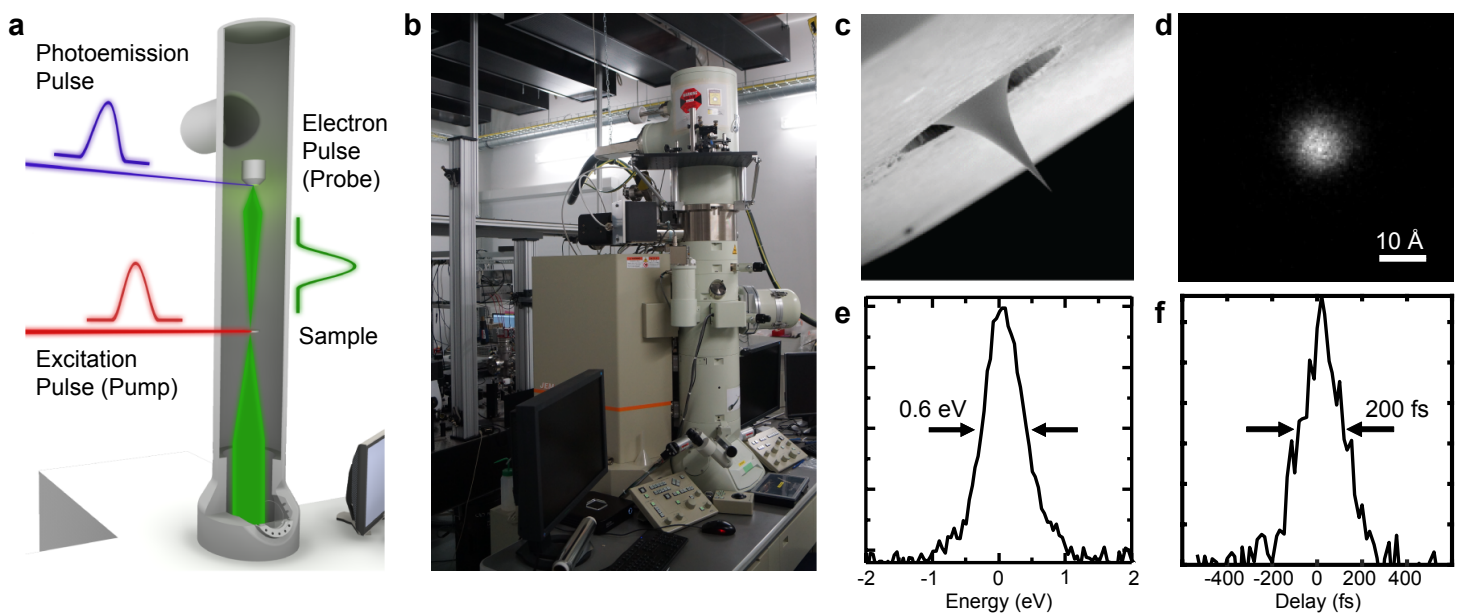

Figure 4.1: Schematic setup and electron pulse properties of the Göttingen UTEM instrument. A laser-driven Schottky field emission electron gun (a) is combined with the column of a JEOL JEM-2100F (b). Side illumination of a nanoscopic ZrO/W(100) tip emitter (c) enables the generation of ultrashort electron bunches, which can be focused down to $0.89 \mathrm{~nm}$ (d), with an energy width of $0.6 \mathrm{eV}$ (e) and a duration of $200 \mathrm{fs}$ (f) (apertured beam, at $200 \mathrm{kV}$ acceleration voltage).

optimized for a high electron yield or a high beam coherence (cf. Ch. 4.3.2). Finally, after acceleration up to $200 \mathrm{keV}$, the probing electron beam is formed by the condenser system of the TEM column.

For optical sample excitation, we devised two optical beam paths by inserting mirror assemblies into the TEM column. First, access through a port conventionally used for adding an energy-dispersive X-ray spectrometer allows for optical excitation at an angle of incidence of $55^{\circ}$ relative to the electron beam. Second, excitation close-to-parallel to the electron beam is provided by an illumination through the objective lens pole piece. For both cases, typical optical focal spot sizes on the sample at a central wavelength of $800 \mathrm{~nm}$ are about $50 \mu \mathrm{m}$ FWHM. The delay time of optical excitation, optical fluence, polarization state and focal spot position can be changed in an automatized fashion, allowing for versatile means to investigate the dependence of sample dynamics on the optical excitation. 


\subsection{Implementation of a laser-triggered field emitter in the UTEM}

\subsubsection{Localized photoemission from needle-shaped photocathodes}

Over the past decade, various approaches have been undertaken to enhance the beam quality of ultrafast electron sources for time-resolved experiments, including a tailoring of the photoemission laser wavelength [198], photocathode work functions and materials [133, 199, 200], as well as photoemission spot sizes [201, 202], and by enhanced extraction fields in radio-frequency cavities [116] and at sharp needle emitters [174]. Also alternative approaches using cold atomic gases are pursued [203-205].

The nanoscale localization of the emission area in needle-shaped laser-driven photocathodes promises particularly coherent electron beams and has thus been intensely studied recently [147, 150-152, 157, 206-213]. Compared to beams derived from state-of-the-art planar photocathodes $[115,133,146,166,198,201,214]$, electron pulses emitted from tip emitters occupy a significantly reduced area of transverse phase space (cf. Fig. 4.2). In particular, providing the same advantages as static field emitters for conventional electron microscopy [39, 41], ultrafast nanoscale electron sources are crucial for time-resolved imaging applications, which require either a sharply focused or a well-collimated, highly coherent electron beam (cf. Fig. 4.6, Chap. 4.4). Consequently, they have enabled ultrafast low-energy electron diffraction [174], ultrafast scanning electron microscopy [159, 187] and fs-point projection imaging [29, 182-184, 215, 216], and, as we demonstrated recently, ultrafast transmission electron microscopy with highly coherent electron beams [34].

In tip-based ultrafast photoemission, localization is typically achieved by employing optical field enhancements at the apex in combination with a high nonlinearity of the photoemission process [147, 148, 150-152, 183, 184, 206]. However, processes such as higher-order multi-photon and strong-field photoemission exhibit significantly broadened photoelectron energy distributions [147, 148], which limits their use for ultrafast electron imaging and spectroscopy applications. In a regime of lower nonlinearity, sufficient beam qualities can be achieved by photoemission partially localized at the emitter apex in combination with a small electron energy bandwidth, as recently demonstrated using two-photon photoemission (cf. Fig. 4.3h) from tungsten needle emitters [34, 140, 174]. In principle, a linear photoemission regime may be considered ideal, due to the low thermal load on the emitter as well as a simple tunability of the electron pulse length via the laser pulse duration, provided nanoscale localization is ensured. 

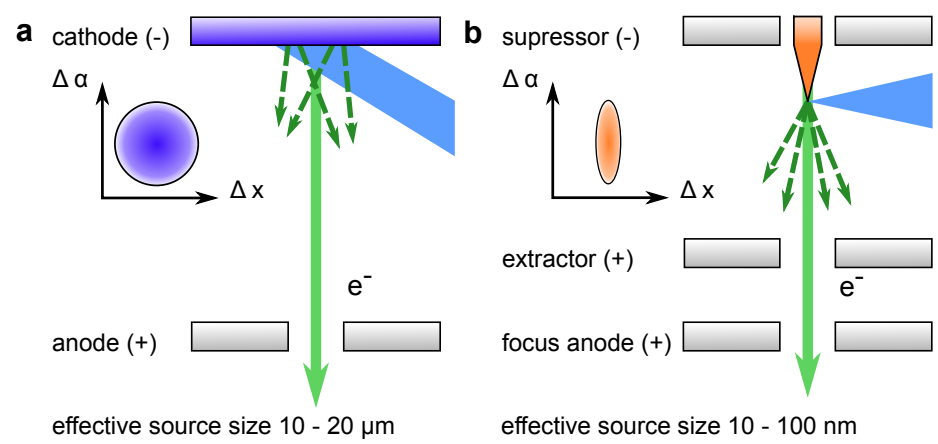

Figure 4.2: Transverse beam properties of photoelectron beams emitted from laser-triggered electron sources. (a) The micrometer-scale emission area (typical diameter of $10-20 \mu \mathrm{m}$, governed by the laser spot diameter) of a flat photocathode results in photoelectron bunches occupying a considerable transverse phase space volume. (b) Localized electron emission from the apex of a nanoscopic tip (typical apex diameter of $10-100 \mathrm{~nm}$ ) allows for a minimization of the phase space volume occupied by the photoelectrons, resulting in significantly enhanced beam coherence. Insets: Sketch of the transverse phase space distributions for the respective source geometries ( $\Delta x$ : spatial coordinate perpendicular to beam propagation axis, $\Delta \alpha$ : angular coordinate relative to the optical axis).

In this work, we introduce a linear photoemission process for UTEM, in which photoemission is localized by chemically tuning the work function of the emitter front facet. Specifically, we make use of single-photon photoemission from zirconium oxide covered (100)-oriented single crystalline tungsten tips ( $\mathrm{ZrO} / \mathrm{W}(100)$, cf. Fig. 4.3a-d) [158, 159] and quantitatively characterize their performance in UTEM.

Schottky field-emitters based on $\mathrm{ZrO} / \mathrm{W}(100)$ without laser excitation are routinely used and well-characterized as continuous electron sources with high brightness [138]. Operated at an elevated temperature of about $1800 \mathrm{~K}$ and with electric extraction fields in the range of $0.5-1 \mathrm{~V} / \mathrm{nm}$, the (100) front facet of the emitter is covered by a $\mathrm{ZrO}$ overlayer, which reduces the work function down to about $2.9 \mathrm{eV}$. A further lowering of the work function is achieved by the applied extraction field (Schottky effect), resulting in intense thermal electron emission from such sources [217, 218] (cf. Fig. 4.3f).

In photoemission mode, we operate the emitter at a reduced temperature (below 1400 K), so that static electron emission is fully suppressed, and photoelectrons are only generated with the laser focus placed at the tip apex. At an illuminating wavelength of $400 \mathrm{~nm}$, the extracted photocurrent scales linearly with the incident laser power (Fig. 4.3i), signifying a single-photon photoemission process (Fig. $4.3 \mathrm{~g}$ ), and the strong localization of photoemission at the (100) front facet is demonstrated by imaging the photoelectron source using the 

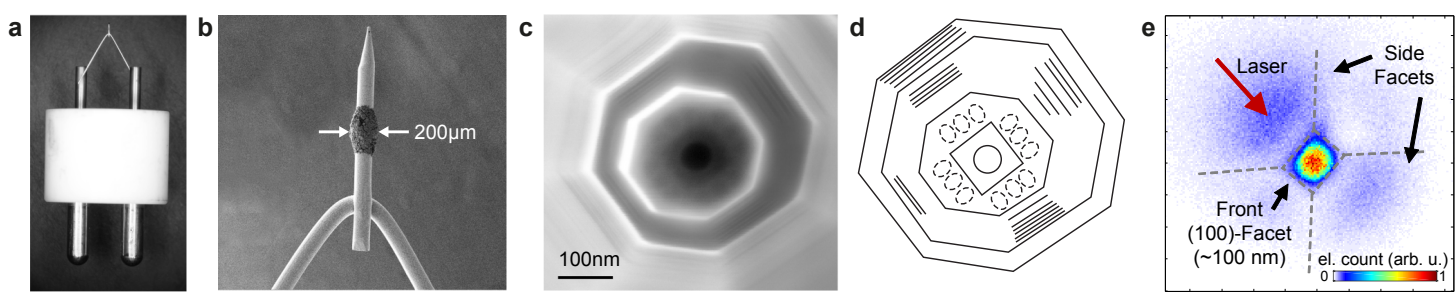

f
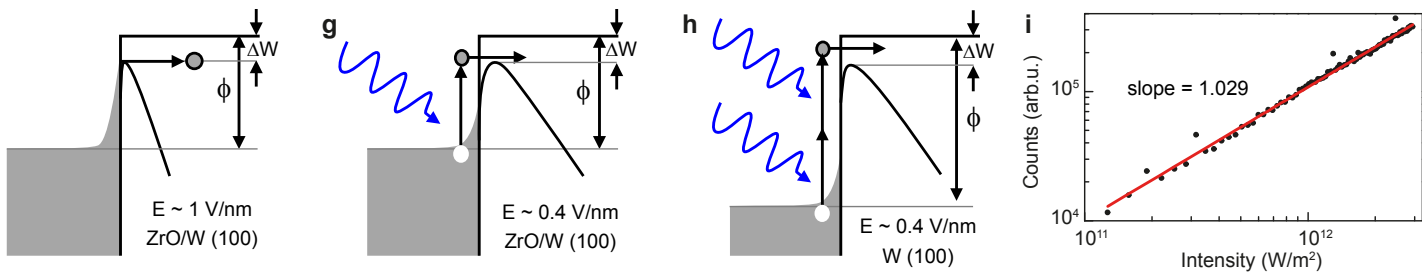

Figure 4.3: Operation principle of a laser driven Schottky field emitter and its geometry. (a and b) Single-crystalline, (100)-oriented tungsten tip emitter attached to a heating filament. The $\mathrm{ZrO}_{2}$ reservoir deposited on the emitter shank is visible in (b). (c and d). Top-view SEM micrograph (c) and corresponding schematic (d) of the faceted apex shape after high-temperature tip conditioning. (e) Photoemission pattern demonstrating the localized photoemission from the (100) front facet (central intense spot) with minor contributions from (100)-equivalent crystal surfaces at the emitter shaft (weak intensity side-lobes). (f-h) Schematic energy diagrams at a Schottky-lowered potential barrier for continuous thermal electron emission (f), and linear (g) and two-photon (h) photoemission. (i) The photoemission current scales linearly with incident laser power density, as expected for a single-photon photoemission process.

TEM optics (Fig. 4.3e).

The photoemitter can be operated continuously and stable for an extended period of time $(>48 \mathrm{~h})$ at high photoelectron currents. Over time, a slow decrease in photoemission efficiency is observed; however, the primary emission current can be fully recovered by flashing the tip to temperatures above $1700 \mathrm{~K}$. Further utilizing these emitters in electron microscopy requires a detailed analysis of their performance in an electrostatic lens assembly [140, 206] and of the resulting beam properties in the TEM column, which we will address in the following.

\subsubsection{Characterization of spatial beam properties using photoelectrons}

The quality of an electron beam, or radiation source in general, for imaging, local probing or diffraction is commonly assessed by its normalized, time-averaged brightness $B_{n}$, which, 
at the center of a Gaussian-shaped beam, is given by [103]:

$$
B_{n}=\frac{I}{4 \pi^{2} \varepsilon_{n, r m s}^{2}},
$$

where $I$ denotes the total beam current. The quantity $\varepsilon_{n, r m s}$ is the so-called normalized r.m.s. (root-mean-squared) emittance [101], which describes the density-weighted transverse phase space area occupied by the particle beam, and accounts for its transverse coherence properties. The normalized beam brightness is a conserved quantity for aberration-free optics, unaffected by apertures and imaging lenses (for definitions of the emittance purely relying on areas in phase space instead of phase space density distributions, also aberrations preserve the corresponding brightness [105]). Space-charge interactions within the (pulsed) electron beam generally result in a decrease of beam brightness [101, 219]. In order to quantitatively characterize the brightness of the UTEM photoelectron beam for different source and condenser settings, we measure the caustic of the focused beam in the sample plane. The electron beam current is recorded with a calibrated CCD camera, and the emittance is derived from the width of the electron focal spot and the beam convergence angle.

Specifically, for a focused beam, the normalized r.m.s. emittance along one transverse direction $(x)$ is given by [101]:

$$
\varepsilon_{n, r m s, x}=\beta \gamma \cdot \sigma_{x} \sigma_{\alpha x},
$$

with $\beta=v / c$ ( $v$ : electron velocity, $c$ : light velocity) and the Lorentz factor $\gamma$. Here, the width of the focused electron beam along the $x$-axis and its corresponding distribution in propagation angles are characterized by the standard deviations $\sigma_{x}$ and $\sigma_{\alpha x}$, respectively. The obtained focal spots are largely symmetric along the two transverse directions ( $x$ and $y$ ). Small asymmetries are accounted for by defining the overall emittance $\varepsilon_{n, r m s}$ as the geometrical mean value of the emittance along the $x$ - and $y$-direction.

At different condenser settings, the spatial beam size in the sample plane is shown in Fig. 4.4a, varying the excitation strength of the second magnetic lens in the condenser system (CL3, cf. Fig. 4.4b) and thus placing the electron focus at different positions relative to the sample plane [220, 221]. An effective beam caustic, i.e. the beam envelope as a function of the position along the electron optical axis (Fig. 4.4c), is then extracted by taking into account the beam convergence angle measured in diffraction mode. The 
a

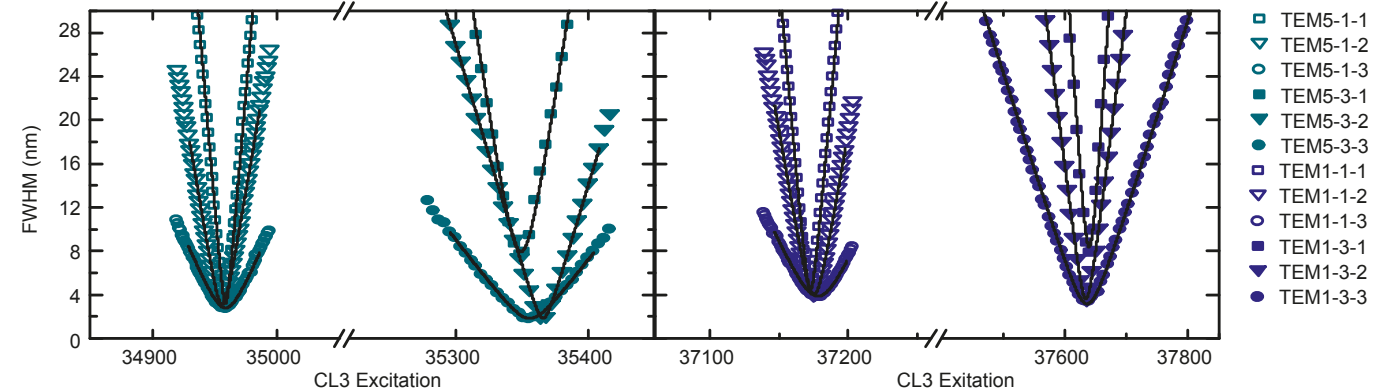

b
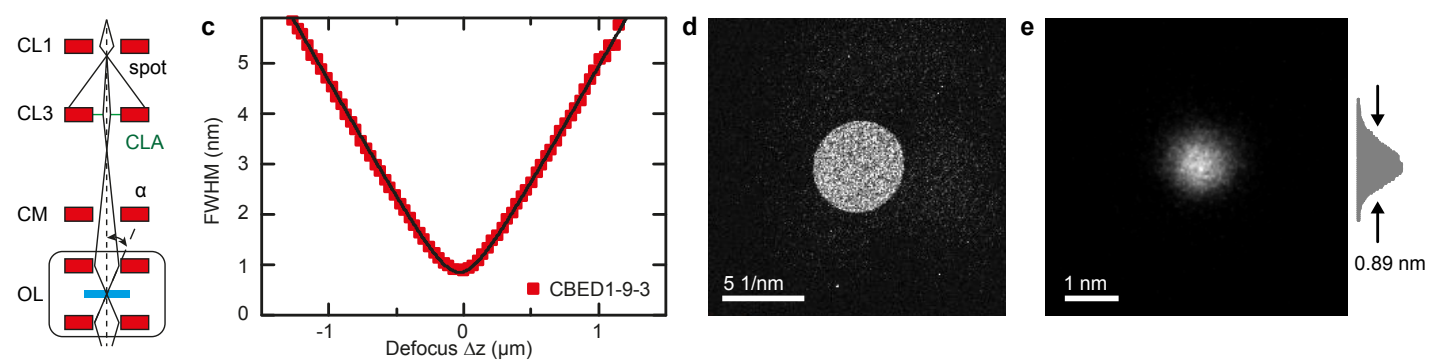

Figure 4.4: Characterization of the transverse electron beam properties. (a) Beam caustics for a range of condenser settings 'TEM a-b-c', with transverse beam coherence 'a' (spot size), convergence angle ' $b$ ' (alpha) and condenser aperture 'c' (CLA) in TEM illumination mode. (b) Illustration of the TEM column illumination system. The beam coherence is adjusted by the first condenser lens (CL1), the convergence angle is set by the condenser mini lens (CM) and the beam is limited by the condenser aperture (CLA). The second condenser lens (CL3) is adapted accordingly to form a focus at the sample plane. (c) Beam diameter in the focal plane of the imaging system (extracted from the standard deviation considering a Gaussian-shaped beam profile) as a function of the second condenser lens excitation CL3. (d and e) Angular distribution and beam profile at the minimum focal spot. ((b-d): convergent beam illumination mode (CBED)).

exemplary caustic shown in Fig. $4.4 \mathrm{c}$ exhibits a minimum focal spot size $d_{\text {min }}$ of only $0.89 \mathrm{~nm}$ at a convergence semi-angle $\alpha$ of $6.7 \mathrm{mrad}$ (cf. Fig. 4.4d,e). For this setting, we obtain an r.m.s. emittance of the photoelectron beam of only $1.71 \mathrm{~nm} \cdot \mathrm{mrad}-\mathrm{a}$ value which is only one order of magnitude larger than the minimum emittance governed by the uncertainty principle, easily derived as $\varepsilon_{n, r m s}=\hbar /\left(2 \cdot c \cdot m_{0}\right)$ - and a temporally averaged, normalized brightness of $2.85 \cdot 10^{7} \mathrm{~A} /\left(\mathrm{m}^{2} \mathrm{sr}\right)$.

Considering the transverse coherence length [111] given by

$$
\xi_{c, x}=\frac{\hbar}{m_{e} c} \cdot \frac{\sigma_{x}}{\varepsilon_{n, r m s, x}}
$$

such a beam readily allows for $1-\mu \mathrm{m}$-scale coherence lengths (cf. Fig. 4.7g) when spread 
to a diameter of about ten micrometers (FWHM). The degree-of-coherence $K=\xi_{c, x} /\left(2 \sigma_{x}\right)$ [109] is $11.2 \%$, which, equivalently, can be described by a beam quality factor of $M^{2}=1 / K=8.9$.

In UTEM, the beam current, emittance, and degree-of-coherence can be precisely tailored, depending on the relative potentials applied to the suppressor and extractor electrode in the electron source, and the settings of the condenser system (selected conditions shown in Table 4.1). In particular, for an extractor-suppressor field geometry ( $\Gamma=1.11$, see Ref. [140]) allowing for a high transmission through the beam-limiting aperture in the source, a beam current of about $460 \mathrm{fA}$ is obtained with a focal spot size of $28 \mathrm{~nm}$, whereas in a high-coherence mode ( $\Gamma=0.55)$, local probing with sub-nm spot sizes is achieved.

To compare our laser-driven emitter with a usual continuous electron source, we derive a normalized peak brightness $B_{n p}$ by scaling the time averaged brightness with an effective duty cycle $D_{\text {eff }}=\sqrt{2} \cdot f \cdot \sigma_{t}$ containing the laser repetition rate $f$ and a typical FWHM electron pulse duration $\tau=\sqrt{8 \ln (2)} \cdot \sigma_{t}$ at the sample, i.e. $B_{n p}=B_{n} / D_{\text {eff. }}$. For operation in regimes not affected by space charge, we arrive at a normalized peak brightness of $1.75 \cdot 10^{13} \mathrm{~A} / \mathrm{m}^{2} \mathrm{sr}$, which is comparable to reported time-averaged values for a conventional Schottky field emitter [217, 218].

For multi-electron pulses, longitudinal and transverse space-charge broadening can be observed, although the effect on the transverse beam properties is rather moderate. In particular, even for electron pulses spectrally broadened to about $5 \mathrm{eV}$, we only observe a slight degradation of the transverse beam properties (cf. Section 4.3.3 and Fig. $4.5 \mathrm{~d}$ and h). Generally, we note that space-charge effects at nanoscopic tip emitters are expected to be reduced compared to flat photocathodes, due to the high intrinsic extraction fields $(\sim 1 \mathrm{~V} / \mathrm{nm})$ and divergent beam trajectories.

\subsubsection{Characterization of temporal electron bunch properties}

Electron pulse durations are experimentally determined by laser-electron cross-correlation [90, 100, 222-227]. In particular, as discussed in more detail in Ch. 4.5, electrons which traverse an intense optical near-field experience inelastic scattering, resulting in photon sidebands in the electron energy spectrum $[34,90]$.

Figures 4.5(a and e) show electron energy spectra as a function of the delay between optical near-field excitation and electron arrival time at the sample, for two experimental conditions differing in the number of electrons per pulse (gun operated in high-coherence 


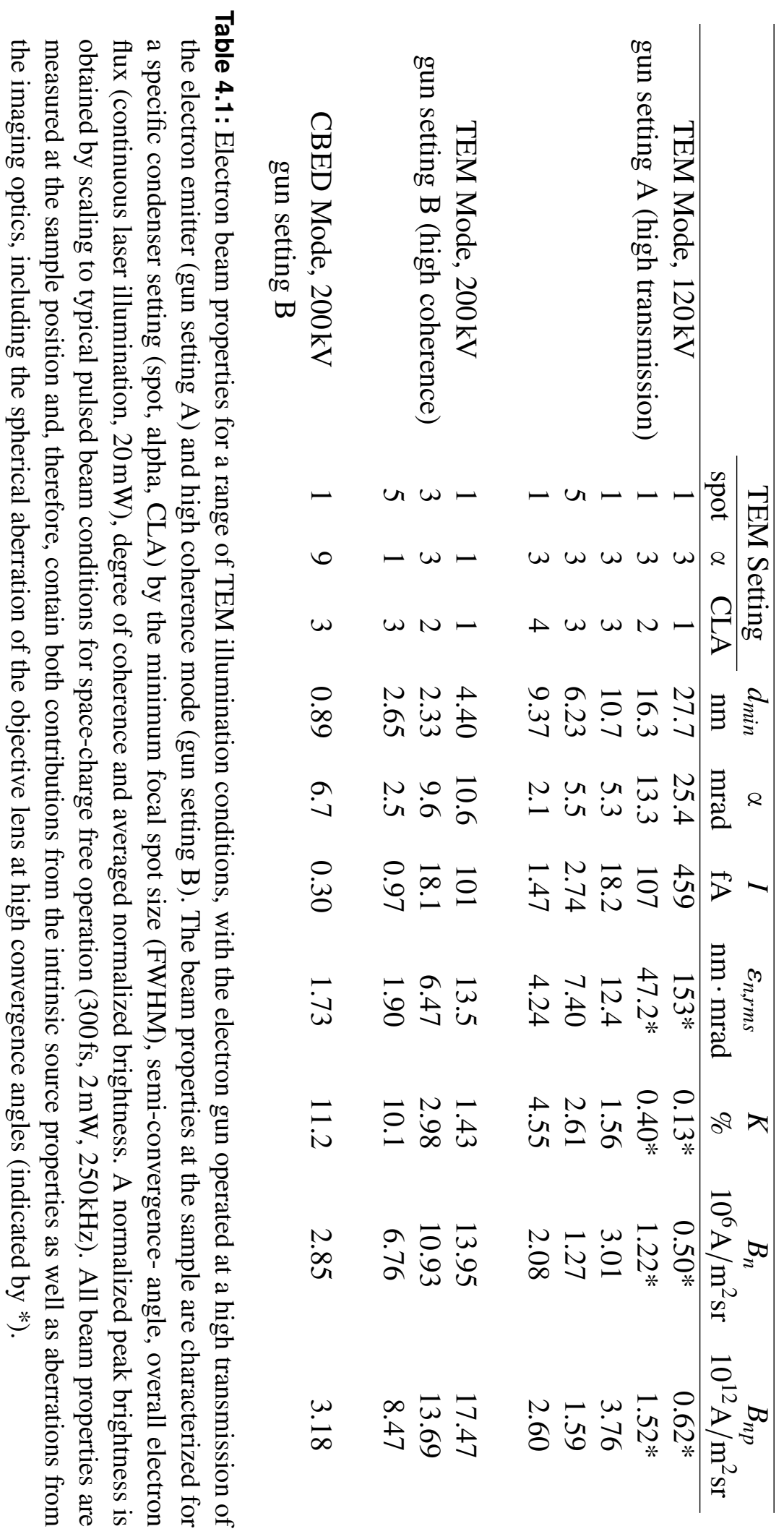


mode). In the first case, with minimized space-charge broadening (Fig. 4.5a), photon sidebands on the energy-gain and -loss side are visible only within a narrow delay window around the temporal overlap between electron and laser pulses. It should be noted that due to the convolution of the electron pulse profile with the nonlinear electron-light interaction across the 50-fs optical pulses, the temporal interval with considerable photon sideband intensity represents a reliable upper bound to the electron pulse duration [34, 100, 225]. Hence, the total number of gain-scattered electrons for different delay times (Fig. 4.5c) yields a measurement of the electron pulse duration of $200 \mathrm{fs}$, the shortest value reported to date in time-resolved transmission electron microscopy.

For electron pulses containing a larger number of electrons at emission, space-charge effects become operative, accelerating electrons at the leading edge of the bunch, and decelerating electrons at the trailing end [228]. As a result, the electron bunch width is temporally broadened, illustrated by the appearance of photon sidebands over a delay interval of about $1 \mathrm{ps}$ (Fig. 4.5e and $\mathrm{g}$ ) and by the increase in the energetic width of the zero-loss peak from $0.85 \mathrm{eV}$ to about $3.5 \mathrm{eV}$. The associated pronounced chirp of the electron pulse is visible as an inclination of the individual sidebands in the electron energy maps (Fig. 4.5e) [100, 229]. Averaging over each sideband in the delay-energy maps gives a direct representation of the longitudinal phase space structure of the electron bunch (Fig. 4.5b and f). Specifically, for the space-charge broadened pulse considered here, we extract a chirp of $275 \mathrm{fs} / \mathrm{eV}$ and a momentary energetic width of $0.65 \mathrm{eV}$ (measured for a single delay value), close to the overall spectral width of the nonbroadened pulse. Such a strong correlation between the longitudinal electron position (i.e. arrival time at the sample position) and electron energy indicates that space-charge forces predominantly lead to shearing in the longitudinal phase space, approximately preserving the bunch's phase space volume. Therefore, in future UTEM implementations, phase-locked radio-frequency [230] or $\mathrm{THz}[116,231]$ fields may be incorporated into the TEM column to temporally and spectrally re-compress multi-electron bunches, as already successfully applied in ultrafast electron diffraction beam lines [116, 214, 229, 232].

While space-charge induced deterioration of transverse beam properties presents a major challenge for the high bunch charges in single-shot imaging [47, 115, 233, 234], space charge only weakly affects the few-electron pulses studied here. Specifically, the minimal focal spot size displays only a minor increase, when comparing thermal and spectrally strongly broadened photoemission, here from $2.2 \mathrm{~nm}$ to $3.5 \mathrm{~nm}$ with an energy width increase from $0.7 \mathrm{eV}$ to $5.3 \mathrm{eV}$ (cf. Fig. $4.5 \mathrm{~d}$ and $\mathrm{h}$ ). We note that the electron bunches 
a

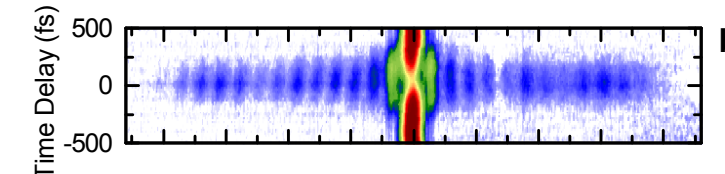

e

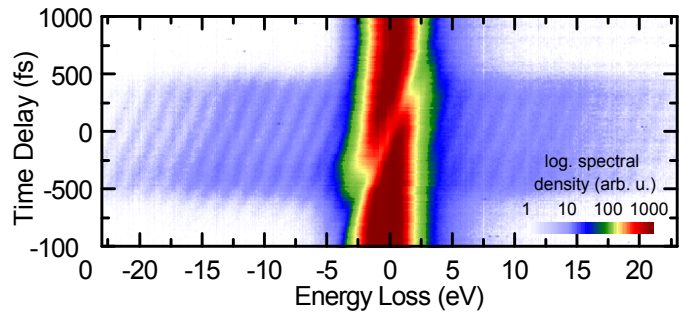

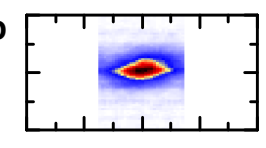

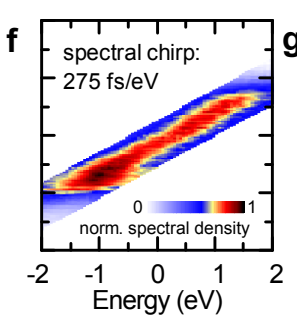

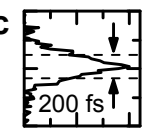

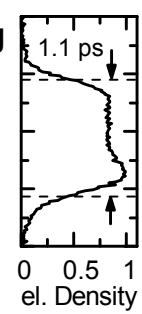

$=$

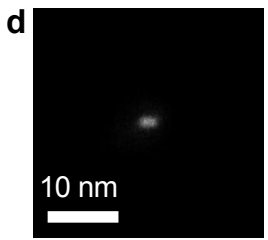

$\mathrm{h}$

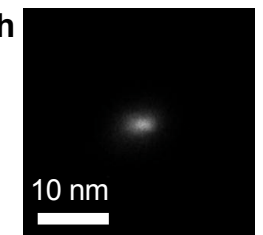

Figure 4.5: Temporal pulse characterization. Electron pulse durations measured by electron-photon cross-correlation in the absence $(\mathrm{a}-\mathrm{c})$ and presence $(\mathrm{e}-\mathrm{g})$ of space-charge broadening (about 100-times higher pulse charge in (e-g)). (a and e) Electron energy-loss spectra as a function of time delay (logarithmic color scale). (b and f) Extracted energy- and time-resolved structure of electron pulses, revealing a linear chirp in the case of space-charge broadened electron bunches and a longer pulse duration (c and g). Comparing the focal spot sizes with (d) narrow energy distribution (here: thermal emission) and (h) electron pulses with strong spectral broadening $(5.3 \mathrm{eV})$, there is only a slight degradation of the transverse beam properties (increase of minimum spot size from $2.2 \mathrm{~nm}$ to $3.5 \mathrm{~nm}$ ). For the measurements shown, the gun was operated in high coherence mode at $200 \mathrm{kV}(\mathrm{a}-\mathrm{c})$ and $120 \mathrm{kV}(\mathrm{d}-\mathrm{h})$ acceleration voltage, respectively, with spectral broadening induced by the near-field of a nanoscopic gold tip (cf. Fig. 4.8b and Ref. [34]).

generated by single-photon photoemission, in continuous or non space-charge broadened fs-operation, display comparable transverse beam properties to those thermally extracted from the emitter with identical electrostatic gun settings.

\subsection{Selected applications}

Low-emittance photoelectron beams, as demonstrated here, are ideally suited for ultrafast transmission electron microscopy with nanometer-scale spatial resolution (Fig. 4.6). Depending on the parameters of the condenser electron optics, the small transverse phase space area occupied by photoelectron bunches facilitates few-nanometer-scale electron foci, and collimated beams with $\mu \mathrm{m}$-scale transverse coherence lengths, respectively (cf. Table 4.1).

For all experiments, pre-alignment of the electron column in a continuous mode is possible by raising the temperature of the tip and entering the thermal emission regime of the Schottky emitter. Even after modification of the electron gun, high emission currents 


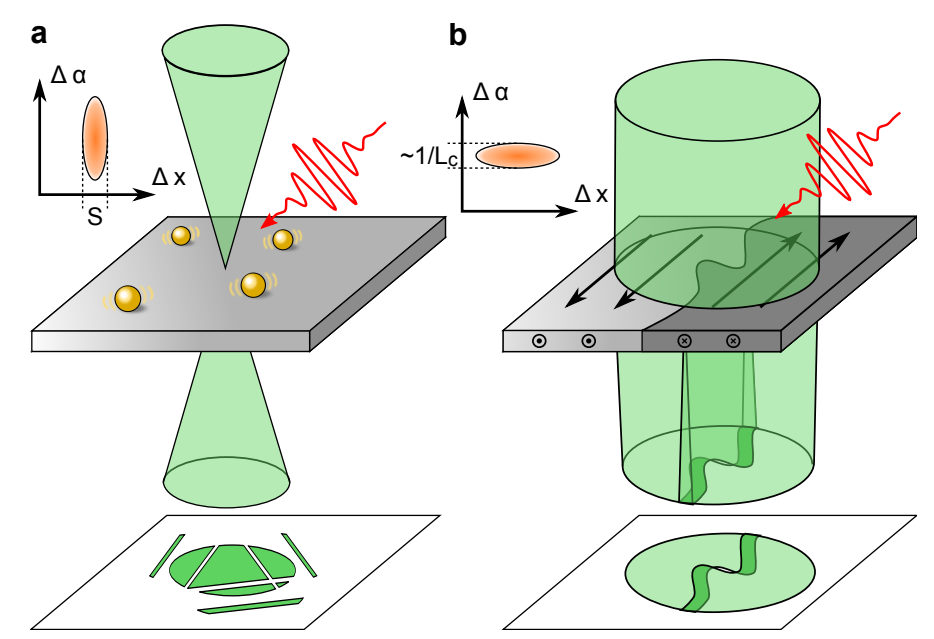

Figure 4.6: Applications of low-emittance electron pulses in ultrafast electron imaging. (a) Electron pulses focused to nanoscale spot sizes allow for local ultrafast probing, including ultrafast convergent beam electron diffraction (sketched here) and ultrafast electron energy loss spectroscopy. (b) For collimated low-emittance electron pulses, $\mu \mathrm{m}$-scale transverse coherence lengths are achievable, enabling phase-sensitive electron imaging techniques, such as ultrafast Lorentz microscopy (sketched here) as well as time-resolved variants of holographic techniques. Insets: Occupied area in the transverse phase space for a focussed and a collimated beam, respectively ( $S$ : focal spot size; $L_{\mathrm{C}}$ : transverse coherence length).

of several $\mu \mathrm{A}$ can be generated, thus enabling an in-situ characterization with high quality electron micrographs of the sample before and after time-resolved experiments, (e.g. highresolution TEM, cf. Fig. 4.7a). Switching between thermal and photoemission mode requires less than $1 \mathrm{~h}$.

Fig. 4.7 shows a set of examples demonstrating the present imaging, diffraction and spectroscopy capabilities of the Göttingen UTEM instrument in photoemission mode. With typical acquisition times of about 5-60s, electron micrographs are obtained which exhibit sufficient signal-to-noise ratio to map, for example, nanoparticles, bending contours (Fig. 4.7b) as well as nanoscale magnetic textures in Lorentz mode (Fig. 4.7c). Highquality electron diffraction patterns can be recorded both with parallel (Fig. $4.7 \mathrm{~d}$ and e) and convergent incident beams (Fig. 4.7f). We note that, despite working in the single- to few-electron per pulse regime, low intensity diffraction features arising from charge density waves can be clearly discerned. In addition, a high transverse coherence length of $1.2 \mu \mathrm{m}$ can be determined by scattering from a mesoscopic grating structure ( $463 \mathrm{~nm}$ spacing) (Fig. 4.7g). This will enable ultrafast electron holography (Fig. 4.7h) for the measurement of time-dependent electric and magnetic fields. Finally, the narrow spectral width of the 

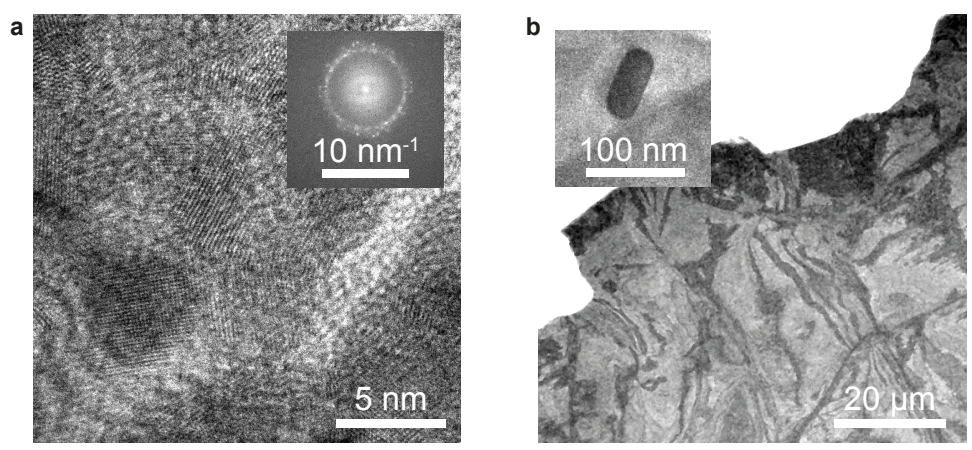

c
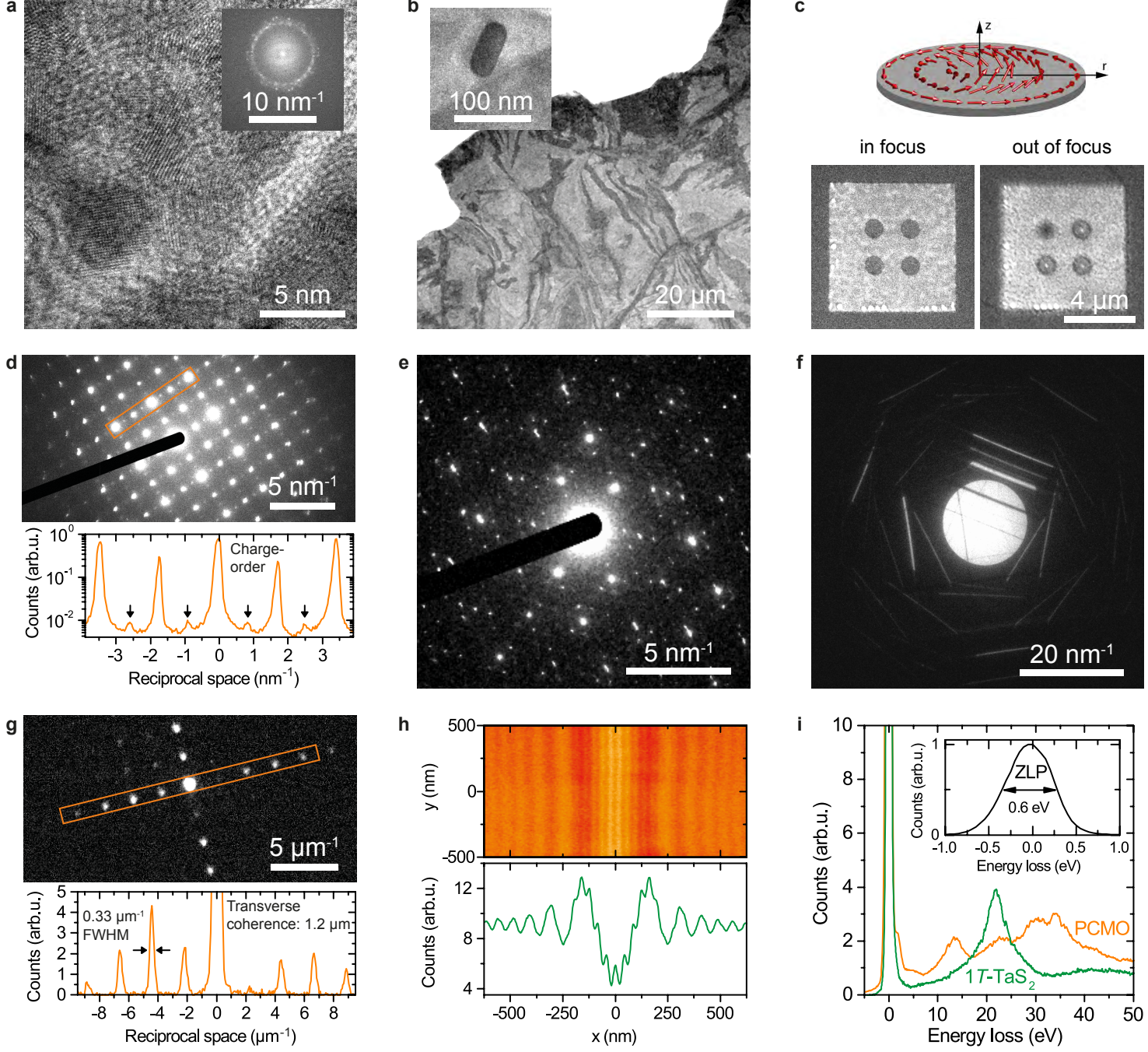

Figure 4.7: Exemplary experimental results achievable with the current status of the UTEM instrument. (a) High-resolution TEM (HRTEM) micrograph of Au/Pd particles on an amorphous carbon film. Visible lattice planes with $2-\AA$ spacing demonstrate the resolution capabilities of the modified instrument (here: using thermal electron emission). Inset: Fourier transform of a four times larger sample region. (b-i) Measurements acquired with photoelectron beams (typical acquisition times 5-60 s) and at an electron energy of $120 \mathrm{keV}$. (b) Bright-field image of an ultra-microtomed $50 \mathrm{~nm}$ thin sample of 1T-TaS2 showing bending contrast of the thin-film membrane. Close-up: drop-casted gold nanorod on the sample surface. (c) Lorentz imaging provides magnetic contrast in UTEM as demonstrated for permalloy islands on a silicon nitride support (see also Ref. [235]). The out-of-focus image reveals the existence of a magnetic vortex in each of the four islands (visible as black and white features, respectively, depending on vortex orientation). The magnetic structure of a single vortex is schematically depicted in the upper panel. (d) Diffraction pattern of the charge-ordered phase of an ion-polished PCMO $\left(\mathrm{Pr}_{0.7} \mathrm{Ca}_{0.3} \mathrm{MnO}_{3}\right)$ plan view sample. Weak superstructure spots are visible halfway between the lattice reflections. 
Figure 4.7 (Cont.): (e) Diffraction pattern of the nearly commensurate charge density wave (NC$\mathrm{CDW}$ ) phase of $1 \mathrm{~T}-\mathrm{TaS}_{2}$. The first-order NC-CDW diffraction spots are hexagonally arranged around structural reflections. (f) Convergent beam electron diffraction (CBED) pattern of an exfoliated $100 \mathrm{~nm}$ thick single-crystalline graphite flake. (g) High dispersion diffraction pattern of a $463 \mathrm{~nm}$ spaced grating replica, demonstrating 1.2- $\mu \mathrm{m}$ transverse coherence lengths. (h) Electron hologram obtained using a Möllenstedt biprism at a filament voltage $9 \mathrm{~V}$, emphasizing the photoelectron coherence properties achievable in the UTEM. (i) Electron energy loss spectra of $1 \mathrm{~T}-\mathrm{TaS}_{2}$ and PCMO. Inset: zero-loss peak (ZLP) with a FWHM of $0.6 \mathrm{eV}$.

electron beam of about $0.6 \mathrm{eV}$ allows for recording well-resolved electron energy loss spectra, which will facilitate the study of charge carrier dynamics and electronic structure in complex materials (Fig. 4.7i).

\subsection{Optical interactions with free-electron beams in the field-emitter UTEM}

Besides adding ultrafast temporal resolution to widely established electron microscopy techniques, the high coherence electron beams in UTEM also generate research directions completely outside the realm of conventional electron microscopy. A prominent class of new phenomena involve the interaction of the pulsed free-electron beam with intense optical fields [34, 90, 91, 93, 97, 223]. The exchange of energy and momentum between electromagnetic fields and free electrons provides multiple avenues of study, which include (i) the temporal characterization of ultrashort electron pulses (see Ch. 4.3.3) [223, 225], (ii) the nanoscale mapping of optical near-fields $[90,99]$, (iii) the active manipulation of freeelectron beams [34, 236], and (iv) the study of fundamental quantum optics phenomena [34, 90, 237].

In inelastic electron-light scattering (Fig. 4.8a), a beam of free electrons passes through the optical near-field of an illuminated nanostructure. The electrons exchange energy with the optical field in integer multiples of the incident photon energy, and consequently, the interaction transforms an initially narrow kinetic energy distribution into a symmetric spectral comb composed of a number of populated sidebands (see spectra in Fig. 4.8a). Physically, this process is closely related to electron energy loss (EEL) or cathodoluminescence (CL) at optical nanostructures [238-242]. All of these processes are facilitated by the near-field localization of optical excitations, which relax the requirement of conserving the total momentum in the electron and light fields alone by transferring excess momentum 
to a nanostructure. In EEL and CL, a swift electron passing a structure induces an optical polarization, with which it interacts [93]. Energy loss and cathodoluminescence then correspond to a spontaneous transition in the free-electron state, resulting in material absorption, the emission of far-field radiation or near-field excitations such as plasmons [93, 243]. In essence, the inelastic interaction described here may be viewed as the stimulated absorption and emission variants of these spontaneous processes.

This prompt interaction can be used in a variety of applications, and it was initially motivated by the desire to map optical near-fields in an approach termed photon-induced near-field electron microscopy (PINEM) [90, 91, 98]. In our UTEM, we implemented a scanning version of this technique (S-PINEM), in which a focused electron beam is scanned in the vicinity of an optically excited nanostructure, and an electron spectrum is recorded for every scan position (compare Ref. [34]), providing a quantitative measure of the optical near-field amplitude. Fig. 4.8b displays the fraction of inelastically scattered electrons at a metallic nanotip illuminated with polarizations perpendicular (right) and parallel (left) to the tip axis. Different optical near-field modes and a field-enhanced region at the apex of the conical structure (parallel illumination) are clearly visible in these images.

For increasing field strength, the spectra produced from this interaction exhibit a characteristic broadening and spectral oscillations in the individual sideband amplitudes (Fig. 4.8c). In Ref. [34], we experimentally demonstrated that these features are caused by multilevel Rabi oscillations in the free-electron states separated by the photon energy. In a spatial representation of the electron states, the interaction results in a sinusoidal phase modulation of the incident wave function [34, 91, 98]. As a result, dispersive propagation of the wavefunction after the interaction will cause a reshaping of the electron density subsequent to the interaction (Fig. 4.8d). Specifically, as shown in Ref. [34], the momentum modulation will cause a temporal focusing of the electron density into a train of attosecond pulses downstream in the electron microscope, at propagation distances in the one-to-few millimeter range (depending on the light frequency, the electron energy and the optical excitation strength). With a further nanostructure located in the region of the temporal focus (bottom in Fig. 4.8d), the arising attosecond pulse structure may be probed with a second, properly timed interaction. Representing a feasible means to generate attosecond electron pulses within the UTEM, this scheme will in the future allow for entirely new forms of optically phase-resolved electron microscopy and the study of electronic or structural dynamics with sub-femtosecond precision. 


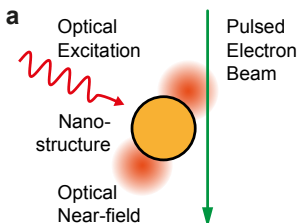

c

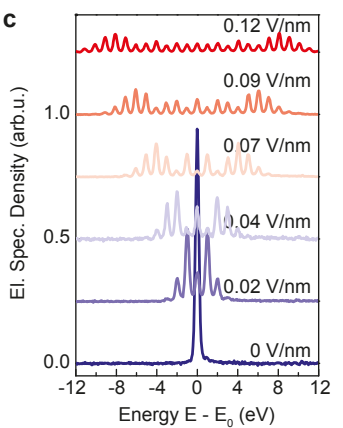

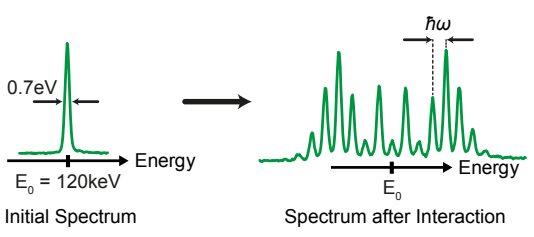

d

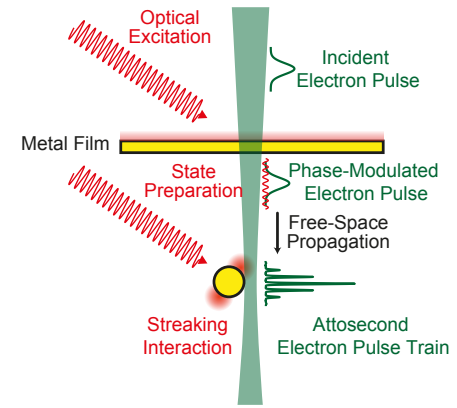

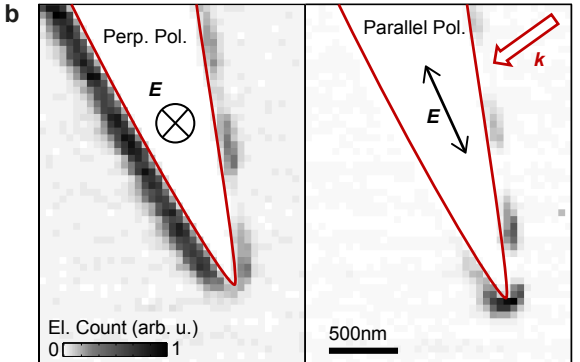

e

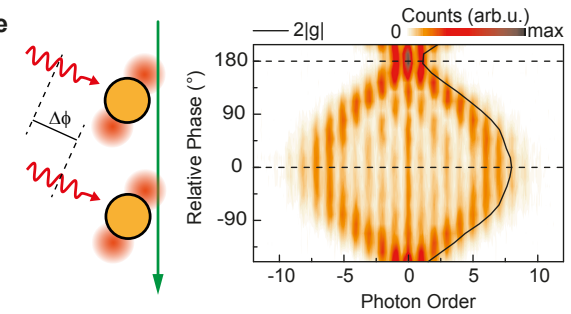

Figure 4.8: Applications of quantum coherent electron light interactions in optical near-fields within an ultrafast TEM. (a) The electron beam traversing an intense optical near-field develops into a comb of spectral sidebands, with (c) populations given by the optical field strength (assuming spatially and temporally homogeneous illumination). (b) Rasterscanning the electron beam allows for a quantitative mapping of the near-field distribution (S-PINEM) at a nanostructure (here: nanoscopic gold tip side-illuminated along the direction indicated by $\mathbf{k}$, with different optical polarizations and at $800 \mathrm{~nm}$ wavelength). (d) Dispersive broadening of the optically phase modulated single electron wavefunction leads to the formation of an attosecond train, with a temporal spacing given by the optical period. (e) Consecutive interactions with multiple near-fields enable Ramsey-type electron light interferometry, where the first interaction can be either cancelled out or enhanced depending on the relative excitation phase $((a-c)$ Adapted from Ref. [34], (e) adapted from Ref. [236]).

We recently applied the concept of multiple quantum coherent interactions with the same free-electron state in an experiment sketched in Fig. 4.8e (for details, see Ref. [236]). Here, two optical nanostructures are separated by several micrometers, i.e., at a distance for which no substantial electron density reshaping occurs. The electron beam sequentially interacts with two phase-locked optical near-fields, the relative phase of which can be precisely controlled. The color-coded image in Fig. 4.8e displays the resulting electron energy spectra for a variation of the relative phase. The final width of the energy spectrum, and thus the total impact of the interaction with the free-electron beam, is a strong function of this relative phase. This observation highlights the quantum coherent nature of these sequential interactions, in that the second action either cancels out or enhances the action of the first [236]. Such phase-controlled multiple interactions may form the basis of 
different variants of electron interferometry or time-domain holography, and - combined with optically excited materials inserted into the interferometer gap - may yield detailed information on nanoscale dephasing mechanisms on ultrashort timescales.

More generally, the demonstration of coherent and phase-sensitive optical near-field scattering opens up an exciting research path in the active quantum manipulation of electron wave functions. In particular, any electron wave packet of sufficient longitudinal and transverse coherence will directly carry the entire spatial and temporal amplitude and phase information of that optical near-field in a holographic fashion. Governing the further evolution of the electron probability density in space and time, such opticallyproduced holograms create far-reaching opportunities for coherent control schemes using free electrons, including the generation of specific transverse profiles and orbital angular momentum states $[17,52,54]$, or the arbitrary formation of temporal electron pulse structures.

\subsection{Conclusion and outlook}

In conclusion, we described the present status of our development of ultrafast transmission electron microscopy, using laser-triggered field emitters for the first time. We provided quantitative beam characterizations and presented exemplary imaging, diffraction and spectroscopy data recorded with this instrument.

Generally, nanoscopic cathodes offer superior performance over flat photocathode designs if nanoscale probing or high-sensitivity phase contrast are desired. Historically, many novel techniques in transmission electron microscopy, like sub-nm STEM probing and holography, were only enabled by the introduction of high-brightness field emitting electron sources. In the same way, the integration of tip-shaped photocathodes with an energy spread of less than $1 \mathrm{eV}$ and the peak brightness of a conventional Schottky emitter opens up new frontiers in electron microscopy and the study of nanoscale dynamics.

Nonetheless, further improvements in average source brightness are highly sought after for the further proliferation of the technique, and for widening its scope of applications. Alongside further development work on the actual source, an optimized electron gun geometry could increase the overall electron transmission from tip to sample, thus decreasing space-charge effects for a given final pulse charge, while minimizing aberrations and propagation-induced temporal pulse spread. Additional strategies will extend the practically accessible set of scientific problems with UTEM, such as devising intelligent 
drift correction schemes to allow for longer integration times. Moreover, the integration of aberration probe correction would enhance the acceptance of the TEM column and thus increase the total achievable pulse charge for a certain target specification in spatial electron beam parameters.

The very robust free-space laser excitation of the sample, as well as the long-term stability of our short-pulsed electron source, facilitate stable measurements on the nanoscale for periods exceeding $48 \mathrm{~h}$. This will enable the study of structural, electronic and spin dynamics with unprecedented spatial and temporal resolution. At present, ultrafast electron diffraction and spectroscopy from few-nanometer regions is well within the range of capabilities of the approach, as is phase contrast imaging and Lorentz microscopy. The generation and application of attosecond electron pulse trains will ultimately allow electron microscopy to enter the realm of attosecond science [244], adding nanoscopic spatial resolution to this exciting research field. With all these prospects, we believe that field-emitter-based UTEM technology will foster a greatly enhanced understanding of spatiotemporal dynamics, energy transport and relaxation processes on the atomic scale.

\section{Acknowledgments}

We acknowledge useful discussions and supporting interactions with Konrad Samwer, Christian Jooss, Michael Seibt, Cynthia Volkert, Markus Münzenberg, Max Gulde and Patrick Peretzki, as well as with Max Haider and Stefan Uhlemann (CEOS GmbH). For the preparation of samples, we are grateful to Kai Rossnagel (1T - $\left.\mathrm{TaS}_{2}\right)$, and Benedikt Iffland, Christian Jooss and Vladimir Roddatis (PCMO). Technical support by Karin Ahlborn and Matthias Hahn is gratefully acknowledged. We thank Felix Börrnert and Hannes Lichte for providing us with the bi-prism. We are also thankful for very productive collaborations with JEOL Ltd., JEOL (Germany) GmbH, and YPS Ltd., and we would like to note the particularly valuable technical support by Bernd Karoske (JEOL (Germany) GmbH).

This work was funded by the Deutsche Forschungsgemeinschaft (DFG) in the Collaborative Research Center "Atomic Scale Control of Energy Conversion" (DFG-SFB 1073, project A05) and in the Priority Program "Quantum Dynamics in Tailored Intense Fields" (DFG-SPP 1840). We gratefully acknowledge support by the Lower Saxony Ministry of Science and Culture and funding of the instrumentation by the DFG and VolkswagenStiftung. 



\title{
Chapter 5
}

\section{Quantum coherent optical phase modulation in an ultrafast transmission electron microscope}

\author{
A. Feist, K. E. Echternkamp, J. Schauss, S. V. Yalunin, S. Schäfer and C. Ropers \\ Nature 521, pp. 200-203, May 2015 \\ DOI: $10.1038 /$ nature14463
}

Coherent manipulation of quantum systems with light is expected to be a cornerstone of future information and communication technology, including quantum computation and cryptography [245]. The transfer of an optical phase onto a quantum wavefunction is a defining aspect of coherent interactions and forms the basis of quantum state preparation, synchronization and metrology. Light-phase-modulated electron states near atoms and molecules are essential for the techniques of attosecond science, including the generation of extreme-ultraviolet pulses and orbital tomography [244, 246]. In contrast, the quantum-coherent phase-modulation of energetic free-electron beams has not been demonstrated, although it promises direct access to ultrafast imaging and spectroscopy with tailored electron pulses on the attosecond scale. Here we demonstrate the coherent quantum state manipulation of free-electron populations in an electron microscope beam. We employ the interaction of ultrashort electron pulses with optical nearfields $[90,91,98,223,247,248]$ to induce Rabi oscillations in the populations of electron momentum states, observed as a function of the optical driving field. Excellent agreement with the scaling of an equal-Rabi multilevel quantum ladder is obtained [249], representing the observation of a light-driven 'quantum walk' [98] coherently reshaping electron density in momentum space [250]. We note that, after the interaction, the optically generated superposition of momentum states evolves into a train of attosecond electron pulses. 
Our results reveal the potential of quantum control for the precision structuring of electron densities, with possible applications ranging from ultrafast electron spectroscopy and microscopy to accelerator science and free-electron lasers.

\subsection{Introduction}

The interaction of propagating light with confined electrons in atoms, molecules and solids is omnipresent, but the opposite case-the coupling of free electrons to localized optical fields-is not a naturally occurring phenomenon. Nonetheless, in both cases, the principle of confinement allows for optical transitions in otherwise mismatched electron and photon dispersion relations [93]. Controlling free-electron propagation with low-frequency electromagnetic fields in resonator geometries is an integral aspect of accelerator science [251]. At optical frequencies, however, particular challenges arise from the requirements of very controlled electron beams and tailored nanostructure near-fields. Increasing efforts are currently devoted to optically drive electron trajectories on the nanoscale-for example, for applications in attosecond science and lightwave electronics [147, 231, 252-255].

Some of the elementary phenomena involved in coupling free electrons to light were described more than half a century ago: in the Kapitza-Dirac effect [256, 257], electrons are elastically scattered off a standing light wave, whereas the Smith-Purcell effect and its variants [247, 248, 258, 259] treat the inelastic interaction of free electrons with confined modes close to a grating. Recently, ultrafast electron microscopy schemes showed that the kinetic energy distribution of short electron pulses develops a series of photon sidebands after passage through an intense optical near-field [90, 91, 98]. This approach, termed photon-induced near-field electron microscopy (PINEM) [90], has been employed in the temporal characterization of ultrashort electron pulses (see Methods) and as a contrast mechanism in electron microscopy $[260,261]$. Beyond such advanced applications, the underlying interaction should allow for the preparation of coherent electronic superposition states and a phase-controlled harnessing of quantum coherence for the temporal shaping of electron bunches.

\subsection{Main part}

Here we report the coherent phase-modulation of free-electron states in a nano-optical field. We experimentally induce multilevel Rabi oscillations in the form of a quantum 
walk in momentum space, obtaining excellent agreement with theoretical predictions by García de Abajo et al. [98] and Park et al. [91] of this interaction. Moreover, we demonstrate theoretically that dispersive propagation transforms the optically modulated electron wavepacket into a train of attosecond peaks. In the experimental scenario displayed in Fig. 5.1, femtosecond electron pulses are generated by nonlinear photoemission from a nanoscale cathode $[150,151,174]$. After collimation and acceleration to an energy of $120 \mathrm{eV}$, the magnetic lens system of a transmission electron microscope focuses the electron pulses to a spot diameter of $15 \mathrm{~nm}$ in close vicinity to an optically excited conical gold tip. The localization of the nanostructure's near-field mediates the optical interaction with the free electrons. This leads to the creation of multiple spectral sidebands, each corresponding to the absorption/emission of an integer number of photons (spectrum in Fig. 5.1e) [90, 91, 98]. Detailed information about the interaction process is encoded in the number of populated sidebands and their individual amplitudes. For example, the maximum electron energy gain in the optical near field is a quantitative measure of the local transition amplitude, which can be imaged by raster scanning the electron focus (Fig. 5.1b).

Microscopically, the electron-light interaction studied here constitutes an optical phasemodulation of the electron wavefunction [91]. Expressed as a quantum mechanical multilevel system, electron energy levels spaced by the photon energy $\hbar \omega$ are coupled in the optical near-field (level diagram, Fig. 5.1d). Previous experiments studying this interaction found a partial reduction of the initial electronic state population and a spectral broadening with distributions gradually decaying towards large photon orders [90, 91, 223, 260, 261]. Such observations evidence transitions dominated by sequential multilevel excitation (processes of type I in Fig. 5.1d). However, it is assumed that the coupling process is coherent in nature $[91,98]$, which implies that quantum features arising from multipath interference (type II) should also be observable.

In order to identify such phenomena, we require an interaction of uniform strength with the entire electron ensemble in the pulse [98]. This scenario is achieved by using a spatially narrow probing beam and, in contrast to earlier works, an optical near-field excitation which has a uniform amplitude during the transit of the electron pulse envelope (see Methods).

Under these conditions, we find experimentally that the population of photon sidebands exhibits a pronounced oscillatory behaviour corresponding to multilevel Rabi oscillations, as demonstrated in Fig. 5.2 for electron spectra at a fixed position near the tip shaft. 
a
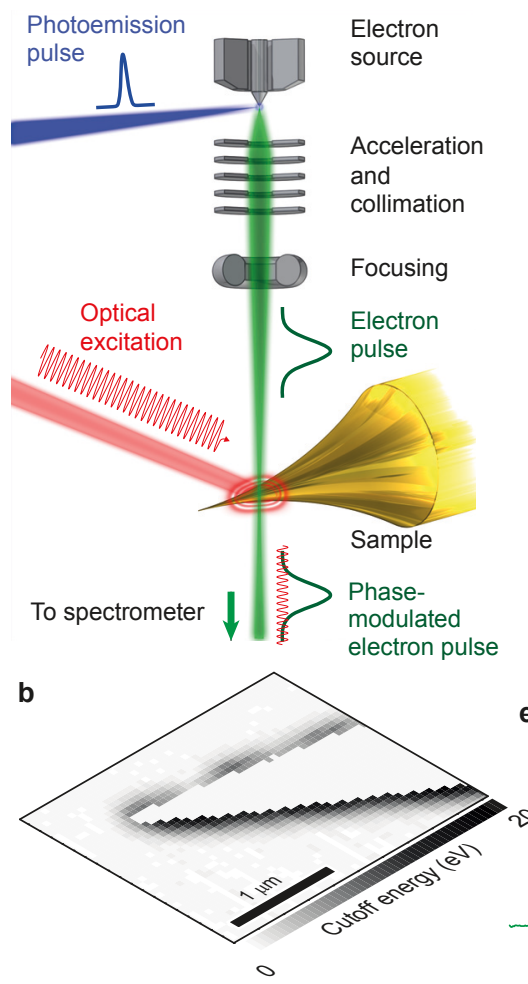

c
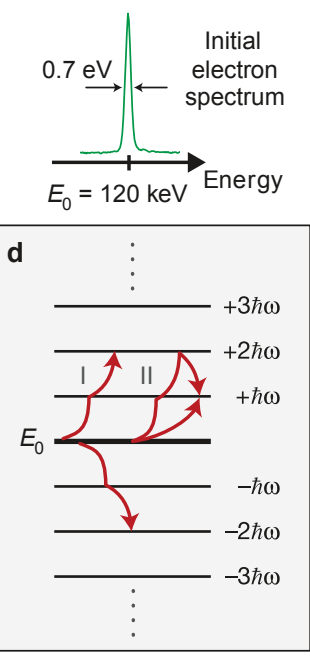

e Spectrum after interaction

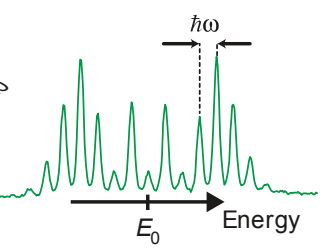

Figure 5.1: Schematic and principles of coherent inelastic electron scattering by optical near-fields. a, Experimental scheme. Ultrashort electron pulses generated by nanotip photoemission are accelerated and focused to a beam that interacts with the optical near-field of a nanostructure, phase-modulating the electron pulse and exchanging energy in integer multiples of the photon energy. $\mathbf{b}$, Raster-scanned image of the energy cutoff in the inelastic electron scattering spectra, representing the local transition amplitude (see text). c, Incident kinetic energy spectrum (fullwidth at half-maximum, $0.7 \mathrm{eV}$ ) centred at $E_{0}=120 \mathrm{keV}$. d, Energy level diagram of ladder states with spacing $\hbar \omega$ coupled to the initial state at $E_{0}$. Arrows indicate sequential multistate population transfer (type I) and interfering quantum paths (type II) leading to multilevel Rabi oscillations. e, Example of kinetic energy spectrum after the near-field interaction, exhibiting a spectral comb with multiple sidebands separated by the photon energy and modulated in occupation. 
A colour-coded map (Fig. 5.2a) displays the evolution of the interaction-induced kinetic energy distribution with growing incident field strength. With increasing driving field, we observe a linear spreading in the range of populated sidebands, together with strong oscillations in the central part of the spectra. Specifically, the experimental spectra exhibit a nearly complete extinction of the initial state occupation and its pronounced recurrence at incident fields of $0.023 \mathrm{~V} \mathrm{~nm}^{-1}$ (red line) and $0.040 \mathrm{~V} \mathrm{~nm}^{-1}$ (green line), respectively (Fig. 5.2c). Quantitative analysis of the field-dependent spectral evolution (Fig. 5.2b) shows the oscillations of the initial state population ('zero loss peak') and those of different electronic sidebands. These modulations directly evidence multilevel Rabi oscillations and thereby a quantum coherent manipulation of the respective level amplitudes, which, as a function of field strength, traces out the evolution of an elementary quantum walk [250].

Recently, near-field-induced free-electron transitions, as observed here, were theoretically treated by solving the time-dependent Schrödinger equation [91, 98] (compare our results to figure 2 in ref. [98], which depicts population oscillations simulated as a function of intensity). Yielding equivalent theoretical results, we present a compact description using raising and lowering operators acting on the electronic state $\left|E_{0}\right\rangle$ of the system at an initial energy $E_{0}$. As demonstrated in the Methods section, the action of the near-field can be described by a scattering matrix $S=\exp \left[g^{*} a-g a^{\dagger}\right]$ with a dimensionless near-field coupling constant $g$ proportional to the field strength and the transition matrix element. One may notice that $S$ takes the form of a displacement operator generating coherent states in the harmonic oscillator model. In a variation of this scenario, we use $a^{\dagger}$ and $a$ as commuting raising and lowering operators, connecting free-electron states separated by the photon energy, that is, $a^{\dagger}\left|E_{0}\right\rangle=\left|E_{0}+\hbar \omega\right\rangle$ and $a\left|E_{0}\right\rangle=\left|E_{0}-\hbar \omega\right\rangle$. The scattering matrix represents the Hamiltonian evolution of the system as a unitary operation on the initial electron wavefunction $\left|E_{0}\right\rangle$, which leaves the electron in a superposition of ladder states $\left|E_{0} \pm N \hbar \omega\right\rangle$, with $N$ a natural number.

In the limit of small optical driving fields, the scattering matrix is dominated by sequential multiphoton terms, for example, $\frac{1}{N !}\left(g a^{\dagger}\right)^{N}$, corresponding to type I transitions (Fig. 5.1d). The occurrence of interfering quantum paths at increased optical field strength (type II paths) becomes apparent by considering higher order terms in the Taylor expansion of $S$, such as $a^{\dagger}$ and $a^{\dagger} a^{\dagger} a$, which both facilitate the transition between the states $\left|E_{0}\right\rangle$ and $\left|E_{0}+\hbar \omega\right\rangle$, but each with a different phase factor in the final state.

Interestingly, because of the practically constant coupling matrix elements between adjacent levels (the 'equal Rabi' case [249]), the occupation probability of the $N$ th photon 
sideband can be described by a very simple analytical expression in the form of the $N$ thorder Bessel function of the first kind [91, 98], that is, $\left|\left\langle E_{0} \pm N \hbar \omega|S| E_{0}\right\rangle\right|^{2}=\left|J_{N}(2|g|)\right|^{2}$. In a spatial representation, these transitions arise from a sinusoidal phase modulation of the wavefunction traversing the optical near-field. Accordingly, such sideband populations are also commonly encountered in other physical systems using phase modulation, for example, in acousto-optics [262].

Comparing the experimental field-dependent electron populations with the analytical result (Fig. 5.2b), an excellent agreement is found both in the location and amplitudes of the respective occupation minima/maxima. The entire data set is described with a single Rabi phase $2|g|=F_{\text {inc }} \times 98 \mathrm{~V}^{-1}$ linearly increasing with the incident optical field strength $F_{\text {inc }}$, yielding a quantitative measure of the transition matrix element. As detailed in the Methods section, incomplete modulation of the Rabi oscillations at higher fields is caused by the finite spatial and temporal electron pulse widths within the optical near-field. Besides the predicted population oscillations, the characteristic low-field multiphoton limit of the electron-light interaction is also experimentally regained (slopes of $2 \mathrm{~N}$ in the field in a double logarithmic plot, see inset of Fig. 5.2b). Larger incident fields prominently transfer the electron distribution to the outer spectral lobes, creating a well-defined cutoff around $\left|E-E_{0}\right|=2|g| \hbar \omega$, equal to the maximum classical energy transfer. Thus, as in other instances of electrons driven by intense optical near-fields [147, 231, 255], the interaction energy is governed directly by the field amplitude instead of the ponderomotive energy.

This periodic phase (and correspondingly momentum) modulation of the electron wavefunction has important consequences for its subsequent evolution in free propagation. Generally, momentum modulation of classical states in particle accelerators is used for bunch compression [251], and an optical variant of this principle was recently proposed using ponderomotive forces acting on classical point particles [263]. However, the present conditions with electronic coherence times exceeding the optical period necessitate a quantum mechanical description of bunch reshaping. Figure 5.3a displays a few cycles of the simulated electron density in a periodically phase-modulated wavepacket as a function of the propagation distance and the arrival time at this distance relative to the centre of the pulse. Specifically, the phased superposition of momentum states reshapes into a high-contrast train of attosecond pulses at a well-defined distance downstream from the interaction region. For typical coupling constants achieved in the experiments, we obtain a temporal focusing into a train of pulses only about 80 as long, at a distance of $1.8 \mathrm{~mm}$ behind the sample. Further dispersion spreads the distribution corresponding to its 


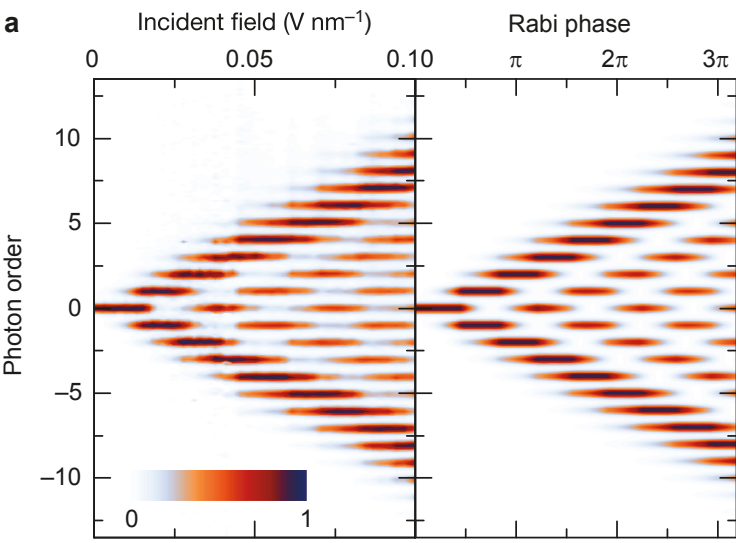

b
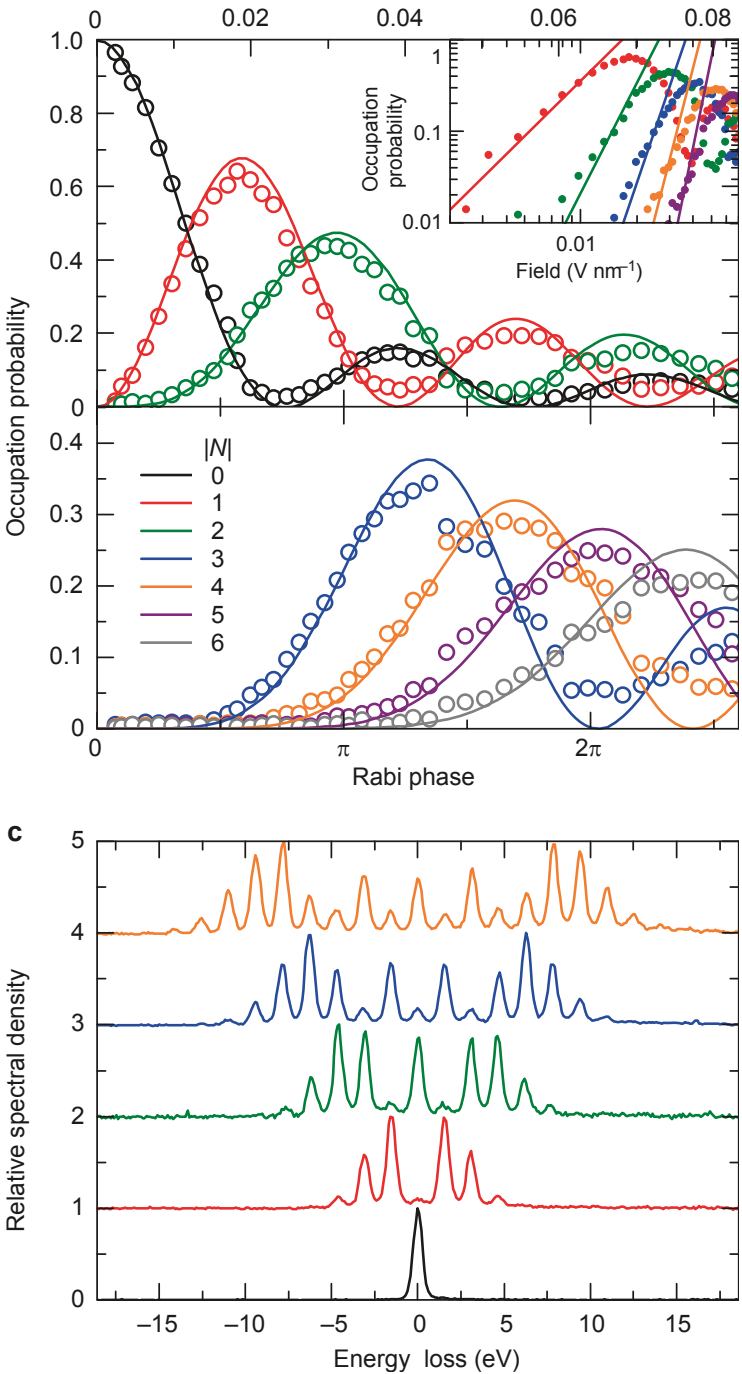

Figure 5.2: Quantum coherent manipulation of electron energy distributions. a, Experimental electron energy distributions as a function of the incident optical field strength (left) and theoretical prediction in terms of $N$ th-order Bessel functions (right). b, Occupation probabilities (open circles) of the $N$ th-order spectral sidebands shown in a, adding contributions from $\pm|N|$. Solid lines, $N$ thorder Bessel functions. Inset, doublelogarithmic plot of the sideband populations near onset. Solid lines with slope $2 \mathrm{~N}$ are shown for comparison. c, Electron energy spectra at incident optical fields of $0,0.023,0.040$, 0.053 and $0.068 \mathrm{~V} / \mathrm{nm}$ (bottom to top). Spectra shown in a and $\mathbf{c}$ are normalized to their respective maxima. 
momentum content, with the possibility of revivals. Note that a spatial optical equivalent of this generation of attosecond spikes is given by Fresnel diffraction at a sinusoidal phase grating into a near-field fringe pattern [264], and also that an early theoretical scheme for subfemtosecond optical pulse generation relied on frequency modulation and subsequent reshaping [265].

The physical origin of this electron pulse compression can be illustrated using a phase space representation of the quantum state in the form of a Wigner function. This function is a quantum mechanical analogue of a phase space density, which, however, can also take negative values for non-classical states [104]. Figure 5.3b displays the Wigner function of one period of a propagated state at the temporal focus and for a typical momentum distribution (projection in Fig. 5.3c). In this plane, free propagation of the initially sinusoidal momentum modulation has sheared the phase space distribution to a situation where a highly localized projection onto the position axis, that is, arrival time, is formed (Fig. 5.3d). In fact, the generation of this attosecond electron pulse train is very robust with respect to variations of the specific temporal and energetic structure of the initial electron pulse (see Methods). The practically usable focal distances (a few millimetres) render this scheme directly applicable in electron microscopy or spectroscopy studies with attosecond precision, a domain at present only accessible by attosecond light pulses [244]. Specifically, the temporal electron comb will enable the phase-resolved investigation of coherent sample excitations, thus tracing structural or electronic changes carrying optical phase information.

\subsection{Conclusion}

In conclusion, we have demonstrated the quantum coherent manipulation of free-electron wavefunctions by their interaction with nanoconfined light fields, observing near-perfect correspondence to the behaviour of a multilevel model Hamiltonian. Thinking beyond a single-variable state control, near-field interactions are expected to cause entanglement of longitudinal and transverse momentum components, and moreover, Coulomb interactions in a beam crossover will result in correlations between multiple electrons. Both features may be crucial for employing free electrons in quantum information technology [245]. Perhaps surprisingly, the generation of an attosecond electron train is a direct and natural consequence of this optical phase-modulation. We anticipate various applications of this concept in imaging and spectroscopy-for example, in the phase-resolved detection of 

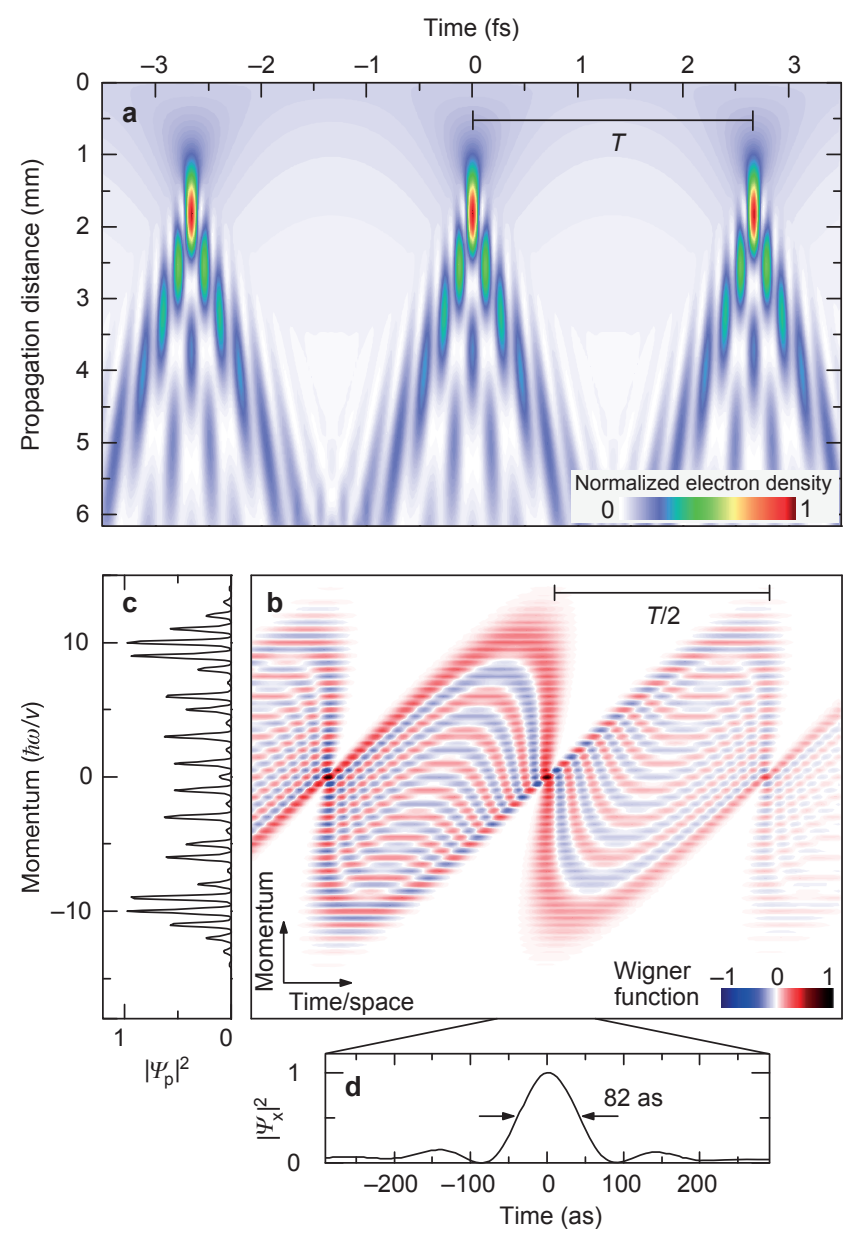

Figure 5.3: Formation of an attosecond electron pulse train. a, Development of a periodically modulated electron pulse structure (normalized electron density is colour coded) as a function of the propagation distance after the near-field interaction (numerical simulation for $|g|=5.7$ ). Free propagation causes a temporal focusing into a train of attosecond spikes (red) with a period of $T=2.55 \mathrm{fs}$ (optical period). $\mathbf{b}$, Phase space (Wigner) representation of one period of the lightmodulated electron quantum state at the temporal focus position (propagation distance of $1.8 \mathrm{~mm}$ in a). Note that time and space variables for the swift electron pulse can be used equivalently via $x=v t$ ( $v$ is mean electron velocity). $\mathbf{c}$, Momentum projection of Wigner function exhibiting spectral modulations as observed in the experiments, displayed in units of transferred momentum quanta (average momentum subtracted). d, Central part of spatial projection, expressed in terms of electron arrival time in laboratory frame. A peak with a duration of only 82 as (FWHM) is produced. 
coherent, resonantly driven polarizations in solid state materials-thus opening up the study of attosecond phenomena in electron microscopy.

\subsection{Methods}

\subsubsection{Ultrafast TEM and experimental setup}

We have recently constructed an ultrafast TEM (UTEM) to enable a variety of laserpump/electron-probe imaging schemes with high spatial resolution. The microscope is based on a commercial Schottky field emission TEM (JEOL JEM-2100F), which we modified to allow for both optical sample excitation and laser-driven electron pulse generation in the gun, as shown in Extended Data Fig. 5.4. In contrast to previous implementations of time-resolved TEM, our instrument features a nanotip photocathode as the source of ultrashort electron pulses. Compared to planar emitters, such needle cathodes provide reduced electron beam emittance, which is particularly useful for nanoscale probing and spectroscopy. In the present experiments, we employ electron pulses with a repetition rate of $250 \mathrm{kHz}$, which are induced via two-photon photoemission by irradiating the apex of a tungsten field emission cathode (apex radius of curvature of about $120 \mathrm{~nm}$ ) with ultrashort laser pulses ( $400 \mathrm{~nm}$ central wavelength, $50 \mathrm{fs}$ pulse duration, $4.1 \mathrm{~nJ}$ pulse energy, $25 \mathrm{GW} \mathrm{cm}^{-2}$ incident peak intensity). The emitter tip is operated at room temperature and with an electrostatic extraction field of $0.1 \mathrm{~V} \mathrm{~nm}^{-1}$.

At an electron energy of $120 \mathrm{keV}$, the beam is focused to a spot diameter of about $15 \mathrm{~nm}$, with a typical electron pulse duration of 700-900 fs (full-width at half maximum, FWHM) at the position of the sample (characterized by electron-laser cross-correlation, see below). This pulse duration is at present governed by velocity dispersion of electrons with different initial kinetic energies after photoemission, which, however, is not a limitation for the measurements described here. While pulses may contain many electrons, all experiments reported here were acquired with less than one electron per pulse at the sample position (single-electron mode), thus avoiding potential space charge influences.

The laser pulse exciting the optical near-field (central wavelength of $800 \mathrm{~nm}$, pulse energy of up to $60 \mathrm{~nJ}$ ) is stretched by dispersion in glass to a pulse duration of $3.4 \mathrm{ps}$ and focused to a spot size of $\sim 50 \mu \mathrm{m}$, yielding peak intensities of $1.33 \mathrm{GW} \mathrm{cm}^{-2}$ at the largest applied fluence. The excitation with a laser pulse of a duration much longer than that of the electron pulse allows for the observation of coherent population oscillations (see section 


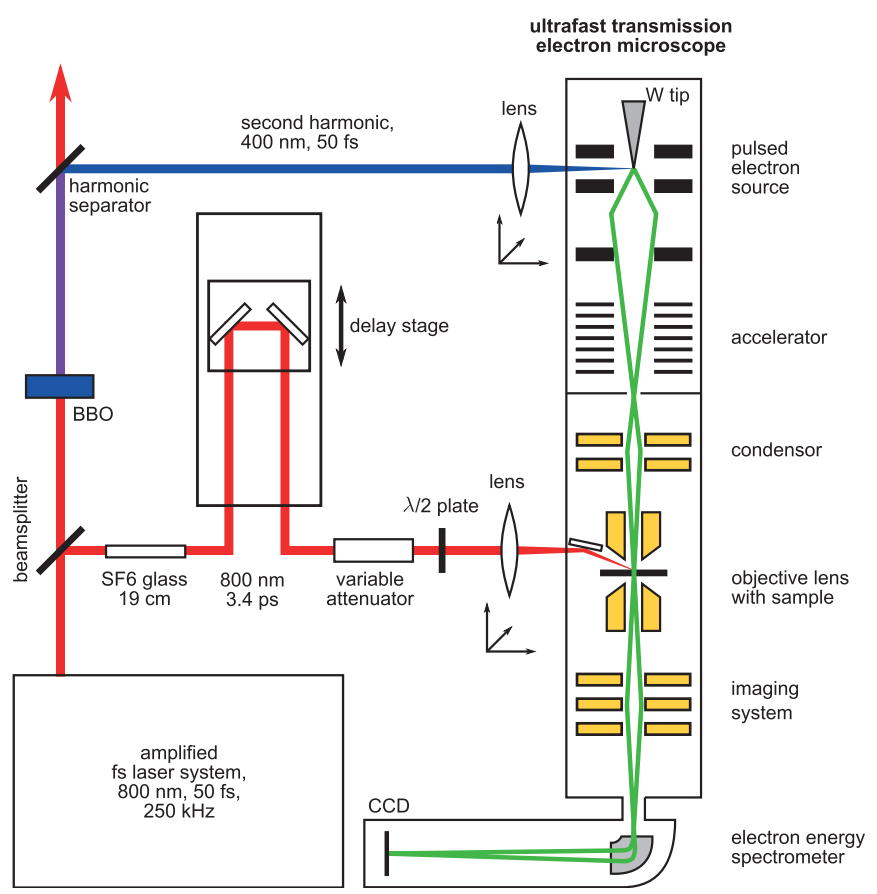

Figure 5.4: Experimental setup. Pulses from an amplified femtosecond (fs) laser system, at bottom left, are split into two optical beams. One of them is frequency-doubled in a $\beta$-barium borate (BBO) crystal and, after separation from the fundamental beam, focused (lens with numerical aperture $0.015,50 \mathrm{~cm}$ focal length) onto the tungsten needle emitter (W tip) for the generation of electron probe pulses. The second beam (pump beam) is temporally stretched, attenuated and focused (lens with numerical aperture $0.014,20 \mathrm{~cm}$ focal length) onto the sample within the TEM (angle of incidence, $55^{\circ}$ ). Relative timing between the electron probe and laser pump pulse is controlled by an optical delay stage. Optically-induced changes of the population of electron momentum states are recorded with an electron energy spectrometer. See Methods for details.

'Numerical calculations' below).

After interaction with the optical near-field, the electron pulse is imaged (magnification of 25,000) into an electron energy-loss spectrometer (EELS) to analyse its kinetic energy distribution (spectrometer entrance aperture of $3 \mathrm{~mm}$, energy dispersion of $0.05 \mathrm{eV}$ per detector channel).

Modulated electron spectra are observed at any electron probe position within the optical near-field. However, the quality of the spectral modulation and full extinction of individual orders crucially depends on the transverse homogeneity of the near-field on the scale of the electron beam diameter (see section 'Numerical calculations' below). For a nanoscopic tip, the optical near-fields are only slowly varying along its shaft. Therefore, we chose a position of the electron focus several micrometres away from the tip apex (see Extended 


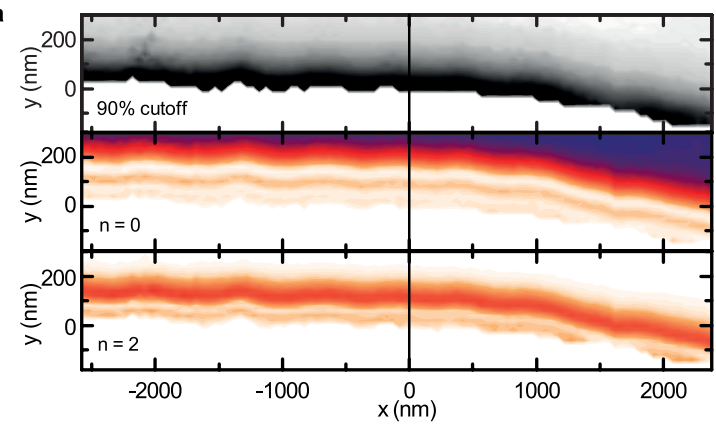

b

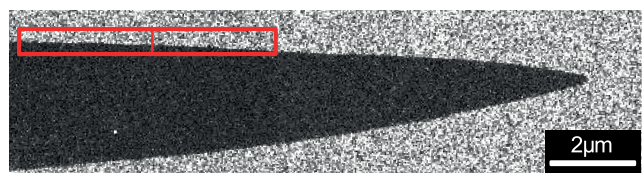

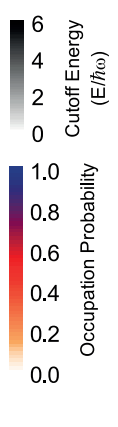

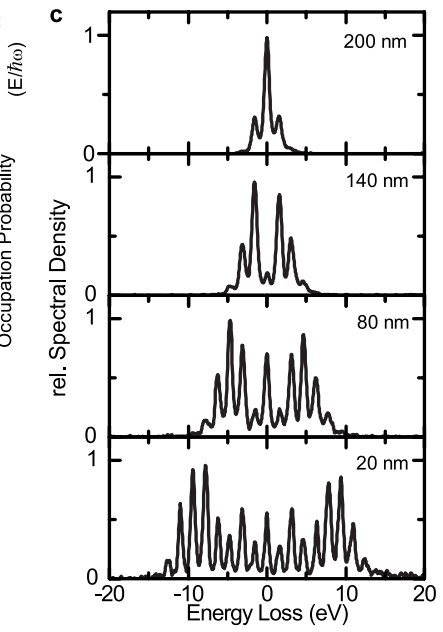

Figure 5.5: Spatial characterization of near-field scattering. a, Raster scan of the optically-induced electron energy gain and loss probability, characterized by the spectral cutoff (top panel) and the sideband populations of the zero loss peak (middle) and the second photon order (bottom). The field-dependent electron energy spectra shown in Fig. 5.2 of the main text were recorded at an $x$ position indicated by the black line at the tip surface. A slow sample drift results in a scanning artefact in the $y$ direction (jagged edge of the tip). For the results reported in the main text, a drift correction in the $y$ direction was applied (see Methods section 'Data analysis and drift correction'). b, TEM image of gold tip. Red rectangle, scanning area displayed in a. c, Electron energy-loss spectra recorded along $x=0$ with varying distance from the tip surface.

Data Fig. 5.5).

\subsubsection{Temporal characterization of electron pulses}

In UTEM, the duration of the electron pulses is governed mainly by Coulomb repulsion and dispersive broadening within the electron gun and subsequent electron optics. The resulting electron pulse structure in the sample plane can be quantitatively characterized employing inelastic near-field electron scattering [99, 100, 225], as discussed in the main text. However, to this end, and in contrast to the experimental conditions described in the previous section, laser pulses of a duration (here $50 \mathrm{fs}$ ) much shorter than the electron pulse duration should be used in an electron/near-field cross-correlation [225].

Extended Data Fig. 5.6a displays electron energy spectra as a function of the temporal delay between the optical excitation and the electron pulse arrival, subtracting the electron spectrum in the absence of near-field excitation. Therefore, the central blue feature corresponds to the reduction of the zero-loss peak due to scattering of electrons into multiple photon sidebands (red stripes). The width of these features (Extended Data Fig. 

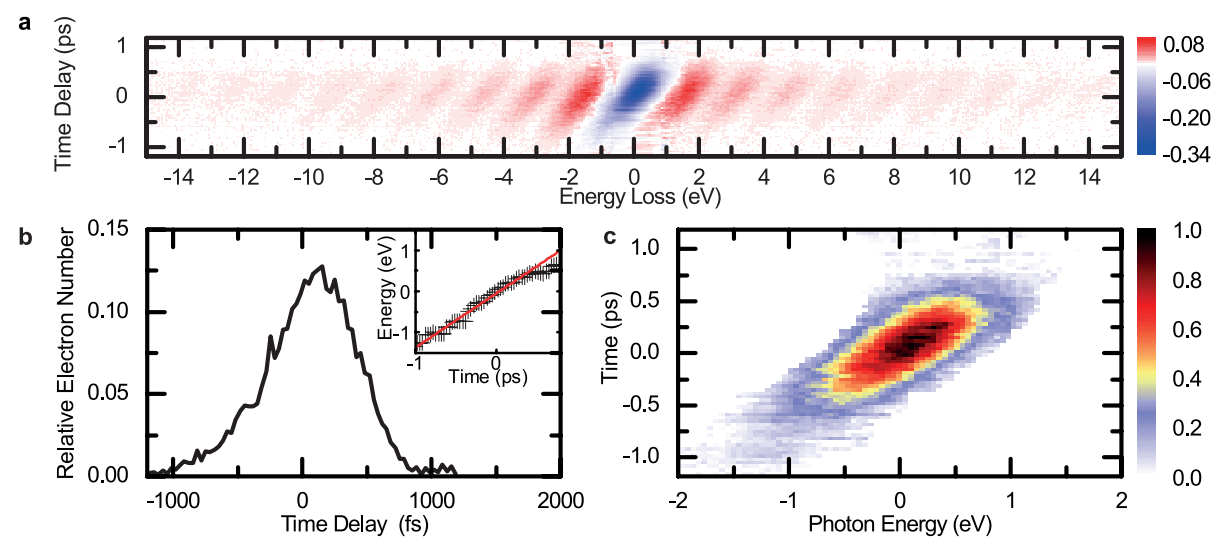

Figure 5.6: Pulse characterization by electron-photon cross-correlation. a, Differential electron energy-loss spectra as function of time delay (zero loss peak of width $1.3 \mathrm{eV}$ subtracted; the colour scale shows the relative change of spectral density). b. Relative total scattering amplitude as function of time delay (inset, relative shift of photon sidebands with respect to zero loss peak). c, Energy- and time-resolved structure of the electron pulse (the colour scale shows the normalized electron density).

5.6b) and their tilt in the energy-time diagram (inset) provide measures of the electron pulse duration and chirp, respectively. Here, we obtain a cross-correlation with a FWHM of $800 \mathrm{fs}$ (standard deviation of $340 \mathrm{fs}$ ) and a chirp of $\sim 760 \mathrm{fs} \mathrm{eV}^{-1}$ for an initial energy spread of $1.3 \mathrm{eV}$. In the experiments presented in the main text, electron pulses with a narrower energy spread of $0.7 \mathrm{eV}$ were achieved. For the much shorter near-field excitation employed here, the scattering signal is linear in the momentary density of electrons within the pulse. Therefore, we can extract an energy-time representation of the electron pulse by superimposing all tilted sidebands (Extended Data Fig. 5.6c). Finally, we emphasize that temporally stretching the near-field excitation to more than $3 \mathrm{ps}$ in the experiments ensures that it lasts much longer than the electron pulse duration and, therefore, provides for a nearly homogeneous scattering amplitude throughout the electron pulse.

\subsubsection{Data analysis and drift correction}

In order to obtain high quality electron spectra at a fixed sample location for varying driving field strengths, it has to be ensured that the laser-induced sample displacement, for example, by thermal expansion, is compensated for. This was achieved by first characterizing the fluence-dependent sample shift in imaging mode (observed up to $150 \mathrm{~nm}$ ) and by automated electron beam repositioning between experimental runs. In addition, slow residual drifts (up to $20 \mathrm{~nm}$, see Extended Data Fig. 5.5a) were corrected for by continuous line scans 


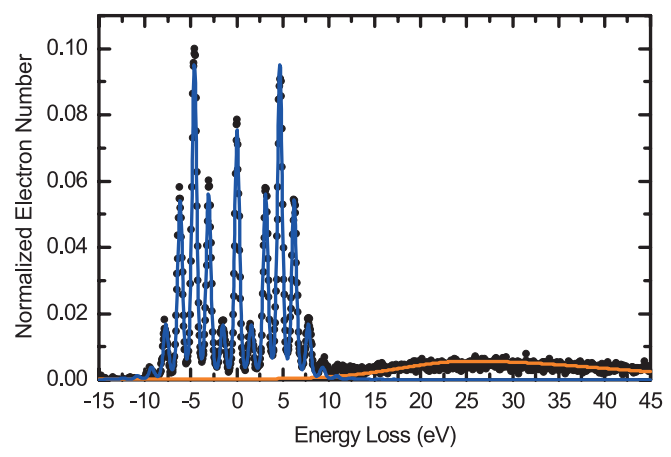

Figure 5.7: Evaluation of sideband populations. Example of electron energy spectrum (black dots) showing a number of photon sidebands and a weak low-loss plasmon contribution. Lines show fitted function used to extract sideband populations (blue) and the plasmon band (orange).

perpendicular to the gold surface and using the strength of the bulk plasmon band to identify the beam-surface distance. In the recorded spectra, energy losses due to bulk plasmon excitation generate a weak and spectrally broad band at energies above $15 \mathrm{eV}$ (ref. [92]), which is only present when the electron beam is placed in close proximity to the tip so that the outer tail of the electron focal spot grazes the tip surface. The plasmon contribution is well-separated from the main spectral features and can be easily subtracted from the spectra. Specifically, we identify the sideband populations by adopting a global fit function containing pseudo-Voigt profiles $V_{\mathrm{P}}(E)$ for the zero-loss peak and all photon sidebands (using symmetric amplitudes in $\pm|N|$ ). The plasmon peak at a loss energy $E_{\mathrm{pl}}$ was described by an asymmetric Gaussian $G_{\mathrm{pl}}(E)$ :

$$
P(E)=G_{\mathrm{pl}}\left(E-E_{\mathrm{pl}}\right)+\sum_{N=-\infty}^{\infty} a_{|N|} V_{\mathrm{P}}(E+N \hbar \omega)
$$

Extended Data Fig. 5.7 shows a typical electron spectrum together with the fitted function. Note that an energetic shift of the sideband comb relative to the zero-loss peak due to electron chirp is absent in the case of a long excitation pulse relative to the electron pulse duration and does not have to be included in the fit. An evaluation of the strength of the plasmon band as a function of beam-surface distance allows for a positioning accuracy of $\pm 5 \mathrm{~nm}$. 


\subsubsection{Materials}

The nanostructure employed in this work was prepared from a thin gold wire (diameter $250 \mu \mathrm{m})$ which was subjected to thermal annealing in vacuum $\left(800^{\circ} \mathrm{C}, 12 \mathrm{~h}\right)$ to increase crystallinity and reduce surface roughness[266]. A sharp tip (100 nm apex radius) was formed by electrochemical etching in aqueous hydrochloric acid (37\%) [267]. Afterwards, the conical part of the tip (length $\sim 50 \mu \mathrm{m}$ ) was cut by focused ion beam milling, transferred to a silicon frame and attached by ionbeam deposited platinum.

\subsubsection{Quantum description using ladder operators}

The interaction of electrons traversing an optical near-field has been theoretically treated several times in the past, usually by either direct integration of the time-dependent Schrödinger equation [91] or using a Green's function formalism [98, 268]. The relation of this stimulated process to the spontaneous mechanisms observed by electron energy-loss spectroscopy and cathodoluminescence is discussed in ref. [268]. Furthermore, it was also shown that a non-relativistic approach is sufficient as long as the relativistically correct electron dispersion (velocity as a function of energy) is used in the final result [222]. Here, we present an alternative derivation of inelastic near-field scattering probabilities using ladder operators, which allows for a succinct description.

The raising and lowering operators Electrons in a time-harmonic electromagnetic field can experience energy loss or gain in multiples of the photon energy $\hbar \omega$, where $\omega$ is the frequency of the field. This allows us to treat the problem as a multilevel quantum system. Within the Schrödinger picture, the free-electron Hamiltonian $H_{0}$ does not depend on time, while the wavefunction $|\psi(t)\rangle$ of the electron is time-dependent. Thus, the total Hamiltonian for the interaction with the electromagnetic field in the velocity gauge is

$$
H=H_{0}+\frac{e}{m} p A
$$

where $A$ is the space- and time-dependent vector potential, and $p, e$ and $m$ are the electron momentum, charge and mass, respectively. For a time-harmonic vector potential, a natural basis set is composed of plane wave states $|N\rangle$ offset from the initial energy $E_{0}$ by an integer multiple $N$ of the photon energy, where each state $|N\rangle$ is an eigenstate of the unperturbed Hamiltonian $H_{0}: H_{0}|N\rangle=\left(E_{0}+N \hbar \omega\right)|N\rangle$. Thus, $|0\rangle$ is the initial state, and 
$|N\rangle$ corresponds to the state with $|N|$ absorbed/emitted quanta. The time-harmonic interaction Hamiltonian causes transitions between these basis states. In particular, the matrix elements between adjacent states of the form $\left\langle N+1\left|\frac{e}{m} p A\right| N\right\rangle$ will lead to considerable transition probabilities. In contrast, the coupling between states separated by more than one photon energy causes transition amplitudes rapidly oscillating in time (at multiple frequencies of $\omega$ ), which prevents direct multiphoton transitions. (Note that multiphoton transitions will become possible by multiple actions of the field.)

In order to compute the coupling between neighbouring states, let us consider for simplicity a one-dimensional model with the time-harmonic vector potential $A=F(z) \sin (\omega t) / \omega$, where $F(z)$ is the spatial distribution of the electric field amplitude. To obtain the matrix elements $\left\langle N+1\left|\frac{e}{m} p A\right| N\right\rangle$, we use $|N\rangle$ in a plane wave form $L^{-\frac{1}{2}} \exp \left(i k_{N} z\right)$ in a finite spatial interval of length $L$, where $\hbar k_{N}$ is the electron momentum. In this representation, the matrix elements can be readily computed, for instance

$$
\left\langle N+1\left|\frac{e}{m} p A\right| N\right\rangle=\frac{2 \hbar v_{N} g}{L} \sin (\omega t), g=\frac{e}{2 \hbar \omega} \int_{-L / 2}^{L / 2} F(z) \exp (-i \Delta k z) \mathrm{d} z
$$

where $v_{N}$ is the electron velocity in the state $|N\rangle$, and $\Delta k \approx \omega / v_{N}$ is the electron momentum change (divided by $\hbar$ ). The dimensionless coupling constant $g$ expressed in terms of a Fourier amplitude in equation (5.3) was introduced in a similar form as used in ref. [91]. Physically, $g$ describes the momentum component in the near-field distribution which allows for total energy and momentum conservation in the transition, that is, it represents the momentum change of an electron undergoing an energy transition of $\hbar \omega$. Regarding the integration limits in equation (5.3), at present, it is only important that the interval length $L$ is larger than the extension of the near-field, as $L$ will cancel out in the final result. It should be noted that for an initial energy much higher than the maximum number of absorbed or emitted photons, $E_{0} \gg|N| \hbar \omega$, the coupling matrix elements in equation (5.3) become practically independent of $N$, as does the velocity $v \approx v_{N}$. The presence of a single and universal coupling constant renders the present quantum system a nearly perfect example of an equal Rabi multilevel system [249]. The transitions in this system can be concisely described by introducing the raising and lowering operators $a^{\dagger}$ and $a$, respectively, as

$$
|N+1\rangle=a^{\dagger}|N\rangle, \quad|N-1\rangle=a|N\rangle
$$


Note that, in contrast to the commonly employed ladder operators of a harmonic oscillator (which has a coupling constant scaling with $\sqrt{N}$ ), it follows from equation (5.4) that $a$ and $a^{\dagger}$ commute: $a a^{\dagger}=a^{\dagger} a$. The essential parts of the interaction Hamiltonian then take a bi-diagonal form, which can be represented in the raising and lowering operators

$$
\frac{e}{m} p A=\frac{2 \hbar v}{L}\left(g^{*} a+g a^{\dagger}\right) \sin (\omega t)+O\left(a^{n}, a^{\dagger n} ; n \geq 2\right)
$$

The higher order contributions can be neglected in the following, as they lead to negligible transition probabilities (see below), and terms on the main diagonal are absent because the spatial integral over the near-field distribution $F(z)$ (the case of $\Delta k=0$ ) vanishes.

The S-matrix To obtain transition probabilities for electrons after passage through the near-field, it is convenient to switch to the interaction picture. Here, the lowering and raising operators become time-dependent: $a(t), a^{\dagger}(t)$. In our case, they can be easily expressed in terms of $a$ and $a^{\dagger}$ by the transformation

$$
a(t)=\exp (-i \omega t) a, \quad a^{\dagger}(t)=\exp (i \omega t) a^{\dagger}
$$

and the interaction Hamiltonian turns into

$$
H_{\text {int }}(t)=\frac{2 \hbar v}{L} \sin (\omega t)\left[\exp (-i \omega t) g^{*} a+\exp (i \omega t) g a^{\dagger}\right]
$$

where $a$ and $a^{\dagger}$ denote the time-independent lowering and raising operators (see equation (5.4)). The temporal evolution of the quantum system can be treated in terms of a scattering matrix $S$, defined as a unitary transformation connecting asymptotic particle states $|\psi(\infty)\rangle=S|\psi(-\infty)\rangle$ before and after the interaction (for the time-dependence of the electron wavefunction during near-field transit, see ref. [98]). This unitary operator $S$ is given by the time-ordered exponent

$$
S=T \exp \left(-\frac{1}{\hbar} \int_{-\infty}^{\infty} H_{\mathrm{int}}(t) \mathrm{d} t\right)
$$

In the present case, the time-ordering $T$ can be omitted because $a(t)$ and $a^{\dagger}(t)$ commute. With the choice of a finite support $L$ of the basis states, the range of integration should in principle be limited to $\int_{-L / 2 v}^{L / 2 v}$, which will cancel out the ratio $v / L$ appearing in equation 
(5.7) for the time-independent contributions. The terms oscillating at higher frequencies $\left(\int \exp (2 i \omega t) \mathrm{d} t\right.$ in equation (5.7) and higher order contributions from equation (5.5)) vanish in the limit of large $L$. This case of large $L(L>v / \omega)$ corresponds to the experimental situation, in which the momentum states are well-resolved with respect to their energy difference $\hbar \omega$, and therefore, the passage to infinity can be carried out without loss of generality:

$$
\frac{1}{\hbar} \int_{-\infty}^{\infty} H_{\mathrm{int}}(t) \mathrm{d} t=\frac{g^{*} a-g a^{\dagger}}{i}
$$

Thus, the $S$-matrix in the interaction picture can be finally written as

$$
S=\mathrm{e}^{g a^{\dagger}-g^{*} a}
$$

and interestingly, the scattering matrix takes on the form of a displacement operator [104].

The transition probabilities Using the $S$-matrix, we can compute the probabilities of the transitions $|0\rangle \rightarrow|N\rangle$, given by $P_{N}=|\langle N|S| 0\rangle|^{2}$. For this purpose, we first split the matrix exponent in equation 5.10 into a product of two exponents, $\exp \left(g a^{\dagger}-g^{*} a\right)=$ $\exp \left(g a^{\dagger}\right) \exp \left(-g^{*} a\right)$. This separation is of course possible because $a$ and $a^{\dagger}$ commute. Expanding the exponential operators in a Taylor series, we find

$$
\exp \left(g a^{\dagger}\right)|0\rangle=\sum_{m=0}^{\infty} \frac{g^{m}}{m !}\left(a^{\dagger}\right)^{m}|0\rangle=\sum_{m=0}^{\infty} \frac{g^{m}}{m !}|m\rangle
$$

and analogously

$$
\langle N| \exp \left(-g^{*} a\right)=\sum_{n=0}^{\infty} \frac{\left(-g^{*}\right)^{n}}{n !}\langle n| a^{n}=\sum_{n=0}^{\infty} \frac{\left(-g^{*}\right)^{n}}{n !}\langle N+n|
$$

Using equation 5.10 and the orthogonality relation $\langle N+n \mid m\rangle=\delta_{N+n, m}$, we obtain

$$
\langle N|S| 0\rangle=\sum_{n=0}^{\infty} \frac{\left(-g^{*}\right)^{n} g^{n+N}}{n !(n+N) !}=g^{N} \sum_{n=0}^{\infty} \frac{\left(-|g|^{2}\right)^{n}}{n !(n+N) !}
$$


Comparing this result with the following series expansion for the Bessel function of the first kind

$$
J_{N}(z)=(z / 2)^{N} \sum_{n=0}^{\infty} \frac{\left(-z^{2} / 4\right)^{n}}{n !(n+N) !}
$$

we finally obtain

$$
P_{N}=J_{N}(2|g|)^{2}
$$

Therefore, the probability of energy gain or loss is given in the form of Bessel functions of different order [91].

Propagation after interaction and Wigner function The propagation of the electron wavefunction after interaction with the optical near-field can be described in terms of a unitary evolution operator $\exp \left(-i H_{0} t / \hbar\right)$, where $H_{0}$ is again the free-electron Hamiltonian. Let $\psi_{P}(t)$ be the wavefunction in momentum representation and $p$ the electron momentum in the laboratory frame. The unitary evolution is then given by

$$
\psi_{P}(t)=\mathrm{e}^{-i E_{P} t / \hbar} \psi_{P}(0)
$$

where $E_{p}=c \sqrt{(m c)^{2}+p^{2}}$ is the relativistic energy and $m$ is the electron rest mass. In practice, the electron momenta $p$ after the interaction are all very close to the initial (relativistic) electron momentum $\gamma m v$, where $v$ and $\gamma$ are the initial electron velocity and the Lorentz contraction factor, respectively. For that reason, it is convenient to use 'shifted' momenta defined as $p^{\prime}=p-\gamma m v$.

During the free propagation, the momentum distribution $\left|\psi_{P}(t)\right|^{2}$ remains unchanged because the unitary action only changes phases of the probability amplitudes $\psi_{P}(0)$. In contrast to the momentum distribution, the spatial density distribution will vary in time during the propagation. In a 'shifted' laboratory frame, the spatial representation of the wavefunction is given by the Fourier transformation

$$
\psi(z-v t, t)=\frac{1}{2 \pi} \int_{-\infty}^{\infty} \mathrm{e}^{i p z / \hbar} \psi_{P}(t) \mathrm{d} p
$$

where $\psi_{P}(t)$ is normalized to unity and $z-v t$ is the shifted spatial coordinate. Figure 5.3a in the main text presents a computation of the probability density versus the arrival time of the wavepacket in a given plane as a function of the propagation distance between the 
interaction region and this plane.

The Wigner function of the quantum state (Fig. 5.3b in the main text) is given by

$$
W(z-v t, p, t)=\frac{1}{\pi} \int_{-\infty}^{\infty} \psi_{p+q}(t) \psi_{p-q}^{*}(t) \mathrm{e}^{-2 i q z / \hbar} \mathrm{d} q
$$

It gives a phase space representation of the quantum state [104] and illustrates the propagation-induced pulse compression.

\subsubsection{Numerical calculations}

In order to elucidate the importance of a spatially narrow probing beam and a temporally stretched near-field excitation for the observation of multilevel Rabi oscillations, we performed numerical calculations to quantitatively characterize the influence of an incoherent averaging over (temporally and spatially) varying transition probabilities $P_{N}\left(\boldsymbol{r}_{\perp}, t\right)$, where $t$ is the electron arrival time and $\boldsymbol{r}_{\perp}$ its position vector in the sample plane (perpendicular to the beam direction). In a different context, that is, in the description of laser-electron cross-correlations, similar computations were carried out in refs [98] and [91].

In a simplified geometry, the nanotip is modelled as a straight cylinder of a radius corresponding to that of the tip at the probing position $(r=1 \mu \mathrm{m})$, for which the scattered electric field can be analytically calculated within Mie theory [269]. The field enhancement at the surface of the tip shaft is about 1.4. In the approximated geometry, we obtain a Fourier amplitude of the scattered field of $\frac{g}{F_{\text {inc }}} \approx 80.3 \mathrm{~V}^{-1} \mathrm{~nm}$ (normalized to the incident field $F_{\text {inc }}$ ), in the same order of magnitude as in the experiments, and exponentially depending on the distance to the surface with a radial decay length of approximately $90 \mathrm{~nm}$.

In Extended Data Fig. 5.8a, we study the effect of spatial and temporal averaging on the visibility of the Rabi oscillations by averaging over a disk-shaped beam and a Gaussian temporal distribution of different widths. The upper and lower graphs show the influence of a finite probing area and a reduction of the duration of sample excitation, respectively. Both for larger electron beam widths as well as shorter laser pulses driving the optical nearfield, the Rabi oscillations exhibit weaker modulation and become substantially damped. Therefore, for the experimental electron pulse width of about $800 \mathrm{fs}$ and a field decay of $90 \mathrm{~nm}$, a probe radius around $10 \mathrm{~nm}$ and a near-field duration of $3.4 \mathrm{ps}$ as in the experiments (black lines) allow for the observation of strongly modulated Rabi oscillations. For these 

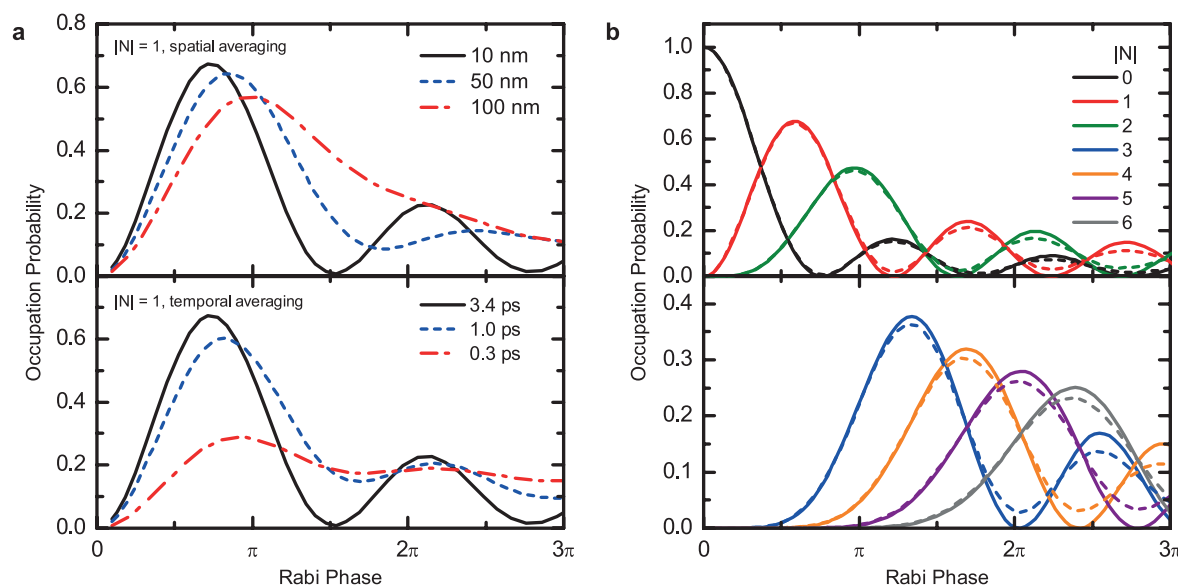

Figure 5.8: Influence of spatial and temporal averaging. a, Effect of electron beam size (top) and laser pulse duration (bottom) on the visibility of the Rabi oscillations in the order $|N|=1$. For increasing electron beam size and decreasing laser pulse duration, the modulations are strongly damped. The black curves correspond to the experimental situation. $\mathbf{b}$, Occupation probabilities of multiple spectral sidebands. Solid lines, $N$ th-order Bessel functions. Dashed lines, numerical calculations accounting for temporal and spatial averaging in the experiments.

experimental parameters, the sideband populations closely follow the analytical Bessel function dependence with minor deviations at higher fields (compare Extended Data Fig. $5.8 b)$.

As shown in the main text, the sinusoidal phase modulation of the electron wavefunction by the interaction with the optical near-field leads to the formation of an attosecond pulse train after a certain distance of free propagation behind the interaction region. In the experiments, the electron pulse consists of a partially coherent ensemble of electrons, and we investigate here the robustness of the attosecond pulse train generation to an incoherent averaging over different coupling constants $g$ and wavefunction evolutions with fluctuating initial energies. We find that an initial kinetic energy spread below the photon energy is fully sufficient for the formation of a clear attosecond pulse structure. Specifically, Extended Data Fig. 5.9 presents evolution maps of the electron pulse structure as a function of propagation distance, incoherently averaging simulations of pure states with an initial kinetic energy width of $0.1 \mathrm{eV}$ each. In Extended Data Fig. 5.9a, b, the electron density is incoherently averaged over a range of kinetic energies $\Delta E=0.7 \mathrm{eV}$ and $2.1 \mathrm{eV}$, respectively. At a range of $0.7 \mathrm{eV}$, the resulting electron density peak is practically indistinguishable from the ideal case of a pure state with $0.1 \mathrm{eV}$ width (solid black line in Extended Data Fig. 5.9e). With increasing spread of the incoherent average, the peak 

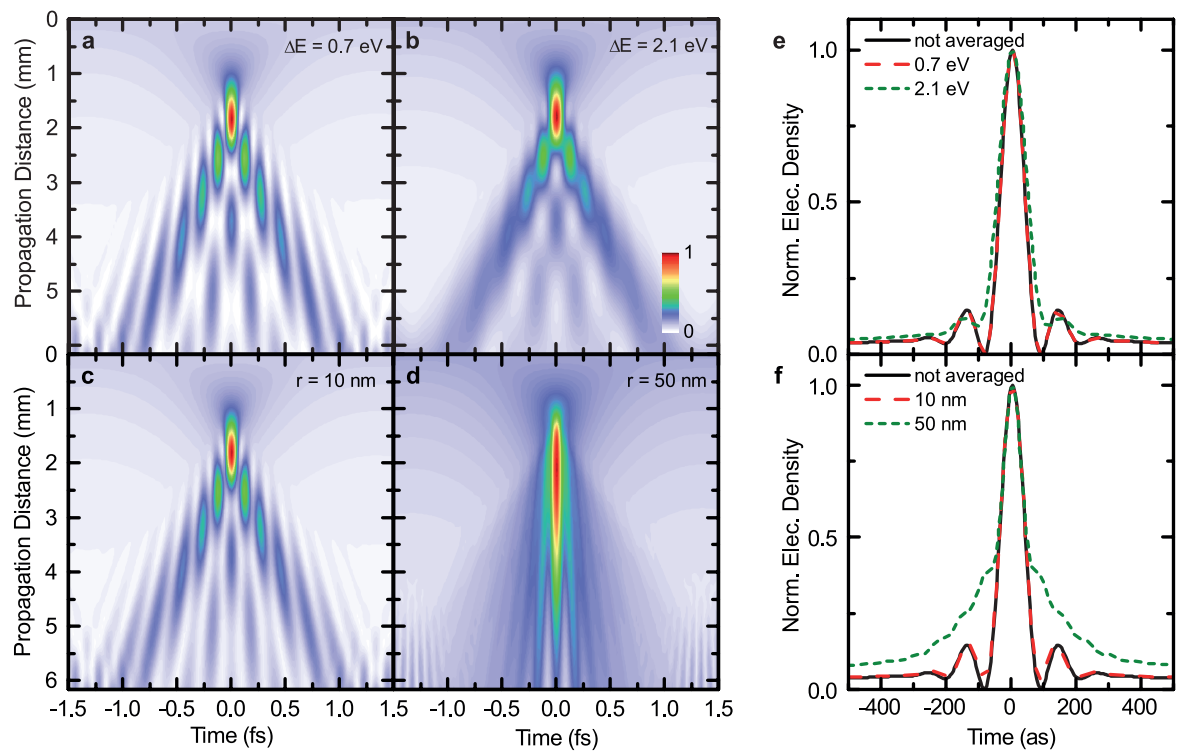

Figure 5.9: Robustness of attosecond pulse train generation. The influence of the electron beam's initial energy spread and lateral size on the temporal peak width of the generated pulse train are shown in the upper and lower rows, respectively. a-d, Evolution of the electron density as a function of propagation distance after the interaction with the optical near- field, incoherently averaged over the initial kinetic energy distribution $(\mathbf{a}, \mathbf{b})$ or the finite probing-area of the electron beam $(\mathbf{c}, \mathbf{d})$. A corresponding line profile at the propagation distance where the electron density peaks are at their maximum and form an attosecond pulse train is shown in $\mathbf{e}$ and $\mathbf{f}$. For the experimental parameters used in this work (energy spread $\Delta E=0.7 \mathrm{eV} \mathrm{FWHM}$ and electron beam radius $r=10 \mathrm{~nm}$ ), the peak width remains nearly unchanged as compared to the ideal (not averaged) case.

begins to smear out, although its duration in the temporal focus is not notably enlarged even for a kinetic energy spread of $2.1 \mathrm{eV}$, three times larger than in the experiment.

An incoherent average over different coupling constants $g$ experienced by the electrons within the electron beam area (lower row) has a different effect: for a small probing radius of $10 \mathrm{~nm}$ and a decay length of the coupling constant as used above, the peak width is not affected, but the depth of the temporal focus is broadened (Extended Data Fig. 5.9c). Increasing the probing radius to $50 \mathrm{~nm}$ (Extended Data Fig. 5.9d), that is, to a size substantially larger than in the experiment, the amplitude of the side lobes grows to ultimately affect the attosecond temporal resolution. In conclusion, the stability to perturbations in the coupling constant and the initial kinetic energy spread demonstrates that attosecond train generation will be observable under the given experimental conditions. 


\section{Acknowledgements}

We thank M. Sivis and B. Schröder for help with sample preparation. We also thank our colleagues within the Göttingen UTEM initiative (C. Jooß, M. Münzenberg, K. Samwer, M. Seibt, C.A. Volkert). This work was supported by the Deutsche Forschungsgemeinschaft (DFG-SFB 1073/project A05), the VolkswagenStiftung, and the Lower Saxony Ministry of Science and Culture. We thank JEOL Ltd and JEOL Germany for their continuing support during the development of the Göttingen Ultrafast Transmission Electron Microscope.

\section{Author Contributions}

The experiments were carried out by A.F., with contributions from J.S. and S.S.; S.S. and C.R. conceived and directed the study; S.V.Y. developed the analytical description and K.E.E. carried out the numerical simulations, each with contributions from A.F., S.S. and C.R.; the manuscript was written by A.F., K.E.E., S.S. and C.R., after discussions with and input from all authors.

\section{Author Information}

Reprints and permissions information is available at www.nature.com/reprints. The authors declare no competing financial interests. Readers are welcome to comment on the online version of the paper. Correspondence and requests for materials should be addressed to S.S. (schaefer@ph4.physik.uni-goettingen.de) or C.R. (cropers@gwdg.de). 



\title{
Chapter 6
}

\section{Nanoscale diffractive probing of strain dynamics in ultrafast transmission electron microscopy}

\author{
A. Feist, N. Rubiano da Silva, W. Liang, C. Ropers and S. Schäfer \\ Struct. Dyn. 5, pp. 14302, January 2018 \\ DOI: $10.1063 / 1.5009822$
}

The control of optically driven high-frequency strain waves in nanostructured systems is an essential ingredient for the further development of nanophononics. However, broadly applicable experimental means to quantitatively map such structural distortion on their intrinsic ultrafast time and nanometer length scales are still lacking. Here, we introduce ultrafast convergent beam electron diffraction with a nanoscale probe beam for the quantitative retrieval of the time-dependent local deformation gradient tensor. We demonstrate its capabilities by investigating the ultrafast acoustic deformations close to the edge of a single-crystalline graphite membrane. Tracking the structural distortion with a $28-\mathrm{nm} / 700$-fs spatio-temporal resolution, we observe an acoustic membrane breathing mode with spatially modulated amplitude, governed by the optical near field structure at the membrane edge. Furthermore, an in-plane polarized acoustic shock wave is launched at the membrane edge, which triggers secondary acoustic shear waves with a pronounced spatio-temporal dependency. The experimental findings are compared to numerical acoustic wave simulations in the continuous medium limit, highlighting the importance of microscopic dissipation mechanisms and ballistic transport channels. 


\subsection{Introduction}

Controlling confined phononic modes in the giga- to terahertz frequency range offers new approaches to steer the flow of heat in nanoscale structures [270] with a broad field of potential applications, ranging from advanced thermoelectric devices [271] to the heat management in dense semiconductor circuits [272]. Furthermore, coupled to tailored light fields, phononic modes with mega- to gigahertz resonance frequencies already developed into essential building blocks in nanometrology [273, 274].

Nanophononics based on tailored multilayer structures has made great progress in recent years, achieving, for example, phonon filtering [275] and phonon amplification [276]. Beyond layered systems, three-dimensionally nanostructured materials facilitate thermally rectifying behavior [277], highly efficient channeled thermal transport across nanoscale vacuum gaps [278-280], enhanced light matter interactions in combined phononic-photonic resonators [281], and phonon lasing [282, 283]. Optical methodologies, such as ultrafast optical spectroscopy [284] and Brillouin scattering [285-287], allowed for experimental access to the spectral and temporal properties of nanophononic systems, including resonance frequencies, dissipation times [79], and nonlinear couplings [288]. However, extracting quantitative information on the structural distortion in nanophononic structures often requires elaborate theoretical modeling. Knowledge of the strain field is essential for tailoring the interaction between phononic fields and other degrees of freedom, such as the coupling of lattice distortions to the electronic [180] and magnetic [289, 290] subsystems, interaction with confined light fields [281], and phase-transitions driven by acoustic [291] and optical [292] phonon fields.

In laterally homogenous samples, ultrafast electron [78, 116, 166, 174, 196, 293-296] and X-ray [26, 177, 297-299] diffraction allows for quantitative access to collective transient lattice distortions. Extending these approaches to three-dimensionally nanostructured geometries remains challenging, despite recent progress in ultrafast coherent diffractive dark-field imaging $[176,300]$ utilizing intense X-ray pulses at free-electron laser facilities [301]. In a table-top approach, ultrafast transmission electron microscopy (UTEM) [31-34, 188-192] provides a visualization of nanophononic modes by time-resolved bright-field imaging [82-85], with first steps towards local diffractive probing [88, 89, 302]. However, the full capabilities of conventional transmission electron microscopy [43, 67-69, 303305] for the quantitative mapping of strain fields have not been harnessed in UTEM.

Here, we demonstrate the quantitative nanoscale probing of optically triggered ultrafast 
strain dynamics in UTEM, employing ultrashort electron pulses in convergent beam electron diffraction (CBED). We achieve a quantitative three-dimensional spatio-temporal reconstruction of the ultrafast lattice distortions in nanoscopic volumes close to the edge of a single crystalline graphite membrane. High-amplitude coherent expansional and shear acoustic waves are launched at the symmetry-breaking sample boundaries, and we track their ballistic propagation and dephasing on nanometer length scales.

\subsection{Ultrafast convergent beam electron diffraction}

In the experiments, we generate low-emittance ultrashort electron pulses by localized photoemission from a tip-shaped field emitter [33, 34, 140]. The femtosecond electron pulses are accelerated to an electron energy of $120 \mathrm{keV}$, coupled into the electron optics of a transmission electron microscope and tightly focused (28-nm focal spot size) onto a 120 -nm thick graphite membrane. For varying probing positions relative to the edge of the membrane, electron diffraction patterns are detected in the far-field [Fig. 6.1(a)]. The sample is optically excited by femtosecond laser pulses focused to a 50- $\mu \mathrm{m}$ focal spot diameter (centered at the graphite edge, $800-\mathrm{nm}$ central wavelength, 50-fs pulse duration, $16-\mathrm{mJ} / \mathrm{cm}^{2}$ fluence). Inhomogeneous structural dynamics are induced on length scales much smaller than the optical focal spot size due to the broken translation symmetry at the nearby sample edge. At an adjustable delay time $\Delta t$ relative to the electron pulse arrival, local structural dynamics are stroboscopically mapped at the electron focal spot position. See Supplementary material for further details on the experimental setup and the graphite sample system.

Figure 6.1(b) displays a typical ultrafast large-angle convergent beam electron diffraction pattern recorded with femtosecond electron pulses before optical excitation $(\Delta t<0)$. In the pattern, the central intense disc-like feature represents the angular distribution of the illuminating electron pulses. Bragg scattering conditions for the graphite lattice planes $(h k l)$ are fulfilled along specific lines in momentum space [38]. At their intersection with the central disc, efficient scattering occurs, forming deficit intensity lines within the disc, and excess lines, which are radially displaced by Bragg angles $\theta_{\mathrm{B}}$ [Figs. 6.1(e) and 6.1(f)].

The angular displacement of each line encodes the length and orientation of a specific reciprocal lattice vector $\mathbf{G}_{h k l}$ and the scattering efficiency encodes the corrugation of the scattering potential $[43,306]$. Thereby, U-CBED gives access to the ultrafast temporal change of local lattice periodicities $d_{h k l}$ and atomic mean-square displacements $\sqrt{\left\langle u^{2}\right\rangle}$ 
(supplementary material, SI 6.8.4). The broad angular range of the incident electron beam (50 mrad full convergence angle) and the chosen sample orientation enable the simultaneous observation of multiple independent Bragg scattering conditions and the corresponding rocking curves [64], providing direct experimental access to the local structural distortion and its temporal evolution.

After optical excitation, we observe pronounced delay-dependent radial Bragg line shifts $\Delta \theta$ (by up to $6 \mathrm{mrad}$ ) in the CBED pattern. For a series of delay-dependent diffraction patterns, see supplementary material movies, M1 and M2. The induced strain dynamics results in no significant azimuthal rotation of Bragg lines for the chosen sample orientation. In the following, we therefore consider the transient changes of Bragg line profiles, obtained by integrating the diffracted intensity along the individual line directions.

In Fig. 6.2, we show the delay-dependent profiles of selected excess Bragg lines for two different probing positions. With the electron focal spot placed at a distance of $r=500 \mathrm{~nm}$ from the edge of the graphite membrane [Fig. 6.2(b)], the (422) and ( $\overline{3} \overline{2} \overline{1})$ lines display a strong multifrequency oscillatory behavior of the average line position and a modulation of the line profile, even including line splittings into multiple components. Other Bragg lines show a different temporal characteristic [e.g., $(\overline{2} \overline{4} 0)$ ] or only very weak overall changes [e.g., (020)]. Remarkably, the recorded transient changes are strongly influenced by the nearby membrane edge. In a continuous part of the film, a much simpler dynamic behavior of the line profiles is observed, as is evident by comparing the transient (422) profiles in Figs. 6.2(a) and 6.2(b).

\subsection{Extracting the deformation gradient tensor}

Disentangling the complex structural dynamics encoded in the ultrafast Bragg line shifts requires a quantitative description of the CBED pattern and its dependence on the distortion of the graphite film. Microscopically, the evolution of the local structural deformation of the membrane is described by the time-dependent tensor field of the deformation gradient $\mathbf{F}(r, \Delta t)=\boldsymbol{\varepsilon}+\boldsymbol{\omega}+\mathbf{I}_{3}$, which can be decomposed into a symmetric strain tensor $\boldsymbol{\varepsilon}$ and an antisymmetric rotation tensor $\omega\left(\mathbf{I}_{3}\right.$ : unit tensor) [43].

Calculating the position of deficit and excess Bragg lines in the CBED pattern requires an adequate description of the Bragg scattering conditions in reciprocal space, which we derive from the graphite unit cell [307] defined by $\mathbf{a}_{1}=a[1,0,0]^{\mathrm{T}}, \mathbf{a}_{2}=a[1 / 2, \sqrt{3} / 2,0]^{\mathrm{T}}$, 
(9)

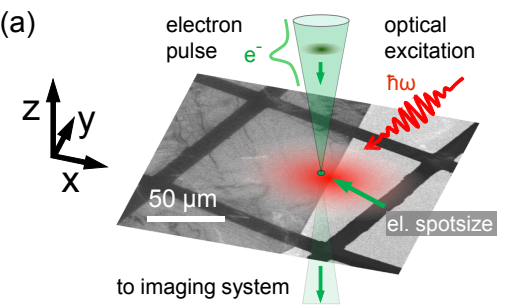

(c)

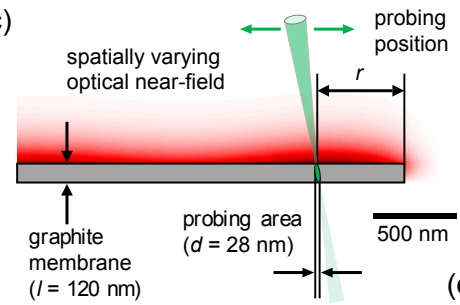

(e) (b)

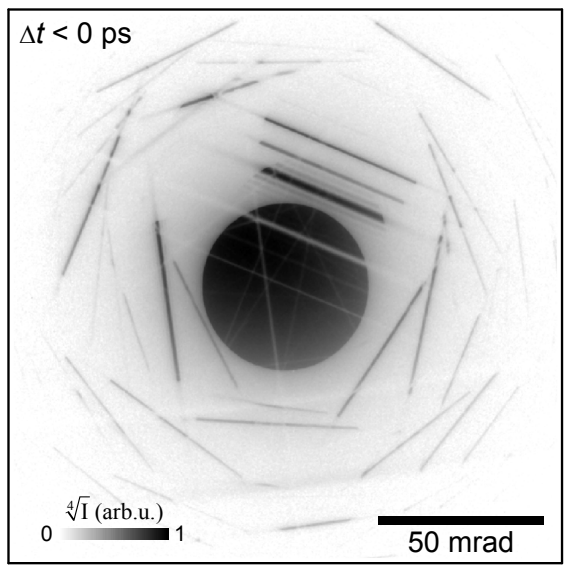

convergent electron

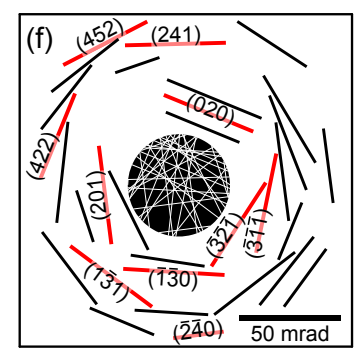

Figure 6.1: Ultrafast convergent beam electron diffraction on single crystalline graphite. (a) Local diffractive probing of optically induced (50- $\mu \mathrm{m}$ laser focal spot size) inhomogeneous structural dynamics in a single crystalline graphite membrane (background image: overview bright field electron micrograph). (b) CBED pattern before optical excitation (exemplary probing position: 500-nm distance to crystal edge). For better visibility of diffraction lines at high scattering angles, the fourth root of the electron intensity $I$ is shown. (c) Experimental geometry of nanoscale probing at the graphite edge. A sharply focused electron beam (28-nm electron focal spot size) maps the local structural dynamics at a variable distance $r$ relative to the edge. Optical interference leads to a slight variation of the excitation profile (sketched in the background). (d) Orientation of in-plane graphite unit cell in real and momentum space with the corresponding coordinate system (orientation of the crystal edge is indicated). (e) Bragg scattering from lattice planes $(h k l)$ results in electron momentum change $\Delta \mathbf{k}=\mathbf{G}_{h k l}$, forming deficit and excess lines in the diffraction pattern. (f) Calculated deficit (white) and excess (black, red) Bragg line positions for the employed sample orientation. For selected Bragg lines, the corresponding Miller indices are given. 
(a)

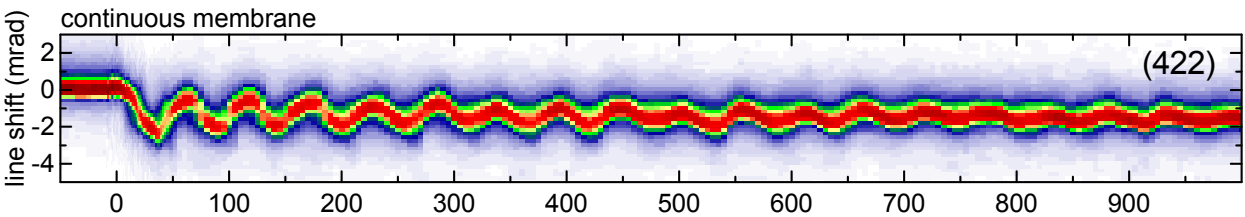

(b)

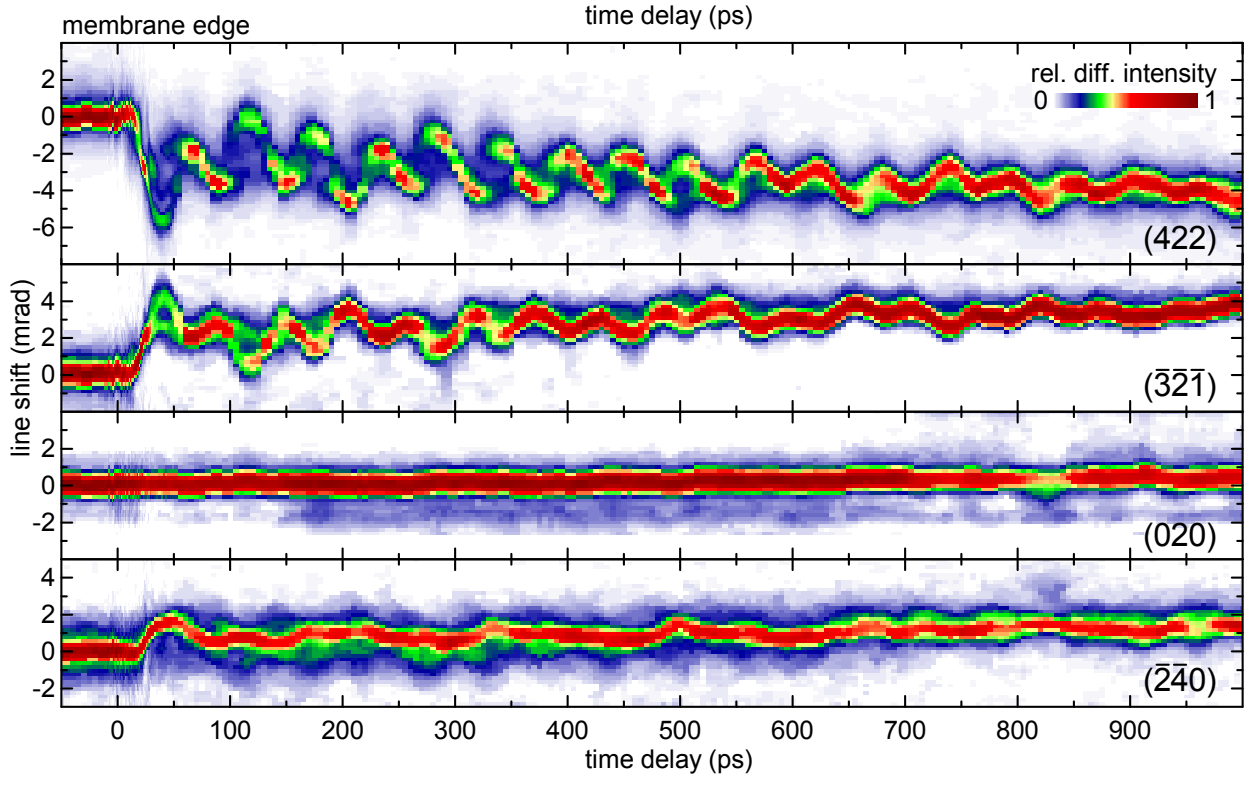

Figure 6.2: Transient modulation of Bragg line profiles. Delay-dependent profiles of selected Bragg lines for probing (a) within a continuous part of the membrane and (b) close to the graphite membrane edge (500-nm relative distance). 
$\mathbf{a}_{3}=c[0,0,1]^{\mathrm{T}}$, with lattice constants $a=2.46 \AA$ and $c=6.71 \AA$. To account for an arbitrary sample orientation, the real space basis, represented by the matrix $\mathbf{B}_{u}=\left[\mathbf{a}_{1} \mathbf{a}_{2} \mathbf{a}_{3}\right]$ in the three-dimensional coordinate system $(x, y$, and $z)$, is rotated [see Fig. 6.1(a)] by applying a matrix $\mathbf{R}=\mathbf{R}_{Z}(\gamma) \mathbf{R}_{Y}(\beta) \mathbf{R}_{X}(\alpha)$, with rotation matrices $\mathbf{R}_{X, Y, Z}$ around a laboratory-fixed coordinate system, $X, Y$, and $Z$ axes, respectively. Taking the planar sample orientation into account, the angles $\alpha$ and $\beta$ correspond to the angular degrees-of-freedom of the double-tilt sample holder, and $\gamma$ is related to the azimuthal orientation of the graphite flake. The reciprocal basis in the laboratory-fixed coordinate system is given by $\mathbf{G}_{\mathrm{u}}=\left(\mathbf{R} \mathbf{B}_{\mathrm{u}}\right)^{-1}$, so that the reciprocal lattice vector with Miller indices $h, k$, and $l$ is expressed as $\mathbf{G}=\mathbf{G}_{\mathrm{u}}[h, k, l]^{\mathrm{T}}$. For scattered and incident wave vectors $\mathbf{k}$ and $\mathbf{k}_{0}$, allowed scattering conditions are obtained from $\mathbf{G}^{2}+2 \mathbf{k}_{0} \cdot \mathbf{G}=0$ by considering the Laue equation $\mathbf{G}=\Delta \mathbf{k}=\mathbf{k}-\mathbf{k}_{0}$ (conservation of momentum) and elastic scattering $\left(\mathbf{G}+\mathbf{k}_{0}\right)^{2}=\mathbf{k}^{2}$ (conservation of energy) [64]. In the paraxial approximation, i.e., $k_{0 X}, k_{0 Y} \ll k_{0}$ and $k_{X}, k_{Y} \ll k$ (for the optical axis chosen along $Z$ ), the scattering conditions can be simplified to

$$
-G^{2} / 2=k_{X} G_{X}+k_{Y} G_{Y}+k_{0} G_{Z}
$$

which describes straight lines $\left[k_{X}, k_{Y}\right]$ in transverse k-space, for each reciprocal lattice vector $\mathbf{G}$. In CBED, the incidence electron spot covers a circular region in the diffraction pattern, and, with the detector plane perpendicular to the optical axis, the allowed scattering conditions are visible as deficit lines with a distance to the origin of $r_{\text {deficit }}=\left(G^{2} / 2-k_{0} G_{Z}\right) / \sqrt{G_{X}^{2}+G_{Y}^{2}}$ and an inclination angle of $\tan (\varphi)=G_{X} / G_{Y}$.

The electrons are scattered into excess lines, which are displaced from the corresponding deficit line by the projected radial scattering vectors $\left[G_{X}, G_{Y}\right]$, so that their radial distance becomes

$$
r_{\text {excess }}=r_{\text {deficit }}+\sqrt{G_{X}^{2}+G_{Y}^{2}}
$$

Bragg line shifts are evaluated by considering changes in their center-of-mass. Additional broadening in the Bragg line profiles due to the inhomogeneous strain distributions is analyzed in Sec. 6.6. For the current sample system, diffusively large-angle scattered electrons only give a minor contribution to the diffraction intensity, so that only a few Kikuchi lines are visible [e.g., the deficit (010) Kikuchi line].

Considering rotation angles $[\alpha, \beta, \gamma]=\left[1.46^{\circ}, 8.05^{\circ}, 22.9^{\circ}\right]$ of the graphite crystal and an initial convergence angle of $25 \mathrm{mrad}$ (half angle), the precise position (radius and 
inclination) of deficit and excess Bragg lines in the diffractograms are reproduced, allowing for an assignment of the indices $h, k$, and $l$ [cf. Fig. 6.1(f)] and further validating the use of the paraxial approximation (all utilized scattering angles are smaller than $80 \mathrm{mrad}$ from the electron optical axis).

A time-dependent distortion of the unit cell can be described by applying the deformation gradient tensor $\mathbf{F}(\Delta t)$ to the undistorted real-space basis of the graphite lattice $\mathbf{B}_{t}(\Delta t)=$ $\mathbf{F}(\Delta t) \cdot \mathbf{B}_{\mathrm{u}}$. Extracting lattice deformations from CBED patterns is a well-established procedure in electron microscopy with continuous beams $[43,308]$, which we now apply to time-resolved diffraction data. Generally, the average unit cell deformation within the electron beam probing volume (cf. Figs. 6.3 and 6.4) can be extracted by applying a forward least squares regression analysis [73, 309], fitting the absolute change in Bragg line positions $\Delta r_{(h k l)}=r_{\text {excess },(h k l), \text { exp }}-r_{\text {excess },(h k l), \text { calc }}(\mathbf{F})$ and inclination angles $\Delta \varphi_{(h k l)}=$ $\varphi_{(h k l) \text {,exp }}-\varphi_{(h k l) \text {,calc }}(\mathbf{F})$ of the most intense lines with the components of the tensor $\mathbf{F}$ as free parameters

$$
\sum_{h, k, l}\left(\Delta r_{(h k l)}(\mathbf{F})\right)^{2}+\sum_{h, k, l}\left(\Delta \varphi_{(h k l)}(\mathbf{F})\right)^{2} \rightarrow \min
$$

No change of the inclination angles $\varphi_{(h k l) \text {,exp }}$ is observed, and we therefore adopt $\Delta \varphi_{(h k l)}=0$ in the fitting procedure.

In our experiment, the radial position of the excess Bragg lines exhibits a high sensitivity to changes of the displacement field $\mathbf{u}$ along the $Z$ direction, mainly related to the components $F_{x z}=(\varepsilon+\omega)_{x z}, F_{y z}=(\varepsilon+\omega)_{y z}$, and $F_{z z}=(\varepsilon+\omega)_{z z}$ of the deformation gradient tensor [310]. Adapting these tensor components, we can quantitatively reproduce the center-of-mass shift of the selected excess lines [cf. reconstructed line positions in Fig. 6.3(c)]. We note that a pure membrane shear along the $x$-axis is described by a finite value of $F_{x z}$ and $F_{z x}=0$, so that $F_{x z} / 2=\varepsilon_{x z}+\omega_{x z}$. The full tensor $\mathbf{F}$ can in principle be determined by additionally analyzing deficit Bragg lines [308, 309] and by recording diffraction patterns along different crystal directions [310].

Generally, in electron diffraction, rocking curves can be strongly affected by multiple scattering processes. However, for the deformation gradient tensor analysis, we only evaluated Bragg lines with extinction lengths $\xi>420 \mathrm{~nm}$, which is significantly larger than the membrane thickness, so that the influence of multiple scattering effects can be neglected and kinematic scattering theory yields a good approximation. 
(a)

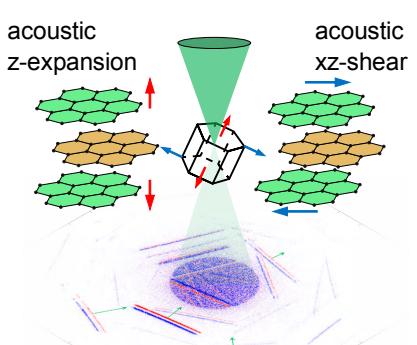

(b)

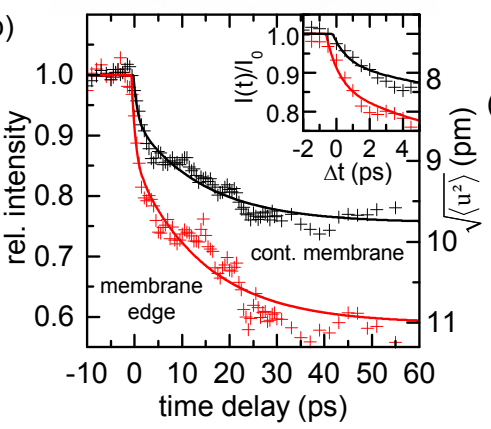

(c)

(d)

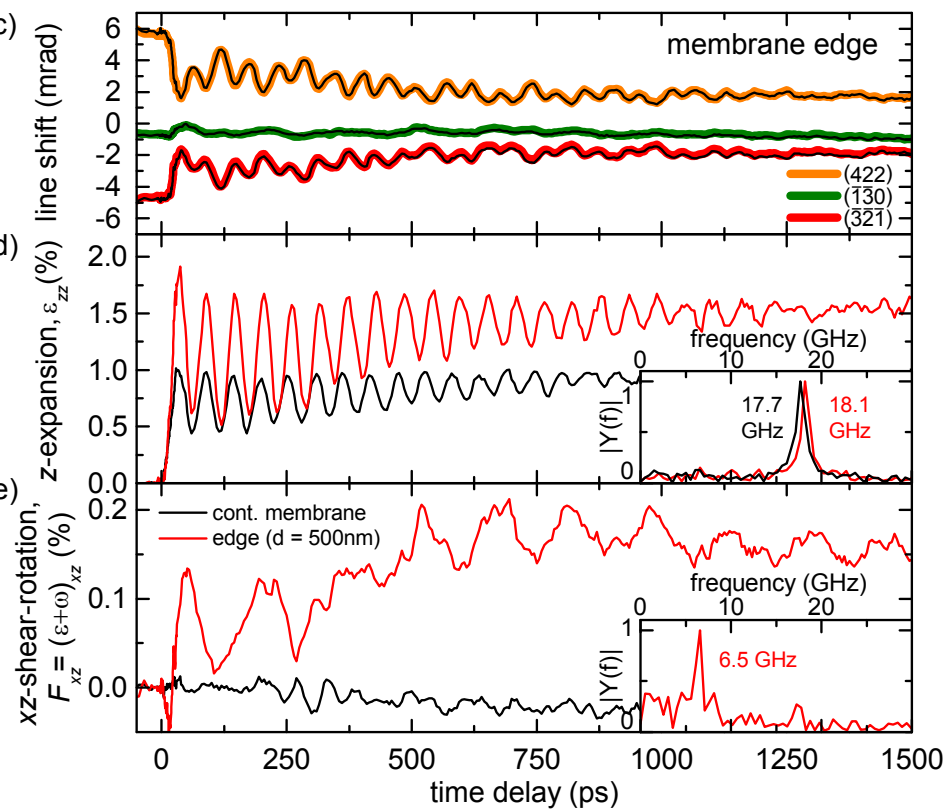

Figure 6.3: Time-dependent Bragg-line changes and dynamics of selected components of the deformation gradient tensor. (a) Local probing of the mean unit cell deformations reveals two dominating mechanical modes: an out-of-plane $z$-axis expansion and an acoustic shear-rotation in the $x z$-plane. (b) Change in (452) Bragg-line intensity and square root of atomic mean square displacement $\sqrt{\left\langle u^{2}\right\rangle}$ in the in-plane direction after optical excitation for probing at the graphite edge (red) and in a continuous part of the membrane (black). (c) Experimentally obtained delay-dependent center-of-mass shift (black line) and reconstructed mean line position (colored line, background) of the (422), ( $(\overline{1} \overline{3} 0)$, and ( $(\overline{3} \overline{2} \overline{1})$ Bragg lines, probed at the graphite edge. (d) and (e) Reconstructed $z$-axis expansion (d) and in-plane $x z$-shear-rotation (e) components (red: membrane edge, black: continuous membrane) with respective Fourier analysis (inset, $|\mathrm{Y}(\mathrm{f})|$ : Fourier amplitude). 


\subsection{Local ultrafast structural dynamics}

A quantitative analysis of the Bragg line shifts in Fig. 6.2 allows us to identify the complex superposition of the acoustic lattice distortions involved in the optically driven dynamics at a homogeneous part of the membrane and at its edge. The local distortion alters the spacing and orientation of crystal lattice planes, resulting in characteristic shifts of Bragg conditions in momentum space [Fig. 6.3(a)]. We extract the components of the local deformation gradient tensor $\mathbf{F}$ for each delay time considering the center-of-mass of multiple experimental Bragg line positions [selected traces shown in Fig. 6.3(c), see supplementary material, SI 6.8.3).

The temporal evolution of the tensor $\mathbf{F}$ near the membrane edge is dominated by two components $F_{z z}(\Delta t)=1+\varepsilon_{z z}$ and $F_{x z}(\Delta t)=(\varepsilon+\omega)_{x z}$ [Figs. 6.3(d) and 6.3(e), red curves], corresponding to an expansional strain along the graphite out-of-plane $z$-axis [for the coordinate system, see Figs. 6.1(a) and 6.1(d)] and a shear-rotation in the $x z$ plane (perpendicular to the membrane edge), respectively. Both deformations leave the $(0 k 0)$ lattice planes unchanged, consistent with the experimentally found negligible transient changes of the (020) line profiles [cf. Fig. 6.2(b)]. Remarkably, the deformation gradient tensor analysis disentangles the multi-frequency temporal behavior of individual Bragg line shifts. The components $\varepsilon_{z z}$ and $(\varepsilon+\omega)_{x z}$ each exhibit damped oscillations at a single frequency, with periods of $T_{\text {expansion }}=56.5 \pm 1.6 \mathrm{ps}$ and $T_{\text {shear-rot }}=154 \pm 5 \mathrm{ps}$ [central frequencies of $17.7 \pm 0.5$ and $6.5 \pm 0.2 \mathrm{GHz}$, see Figs. 6.3(d) and 6.3(e)] for the expansional and shear motion, respectively. Far from the membrane edge $(150-\mu \mathrm{m}$ distance), the optically induced deformation [Figs. 6.3(d) and 6.3(e), black curves] is primarily governed by the expansional out-of-plane motion, and no significant amplitude in the $x z$-component of $\mathbf{F}$ is observed. ${ }^{1}$

The periods of the expansional and shear-rotational distortion, $T_{\text {expansion }}$ and $T_{\text {shear-rot }}$, are given by the roundtrip time of the acoustic waves propagating between the two faces of the membrane. The ratio $T_{\text {shear-rot }} / T_{\text {expansion }}=2.73 \pm 0.16$ is in excellent agreement with the relative magnitude of the corresponding longitudinal and transverse acoustic sound velocities in single crystalline graphite $v(\mathrm{LA}[001]) / v(\mathrm{TA}[001])=(4140 \mathrm{~m} / \mathrm{s}) /(1480 \mathrm{~m} / \mathrm{s})=2.80$ [311]. Furthermore, the periods $T=2 l / v$ yield a membrane thickness of $l=117 \mathrm{~nm}$, which

\footnotetext{
${ }^{1}$ We note that in the continuous film, a low amplitude $\left(\sim 1 \times 10^{-4}\right)$ yz-shear component, i.e., perpendicular to the optical plane of incidence, is observed. Its excitation may be connected to Brillouin scattering processes or additional symmetry-breaking mechanisms, such as anisotropic membrane pre-strain [285].
} 
matches the value of $120 \mathrm{~nm}$ derived by evaluating the thickness fringes [38, 312] of the (010) Bragg line.

At long delay times ( $\Delta t>800 \mathrm{ps})$, the oscillatory membrane expansion becomes strongly damped, approaching an average graphite interlayer distance increase of about $1.5 \%$ at the membrane edge (continuous membrane: $0.9 \%$ ). In order to compare these strain values to a thermal expansion model, we extract the local temperatures from the integrated diffracted intensity change of the (452) Bragg-line after optical excitation [Fig. 6.3(b)]. For an equilibrated phonon distribution $(\Delta t>100 \mathrm{ps})$, a thermal Debye-Waller behavior is reached and we extract an optically induced temperature rise of $\Delta T_{\text {cont }}=270 \mathrm{~K}$ at a continuous part of the membrane and $\Delta T_{\text {edge }}=480 \mathrm{~K}$ at the membrane edge, which corresponds to thermal film expansions of $0.93 \%$ and $1.65 \%$, respectively (see supplementary material, SI 6.8.4). Importantly, ultrafast CBED directly yields full transient rocking curves, so that an acoustic lattice distortion (line shift) and a change in the atomic mean square displacement (line intensity) can be distinguished.

At early delay times, a biexponential drop of diffracted intensity is observed, which is attributed to the previously reported initial non-thermal phonon distribution after optical excitation [75, 313, 314]. This delayed increase in atomic mean square displacement is also reflected in a phase shift of the out-of-plane breathing oscillation. Specifically, we observe the first maximum of $\varepsilon_{z z}$ at $36 \mathrm{ps}$, corresponding to a considerable time lag of about $7 \mathrm{ps}$ relative to a cosine-like transient. The quantitative relation between the non-equilibrium atomic mean square displacement and the resulting stress in the in-plane and out-of-plane directions requires further study, potentially contributing to elucidate the complex hierarchy of energy dissipation in graphite [75, 294, 296, 313-318].

The out-of-plane expansional breathing modes, visible in $\varepsilon_{z z}$, are universal features observed in laser excited thin films as a result of a transient stress gradient $\sigma(z)$ in the depth of the film, with electronic and lattice contributions [76, 284, 294, 296, 319-322]. For the generation of shear modes, as mapped in $F_{x z}$, a symmetry breaking in the lateral direction is required, such as in anisotropic or strained crystal lattices or by local light fields [323-326]. In the following, we will further analyze the mechanism responsible for the coherent generation of these acoustic shear wave components. 


\subsection{Spatio-temporal strain mapping}

In our sample geometry, the structural symmetry is locally broken on mesoscopic length scales due to the presence of the membrane edge. Ultrafast CBED now allows for a local mapping of the evolving deformation gradient tensor field and the sources of the corresponding acoustic waves. To this end, we record time-resolved local diffraction patterns with the focused electron pulses placed at varying distances $r$ from the membrane edge. Figure 6.4(a) exemplarily shows the angular shift of the (201) Bragg line as a function of the delay time $\Delta t$ and the probing position $r$, together with the extracted tensor components $\varepsilon_{z z}(r, \Delta t)$ and $F_{x z}(r, \Delta t)$ [Figs. 6.4(b) and 6.4(e)].

The expansional mode is observed at all probing positions with an equal phase. Its amplitude is spatially modulated and in particular at $r=500 \mathrm{~nm}$ is increased by about $70 \%$ compared to the value found at a larger distance from the graphite edge. This ratio agrees well with the larger temperature rise at this probing position, as observed by the transient Debye-Waller behavior [see Fig. 6.3(b)]. The locally increased sample excitation can be attributed to an interference pattern formed by the optical excitation close to the membrane edge, which is also observable in optically driven inelastic electron scattering, utilizing scanning photon-induced near-field electron microscopy (S-PINEM) [34, 90, 99, 261] [Fig. 6.4(d), supplementary material, SI 6.8.6].

In contrast to the film breathing mode, the shear-rotation component $F_{x z}$ shown in Fig. 6.4(e) exhibits a pronounced spatial dependence. In particular, the onset time of $F_{x z}$ scales linearly with the distance from the membrane edge, with a slope corresponding to a phase velocity of $22 \mathrm{~km} / \mathrm{s}$.

To further analyze the peculiar spatio-temporal strain dynamics, we numerically solve the elastodynamic wave equation for our sample geometry, considering a thermal stress model, a laterally homogeneous sample excitation profile, and graphite bulk properties for the elasticity tensor. The temperature field is obtained by taking into account the inhomogeneously deposited optical excitation and the graphite heat capacity [327]. In addition, diffusional heat transport was included in the model using an anisotropic heat conductivity [327]. For further details on the numerical simulations, see Supplementary material.

For the $\varepsilon_{z z}(r, \Delta t)$ component, we obtain an $r$-independent temporal evolution [Fig. 6.4(c), top], in agreement with the breathing mode of a continuous membrane. In addition, optical excitation results in an in-plane thermal stress $\sigma_{x}$ of the graphite lattice, which 

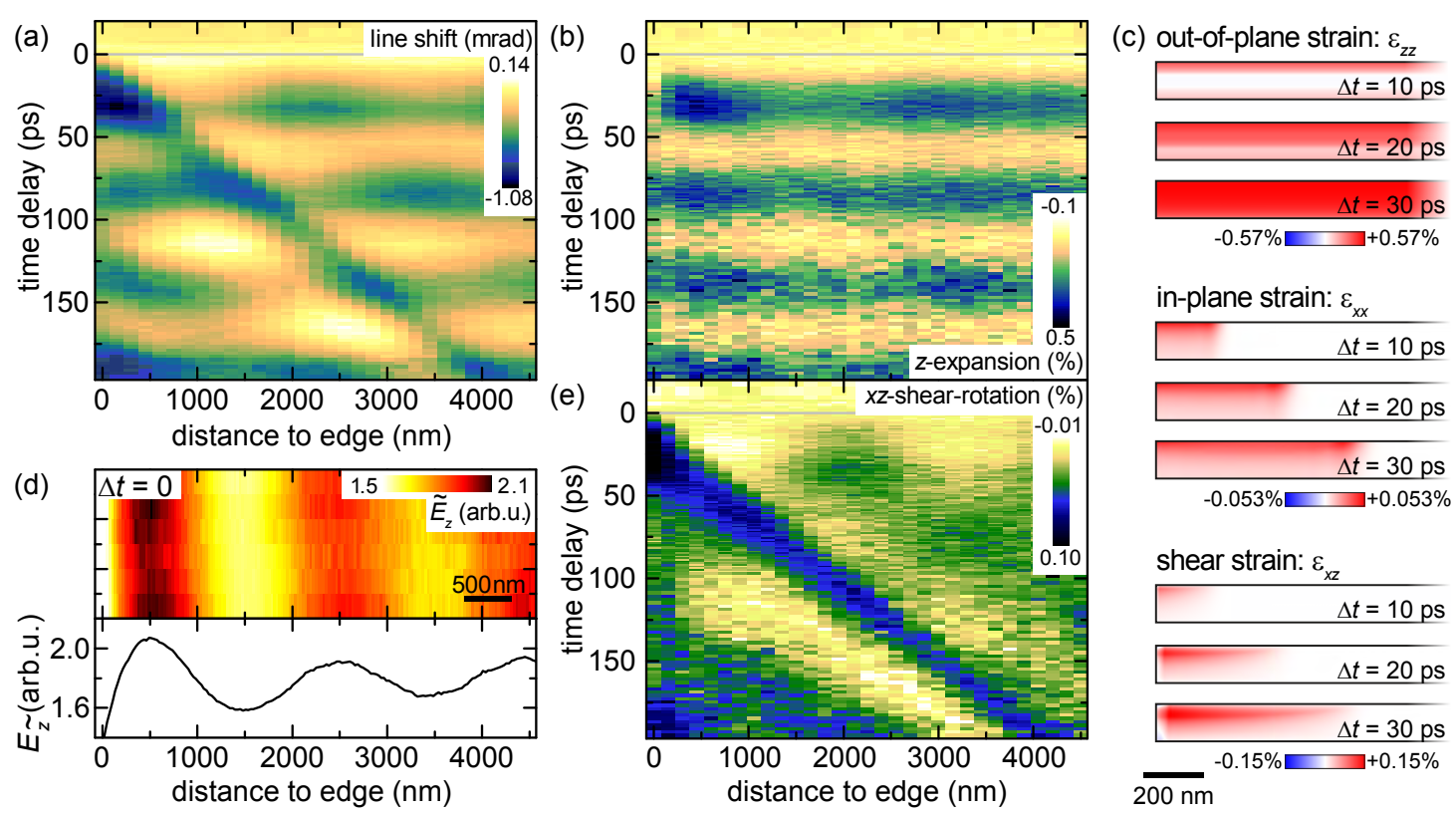

Figure 6.4: Spatio-temporal mapping of near-edge strain dynamics in single crystalline graphite. (a) Mean shift of the (201) Bragg-line as a function of time delay and probing position (recorded at a reduced optical fluence compared to the nonspatially resolved CBED data). Extracted $z$-axis expansion (b) and in-plane shear-rotation (e) retrieved by evaluating the shift of several Bragg lines. (c) Numerical finite-element simulation of the $\varepsilon_{z z}(r, \Delta t), \varepsilon_{x x}(r, \Delta t)$, and $\varepsilon_{x z}(r, \Delta t)$ strain tensor components (absorbed energy density adopted to match experimental $\varepsilon_{z z}$ strain amplitude), illustrating the out-of-plane expansion and the in-plane propagating shock wave within 30 ps after optical excitation. (d) Characterization of the optical near-field structure by scanning photon-induced near-field electron microscopy (S-PINEM), with an optical incidence angle of about $39^{\circ}$.

launches an expansional shock wave in $\varepsilon_{x x}(r, \Delta t)$ from the membrane edge [Fig. 6.4(c), center], propagating perpendicular to the edge with the longitudinal in-plane sound velocity $v(\mathrm{LA}[100])=22.16 \mathrm{~km} / \mathrm{s}$ [311]. Due to the optical excitation profile, the expansional in-plane shock wave is localized to the top of the membrane and thereby induces shearing of the thin film sample. The ultrafast150-lm build-up of shear strain at the top initiates the shear wave travelling back and forth between the membrane faces. ${ }^{2}$ This model readily explains the local excitation of the experimentally observed shear wave with its onset time scaling linear with the distance to the graphite edge.

\footnotetext{
${ }^{2}$ On longer timescales, not considered here, membrane bending modes become excited [83].
} 


\subsection{Transient Bragg line profiles}

Up to here, Bragg line shifts in scanning U-CBED yielded a spatio-temporal map of the lateral structural distortion of the photo-excited graphite membrane. In addition, rich experimental information on the inhomogeneous strain within the depth of the membrane is contained in the profiles of the Bragg lines, which we analyze in the following. Within kinematic scattering theory, a strained crystal imprints a phase modulation onto the diffracted electron wavefront $[69,176]$, resulting in a CBED profile well described by

$$
I\left(\Delta \theta \cdot\left|\mathbf{G}_{h k l}\right|\right) \propto\left|\mathcal{F}\left(e^{\mathrm{i} \mathbf{G}_{h k l} \cdot \mathbf{u}(z)}\right)\right|^{2}
$$

in which $\Delta \theta$ is the change in diffraction angle (relative to the Bragg angle $\theta_{\mathrm{B}}$ ), $\mathbf{G}_{h k l}$ the corresponding reciprocal lattice vector, $\mathbf{u}(z)$ the atomic displacement field, and $\mathcal{F}$ the Fourier transformation along the graphite $z$-axis. The corresponding deformation gradient tensor $\mathbf{F}$ is given (for small deformations, as relevant here) by the gradient of the displacement field, i.e., $\mathbf{F}=\mathbf{I}_{3}+\nabla \mathbf{u}$. Notably, the line profiles depend on the projection $\mathbf{G}_{h k l} \cdot \mathbf{u}(z)$ [Eq. (6.4)], so that the cross sections for individual Bragg conditions are sensitive to different components of the displacement vector field and thereby to the polarization of the involved phonon modes.

In Fig. 6.5, we exemplarily compare the experimental time-dependent (422) line profiles at the membrane edge and in the continuous film with predicted profiles according to Eq. (6.4), utilizing the numerically simulated displacement fields. For the continuous part of the graphite film, a periodic change of the Bragg line width is observed (with a period $T_{\text {expansion }} / 2$ ), which is well reproduced within the numerical strain model [Figs. 6.5(a) and 6.5(c), left panel]. Approximately at delay times of maximum film expansion and compression, sharp Bragg lines are obtained due to the intermediate nearly homogeneous $\varepsilon_{z z}$ strain distribution within the film, as, for example, visible in Fig. 6.4(c) at $\Delta t=30 \mathrm{ps}$. The slight time lag between Bragg line shift and line broadening as well as their relative amplitude sensitively depends on the optical excitation depth and the resulting transient stress profile. In particular, the experimental width of the Bragg line profiles cannot be reproduced if one considers the optical penetration depth in graphite of $\delta_{\mathrm{P}}=36 \mathrm{~nm}$ (Ref. [328]) alone. Instead, a good agreement is obtained for an excitation depth spatially spread to about $90 \mathrm{~nm}$ (see supplementary material, SI 6.8.5), which may be caused by fast interlayer electron or ballistic phonon transport [329, 330]. Furthermore, the asymmetry at 

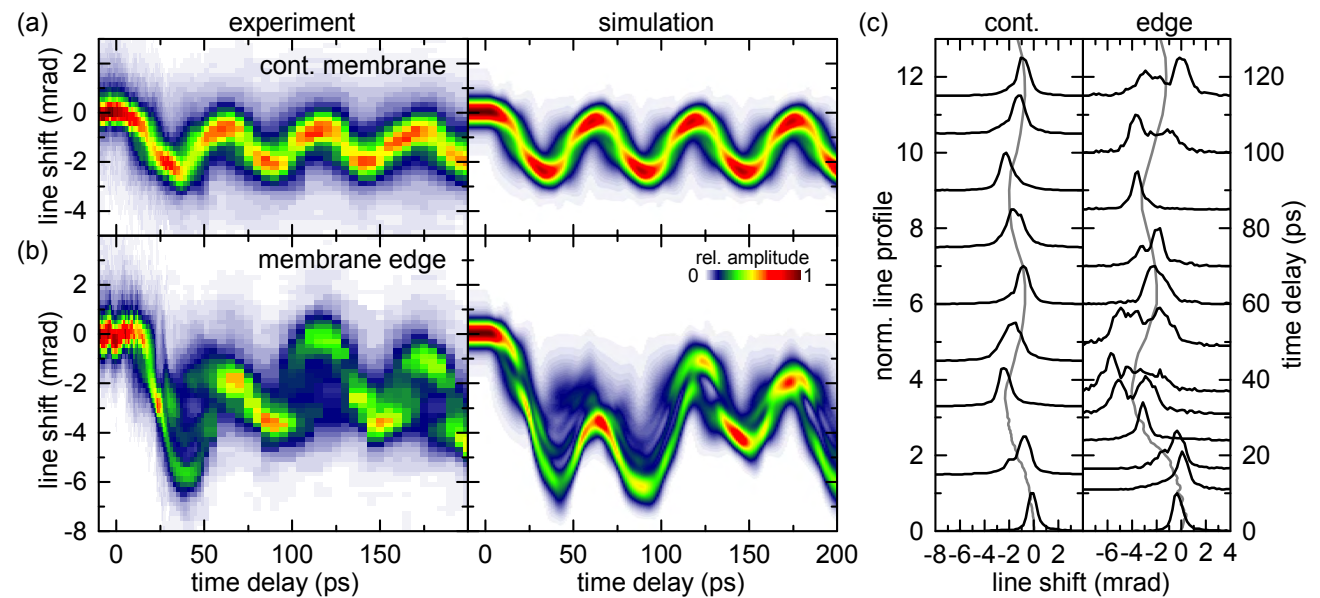

Figure 6.5: Dynamics of the (422) Bragg line profile. Extracted time-dependent cross-sections of the (422) line in (a) a continuous part of the membrane and (b) close to the graphite edge (500-nm distance) compared to calculated line profiles with displacement fields derived from numerical simulations. (c) Normalized line profiles (left-hand axis) at specific time delays (right-hand axis), with mean line position indicated in gray.

the crests of the oscillatory Bragg line movement is reproduced well in the simulations by adopting an 8-ps coupling time of the initial excitation to the experimentally detected coherent out-of-plane motion, similar to the time constant observed for the increase of the in-plane atomic mean square displacement [75, 313, 314].

For the strain dynamics induced at the membrane edge, the more complex behavior of Bragg line profiles [Figs. 6.5(b) and 6.5(c), right panel] is a result of the superposition of expansion and shear deformation, resulting in different projections of the displacement field $\mathbf{u}(z)$ onto reciprocal lattice vectors $\mathbf{G}_{h k l}$ [cf. Eq. (6.4)]. The main features of the experimental line shapes are regained in the numerical strain simulation, including the decreasing intensity maximum after $\Delta t=0$ with a pronounced line sub-structure between 23 and 60 ps [Fig. 6.5(b)]. In addition, also the general experimental trend of partial line re-focusing between 60 and $90 \mathrm{ps}$ and increased broadening between 90 and 140ps is found in the simulation. Microscopically, the line shapes sensitively depend on the relative amplitudes and phases of the expansional and shear wave modes, allowing for a sensitive mapping of nanophononic strain fields. The remaining difference between the experimental and simulated line profiles may indicate the break-down of classical continuum mechanics at the length and time scales considered here. Further developments are required to properly account for the impact of the initial non-thermal phonon distribution and mode specific 
phonon-phonon interactions on ultrafast transport processes and the transient local lattice stress, particularly relevant for the nanoscale geometries considered here.

\subsection{Conclusion}

We demonstrated the quantitative mapping of a time-dependent structural distortion in a nanoscale geometry, utilizing ultrafast convergent beam diffraction with a raster-scanned ultrashort electron probe. Our technique is applicable to a wide variety of locally structured thin-film sample systems. In particular, we believe that U-CBED opens a new avenue for achieving a quantitative description of ultrafast processes relevant in nanophononic devices, potentially allowing for a precise tailoring of nanostructure and function. With the temporal resolution demonstrated here, U-CBED is also capable of imaging phonon modes up to the terahertz regime, which will enable us to address the flow of thermal energy on its intrinsic time and length scales. Such capabilities may help to unravel the influence of local dissipation channels in complex materials, transport processes across designed interfaces, and nonlinear phononic interactions.

\subsection{Supplementary material}

See supplementary material for details on the experimental setup (SI 6.8.1), sample preparation (SI 6.8.2), data collection and analysis (SI 6.8.3), Debye Waller analysis (SI 6.8.4), numerical simulations (SI 6.8.5), and near field characterization (SI 6.8.6) (PDF). Movie showing delay-dependent change in CBED intensity (difference pattern) probed in a continuous part of the membrane (M 1) and close to its edge (M 2) (AVI).

\subsubsection{Ultrafast convergent beam electron diffraction (U-CBED)}

In ultrafast transmission electron microscopy (UTEM), the transient state of an optically excited sample is probed by ultrashort electron pulses. The broad imaging, diffraction and spectroscopy capabilities of state-of-the-art TEM give access to ultrafast dynamics in different degrees-of-freedoms [32, 33, 37].

The Göttingen UTEM instrument is based on a field emission TEM (JEOL JEM2100F), which we modified to allow for optical sample excitation and femtosecond electron pulse generation from a laser-driven photocathode. In our photoelectron gun 


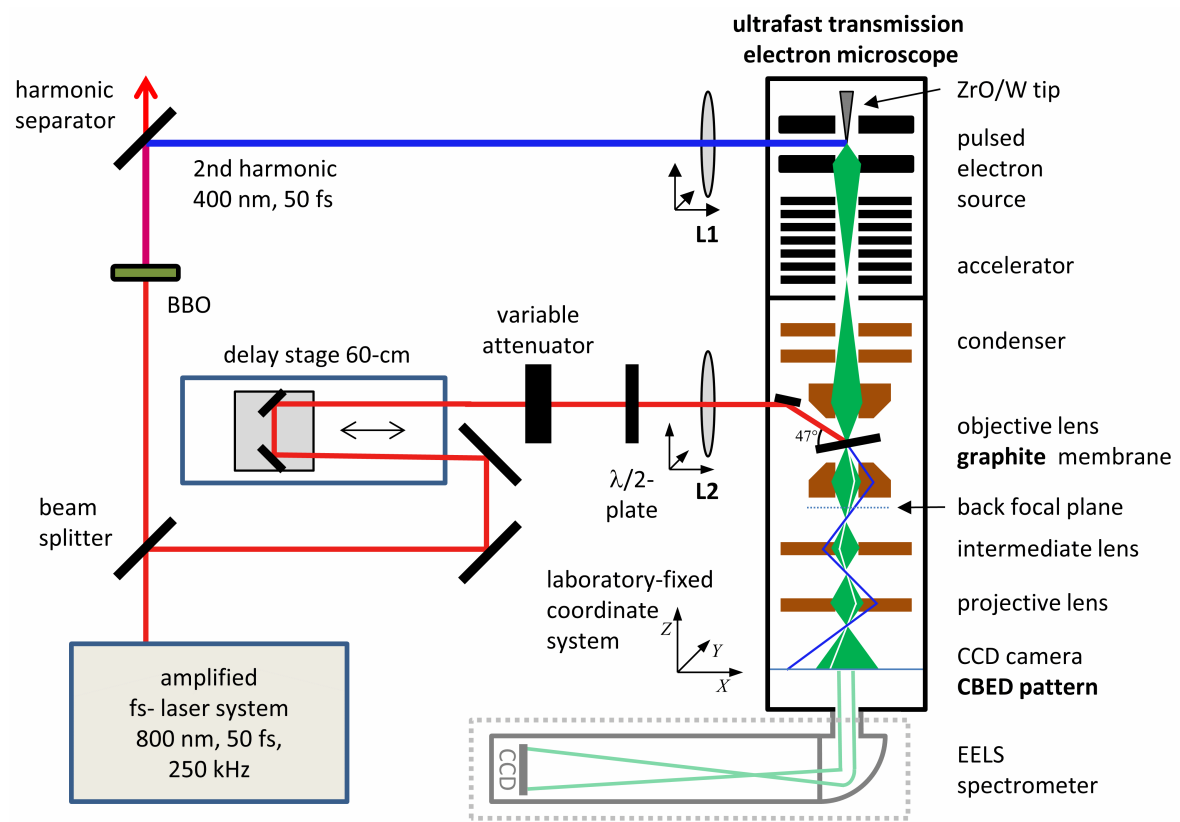

Figure 6.6: Experimental setup. Femtosecond optical pulses from a Ti:sapphire amplified laser system (800-nm central wavelength, 50 -fs pulse duration, $250-\mathrm{kHz}$ repetition rate) are split in two optical paths. The first beam is frequency-doubled and focused (4.1-nJ pulse energy, $\sim 0.3-\mathrm{mJ} / \mathrm{cm}^{2}$ fluence) on the nanoscopic tip emitter for the generation of ultrashort electron pulses. The second beam is attenuated and focused (320-nJ pulse energy, $16.2-\mathrm{mJ} / \mathrm{cm}^{2}$ fluence, $50-\mu \mathrm{m}$ focal spot size, p-polarized, $47^{\circ}$ angle of incidence relative to the sample) with a relative timing set by a mechanical delay stage (see Ref. [33] for more details). The projection system of the TEM is used to record images in real and reciprocal space, as well as electron energy loss spectra with an additional spectrometer.

approach, we make use of localized single-photon photoemission from the apex of a Schottky-type $\mathrm{ZrO} / \mathrm{W}$ field-emission tip at an optical wavelength of $400 \mathrm{~nm}$, yielding low-emittance electron pulses (focus diameter down to $9 \AA$, pulse duration down to 200 fs (full-width-at-half-maximum (FWHM)), energy width of $0.6 \mathrm{eV}$ in the space-charge free regime) [33].

In the current experiment, the photoelectron gun was operated in a high transmission mode [33] at a $250-\mathrm{kHz}$ repetition rate and an acceleration potential of $120 \mathrm{kV}$. At the sample position, the electron beam was focused with a full convergence angle of $50 \mathrm{mrad}$ (200- $\mu \mathrm{m}$ condenser aperture) to a focal spot of $28 \mathrm{~nm}$ in diameter (spot size limited by spherical aberration of the objective lens, spherical aberration coefficient $C_{\mathrm{S}}=1.4 \mathrm{~mm}$, focal length $f=2.7 \mathrm{~mm}$ ), exhibiting a pulse duration of $700 \mathrm{fs}$ (FWHM). The probing position on the sample can be varied with nanometer accuracy using the magnetic deflector 
coils above the objective lens. For optical excitation, p-polarized 50-fs laser pulses were focused onto the sample at an angle of $47^{\circ}$ with respect to the membrane surface. Convergent beam diffraction patterns were recorded on a calibrated charge-coupled device (Gatan Ultrascan 4000$)$, binned to $1024 \times 1024$ pixels $(0.0621 / \mathrm{nm}$ per pixel momentum resolution) with an effective projection camera length of $28.7 \mathrm{~cm}$.

\subsubsection{Sample}

The single crystalline membrane was prepared by mechanically cleaving a graphite mineral crystal (vendor: Naturally Graphite), resulting in 100- $\mu$ m scale sample regions with homogeneous thickness. The graphite flake was supported on a copper grid (square 200 mesh, open areas of $114 \times 114 \mu \mathrm{m}^{2}$ ).

For the probing position close to the graphite edge, we measure a membrane thickness of $120 \mathrm{~nm}$ by evaluating the CBED interference fringes recorded with a continuous electron beam $[38,312]$. Near-edge structural dynamics were acquired at a part of the membrane, in which the cleaving process produced a straight edge oriented along the [120]-direction, i.e. in an armchair-type configuration [331].

For the time-resolved CBED experiments, the specimen was rotated by about $8^{\circ}$ around the graphite $y$-axis and $1^{\circ}$ around its $x$-axis (sample coordinate system, cf. Fig. 6.1d). Hereby, a set of Bragg lines $(h k l)$ is obtained, which yields a high sensitivity to acoustic waves with in-plane polarization parallel and perpendicular to the graphite edge, as well as out-of-plane polarizations. Precise angular alignment was regularly checked by comparing Bragg line positions to numerically calculated diffraction patterns.

\subsubsection{Data collection \& analysis}

All diffractograms were integrated for $5 \mathrm{~s}$, typically containing about $2.5-3.5 \cdot 10^{6}$ electrons. The delay time scans (Figs. 6.1-6.3, 6.5) consisted of 489 steps (75 min measurement time each) and were repeated multiple times (continuous membrane: 10 repetitions, close to the edge: 3 repetitions). For the spatio-temporal map at the graphite edge (Fig. 6.4) the electron focal spot was shifted perpendicular to the graphite edge (41 spatial probing positions) for each individual delay time (159 time steps), resulting in a measurement time of about $16 \mathrm{~h}$.

After normalization to the total number of electrons in each image and flat-field correction for residual aberrations, rectangular regions of interest (ROI) are defined, with one 

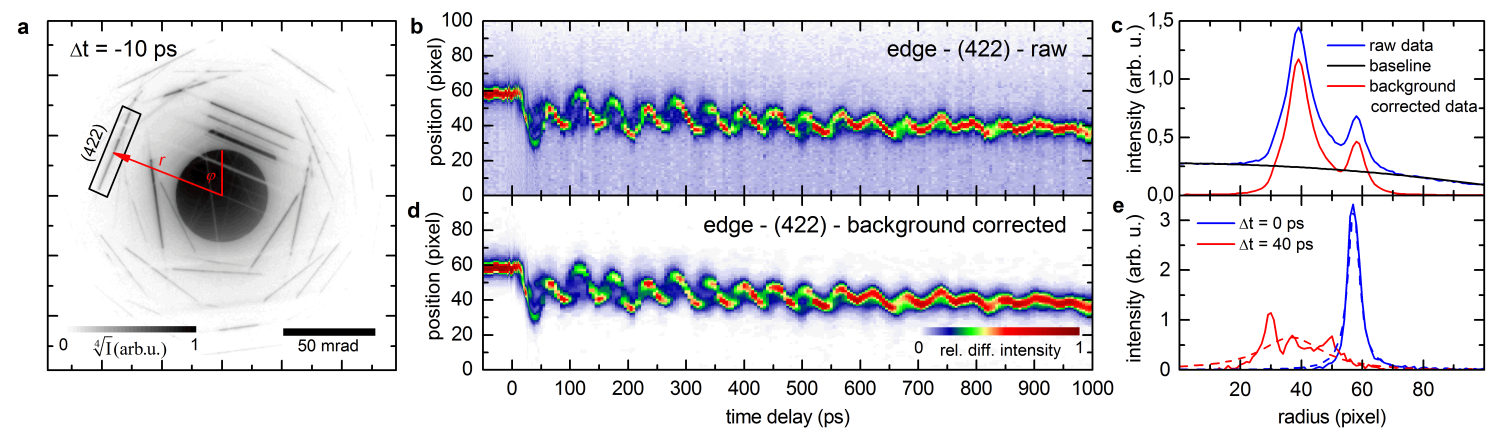

Figure 6.7: Evaluation of the diffraction data. (a) Raw image with indicated region of interest for a specific Bragg line. (b) Extracted line profile from raw data as function of time delay. (c) Removal of global background from delay-time integrated intensity of (b) (after normalization to the total number of electron per image). (d) Time dependent line-profiles after background removal and median filtering (along $r$-axis). (e) Exemplary line profiles (solid) with Lorentzian fit (dashed). Non-broadened profiles (e.g. $\Delta t=0 \mathrm{ps}$, blue) can be approximated by a Lorentzian function, while center-of-mass and standard deviation analysis are crucial for inhomogeneously broadened lines (e.g. $\Delta t=40 \mathrm{ps}$, red)

edge parallel to the respective Bragg line ( $h k l$ ) (cf. Fig. 6.7a). For each time step, a line profile is extracted (Fig. 6.7b) by rotating the image parallel to the respective line and integrating the intensity within the ROI along the line direction. For each ROI, a delay-time averaged quadratic background is subtracted (Fig. 6.7c,d). Amplitude, position and width of the Bragg lines are either extracted by a Lorentzian fit (Fig. 6.7e, applied for weakly broadened line profiles, e.g. (020), and for all lines at low optical excitation density), or by calculating the center of mass and standard deviation (non-Lorentzian line shapes, e.g. (422) for a high excitation density).

\subsubsection{Debye Waller analysis}

In order to extract the optically induced temperature rise of the graphite membrane, we analyzed the decrease of the integrated diffraction intensity $I$ within the (452) Bragg line (Fig. 6.3b). At late delay times, a quasi-equilibrium phonon temperature is established, resulting in a nearly constant intensity drop of $41 \%$ at the edge and $25 \%$ in the continuous film, respectively (Fig. 6.8a,b). Membrane cooling occurs on nanosecond to microsecond time scales, and is not observed in the temporal window considered here.

For thermalized phonon distributions, the relative intensity decrease $I\left(T_{0}+\Delta T\right) / I\left(T_{0}\right)$ at temperatures $T_{0}$ and $T_{0}+\Delta T$ is given by $\exp \left(2\left(W\left(T_{0}\right)-W(T)\right)\right)$, in which $W(T)$ is the 
Debye-Waller factor. In the Debye model $W(T)$ can be expressed as [332]

$$
W(T)=\frac{\left\langle u_{1}^{2}\right\rangle G^{2}}{4}=\frac{3 \hbar^{2} G^{2}}{2 m \mathrm{k}_{\mathrm{B}} \Theta_{\mathrm{D}}}\left[\frac{1}{4}+\left(\frac{T}{\theta_{\mathrm{D}}}\right)^{2} \int_{0}^{\theta_{\mathrm{D}} / T} \frac{s}{\exp (s)-1} \mathrm{~d} s\right]
$$

where $\left\langle u_{1}^{2}\right\rangle$ is the atomic means square displacement, $m$ is the atomic mass of carbon, $\mathrm{k}_{\mathrm{B}}$ the Boltzmann constant, $\hbar$ the reduced Planck constant and $\theta_{\mathrm{D}}$ the Debye temperature. For the chosen Bragg condition, the intensity decrease is dominated by thermal in-plane vibrations $\left(\Theta_{\mathrm{D}} \approx \Theta_{\mathrm{D}}\right.$, in-plane $\left.=1300 \mathrm{~K}\right)$ [332], resulting in an approximately linear temperature dependence for the relevant temperature range (Fig. 6.8c). The total drop of diffracted intensity at later delay times $(\Delta t>100 \mathrm{ps})$ corresponds to a laser induced increase of the lattice temperature from $T_{0}=300 \mathrm{~K}$ to about $\Delta T_{\text {edge }}=480 \mathrm{~K}$ at the edge and $\Delta T_{\text {cont }}=270 \mathrm{~K}$ in the continuous film, respectively.

As a cross check, we estimate the optically induced temperature increase from the known material constants and the incident pump fluence. Specifically, for an optical reflectivity of $25 \%$ (p-polarized, $47^{\circ}$ incidence angle), we obtain an absorbed fluence of $8.4 \mathrm{~mJ} / \mathrm{cm}^{2}$, resulting in a temperature increase in the continuous part of the membrane $\Delta T_{\text {cont }}$ of $225 \mathrm{~K}$ $-450 \mathrm{~K}$, depending on the base temperature of the membrane $(300 \mathrm{~K}-800 \mathrm{~K})$. We note that at the employed laser repetition rate, an increase of the membrane base temperature is expected, depending on the thermal contact between the graphite flake and the supporting copper grid.

In our experimental data, we can directly quantify the increase in atomic mean square displacement in the in-plane direction, by extracting the temporal change of overall diffraction intensity into a specific Bragg line, as shown in Fig. 6.3b. For early delay times, a biexponential decay behavior with time constants of $\tau_{1}=850(800) \mathrm{fs}$ and $\tau_{2}=14.6(14.9) \mathrm{ps}$ is observed at the membrane edge (continuous membrane). The extracted time scale of the fast component represents an upper bound, due to the convolution of the intrinsic dynamics with the 700-fs electron pulse duration (FWHM). This biexponential behavior was previously attributed to the thermalization of a non-equilibrium phonon distribution [75, 313, 314] generated by the ultrafast decay of strongly coupled optical phonons (SCOPs) [75, 294, 313-316].

Considering the out-of-plane thermal expansion coefficient of graphite $\alpha=29 \cdot 10^{-6} 1 / \mathrm{K}$ [333], the observed change in interlayer spacing of $0.9 \%$ in the 

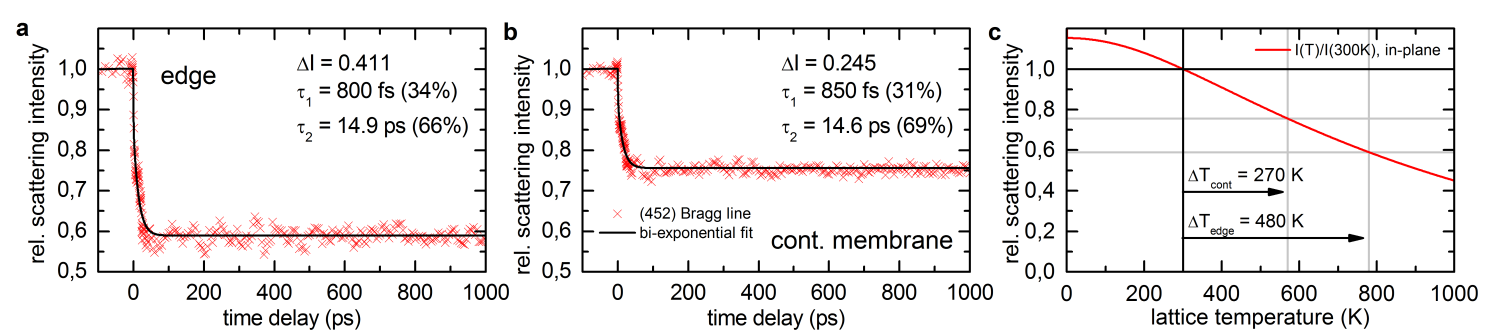

Figure 6.8: Intensity change of the (452) Bragg line. After ultrashort excitation with an illuminating fluence of $16.2 \mathrm{~mJ} / \mathrm{cm}^{2}$, the scattering efficiency of the (452) Bragg line decreases in a biexponential behavior both at the edge (a) and in the continuous film (b). Time constants $\tau_{1}$ and $\tau_{2}$ obtained from bi-exponential fit. Ratio of fast/slow component relative to the overall intensity drop $\Delta I$ given in parenthesis. The different magnitudes of the overall drop (a) at the edge $(41.1 \%)$ and (b) in the continuous film $(24.5 \%)$ reflects the difference in local temperature. (c) The scattering intensity for the (452) Bragg line drops with an increase of equilibrium lattice temperature after laser heating of the graphite membrane (considering the in-plane Debye temperature).

continuous part of the membrane and $1.5 \%$ close to its edge correspond to a temperature increase of $\sim 310 \mathrm{~K}$ and $\sim 520 \mathrm{~K}$ respectively, in reasonable agreement with the temperatures extracted from the Debye-Waller analysis.

\subsubsection{Numerical simulation of graphite thin film lattice dynamics}

To complement our experiments, we calculate quantitative Bragg line profiles from the inhomogeneous strain distributions derived from numerical simulations (Figs. 6.4c and $6.5 \mathrm{a}, \mathrm{b}$, right panel). To this end, we solve the elastodynamic wave equation in a $2 \mathrm{D}$ finite element simulation (COMSOL Multiphysics 5.3, simulated slab size of $117 \mathrm{~nm} \times 8 \mu \mathrm{m}$ ) in response to an optically induced thermal stress $\sigma_{i j}(x, z, t)=\Delta T_{\mathrm{eff}}(x, z, t) \cdot \sum_{k} C_{i j k k} \alpha_{k k}$ [324], considering graphite bulk properties for the elasticity tensor $\mathbf{C}$ [311], and the diagonal components of the thermal expansion tensor $\alpha_{x x, y y, z z}=[1,1,29] \cdot 10^{-6} \mathrm{~K}[333]$.

For the time-dependent temperature distribution, we include the initially inhomogeneously absorbed optical energy density and subsequent heat diffusion [327]. From the resulting time- and position-dependent, three-dimensional displacement field $\mathbf{u}(x, z, t)$, we calculate the diffraction rocking curve of a specific Bragg scattering condition $(h k l)$, according to Eq. 6.4 in the main text (cf. Ref. [69, 176]).

In a first model for the temperature field $T_{\text {eff }}(x, z, t)$, we utilized the depth-dependent absorbed optical power $Q(z, t)$ in the membrane, including the optical absorption length of 
graphite $\left(\delta_{\mathrm{P}}=36 \mathrm{~nm}\right)[328]$ at the employed wavelength and incidence angle, and FabryPerot interference at the membrane faces. To phenomenologically model the coupling time between initial optical excitation and out-of-plane expansional motion, we assumed $Q \propto \exp (-t / \tau)$, with $\tau=8 \mathrm{ps}$. While this model generally reproduces the overall Bragg line displacements (observed in the continuous membrane) as well as the asymmetry at the crests of the Bragg line oscillations, the line splitting is largely overestimated. A good match with the experimental profiles is obtained, when spreading the optically excitation $Q$ to about $90 \mathrm{~nm}$ within the depth of the film (Fig. 6.5a). A possible mechanism is the fast ballistic transfer of heat, in qualitative agreement with theoretical predictions [329] and experimental findings [330] for the thermal phonon mean-free-path at room temperature along the out-of-plane direction in the $100-\mathrm{nm}$ range. We note, that such a process also contains a temporal component governed by the group velocity of thermal phonons in the out-of-plane direction, which warrants further studies.

In addition, using a thermal stress model, a good agreement with the experimental line splittings at the membrane edge is also found (Fig. 6.5b). To account for the lateral inhomogeneous optical excitation field, we scaled the strain tensor components to match the experimental amplitude of the expansional and shear mode at the edge. A better fit to the experimental profiles is found, if adopting a shorter optical excitation depth of $36 \mathrm{~nm}$ and a coupling time $\tau=1 \mathrm{ps}$ for the in-plane component of the displacement field, suggesting a strong mode-specificity of the Grüneisen parameter [322] for the early non-equilibrium phonon distribution.

\subsubsection{Characterization of the optical near-field structure at the graphite edge}

In order to connect the experimentally observed, laterally inhomogeneous strain $\varepsilon_{z z}$ to the optical near-field at edge, we characterize the local optical field distribution at time zero (Fig. 6.4d) by a scanning variant [34, 261] of photon induced near-field electron microscopy (PINEM) [34, 90, 91, 98, 99]. This technique gives access to a specific spatial Fourier component of the optical field component $E_{z}$ along the propagation direction of the electron beam, which is quantified by a coupling constant $|g|$, serving as a measure for the nanoscale structure of the scattered light field at the sample. Specifically, with focused electron probe pulses, we record electron energy spectra at a varying distance from the membrane edge (cf. Fig. 6.9a). Under optical illumination (800-nm central wavelength, dispersively stretched 3.4-ps optical pulse duration) and at $\Delta t=0$, the spectra exhibit 

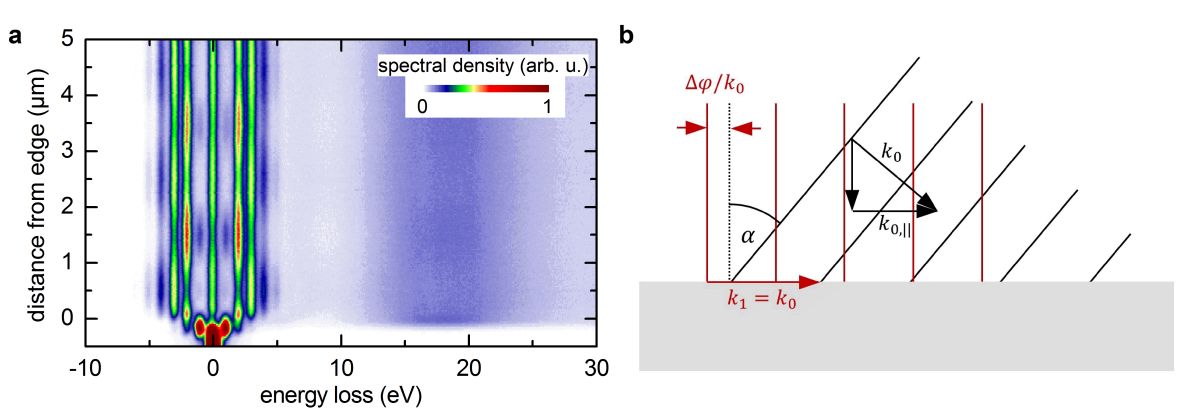

Figure 6.9: Characterization of the optical near-field at the graphite edge. (a) Normalized electron energy loss spectra (EELS) at spatial and temporal overlap of electron and laser beam as a function of distance from the edge. The near-field extends into the vacuum, which is evident by comparing the spatial extend of photon-scattering and the scattering from graphite bulk plasmons at higher energy losses $(>10 \mathrm{eV})$. (b) The spatial variation of the optical amplitude is caused by the interference of the impinging light field with the scattered wave from the graphite edge. The maxima reoccur for $d=1 /(1-\sin (\alpha))$.

multiple sidebands spaced by the photon energy with the population of sidebands related to $|g|[34,91,98]$.

As shown in Fig. 6.4d, we find the coupling constant to vary with the distance to the graphite edge in the form of a damped oscillation with a $2-\mu \mathrm{m}$ spatial periodicity. Generally, optical scattering from the edge of thin film is a complex problem, but the spatial periodicity of the light field at the surface can be obtained by considering the interference of the incident illumination wave vector $\mathbf{k}_{0}=2 \pi / \lambda$ with the light field scattered at the edge with wave vector $\mathbf{k}_{1}$. The interfering light field exhibits a beating in its amplitude along the interface with a periodicity $d$ given by the difference wave vector $|\Delta \mathbf{k}|=2 \pi / d=\left|\mathbf{k}_{0, \mid}-\mathbf{k}_{1}\right|$, where $\mathbf{k}_{0, \mid}$ is the projected incident wave vector onto the interface. With $\left|\mathbf{k}_{1}\right|=\left|\mathbf{k}_{0}\right|$ and $\left|\mathbf{k}_{0,||}\right|=\left|\mathbf{k}_{0}\right| \cdot \sin (\alpha)$, the periodicity $d=1 /(1-\sin (\alpha))$ is obtained. For the experimental incidence angle $\alpha$ of about $39^{\circ}$, a spatial periodicity $d$ of $2.16 \mu \mathrm{m}$ is expected in reasonable agreement with the scanning PINEM maps. For the ultrafast scanning CBED experiments (Fig. 6.4a,b,e), an optical incidence angle of $47^{\circ}$ was used, resulting in a modulated optical field strength with a periodicity of about $3 \mu \mathrm{m}$, closely reproducing the observed spatially modulation in the expansional mode amplitude (Fig. 6.3b). 


\section{Acknowledgments}

We gratefully acknowledge funding by the Deutsche Forschungsgemeinschaft (No. DFGSPP- 1840 "Quantum Dynamics in Tailored Intense Fields" and No. DFG-SFB-1073 "Atomic Scale Control of Energy Conversion," project A05), support by the Lower Saxony Ministry of Science and Culture, and funding of the instrumentation by the DFG and VolkswagenStiftung. W.L. would like to acknowledge support by the Director Fund of WNLO (Grant No.WNLOZZYJ1501) and the National Natural Science Foundation of China (Grant No. 11574094). 


\section{Chapter 7}

\section{Discussion}

The following sections summarize the key results presented in Chs. 4-6 respectively and discuss their implications on the quickly evolving field of ultrafast electron microscopy. This thesis centers on the implementation of a novel electron emitter concept for UTEM, the quantum coherent manipulation of free-electrons by light and the nanoscale diffractive probing of ultrafast local strain dynamics.

\subsection{Ultrafast TEM using coherent electron pulses}

Chapter 4 described the first implementation and projected future applications of an advanced UTEM instrument relying on high-coherence ultrashort electron pulses. Specifically, a laser-driven Schottky field emitter is integrated into a conventional TEM, allowing for local linear photoemission from the nanoscale front facet of a zirconium oxide covered, single-crystalline tungsten tip. For a range of operation conditions, the electron beam properties are systematically characterized in terms of the occupied transverse and longitudinal phase space distributions. In the space-charge free regime, unprecedented electron pulse properties are obtained, combining a $9 \AA$ electron focal spot diameter with $200 \mathrm{fs}$ pulse duration and $0.6 \mathrm{eV}$ spectral width. A peak brightness of the electron beam of up to $1.7 \cdot 10^{13} \mathrm{~A} / \mathrm{m}^{2} \mathrm{sr}$ and a transverse degree of coherence of $11 \%$ are demonstrated. The advanced ultrafast imaging, diffraction and spectroscopic capabilities of the Göttingen UTEM instrument are illustrated by selected applications using a photoelectron beam. Finally, opportunities for the coherent control of free-electrons by optical near-fields are briefly addressed. 


\subsubsection{Comparison of ultrafast electron gun concepts}

This section attempts a critical review of current ultrafast electron gun technology. In previous implementations of short pulsed electron sources, the emitter geometry and operation conditions were optimized to fit the electron beam requirements of specific experiments (usually a subset of the design considerations mentioned in Sec. 3.3). Different studies put emphasis on the transverse or longitudinal beam emittance or brightness, the electron pulse charge, spectral width or duration, or highlight an advantageous trade-off of these. Conceptually, the intrinsic electron source properties can be treated separately from the subsequent phase space evolution. Central challenges are reversible and nonreversible phase space degradation due to propagation and space charge effects. Mitigation is provided by applying high acceleration fields and potentials, and tailored phase space manipulation by spatial filtering or compression schemes. The two main design pathways are discussed in the following and Figure 7.1 compares the different ultrafast electron gun concepts.

\section{High-charge pulses for single (few) shot probing}

For all non-reversible dynamics and in cases where the total electron beam current at limited repetition rates needs to be maximized, the generation of high-charge electron bunches is required. Typically, the photoemission current is increased to a value still yielding acceptable electron beam properties for the specific experiment.

A compact electron gun scheme for diffraction studies uses photoemission from flat metal cathodes. High static acceleration fields (up to $12.5 \mathrm{MV} / \mathrm{m}$ ) and potentials (up to $100 \mathrm{kV}$ ) [201, 214] enable down to $100 \mathrm{fs}$ pulse durations at up to $5 \cdot 10^{3} \mathrm{e}^{-} /$pulse for short emitter-sample distances. For flat photocathodes, the transverse beam emittance can be reduced by a initial narrow kinetic energy distribution [133, 198-201] and a minimized laser focal spot diameter [201, 202, 334] (cf. section 4.3.1). Furthermore, photoemission from ultracold plasmas with electron temperatures down to $10 \mathrm{~K}$ [203] yields 25-ps electron pulses of up to $10^{3}$ electron and an normalized emittance of $1.5 \mathrm{nmrad}$ [335].

Increasing the pulse charge while maintaining reasonable beam properties necessitates higher acceleration fields or schemes to re-compress the longitudinal phase space distribution. Both can be achieved by employing radio-frequency (RF) cavities (cf. Sec. 3.2). Conventional photoelectron guns (operating at $\sim 100 \mathrm{keV}$ ) are equipped with RF-cells to compress the longitudinal phase space, as shown by numerical simulations $[111,119,120$, 

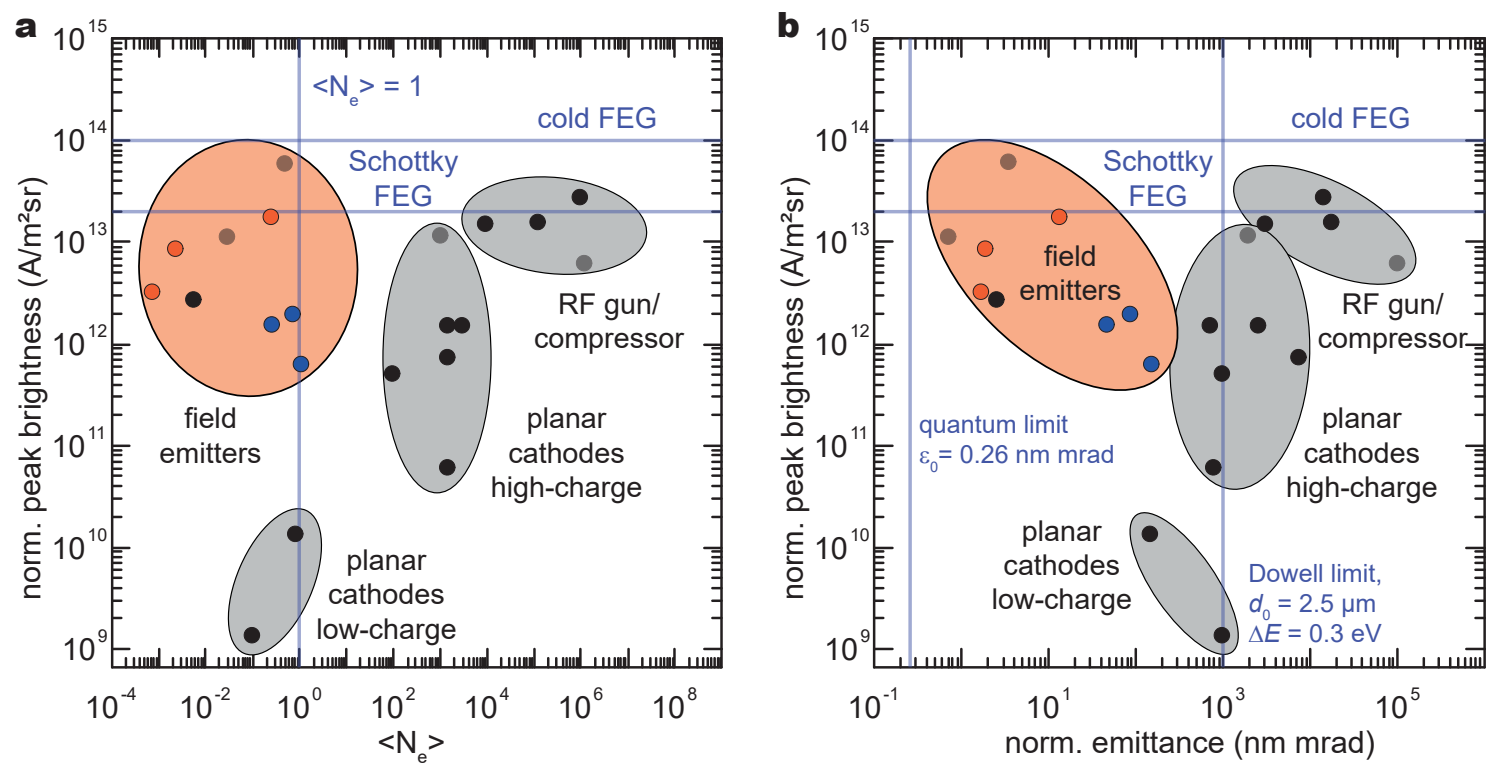

Figure 7.1: Comparison of ultrafast electron gun concepts. As figure-of-merit, the normalized peak brightness determines the usability of an electron beam for most practical applications, here given as (a) function of the average number of electron per pulse $\left\langle N_{e}\right\rangle$ and (b) the transverse normalized beam emittance $\varepsilon_{\mathrm{n}, \mathrm{rms}, r}$. In the high-coherence single-electron limit, nanoscale tip-emitters feature an unparalleled low emittance and high brightness (this thesis: colored dots; "high coherence" (orange) and "high transmission" (blue) mode, cf. section 4.3.1). Singleshot and low-repetition rate applications require high charge electron pulses from large-area photocathodes, with strong benefit from RF-compression and RF-gun technology. See text for references (gray dots: assumed value, when no full beam characterization is given).

$336]$ and in experiments [111, 119, 228, 232, 337, 338], e.g. yielding electron bunches of $10^{5}-10^{6}$ electrons with 100 fs duration (FWHM) [338].

Alternatively, a gun design by Daoud et al. accelerates electrons to an energy of $137 \mathrm{kV}$ while compressing the pulses in an RF-cavity, delivering 60-fs pulses that contain $10^{6}$ electrons and feature a transverse degree of coherence of $K=7.2 \cdot 10^{-6}$ [118]. One of the major challenges for RF-cavity acceleration and compression is the timing jitter of laser pulses and the RF-phase, which was just recently improved to $<50 \mathrm{fs}$ for external synchronized triggering [338].

Extending this concept, several projects make use of RF-photoinjector gun technology (originally developed for the seeding of particle accelerators and free-electron lasers) to create ultrashort relativistic electron pulses of $1-5 \mathrm{MeV}$ kinetic energy and acceleration fields of up to $100 \mathrm{MV} / \mathrm{m}[115,116,233,339-341]$. Due to the strong acceleration, pulses of up to $10^{5}-10^{7}$ electrons and durations in the 200 fs-range (FWHM) [116] are produced, 
with capability for sub-20fs (FWHM) pulse durations with additional bunching elements [115, 342]. While MeV RF-guns are well suited for ultrafast electron diffraction [117], their implementation in imaging beamlines is rather challenging, and their transverse beam emittance is intrinsically limited by the employed photocathode geometry. The effective temporal resolution of those instruments is currently limited by the timing jitter between the RF-field and the sample exciting laser to about $60-70 \mathrm{fs}$ (FWHM) [116, 342]. Also, the acceleration scheme induces a large relative energy spread $\Delta E / E$ of about $10^{-4}-10^{-3}$.

An alternative approach for generating high-charge ultrafast electron pulses is the laserplasma acceleration of electrons created in a nitrogen gas jet by single-cycle optical pulses of $1-10 \mathrm{~mJ}$ energy at a kilohertz repetition rate. In only a few years, the available pulse charge was improved from $6.25 \cdot 10^{6}$ electrons at about $100 \mathrm{keV}$ (total energy spread: $20 \mathrm{keV}$ ) [343] to $1.5 \cdot 10^{8}$ electrons (kinetic energy distribution: $0.5-6 \mathrm{MeV}$ ) [344, 345]. Simulations indicate initial pulse durations down to $10 \mathrm{fs}$ with a reasonable beam divergence of $90 \mathrm{mrad}$ [345]. Future ultrafast diffraction applications will require extensive spatial and spectral filtering of the beam, as well as means to limit pulse dispersion and Coulomb repulsion.

\section{Low-emittance/high-coherence pulses for stroboscopic studies}

The main source of non-reversible phase space deterioration in high-charge electron pulses is Coulomb repulsion during propagation. Instead of striving for higher acceleration and final electron kinetic energy to lessen its impact, the application of space-charge free electron pulses in the single-electron limit can be desirable [25, 32, 110, 146, 198]. For planar photocathodes, such an emission regime is obtained by lowering the photoemission laser power and pulse charge to a level that does not show phase space degradation. Notably, even electron pulses of $10^{3}$ electrons emitted from large areas ( $\mu \mathrm{m}$-sized) into high static acceleration fields can approach the "single-electron" limit at a close sample with down to $100 \mathrm{fs}$ pulse durations (FWHM) [201, 214]. Phase-locked low-power RFcavities were applied for the compression of dispersed single-electron pulses from $350 \mathrm{fs}$ down to $28 \mathrm{fs}$ (FWHM) (associated with a spectral broadening from $0.4 \mathrm{eV}$ to $7.3 \mathrm{eV}$ ) [346]. Decreasing the emission area also lowers the acceptable number of electrons for space charge free operation, down to the minimum focal spot size of optical light pulses [201]. As a consequence, further enhancement of single-electron pulses requires nanoscale photocathodes. 
As applied in this thesis, the prototypical nanoscale electron emitter employs localized photoemission from sharp metal tips [150-152] which combine a $10-100 \mathrm{~nm}$ electron source size with high initial acceleration fields of about $1 \mathrm{~V} / \mathrm{nm}$. The electron emission can be easily confined to the $10 \mathrm{fs}$-scale, but implementation in an active lens assembly-as required for flexible microscopy applications-has only recently been characterized theoretically [212, 234] and in experiment [33, 140, 174, 206, 347, 348]. Further studies could demonstrate the superior transverse [157] and longitudinal [29, 182-184, 215] electron beam properties probed at a close distance.

Chapter 4 presented the first implementation of a laser-driven Schottky field emitter [158, 349] in ultrafast TEM, enabling a previously unachievable peak-brightness of ultrashort single-electron pulses.

\subsubsection{Considerations for future UTEM instruments}

The progress in conventional TEM over the last decades procured electron sources aiming for the highest possible brightness by maximizing the transverse beam coherence and minimizing the beam's energy spread. Both are achieved by employing nanoscale tipshaped field emitters. In order to transfer the wealth of well-established TEM techniques to the ultrafast timescale, laser-triggered field emitters display most promising properties (cf. Fig. 4.7). In contrast, large-area photocathodes are particularly useful for applications that require only minor transverse beam coherence but benefit from high pulse charges.

While Ch. 4 constitutes a leap forward for coherent beam applications in UTEM, future development will warrant further improvement of the beam quality in terms of transverse coherence, pulse durations and beam current, which is discussed in the following. The underlying central question is what the emitter should be designed for: either optimized single-electron pulse operation or the flexibility for upscaling the beam current.

Optimized nanoscale electron emitters If single-electron pulses are the central design objective, an emitter with lowest possible transverse emittance is desirable. That would require a reduction of the initial transverse momentum spread of the electrons or further reduction of the emitter size. The minimum intrinsic kinetic energy distribution of the photoelectron beam in Ch. 4 is $0.6 \mathrm{eV}$, which includes the overall short-term stability of the instrument. Calculations indicate the possibility to decrease the energy spread of the laser-driven Schottky field emitter [158], which is ultimately limited by the width of the 
Fermi-Dirac distribution $\Delta E_{\text {thermal }} \approx 2 k_{\mathrm{B}} T \approx 260 \mathrm{meV}$ at $T=1500 \mathrm{~K}$ and employed laser spectral bandwidth of about $60 \mathrm{meV}$ for 30 -fs laser pulses at $400-\mathrm{nm}$ central wavelength (cf. Fig. 3.7). Furthermore, sharper metal tips $\left(r_{0}<10 \mathrm{~nm}\right)$ feature a smaller effective electron source size and improved beam coherence, but were so far only capable of producing low electron yields in single-photon photoemission, e.g. $N_{e}=0.03$ electron per pulse [157]. Further development of tunable tip-based ultrafast electron sources is needed, paving the way towards point-source-like monochromatic emission characteristics. Despite the enormous advantages of single-electron pulses without Coulomb repulsion, larger tip emitters as used in this thesis with $r_{0} \sim 100 \mathrm{~nm}$ have the advantage of a high stability and allow for easy upscaling of the electron current.

For classical photoemitters, the shot-to-shot distribution of the electron number is given by Poisson statistics. A mean of $\left\langle N_{e}\right\rangle=1$ results in a probability $P(N>1)=26 \%$ of finding at least 2 electrons in an individual pulse, and only for $\left\langle N_{e}\right\rangle=0.15, P(N>1)$ drops to $1 \%$. To avoid these effects, a photon-triggered single-electron source would be needed, as already demonstrated in tunneling currents from single quantum dots [350].

Photoemission from nanoscale tips aims for the ultimate control of the electron's phase space, but so far does not treat the electron spin degree of freedom. New materials, as demonstrated for planar photocathodes [334], might yield spin-polarized electron beams.

Gun geometry The Göttingen UTEM instrument is based on a custom modified JEOL JEM-2100F Schottky field emission TEM. In continuous operation of the Schottky emitter, the beam brightness is optimized by radially cutting the transverse electron phase space distribution multiple times, thus reducing the beam current from $\sim 100 \mu \mathrm{A}$ to the $\mathrm{pA}-\mathrm{nA}$ range. The electron transmission from the tip emitter to the sample is drastically increased by individually adjusting the voltages applied to the electrostatic gun electrodes (cf. Ch. 4). Additionally, the original relatively blunt tip $\left(r_{0} \sim 430 \mathrm{~nm}\right)$ was replaced to improve the beam emittance at constant currents. These tailored operation conditions illustrate the opportunities of a customized ultrafast electron gun design. In order to reduce the impact of space charge and dispersion of the electron pulse, high acceleration fields should be applied. Simultaneously, the overall gun transmission must be optimized to achieve high beam currents at space charge-free operation. Finally, the correction of temporal aberrations should be considered (e.g. off-axis electron trajectories accumulate a small relative time delay). 

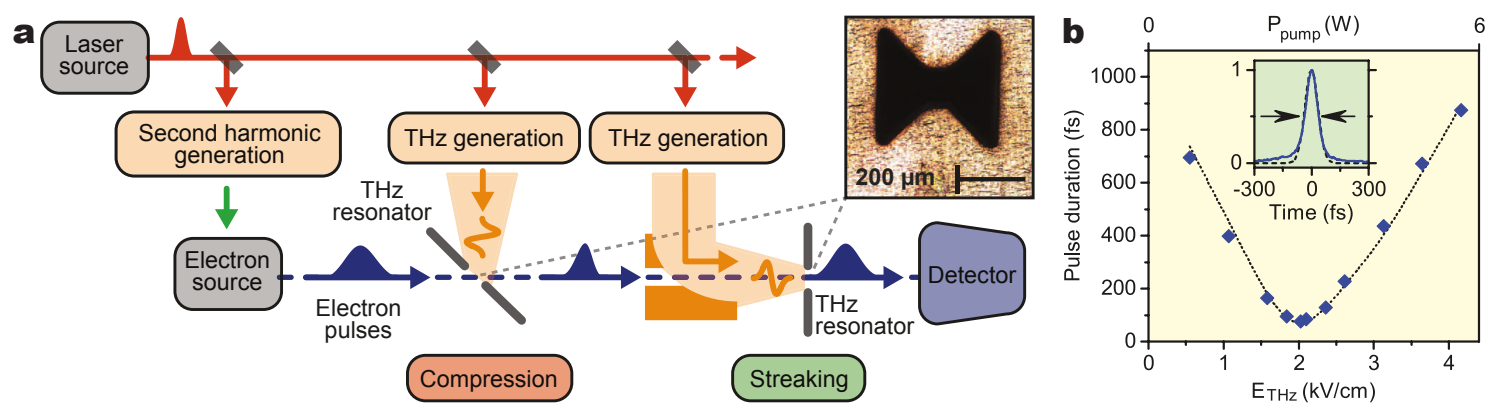

Figure 7.2: Electron pulse compression using THz-fields. (a) Schematic experimental setup with synchronized photoelectron pulse generation (930-fs initital duration) and two THz butterfly resonator structures for pulse compression and streaking. (b) Electron pulse duration (FWHM) at the streaking stage for varying incident $\mathrm{THz}$ fields used for compression (inset: shortest measured pulse profile of $75 \mathrm{fs}$ ). From [229]. Reprinted with permission from AAAS.

Shaping \& compression of electron pulses Ultrashort pulse generation by linear photoemission adds a large flexibility to freely structure the emitted electron density on a sub-ps to s timescale. Such a capability is especially useful if ns-processes, like magnetic vortex gyration [351] and skyrmion dynamics [352] are of interest, allowing for three orders of magnitude higher beam currents at fixed repetition rate without beam degradation.

In ultrafast electron diffraction setups, the use of RF-gun and RF-compressor technology allows for a tailoring of the longitudinal electron phase space on the fs- to ns timescales. The same concept was employed in early UEM implementations [124] and is also proposed for future UTEM designs using high-charge electron pulses from a planar photocathode $[119,120]$. In a similar manner, RF-compression could significantly improve the peak brightness of linearly chirped electron pulses from field emitter photocathodes (cf. Fig. 4.5). The high required stability of RF-cavities in a TEM environment has recently been demonstrated by transverse deflection and chopping of a continuous low-emittance electron beam $[122,126]$ and will enable novel applications when used in a time-of-flight type energy spectrometer [230, 336]. Nevertheless, RF-technology is challenging to be implemented and is currently still limited to a 30-fs laser/RF-phase synchronization for high-power cavities. A promising alternative is the phase space manipulation by phase-locked optical fields.

Optical (THz-) control of phase space density in the point-particle limit Optical bunching of electron pulses requires intense oscillatory fields with a period longer than the electron pulse duration (200 fs -1 ps for the Göttingen UTEM). Here, ultrashort terahertz 

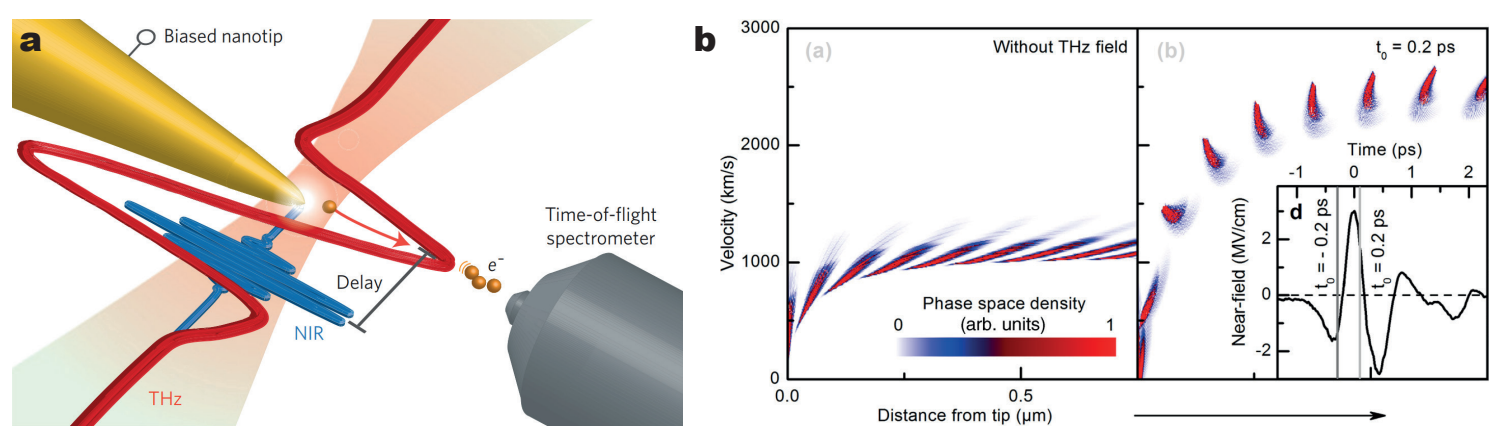

Figure 7.3: (a) Ultrashort terahertz (red) and $800 \mathrm{~nm}$ near-infrared (blue) pulses are focused onto a metallic nanotip with variable time-delay $t_{0}$ [231]. (b) Time evolution of the phase space density distribution without THz field (left) and for relative time delay of $t_{0}=0.2 \mathrm{ps}$ (right) (snapshots taken with time differences of $50 \mathrm{fs}$ ). Note the negative pre-chirping and self-compression of the phase space density [355]. (a) Adapted by permission from Springer Customer Service Centre GmbH: Springer Nature, Nature Physics, [231], @ Macmillan Publishers Limited (2014). (b) Reprinted figure with permission from [355] Copyright 2017 by the American Physical Society.

pulses deliver the highest available field amplitudes, e.g. up to $13 \mathrm{MV} / \mathrm{cm}$ in free space at a $190 \mathrm{kHz}$ repetition rate [353]. Recent experiments could demonstrate the compression of $930 \mathrm{fs}$ (FWHM) single-electron pulses by a factor of 12, down to $75 \mathrm{fs}$ (FWHM) in a $200 \mu \mathrm{m}$-gap metal resonator (cf. Fig. 7.2) [229]. The compressed electron pulses can be shorter in time than the initial photoemission laser pulse by a broadening of the kinetic energy distribution, conserving the longitudinal phase space volume according to Liouville's theorem [354].

A novel concept by Wimmer et al. discusses the control of photoemitted electrons with an additional terahertz pulse directly at the field emission tip (cf. Fig. 7.3) [231]. By adjusting the relative timing of the optical photoemission pulse and the terahertz field, the longitudinal phase space distribution can be modified. Notably, in such a geometry Liouville's theorem does not apply due to non-conservative forces acting on the electrons in the spatially and temporally inhomogeneous terahertz near-field. Hereby, elaborate phase space manipulations are feasible, like negative pre-chirping or monochromatization of the electron pulses [355]. 


\subsection{Quantum coherent control of free-electron beams}

Chapter 5 reported on the quantum-coherent optical phase-modulation of free-electron wavefunctions by intense optical near-fields. The essential enabling technology is the implementation of an ultrafast low-emittance electron gun. Nanometer-localized probing of the inhomogeneous optical near-field of a nanoscopic gold tip allows for constant amplitude interactions. The optical near-field imprints a sinusoidal phase-modulation onto the traversing single-electron wavefunctions in time, which corresponds to the formation of multiple spectral sidebands spaced by the photon energy $\hbar$. The optical-field strength dependent spectra reveal multi-level Rabi oscillations in the sideband populations, that unambiguously demonstrate the formation of a quantum coherent superposition of the associated free-electron momentum states. The coherent electron-light scattering is explained in terms of transitions and multi-path interference in an infinite $N$-level energy ladder, centered at the initial electron energy $E_{0}$. Finally, the Wigner function of such phasemodulated electrons depicts the reshaping of the longitudinal density to an attosecond electron pulse train.

The following chapter will briefly summarize the recent impact of this presented work, establishing the first elements of the new field of free-electron quantum optics. Furthermore, an outlook to future applications in ultrafast TEM is given.

\subsubsection{Coherent control of free-electron wave functions by light}

The concept of optical phase modulations can be extended to coherent control schemes using multiple interactions $[236,356]$. For example, two individual light fields will phase modulate the electrons wavefunction to form (not regarding propagation effects):

$$
\psi(z, t) \propto \psi_{0}(z, t) \cdot \exp \left(-2 i\left|g_{1}\right| \sin \left(\frac{\omega_{1}}{v_{e}} z\right)-2 i\left|g_{2}\right| \sin \left(\frac{\omega_{2}}{v_{e}} z-\varphi\right)\right)
$$

with coupling constants $g_{1 / 2}$, light frequencies $\omega_{1 / 2}$ and relative phase $\varphi$. Recent work (with the current author's contribution) was able to implement two of such coherent control schemes (cf. Fig. 7.4):

- In a Ramsey-type interferometer, the electron wavefunctions interact with two spatially separated optical near-fields, which can be individually addressed in the amplitudes $g_{1 / 2}$ and phase $\Delta \varphi$ by tailoring the polarization state of the far-field 

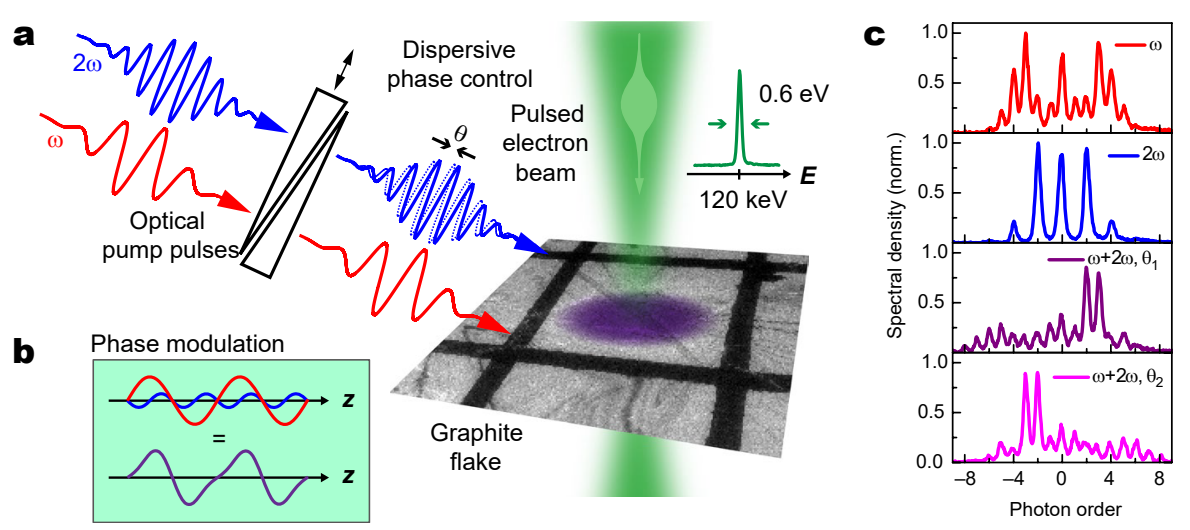

Figure 7.4: Coherent control of free-electron beams. (a) Phase-locked two-color IELS interactions enable (b) non-sinosoidal phase modulations of the passing electron wavefunction, (c) yielding strongly asymmetric final kinetic energy spectra. Figure adapted from Ref. [356], ( ) Macmillan Publishers Limited, part of Springer Nature (2017).

illumination [236]. A change in the relative phase allows for either enhancing or canceling of the first optical phase modulation, which constitutes a remarkable demonstration of the coherence of the scattering process. These results pave the way for novel type of experiments, like the study of dephasing mechanisms in electron-matter interaction.

- Simultaneous multi-color interactions with two $400 \mathrm{~nm}$ and $800 \mathrm{~nm}$ light-fields enable non-sinusoidal phase modulations, facilitating the formation of highly asymmetric populations in the kinetic momentum state superpositions (7.4) [356]. This experiment illustrates the capability for shaping electron wavefunction in the longitudinal direction by tailored optical fields.

The work by Priebe et al. [356] also introduced a novel quantum state tomography technique termed "SQUIRRELS" ("spectral quantum interference for the regularized reconstruction of free-electron states"), retrieving the density matrix of the longitudinal phase modulated free-electron ensemble.

\subsubsection{Generation of attosecond electron pulse trains}

The study of ultrafast electron dynamics on the atomic to molecular level requires an attosecond temporal resolution, as demonstrated in optical science [357]. In high harmonic generation $(\mathrm{HHG})$ bound electronic states are phase-modulated by light to generate bursts of attosecond ultraviolet photon pulses [23]. A similar concept is applied in free- 

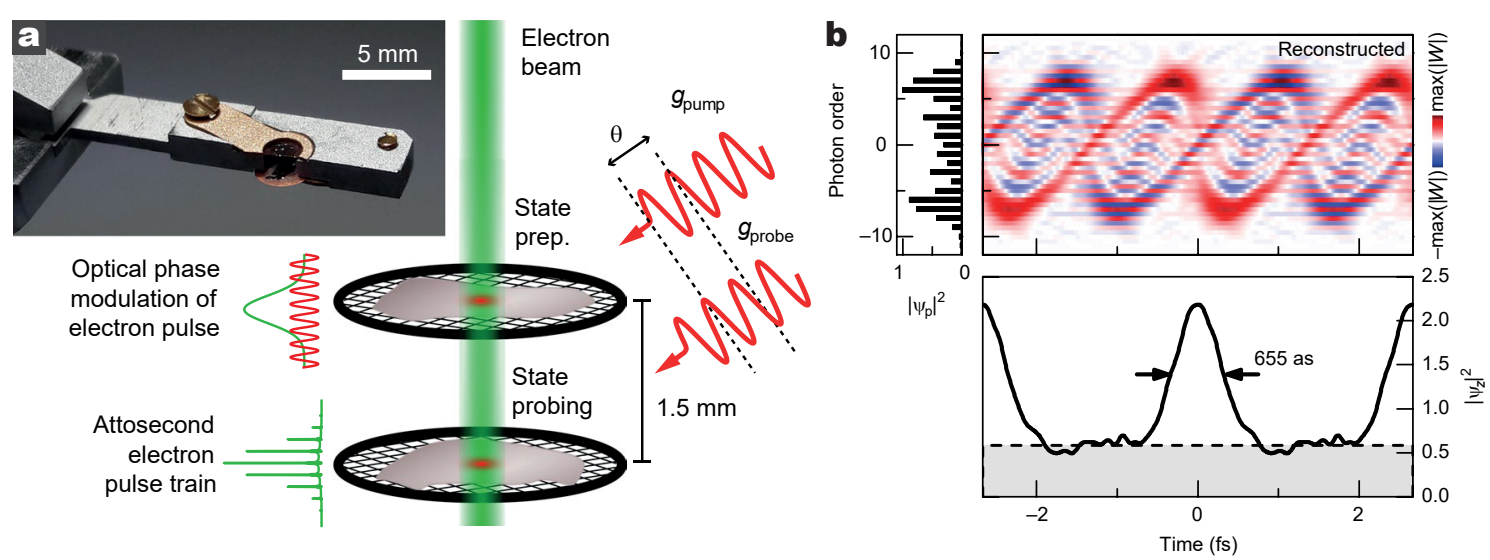

Figure 7.5: Generation and reconstruction of attosecond electron pulse trains in UTEM. (a) Schematic experimental setup for state preparation and probing by coherent optical phase modulation (top-left: double-sided sample holder). (b) The quantum state after dispersive propagation is reconstructed in terms of its density matrix. The corresponding Wigner function displays density spikes of 655 as in temporal projection. Figure adapted from Ref. [356], () Macmillan Publishers Limited, part of Springer Nature (2017).

electron laser science [358], producing electron microbunches by optical phase-dependent accelerations of relativistic high-charge free-electron pulses [359, 360].

The compression of electron pulses to attosecond pulse trains was proposed using ponderomotive forces acting on point-like particles in co-moving monochromatic [263, 361] or phase-matched bi-chromatic [362] optical intensity gratings. Extending this concept, the calculations presented in $\mathrm{Ch} .5$ predict the evolution of longitudinal density modulation of free-electron beams after quantum coherent optical phase-modulation. As a consequence, each individual electron wavefunction evolves short attosecond spikes in its density, while roughly maintaining its overall temporal envelope. In an ensemble average, an attosecond electron pulses train is formed.

Recent work in the Göttingen UTEM project [356] achieved the first demonstration of such an attosecond electron pulse train within an ultrafast TEM (cf. Fig. 7.5). The full reconstruction of the longitudinal electron quantum state reveals electron density modulations with a temporal width of only 655 as (FWHM). In parallel work, Morimoto et al. could produce electron pulse trains of 810 as temporal peak width (FWHM) in an ultrafast electron diffraction geometry [363] and Kozák et al. showed the preparation by using bi-chromatic intense light-fields at a 1-kHz repetition rate in free-space [364].

The availability of attosecond electron pulse trains in ultrafast TEM will enable novel avenues in the study of light-driven electron dynamics in solids with unprecedented 
temporal and spatial resolution. Further development is needed to generate isolated attosecond electron pulses and to reduce their temporal width down to the theoretically limit given by Heisenberg's uncertainty principle. ${ }^{1}$

\subsubsection{A three-dimensional all-optical electron phase plate}

In conventional TEM, the coherent transverse phase modulation of electron wavefunctions is an ubiquitous phenomenon, which is applied for the development of elaborate phase masks, coherently shaping electron beams in the transverse direction (cf. Sec. 2.1). The Aharonov-Bohm equation (Eq. 2.1) gives the phase shift an electron acquires while passing static electromagnetic fields.

Quantum coherent optical phase modulation now allows for a generalization of this concept by imprinting time-varying phase shifts onto free-electron beams (cf. 7.6). Accessing the longitudinal electron phase space enables manipulation of electron beams in energy and time. Novel capabilities accessible with such a time-dependent phase-modulator are exemplified in the previous sections.

The high flexibility of controlling free electrons with externally shaped light fields [237, 365], was explored in first applications using intense ns-laser pulses, e.g. by observation of the Kapitza-Dirac effect [257] and recently in cavity enhanced continuous laser fields for constructing an optical Zernike phase-plate $[366,367]$. Notably, the inelastic electron-light scattering described in Ch. 5 is generally associated with a momentum transfer in three dimensions [368] (cf. Fig. 7.6) and was proposed for the generation of tailored electron beam states, like the transfer of orbital angular momentum and the light-induced generation of electron vortex beams [236, 369].

\subsection{Probing ultrafast nanoscale dynamics in UTEM}

Chapter 6 reports on the quantitative diffractive probing of structural dynamics at the edge of a laser-excited single crystalline graphite membrane with $28-\mathrm{nm} / 700$-fs spatio-temporal resolution. Ultrafast convergent beam electron diffraction (U-CBED) patterns are recorded, simultaneously mapping the full diffraction rocking curves of multiple Bragg scattering conditions. The time-dependent local unit cell deformation is analyzed by retrieving

\footnotetext{
${ }^{1}$ E.g., a single Gaussian shaped electron wave packet, e.g. with an energy width of $20 \mathrm{eV}$ (FWHM) has Fourier-limited temporal spread of 91 as (FWHM) (cf. Eq. 3.18).
} 


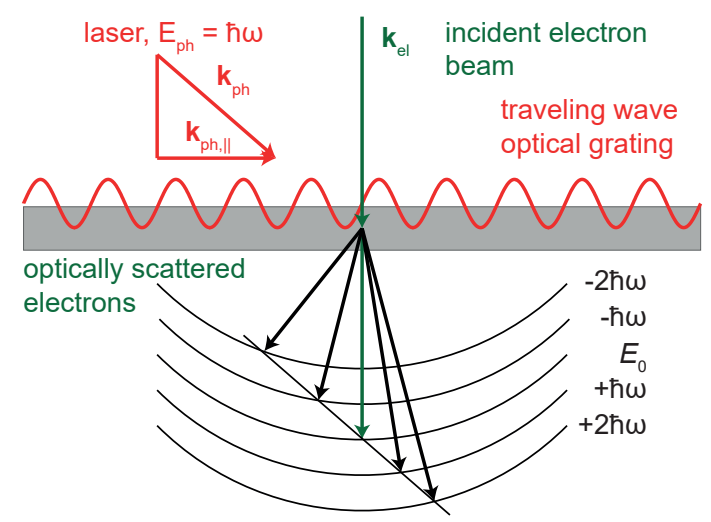

Figure 7.6: Transverse effects in IELS. The coherent electron-light scattering is generally associated with a three-dimensional momentum transfer. The directional linear momentum of a traveling wave optical grating will deflect specific photon orders accordingly.

components of the deformation gradient tensor, disentangling the relevant mechanical deformation modes.

Specifically, the top face of the graphite membrane is optically excited with the intensity being modulated by a near-field interference structure at the edge. The decay of the initial non-thermal phonon distribution and the increase of the local lattice temperature is evaluated from the study of the time-dependent Debye-Waller behavior. The ultrafast buildup of thermal stress leads to an out-of-plane lattice expansion and excitation of a coherent membrane breathing mode. Due to the symmetry breaking at the edge, an in-plane propagating expansive shock wave is launched in the top face of the membrane. Hereby, a membrane shearing mode is locally excited with a characteristic phase offset, that linearly increases with distance to the edge. Finally, the particularly pronounced inhomogeneous broadening of the Bragg line-profiles is compared to numerical simulations, which reveal complex depth-dependent three-dimensional strain dynamics.

\subsubsection{Time-domain access to nanophononic systems}

The field of nanophononics is striving for tailored phonon properties and interactions in nanoscale systems, that are coupled to various external degrees of freedom [79] (cf. Fig. 7.7 and Sec. 6.1). At typical sound velocities, nano-localized phonon modes occur on the giga- to terahertz frequency range, illustrating the need for simultaneous high spatial and temporal resolution. Optical spectroscopy routinely allows for meV-spectral [79] or fs-time-domain studies $[315,316]$ of coherent and incoherent phonon dynamics. However, its spatial resolution is intrinsically limited to the $\mu \mathrm{m}$-scale. In contrast, monochromated TEM recently achieved vibrational spectroscopy with simultaneous $10-\mathrm{meV}$ spectral and 

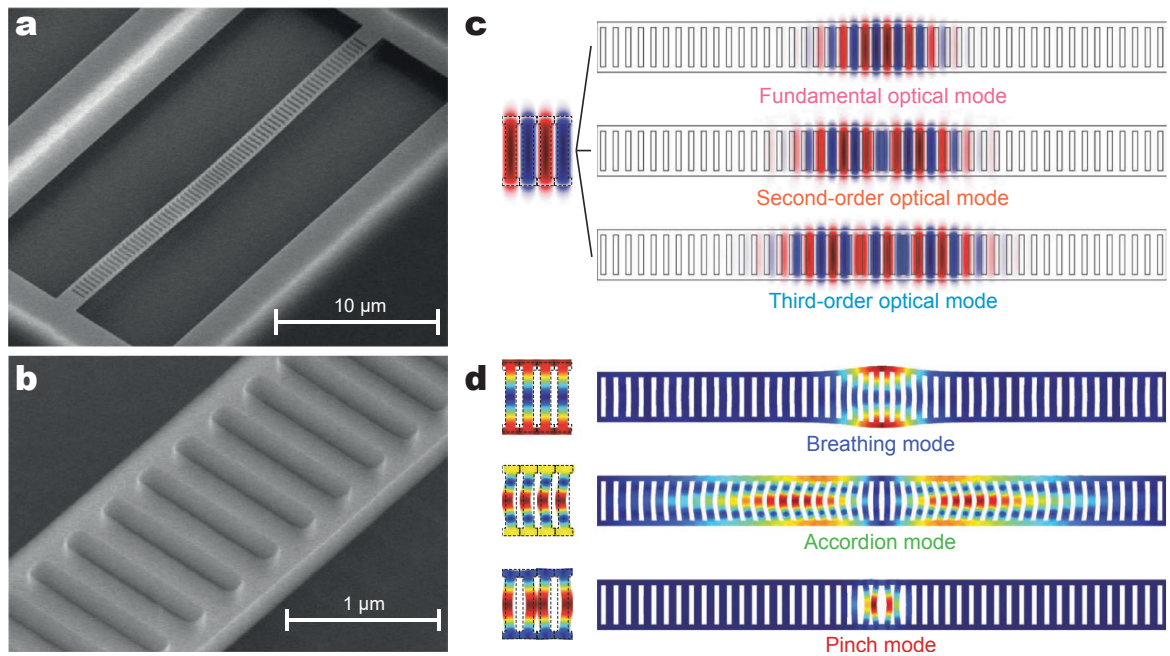

Figure 7.7: Nanoscale optomechanical crystal. Design of photonic-phononic resonator $((\mathrm{a}, \mathrm{b})$ : SEM images) that exhibits coupled optical and mechanical eigenmodes when illuminated with monochromatic light (c,d). Adapted by permission from Springer Customer Service Centre GmbH: Springer Nature, Nature, [281], (C) Macmillan Publishers Limited (2009).

2- $\AA$ spatial resolution $[15,95]$, though having no time-domain access to coherent phonon excitations.

Here, U-CBED adds a unique capability to the toolset of nanophononics by giving quantitative three-dimensional access to optically-driven coherent lattice dynamics with nanometer spatial and femtosecond temporal resolution. Furthermore, as presented in Ch. 6, UTEM offers a tremendous experimental flexibility to

- characterize the spatial distribution of optical sample excitations by PINEM,

- track the fs- to ps-thermalization of the initial anisotrospic phonon distribution,

- measure the local lattice temperature with nm-spatial and fs-temporal resolution,

- analyze the deformation gradient tensor that quantitatively disentangles the contributions of specific phonon-modes and their coupling and

- map nanoscale phonon transport and dissipation on ps- to ns-timescales.

Future research directions for UTEM include the rapidly advancing field of cavity optomechanics (cf. Fig. 7.7) [273], e.g. harnessing the quantum-coherent coupling of mechanical and optical modes [274]. 


\subsubsection{Methods for studying ultrafast nanoscale dynamics}

A central aspect of the thesis at hand is the development of new instrumental capabilities in ultrafast electron microscopy for the study of ultrafast nanoscale dynamics. However, assessing the future prospects of the UTEM methodology requires a comprehensive comparison to other ultrafast high-resolution probing techniques, which is presented in the following and summarized in Fig. 7.8.

The most relevant parameters to assess the capabilities of ultrafast probing techniques include (i) real-space (ii) k-space (iii) spectroscopic and (iv) temporal resolution. Furthermore, the couplings to the sample and corresponding acquired information depend on the (v) type of particle or radiation and their (vi) respective wavelengths. The (vii) coherence properties determine the usefulness of holography or phase contrast imaging. Finally, it must be distinguished between techniques that use (iix) near-field or far-field probing, being a key aspect for flexibility and required sample geometry and their (ix) sensitivity to surfaces, thin film samples or bulk materials.

\section{Photon based methods}

Optical spectroscopy and microscopy using visible light provide for an excellent few-fs temporal and few-meV spectral resolution. Therefore, optical far-field probing methods are ideally suited for the study of ultrafast electronic phenomena in homogeneous condensed matter [317] and gas-phase systems [370] at the femtosecond time scale. Nanoscale local probing of electronic states is provided by scanning near-field optical microscopy [371] and THz-field gated scanning tunneling microscopy [27]. Recently, table-top high-harmonic sources, which can readily reach attosecond pulse durations [23, 244], could achieve a real-space resolution on the 10-nm scale by coherent diffractive imaging (CDI) [372, 373] with tailored contrast mechanisms, e.g. to out-of-plane magnetization [374]. Yet, direct far-field optical probing of lattice dynamics requires photon wavelengths matched to the atomic distances and is so far only achieved in large-scale synchrotron or FEL facilities $[375,376]$. Atomic scale reciprocal space and a 10-100-nm real space resolution were shown by X-ray nanodiffraction [377-379] or lensless imaging approaches [176, 380].

Notably, optical methods benefit from almost fully coherent light sources that enable diffraction-limited spatial resolution and bandwidth-limited ultrashort pulses in a routinely manner. Coherent beam techniques like in-line or off-axis holography, interferometry, high-quality phase contrast imaging and advanced beam shaping contribute to the powerful 
toolset of optical science. On the downside, lab-scale ultrafast coherent light sources of sub-nm wavelength are not available, and the low scattering cross-sections and high penetration depths render the study of nanoscale volumes increasingly difficult [381].

\section{Electron probing}

Ultrashort electron pulses are ideally suited as probes for ultrafast lattice dynamics due to their intrinsically short wavelength, high scattering cross-sections and technological synergy with the well-developed field of electron microscopy. Ultrafast far-field electron diffraction in transmission (UED) [25, 114] or reflection (U-RHEED, U-LEED) [78, 174] routinely achieves down to 100-fs temporal [201, 214] and sub- $\AA$ resolution in k-space [77], typically probing sample areas on the $10-100 \mu \mathrm{m}$ scale. Further strategies for improved electron source quality were discussed in Sec. 7.1. One alternative approach is to use localized photoelectron emission from clean surfaces. Time and angle resolved photoemission spectroscopy (trARPES) [382, 383] achieves laser bandwidth-limited temporal and spectral resolution and time-resolved photoemission electron microscopy (TR-PEEM) [384] gives a below 100-nm spatial resolution, e.g. of local sample magnetization [385]. For gas targets, laser-induced electron diffraction (LIED) [386, 387] yields a sub-optical cycle timing-accuracy of inertial molecular dynamics. Another promising approach to probe electronic dynamics and optical near-fields is by lens-less projection to the far-field in ultrafast point-projection microscopy (PPM) [29, 182-184] with a few 10-nm spatial and fs temporal resolution.

\section{Coherent ultrafast TEM}

Each of the ultrafast electron probing techniques described above demonstrate tailored capabilities for either excellent k-space or real space resolution, combined with high sensitivity to specific sample properties. Usually, a high temporal resolution is achievable, but strongly depending on the experimental geometry. The primary achievement of ultrafast transmission electron microscopy is a combination of either excellent $\mathrm{k}$-space or real space probing, with simultaneous sub-ps temporal and about $1 \mathrm{eV}$ energy resolution. State-of-the-art TEM optics enable a highly flexible investigation of thin film samples in diffraction, imaging or spectroscopy with sensitivity to electronic, magnetic and lattice degrees-of-freedom. 
The instrumental development presented in this thesis further expands the capabilities of UTEM, now spanning orders of magnitude in real-space ( $1 \mathrm{~nm}$ to $1 \mathrm{~mm}$ ) and k-space (sub $1 \AA^{-1}$ to $1 \mu \mathrm{m}^{-1}$ ) for the stroboscopic probing of ultrafast dynamics in condensed matter with a temporal resolution of down to $200 \mathrm{fs}$. Notably, the strongly enhanced coherence properties of electron beams generated from nanoscale photocathodes have not been fully harnessed yet. Access to holography, interferometry, advanced beam shaping and high-resolution phase contrast imaging will drastically expand the addressable scientific questions and sample systems of ultrafast TEM, making it one of the most universal tools for the study of ultrafast nanoscale dynamics (cf. Fig. 7.8).

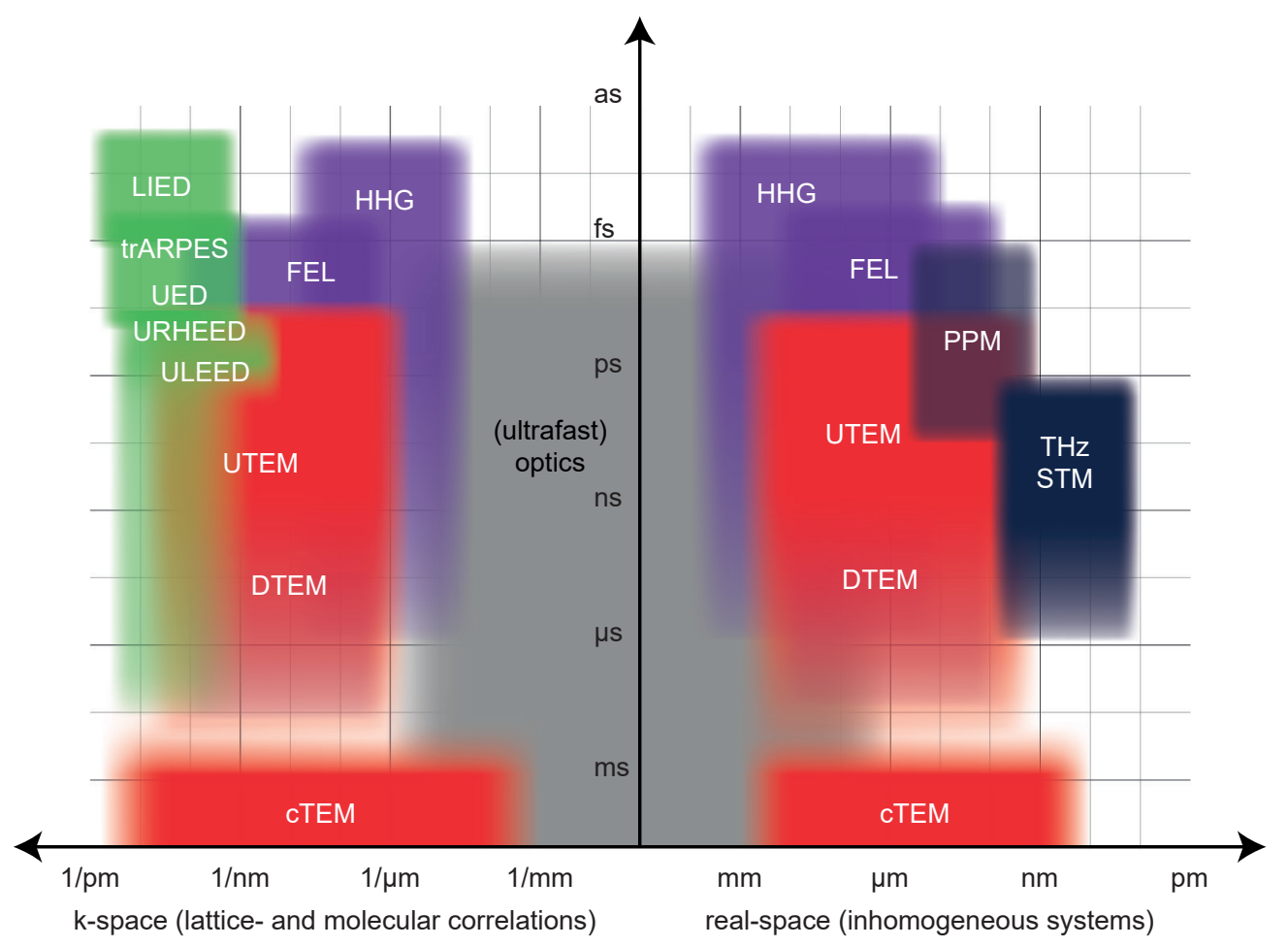

Figure 7.8: Time and length scales addressed by fast probing techniques. A variety of ultrafast techniques aims for a particular high resolution in real or reciprocal space and tailored sensitivity to specific degrees-of-freedom. Ultrafast TEM features a broad range of applications and explores systems on many orders of magnitude in time, reciprocal space and real space simultaneously. 


\subsection{Conclusion \& further perspectives}

In this thesis, a novel ultrafast TEM instrument was developed, for the first time using ultrashort coherent electron pulses from nanoscale photocathodes. The electron pulses of unprecedented coherence properties now available in UTEM facilitate advanced imaging, diffraction and spectroscopic analysis on the femtosecond timescale, with future attosecond capability.

Furthermore, two scientific applications-enabled only by instrumental progress presented here-were explored in detail. Firstly, the coherent optical phase modulation of free-electron wavefunctions by inelastic scattering in optical near-fields was introduced, opening up a new degree of freedom for the quantum coherent control of free-electron beams. Secondly, in a prototypical experiment, the nanoscale diffractive probing of ultrafast strain dynamics with 28-nm spatial and 700-fs temporal resolution was demonstrated by establishing ultrafast convergent beam electron diffraction (U-CBED).

However, these initial applications just indicate the future possibilities of coherent ultrafast TEM. Many techniques from the highly refined toolset of conventional TEM can be proliferated to the femtosecond timescale and new physics under extreme conditions may be explored.

Increasing the capabilities of ultrafast science, coherent UTEM opens up a broad range of research directions, addressing the couplings to electronic, magnetic and lattice degreesof-freedom, as well as the coherent beam-tailoring by electromagnetic fields and their nanoscale probing (cf. Fig. 7.9).

In his famous lecture "There's Plenty of Room at the Bottom", Richard Feynman proclaimed a proliferation of electron microscopy by enhanced spatial resolving power. With the recent technological advancement, there is also a tremendous demand of pushing the combined spatio-temporal resolution available to the ultimate level. Here, advanced UTEM instruments will prove pivotal to harness the uncharted territories of ultrafast nanoscience. 


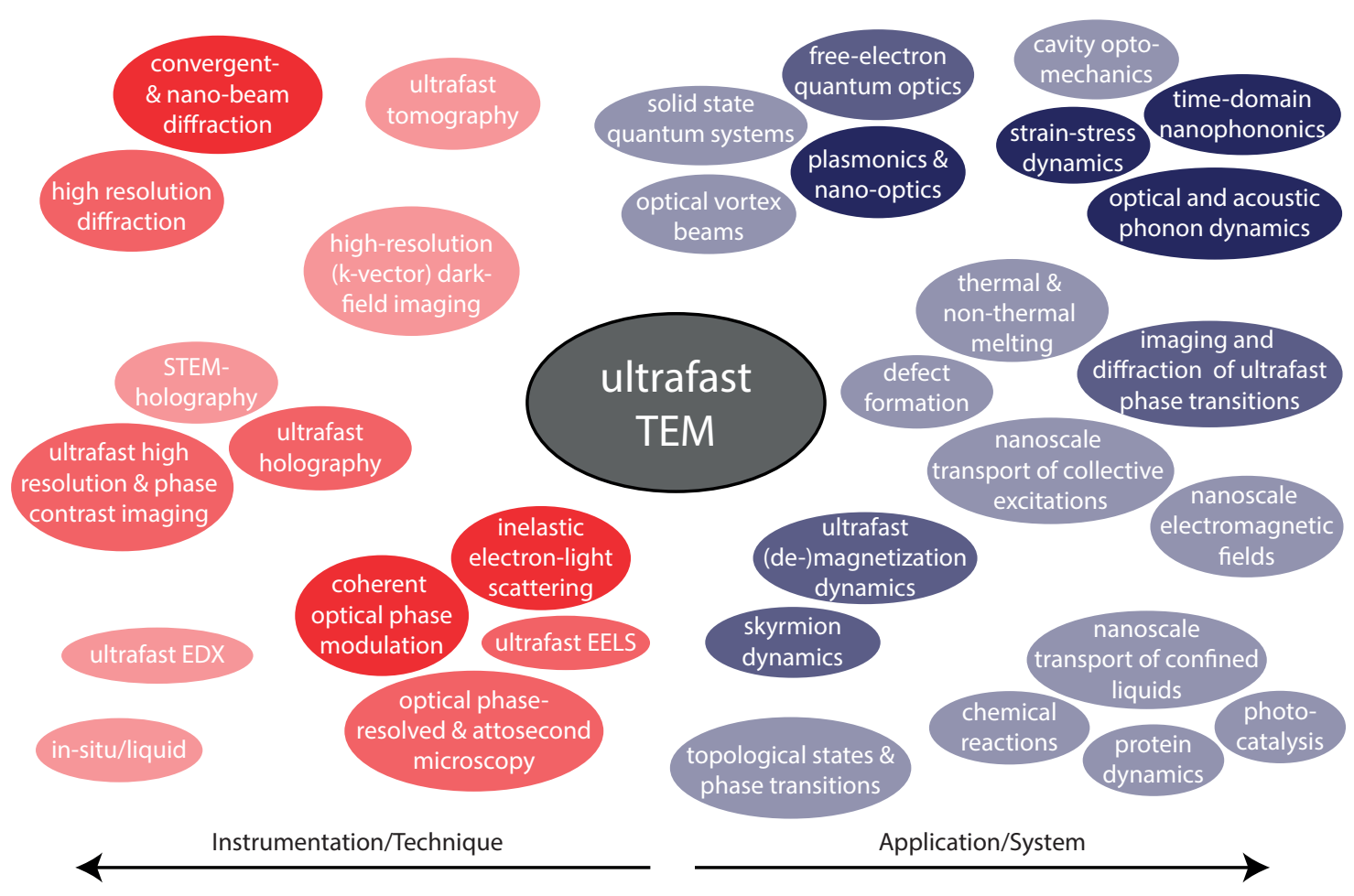

Figure 7.9: Prospects of coherent UTEM. The availability of coherent electron beams in ultrafast TEM opens up a broad range of future techniques and applications. Fainter shading indicates topics that are further outside the scope of this thesis, with many areas being mostly unexplored to the current date. 



\section{Bibliography}

[1] E. Abbe. Beiträge zur Theorie des Mikroskops und der mikroskopischen Wahrnehmung. Archiv für Mikroskopische Anatomie, 9(1):413-418, 1873. Cited on page 1 .

[2] E. Mach and P. Salcher. Photographische Fixirung der durch Projectile in der Luft eingeleiteten Vorgänge. Annalen der Physik und Chemie, 268(10):277-291, 1887. Cited on page 1.

[3] W. Friedrich, P. Knipping, and M. Laue. Interferenzerscheinungen bei Röntgenstrahlen. Annalen der Physik, 346(10):971-988, 1913. Cited on page 1.

[4] G. P. Thomson and A. Reid. Diffraction of Cathode Rays by a Thin Film. Nature, 119(3007):890-890, 1927. Cited on pages 1 and 14.

[5] M. Knoll and E. Ruska. Das Elektronenmikroskop. Zeitschrift für Physik, 78(56):318-339, 1932. Cited on pages 1 and 6 .

[6] O. Scherzer. The Theoretical Resolution Limit of the Electron Microscope. Journal of Applied Physics, 20(1):20-29, 1949. Cited on page 1.

[7] M. Haider, S. Uhlemann, E. Schwan, and H. Rose. Electron microscopy image enhanced. Nature, 392(April):768-769, 1998. Cited on page 1.

[8] P. E. Batson, N. Dellby, and O. L. Krivanek. Sub-ångstrom resolution using aberration corrected electron optics. Nature, 418(6898):617-20, 2002. Cited on page 1 .

[9] R. Erni, M. D. Rossell, C. Kisielowski, and U. Dahmen. Atomic-Resolution Imaging with a Sub-50-pm Electron Probe. Physical Review Letters, 102(9):096101, 2009. Cited on page 1. 
[10] O. L. Krivanek, M. F. Chisholm, V. Nicolosi, T. J. Pennycook, G. J. Corbin, N. Dellby, M. F. Murfitt, C. S. Own, Z. S. Szilagyi, M. P. Oxley, S. T. Pantelides, and S. J. Pennycook. Atom-by-atom structural and chemical analysis by annular dark-field electron microscopy. Nature, 464(7288):571-574, 2010. Cited on pages 1 and 11.

[11] O. Krivanek and P. Mooney. Applications of slow-scan CCD cameras in transmission electron microscopy. Ultramicroscopy, 49(1-4):95-108, 1993. Cited on page 1 .

[12] R. A. Crowther, D. J. DeRosier, and A. Klug. The Reconstruction of a ThreeDimensional Structure from Projections and its Application to Electron Microscopy. Proceedings of the Royal Society A: Mathematical, Physical and Engineering Sciences, 317(1530):319-340, 1970. Cited on page 1.

[13] K. Grünewald, P. Desai, D. C. Winkler, J. B. Heymann, D. M. Belnap, W. Baumeister, and A. C. Steven. Three-Dimensional Structure of Herpes Simplex Virus from Cryo-Electron Tomography. Science, 302(5649):1396-1398, 2003. Cited on page 1 .

[14] O. L. Krivanek, A. J. Gubbens, N. Dellby, and C. E. Meyer. Design and first applications of a post-column imaging filter. Microscopy Microanalysis Microstructures, 3(2-3):187-199, 1992. Cited on page 1.

[15] O. L. Krivanek, T. C. Lovejoy, N. Dellby, T. Aoki, R. W. Carpenter, P. Rez, E. Soignard, J. Zhu, P. E. Batson, M. J. Lagos, R. F. Egerton, and P. A. Crozier. Vibrational spectroscopy in the electron microscope. Nature, 514(7521):209-212, 2014. Cited on pages 1 and 122.

[16] A. Tonomura. Electron Holography. 2nd edition, vol. 70. Springer Series in Optical Sciences. Berlin, Heidelberg: Springer Berlin Heidelberg, 1999, 163 pages. Cited on page 1.

[17] J. Verbeeck, H. Tian, and P. Schattschneider. Production and application of electron vortex beams. Nature, 467(7313):301-304, 2010. Cited on pages 1, 9 and 58.

[18] A. L. Schawlow and C. H. Townes. Infrared and Optical Masers. Physical Review, 112(6):1940-1949, 1958. Cited on page 1.

[19] T. H. Maiman. Stimulated Optical Radiation in Ruby. Nature, 187(4736):493-494, 1960. Cited on page 1 . 
[20] H. W. Mocker and R. J. Collins. Mode Competition and Self-Locking Effects in a Q-Switched Ruby Laser. Applied Physics Letters, 7(10):270-273, 1965. Cited on page 1.

[21] A. J. DeMaria, D. A. Stetser, and H. Heynau. Self mode-locking of lasers with saturable absorbers. Applied Physics Letters, 8(7):174-176, 1966. Cited on page 1.

[22] D. E. Spence, P. N. Kean, and W. Sibbett. 60-fsec pulse generation from a selfmode-locked Ti:sapphire laser. Optics Letters, 16(1):42, 1991. Cited on page 1.

[23] P. B. Corkum and F. Krausz. Attosecond science. Nature Physics, 3(6):381-387, 2007. Cited on pages 1,118 and 123.

[24] R. D. Miller. Mapping Atomic Motions with Ultrabright Electrons: The Chemists' Gedanken Experiment Enters the Lab Frame. Annual Review of Physical Chemistry, 65(1):583-604, 2014. Cited on page 2.

[25] A. H. Zewail. 4D ultrafast electron diffraction, crystallography, and microscopy. Annual Review of Physical Chemistry, 57(1):65-103, 2006. Cited on pages 2, 5, 8, 40, 112 and 124.

[26] A. M. Lindenberg, J. Larsson, K. Sokolowski-Tinten, K. J. Gaffney, C. Blome, O. Synnergren, J. Sheppard, C. Caleman, A. G. MacPhee, D. Weinstein, D. P. Lowney, T. K. Allison, T. Matthews, R. W. Falcone, A. L. Cavalieri, D. M. Fritz, S. H. Lee, P. H. Bucksbaum, D. A. Reis, J. Rudati, P. H. Fuoss, C. C. Kao, D. P. Siddons, R. Pahl, J. Als-Nielsen, S. Duesterer, R. Ischebeck, H. Schlarb, H. SchulteSchrepping, T. Tschentscher, J. Schneider, D. von der Linde, O. Hignette, F. Sette, H. N. Chapman, R. W. Lee, T. N. Hansen, S. Techert, J. S. Wark, M. Bergh, G. Huldt, D. van der Spoel, N. Timneanu, J. Hajdu, R. A. Akre, E. Bong, P. Krejcik, J. Arthur, S. Brennan, K. Luening, and J. B. Hastings. Atomic-Scale Visualization of Inertial Dynamics. Science, 308(5720):392-395, 2005. Cited on pages 2, 40 and 86.

[27] T. L. Cocker, D. Peller, P. Yu, J. Repp, and R. Huber. Tracking the ultrafast motion of a single molecule by femtosecond orbital imaging. Nature, 539(7628):263-267, 2016. Cited on pages 2, 40 and 123. 
[28] V. Kravtsov, R. Ulbricht, J. M. Atkin, and M. B. Raschke. Plasmonic nanofocused four-wave mixing for femtosecond near-field imaging. Nature Nanotechnology, 11(5):459-464, 2016. Cited on pages 2 and 40.

[29] M. Müller, A. Paarmann, and R. Ernstorfer. Femtosecond electrons probing currents and atomic structure in nanomaterials. Nature Communications, 5:5292, 2014. Cited on pages 2, 40, 44, 113 and 124.

[30] H. Dömer and O. Bostanjoglo. High-speed transmission electron microscope. Review of Scientific Instruments, 74(10):4369, 2003. Cited on pages 2, 5, 8 and 40.

[31] J. S. Kim, T. LaGrange, B. W. Reed, M. L. Taheri, M. R. Armstrong, W. E. King, N. D. Browning, and G. H. Campbell. Imaging of transient structures using nanosecond in situ TEM. Science, 321(5895):1472-1475, 2008. Cited on pages 2, $5,8,40$ and 86 .

[32] A. H. Zewail. Four-Dimensional Electron Microscopy. Science, 328(5975):187193, 2010. Cited on pages 2, 8, 40, 42, 86, 100 and 112.

[33] A. Feist, N. Bach, N. Rubiano da Silva, T. Danz, M. Möller, K. E. Priebe, T. Domröse, J. G. Gatzmann, S. Rost, J. Schauss, S. Strauch, R. Bormann, M. Sivis, S. Schäfer, and C. Ropers. Ultrafast transmission electron microscopy using a laser-driven field emitter: Femtosecond resolution with a high coherence electron beam. Ultramicroscopy, 176:63-73, 2017. Cited on pages 3, 86, 87, 100, 101 and 113.

[34] A. Feist, K. E. Echternkamp, J. Schauss, S. V. Yalunin, S. Schäfer, and C. Ropers. Quantum coherent optical phase modulation in an ultrafast transmission electron microscope. Nature, 521(7551):200-203, 2015. Cited on pages 3, 41, 42, 44, 49, $51,52,55,56,57,86,87,96,106$ and 107.

[35] A. Feist, N. Rubiano da Silva, W. Liang, C. Ropers, and S. Schäfer. Nanoscale diffractive probing of strain dynamics in ultrafast transmission electron microscopy. Structural Dynamics, 5(1):014302, 2018. Cited on page 3.

[36] D. B. Williams and C. B. Carter. Transmission Electron Microscopy. Boston, MA: Springer US, 2009. Cited on pages 5, 6, 8, 11 and 41.

[37] D. J. Flannigan and A. H. Zewail. 4D Electron Microscopy: Principles and Applications. Accounts of Chemical Research, 45(10):1828-1839, 2012. Cited on pages 5 and 100. 
[38] J. C. H. Spence and J. M. Zuo. Electron Microdiffraction. Boston, MA: Springer US, 1992, 388 pages. Cited on pages 6, 87, 95 and 102.

[39] L. Reimer and H. Kohl. Transmission Electron Microscopy. Vol. 36. Springer Series in Optical Sciences. New York, NY: Springer New York, 2008. Cited on pages $6,27,41$ and 44 .

[40] R. Brydson, ed. Aberration-Corrected Analytical Transmission Electron Microscopy. Chichester, UK: John Wiley \& Sons, Ltd, 2011, 296 pages. Cited on page 6.

[41] J. C. H. Spence. High-Resolution Electron Microscopy. Oxford University Press, 2013, 432 pages. Cited on pages 6, 11, 41 and 44.

[42] C. B. Carter and D. B. Williams, eds. Transmission Electron Microscopy. Cham: Springer International Publishing, 2016, 518 pages. Cited on page 6.

[43] J. M. Zuo and J. C. Spence. Advanced Transmission Electron Microscopy. New York, NY: Springer New York, 2017, 729 pages. Cited on pages 6, 10, 11, 12, 13, $31,32,33,36,86,87,88$ and 92.

[44] G. McMullan, A. Faruqi, D. Clare, and R. Henderson. Comparison of optimal performance at $300 \mathrm{keV}$ of three direct electron detectors for use in low dose electron microscopy. Ultramicroscopy, 147:156-163, 2014. Cited on page 8.

[45] H. Kiesel, A. Renz, and F. Hasselbach. Observation of Hanbury Brown-Twiss anticorrelations for free electrons. Nature, 418(July):392-394, 2002. Cited on page 8.

[46] W. E. King, G. H. Campbell, A. Frank, B. Reed, J. F. Schmerge, B. J. Siwick, B. C. Stuart, and P. M. Weber. Ultrafast electron microscopy in materials science, biology, and chemistry. Journal of Applied Physics, 97(11):111101, 2005. Cited on page 8 .

[47] N. Browning, M. Bonds, G. Campbell, J. Evans, T. LaGrange, K. Jungjohann, D. Masiel, J. McKeown, S. Mehraeen, B. Reed, and M. Santala. Recent developments in dynamic transmission electron microscopy. Current Opinion in Solid State and Materials Science, 16(1):23-30, 2012. Cited on pages 8, 40, 42 and 51.

[48] Y. Aharonov and D. Bohm. Significance of Electromagnetic Potentials in the Quantum Theory. Physical Review, 115(3):485-491, 1959. Cited on page 8. 
[49] F. Zernike. Phase contrast, a new method for the microscopic observation of transparent objects. Physica, 9(7):686-698, 1942. Cited on page 9.

[50] F. Zernike. How I Discovered Phase Contrast. Science, 121(3141):345-349, 1955. Cited on page 9.

[51] R. Danev and K. Nagayama. Transmission electron microscopy with Zernike phase plate. Ultramicroscopy, 88(4):243-252, 2001. Cited on page 9.

[52] B. J. McMorran, A. Agrawal, I. M. Anderson, A. A. Herzing, H. J. Lezec, J. J. McClelland, and J. Unguris. Electron vortex beams with high quanta of orbital angular momentum. Science, 331(6014):192-195, 2011. Cited on pages 9 and 58.

[53] L. Allen, M. W. Beijersbergen, R. J. C. Spreeuw, and J. P. Woerdman. Orbital angular momentum of light and the transformation of Laguerre-Gaussian laser modes. Physical Review A, 45(11):8185-8189, 1992. Cited on page 9.

[54] M. Uchida and A. Tonomura. Generation of electron beams carrying orbital angular momentum. Nature, 464(7289):737-739, 2010. Cited on pages 9 and 58.

[55] V. Grillo, G. C. Gazzadi, E. Mafakheri, S. Frabboni, E. Karimi, and R. W. Boyd. Holographic generation of highly twisted electron beams. Physical Review Letters, 114(3):1-5, 2015. Cited on page 9.

[56] R. Juchtmans, A. Béché, A. Abakumov, M. Batuk, and J. Verbeeck. Using electron vortex beams to determine chirality of crystals in transmission electron microscopy. Physical Review B, 91(9):094112, 2015. Cited on page 10.

[57] G. Guzzinati, A. Béché, H. Lourenço-Martins, J. Martin, M. Kociak, and J. Verbeeck. Probing the symmetry of the potential of localized surface plasmon resonances with phase-shaped electron beams. Nature Communications, 8:14999, 2017. Cited on page 10 .

[58] V. Grillo, T. R. Harvey, F. Venturi, J. S. Pierce, R. Balboni, F. Bouchard, G. Carlo Gazzadi, S. Frabboni, A. H. Tavabi, Z.-A. Li, R. E. Dunin-Borkowski, R. W. Boyd, B. J. McMorran, and E. Karimi. Observation of nanoscale magnetic fields using twisted electron beams. Nature Communications, 8(1):689, 2017. Cited on page 10 . 
[59] V. Grillo, A. H. Tavabi, E. Yucelen, P.-H. Lu, F. Venturi, H. Larocque, L. Jin, A. Savenko, G. C. Gazzadi, R. Balboni, S. Frabboni, P. Tiemeijer, R. E. DuninBorkowski, and E. Karimi. Towards a holographic approach to spherical aberration correction in scanning transmission electron microscopy. Optics Express, 25(18):21851, 2017. Cited on page 10.

[60] G. Guzzinati, L. Clark, A. Béché, R. Juchtmans, R. Van Boxem, M. Mazilu, and J. Verbeeck. Prospects for versatile phase manipulation in the TEM: Beyond aberration correction. Ultramicroscopy, 151:85-93, 2015. Cited on page 10.

[61] V. Grillo, J. Harris, G. C. Gazzadi, R. Balboni, E. Mafakheri, M. R. Dennis, S. Frabboni, R. W. Boyd, and E. Karimi. Generation and application of bessel beams in electron microscopy. Ultramicroscopy, 166:48-60, 2016. Cited on page 10.

[62] E. Karimi, L. Marrucci, V. Grillo, and E. Santamato. Spin-to-orbital angular momentum conversion and spin-polarization filtering in electron beams. Physical Review Letters, 108(4):1-5, 2012. Cited on page 10.

[63] P. Schattschneider, V. Grillo, and D. Aubry. Spin polarisation with electron Bessel beams. Ultramicroscopy, 176:188-193, 2017. Cited on page 10.

[64] J. M. Cowley. Diffraction Physics. 3rd edition, Amsterdam - Lausanne - New York - Oxford - Shannon - Tokyo: North Holland, 1995, 481 pages. Cited on pages 10, 14,88 and 91.

[65] K. Suenaga, M. Tencé, C. Mory, C. Colliex, H. Kato, T. Okazaki, H. Shinohara, K. Hirahara, S. Bandow, and S. Iijima. Element-Selective Single Atom Imaging. Science, 290(5500):2280-2282, 2000. Cited on page 11.

[66] P. A. Midgley and R. E. Dunin-Borkowski. Electron tomography and holography in materials science. Nature Materials, 8(4):271-280, 2009. Cited on pages 11 and 40.

[67] F. Houdellier, C. Roucau, L. Clément, J. Rouvière, and M. Casanove. Quantitative analysis of HOLZ line splitting in CBED patterns of epitaxially strained layers. Ultramicroscopy, 106(10):951-959, 2006. Cited on pages 12 and 86.

[68] M. Hÿtch, F. Houdellier, F. Hüe, and E. Snoeck. Nanoscale holographic interferometry for strain measurements in electronic devices. Nature, 453(7198):1086-1089, 2008. Cited on pages 12 and 86. 
[69] M. Hÿtch, E. Snoeck, and R. Kilaas. Quantitative measurement of displacement and strain fields from HREM micrographs. Ultramicroscopy, 74(3):131-146, 1998. Cited on pages 12, 86, 98 and 105 .

[70] J.-M. Zuo, A. B. Shah, H. Kim, Y. Meng, W. Gao, and J.-L. Rouviére. Lattice and strain analysis of atomic resolution Z-contrast images based on template matching. Ultramicroscopy, 136:50-60, 2014. Cited on page 12.

[71] M. J. Hÿtch, J.-L. Putaux, and J.-M. Pénisson. Measurement of the displacement field of dislocations to $0.03 \AA$ by electron microscopy. Nature, 423(6937):270-273, 2003. Cited on page 12.

[72] A. Béché, J. L. Rouvière, L. Clément, and J. M. Hartmann. Improved precision in strain measurement using nanobeam electron diffraction. Applied Physics Letters, 95(12):123114, 2009. Cited on page 13.

[73] J. Zuo. Automated lattice parameter measurement from HOLZ lines and their use for the measurement of oxygen content in $\mathrm{YBa} 2 \mathrm{Cu} 3 \mathrm{O} 7-\delta$ from nanometer-sized region. Ultramicroscopy, 41(1-3):211-223, 1992. Cited on pages 13 and 92.

[74] C. Davisson and L. H. Germer. Diffraction of Electrons by a Crystal of Nickel. Physical Review, 30(6):705-740, 1927. Cited on page 14.

[75] S. Schäfer, W. Liang, and A. H. Zewail. Primary structural dynamics in graphite. New Journal of Physics, 13(6):063030, 2011. Cited on pages 15, 95, 99 and 104.

[76] W. Liang, G. M. Vanacore, and A. H. Zewail. Observing (non)linear lattice dynamics in graphite by ultrafast Kikuchi diffraction. Proceedings of the National Academy of Sciences, 111(15):5491-5496, 2014. Cited on pages 15 and 95.

[77] S. Vogelgesang, G. Storeck, J. G. Horstmann, T. Diekmann, M. Sivis, S. Schramm, K. Rossnagel, S. Schäfer, and C. Ropers. Phase ordering of charge density waves traced by ultrafast low-energy electron diffraction. Nature Physics, 14(2):184-190, 2018. Cited on pages 16 and 124.

[78] T. Frigge, B. Hafke, T. Witte, B. Krenzer, C. Streubühr, A. Samad Syed, V. Mikšić Trontl, I. Avigo, P. Zhou, M. Ligges, D. von der Linde, U. Bovensiepen, M. Hornvon Hoegen, S. Wippermann, A. Lücke, S. Sanna, U. Gerstmann, and W. G. Schmidt. Optically excited structural transition in atomic wires on surfaces at the quantum limit. Nature, 544(7649):207-211, 2017. Cited on pages 16, 86 and 124. 
[79] S. Volz, J. Ordonez-Miranda, A. Shchepetov, M. Prunnila, J. Ahopelto, T. Pezeril, G. Vaudel, V. Gusev, P. Ruello, E. M. Weig, M. Schubert, M. Hettich, M. Grossman, T. Dekorsy, F. Alzina, B. Graczykowski, E. Chavez-Angel, J. Sebastian Reparaz, M. R. Wagner, C. M. Sotomayor-Torres, S. Xiong, S. Neogi, and D. Donadio. Nanophononics: state of the art and perspectives. The European Physical Journal $B, 89(1): 15,2016$. Cited on pages 17, 86 and 121 .

[80] D. J. Flannigan, P. C. Samartzis, A. Yurtsever, and A. H. Zewail. Nanomechanical motions of cantilevers: direct imaging in real space and time with 4D electron microscopy. Nano Letters, 9(2):875-881, 2009. Cited on page 17.

[81] D. J. Flannigan, S. T. Park, and A. H. Zewail. Nanofriction Visualized in Space and Time by 4D Electron Microscopy. Nano Letters, 10(11):4767-4773, 2010. Cited on page 17 .

[82] B. Barwick, H. S. Park, O.-h. Kwon, J. S. Baskin, and A. H. Zewail. 4D imaging of transient structures and morphologies in ultrafast electron microscopy. Science, 322(5905):1227-1231, 2008. Cited on pages 17, 40 and 86.

[83] O.-H. Kwon, B. Barwick, H. S. Park, J. S. Baskin, and A. H. Zewail. Nanoscale Mechanical Drumming Visualized by 4D Electron Microscopy. Nano Letters, 8(11):3557-3562, 2008. Cited on pages 17, 86 and 97.

[84] D. R. Cremons, D. A. Plemmons, and D. J. Flannigan. Femtosecond electron imaging of defect-modulated phonon dynamics. Nature Communications, 7:11230, 2016. Cited on pages 17 and 86.

[85] A. J. McKenna, J. K. Eliason, and D. J. Flannigan. Spatiotemporal Evolution of Coherent Elastic Strain Waves in a Single MoS $<\mathrm{sub}>2</$ sub $>$ Flake. Nano Letters, 17(6):3952-3958, 2017. Cited on pages 17 and 86.

[86] D. R. Cremons, D. A. Plemmons, and D. J. Flannigan. Defect-mediated phonon dynamics in TaS2 and WSe2. Structural Dynamics, 4(4):044019, 2017. Cited on page 17.

[87] D. R. Cremons, D. X. Du, and D. J. Flannigan. Picosecond phase-velocity dispersion of hypersonic phonons imaged with ultrafast electron microscopy. Physical Review Materials, 1(7):073801, 2017. Cited on page 17. 
[88] A. Yurtsever and A. H. Zewail. Kikuchi ultrafast nanodiffraction in fourdimensional electron microscopy. Proceedings of the National Academy of Sciences, 108(8):3152-3156, 2011. Cited on pages 17, 18 and 86.

[89] A. Yurtsever, S. Schaefer, and A. H. Zewail. Ultrafast Kikuchi Diffraction: Nanoscale Stress-Strain Dynamics of Wave-Guiding Structures. Nano Letters, 12(7):3772-3777, 2012. Cited on pages 17 and 86.

[90] B. Barwick, D. J. Flannigan, and A. H. Zewail. Photon-induced near-field electron microscopy. Nature, 462(7275):902-906, 2009. Cited on pages 18, 19, 20, 49, 55, $56,61,62,63,96$ and 106.

[91] S. T. Park, M. Lin, and A. H. Zewail. Photon-induced near-field electron microscopy (PINEM): theoretical and experimental. New Journal of Physics, 12(12):123028, 2010. Cited on pages 19, 20, 55, 56, 61, 62, 63, 65, 66, 75, 76, 79, 80, 106 and 107.

[92] R. F. Egerton. Electron energy-loss spectroscopy in the TEM. Reports on Progress in Physics, 72(1), 2009. Cited on pages 18 and 74.

[93] F. J. García de Abajo. Optical excitations in electron microscopy. Reviews of Modern Physics, 82(1):209-275, 2010. Cited on pages 18, 55, 56 and 62.

[94] H. Boersch, J. Geiger, and W. Stickel. Interaction of 25-keV Electrons with Lattice Vibrations in LiF. Experimental Evidence for Surface Modes of Lattice Vibration. Physical Review Letters, 17(7):379-381, 1966. Cited on page 18.

[95] M. J. Lagos, A. Trügler, U. Hohenester, and P. E. Batson. Mapping vibrational surface and bulk modes in a single nanocube. Nature, 543(7646):529-532, 2017. Cited on pages 18 and 122 .

[96] A. Howie. Electrons and photons: exploiting the connection. Inst. Phys. Conf. Ser. No 161: Section 7. 1999, pp. 311-314. Cited on page 18.

[97] F. J. García de Abajo and M. Kociak. Electron energy-gain spectroscopy. New Journal of Physics, 10(7):073035, 2008. Cited on pages 18 and 55.

[98] F. J. García de Abajo, A. Asenjo-Garcia, and M. Kociak. Multiphoton Absorption and Emission by Interaction of Swift Electrons with Evanescent Light Fields. Nano Letters, 10(5):1859-1863, 2010. Cited on pages 19, 20, 56, 61, 62, 63, 65, 66, 75, 77, 80, 106 and 107. 
[99] L. Piazza, T. Lummen, E. Quiñonez, Y. Murooka, B. Reed, B. Barwick, and F. Carbone. Simultaneous observation of the quantization and the interference pattern of a plasmonic near-field. Nature Communications, 6:6407, 2015. Cited on pages 19, 20, 55, 72, 96 and 106.

[100] S. T. Park, O.-H. Kwon, and A. H. Zewail. Chirped imaging pulses in fourdimensional electron microscopy: femtosecond pulsed hole burning. New Journal of Physics, 14(5):053046, 2012. Cited on pages 20, 49, 51 and 72.

[101] M. Reiser. Theory and Design of Charged Particle Beams. 2nd edition, Wiley Series in Beam Physics and Accelerator Technology. Weinheim, Germany: WileyVCH Verlag GmbH \& Co. KGaA, 2008, 674 pages. Cited on pages 21, 22, 23, 25 and 47.

[102] J. Liouville. Note on the Theory of the Variation of Arbitrary Constants. Journal de Mathématiques Pures et Appliquées, 3:342-349, 1838. Cited on page 22.

[103] C. A. Brau. What Brightness means. The Physics and Applications of High Brightness Electron Beams. Ed. by J. Rosenzweig, G. Travish, and L. Serafini. Singapore: WORLD SCIENTIFIC, 2003, pp. 20-27. Cited on pages 23, 26 and 47.

[104] L. Mandel and E. Wolf. Optical Coherence and Quantum Optics. 1st edition, Cambridge: Cambridge University Press, 1995, 1194 pages. Cited on pages 24, 68, 78 and 80 .

[105] H. Rose. Geometrical Charged-Particle Optics. 2nd edition, vol. 142. Springer Series in Optical Sciences. Berlin, Heidelberg: Springer Berlin Heidelberg, 2012, 507 pages. Cited on pages 24, 27 and 47.

[106] F. Röder and A. Lubk. Transfer and reconstruction of the density matrix in off-axis electron holography. Ultramicroscopy, 146:103-116, 2014. Cited on page 24.

[107] A. Lubk and F. Röder. Phase-space foundations of electron holography. Physical Review A, 92(3):033844, 2015. Cited on page 24.

[108] J. C. H. Spence, W. Qian, and M. P. Silverman. Electron source brightness and degeneracy from Fresnel fringes in field emission point projection microscopy. Journal of Vacuum Science \& Technology A: Vacuum, Surfaces, and Films, 12(2):542547, 1994. Cited on page 24. 
[109] G. Pozzi. Theoretical considerations of the spatial coherence in field emission electron microscopes. Optik, 77(2):69-73, 1987. Cited on pages 26 and 49.

[110] P. Baum. On the physics of ultrashort single-electron pulses for time-resolved microscopy and diffraction. Chemical Physics, 423:55-61, 2013. Cited on pages 26 and 112.

[111] T. Van Oudheusden, E. F. De Jong, S. B. van der Geer, W. P. E. M. Op 'T Root, O. J. Luiten, B. J. Siwick, W. P. E. M. Op't Root, O. J. Luiten, and B. J. Siwick. Electron source concept for single-shot sub-100 fs electron diffraction in the 100 keV range. Journal of Applied Physics, 102(9):093501, 2007. Cited on pages 26, 48, 110 and 111.

[112] W. Lauterborn and T. Kurz. Coherent Optics. Advanced Texts in Physics. Berlin, Heidelberg: Springer Berlin Heidelberg, 2003, 364 pages. Cited on page 26.

[113] H. Kohl and H. Rose. Theory of Image Formation by Inelastically Scattered Electrons in the Electron Microscope. Advances in Imaging and Electron Physics. Vol. 65. 1985, pp. 173-227. Cited on page 27.

[114] G. Sciaini and R. J. D. Miller. Femtosecond electron diffraction: heralding the era of atomically resolved dynamics. Reports on Progress in Physics, 74(9):096101, 2011. Cited on pages 28, 29 and 124 .

[115] S. Manz, A. Casandruc, D. Zhang, Y. Zhong, R. A. Loch, A. Marx, T. Hasegawa, L. C. Liu, S. Bayesteh, H. Delsim-Hashemi, M. Hoffmann, M. Felber, M. Hachmann, F. Mayet, J. Hirscht, S. Keskin, M. Hada, S. W. Epp, K. Flöttmann, and R. J. D. Miller. Mapping atomic motions with ultrabright electrons: towards fundamental limits in space-time resolution. Faraday Discussions, 177(177):467491, 2015. Cited on pages 28, 40, 44, 51, 111 and 112.

[116] S. P. Weathersby, G. Brown, M. Centurion, T. F. Chase, R. Coffee, J. Corbett, J. P. Eichner, J. C. Frisch, A. R. Fry, M. Gühr, N. Hartmann, C. Hast, R. Hettel, R. K. Jobe, E. N. Jongewaard, J. R. Lewandowski, R. K. Li, A. M. Lindenberg, I. Makasyuk, J. E. May, D. McCormick, M. N. Nguyen, A. H. Reid, X. Shen, K. Sokolowski-Tinten, T. Vecchione, S. L. Vetter, J. Wu, J. Yang, H. A. Dürr, and X. J. Wang. Mega-electron-volt ultrafast electron diffraction at SLAC National Accelerator Laboratory. Review of Scientific Instruments, 86(7):073702, 2015. Cited on pages 28, 40, 42, 44, 51, 86, 111 and 112. 
[117] P. Zhu, Y. Zhu, Y. Hidaka, L. Wu, J. Cao, H. Berger, J. Geck, R. Kraus, S. Pjerov, Y. Shen, R. I. Tobey, J. P. Hill, and X. J. Wang. Femtosecond time-resolved MeV electron diffraction. New Journal of Physics, 17(6):063004, 2015. Cited on pages 28 and 112.

[118] H. Daoud, K. Floettmann, and R. J. Dwayne Miller. Compression of high-density $0.16 \mathrm{pC}$ electron bunches through high field gradients for ultrafast single shot electron diffraction: The Compact RF Gun. Structural Dynamics, 4(4):044016, 2017. Cited on pages 28 and 111.

[119] J. Williams, F. Zhou, T. Sun, Z. Tao, K. Chang, K. Makino, M. Berz, P. M. Duxbury, and C.-Y. Ruan. Active control of bright electron beams with RF optics for femtosecond microscopy. Structural Dynamics, 4(4):044035, 2017. Cited on pages 28, 110,111 and 115 .

[120] F. Zhou, J. Williams, and C.-Y. Ruan. Femtosecond electron spectroscopy in an electron microscope with high brightness beams. Chemical Physics Letters, 683:488-494, 2017. Cited on pages 28, 110 and 115.

[121] A. Lassise, P. H. A. Mutsaers, and O. J. Luiten. Compact, low power radio frequency cavity for femtosecond electron microscopy. Review of Scientific Instruments, 83(4):0-10, 2012. Cited on page 28.

[122] W. Verhoeven, J. van Rens, E. Kieft, P. Mutsaers, and O. Luiten. High quality ultrafast transmission electron microscopy using resonant microwave cavities. Ultramicroscopy, 188:85-89, 2018. Cited on pages 28 and 115.

[123] E. W. Ernst and H. VonFoerster. Electron Bunches of Short Time Duration. Journal of Applied Physics, 25(5):674, 1954. Cited on page 28.

[124] T. Hosokawa, H. Fujioka, and K. Ura. Generation and measurement of subpicosecond electron beam pulses. Review of Scientific Instruments, 49(5):624-628, 1978. Cited on pages 28, 40 and 115 .

[125] G. Weppelman, R. Moerland, R. van Tol, C. Heerkens, J. Hoogenboom, and P. Kruit. Laser triggered microfabricated ultrafast electron beam blanker. European Microscopy Congress 2016: Proceedings. Weinheim, Germany: Wiley-VCH Verlag GmbH \& Co. KGaA, 2016, pp. 406-407. Cited on page 28. 
[126] J. van Rens, W. Verhoeven, J. Franssen, A. Lassise, X. Stragier, E. Kieft, P. Mutsaers, and O. Luiten. Theory and particle tracking simulations of a resonant radiofrequency deflection cavity in TM 110 mode for ultrafast electron microscopy. Ultramicroscopy, 184:77-89, 2018. Cited on pages 28, 29 and 115.

[127] J. Als-Nielsen and D. McMorrow. Elements of Modern X-ray Physics. 2nd edition, Hoboken, NJ, USA: John Wiley \& Sons, Inc., 2011, 434 pages. Cited on page 29.

[128] J. J. Thomson. XL. Cathode Rays. The London, Edinburgh, and Dublin Philosophical Magazine and Journal of Science, 44(269):293-316, 1897. Cited on page 29.

[129] D. T. Pierce, R. J. Celotta, G. Wang, W. N. Unertl, A. Galejs, C. E. Kuyatt, and S. R. Mielczarek. The GaAs spin polarized electron source. Review of Scientific Instruments, 51(4):478-499, 1980. Cited on page 30.

[130] C. Kittel. Einführung in die Festkörperphysik. 14th edition, München: Oldenbourg Wissenschaftsverlag, 2006, 776 pages. Cited on pages 30 and 31.

[131] C. Todd and T. Rhodin. Work function in field emission - the (110) plane of tungsten. Surface Science, 36(1):353-369, 1973. Cited on page 31.

[132] L. Swanson, M. Gesley, and P. Davis. Crystallographic dependence of the work function and volatility of LaB6. Surface Science, 107(1):263-289, 1981. Cited on page 31 .

[133] D. H. Dowell and J. F. Schmerge. Quantum efficiency and thermal emittance of metal photocathodes. Physical Review Special Topics - Accelerators and Beams, 12(7):074201, 2009. Cited on pages 32, 35, 37, 44 and 110.

[134] W. Schottky. Über kalte und warme Elektronenentladungen. Zeitschrift für Physik, 14(1):63-106, 1923. Cited on page 32.

[135] M. K. Miller, A. Cerezo, M. G. Hetherington, and G. D. W. Smith. Atom probe field ion microscopy. Oxford: Clarendon Press, 1996, 509 pages. Cited on page 32.

[136] R. H. Fowler and L. Nordheim. Electron Emission in Intense Electric Fields. Proceedings of the Royal Society A: Mathematical, Physical and Engineering Sciences, 119(781):173-181, 1928. Cited on page 33.

[137] R. G. Forbes. Refining the application of Fowler-Nordheim theory. Ultramicroscopy, 79(1-4):11-23, 1999. Cited on page 33. 
[138] L. W. Swanson and G. A. Schwind. Review of ZrO/W Schottky cathode. Handbook of charged particle optics. Ed. by J. Orloff. Vol. 2nd Editio. CRC Press, 2008. Chap. Review of. Cited on pages 33, 34 and 45.

[139] M. S. Bronsgeest and P. Kruit. Temperature dependence of the work function of the $\mathrm{Zr} / \mathrm{O} / \mathrm{W}(100)$ Schottky electron source in typical operating conditions and its effect on beam brightness. Journal of Vacuum Science \& Technology B: Microelectronics and Nanometer Structures, 24(2):887, 2006. Cited on page 33.

[140] R. Bormann, S. Strauch, S. Schäfer, and C. Ropers. An ultrafast electron microscope gun driven by two-photon photoemission from a nanotip cathode. Journal of Applied Physics, 118(17):173105, 2015. Cited on pages 34, 42, 44, 46, 49, 87 and 113.

[141] H. Hertz. Ueber einen Einfluss des ultravioletten Lichtes auf die electrische Entladung. Annalen der Physik und Chemie, 267(8):983-1000, 1887. Cited on page 34.

[142] A. Einstein. Über einen die Erzeugung und Verwandlung des Lichtes betreffenden heuristischen Gesichtspunkt. Annalen der Physik, 322(6):132-148, 1905. Cited on pages 34 and 35 .

[143] G. Ferrini, F. Banfi, C. Giannetti, and F. Parmigiani. Non-linear electron photoemission from metals with ultrashort pulses. Nuclear Instruments and Methods in Physics Research Section A: Accelerators, Spectrometers, Detectors and Associated Equipment, 601(1-2):123-131, 2009. Cited on page 35.

[144] L. A. DuBridge. Theory of the Energy Distribution of Photoelectrons. Physical Review, 43(9):727-741, 1933. Cited on page 35.

[145] J. G. Fujimoto, J. M. Liu, E. P. Ippen, and N. Bloembergen. Femtosecond Laser Interaction with Metallic Tungsten and Nonequilibrium Electron and Lattice Temperatures. Physical Review Letters, 53(19):1837-1840, 1984. Cited on page 35.

[146] M. Aidelsburger, F. O. Kirchner, F. Krausz, and P. Baum. Single-electron pulses for ultrafast diffraction. Proceedings of the National Academy of Sciences, 107(46):19714-19719, 2010. Cited on pages 35, 44 and 112.

[147] G. Herink, D. R. Solli, M. Gulde, and C. Ropers. Field-driven photoemission from nanostructures quenches the quiver motion. Nature, 483(7388):190-193, 2012. Cited on pages 36, 38, 44, 62 and 66. 
[148] R. Bormann, M. Gulde, A. Weismann, S. V. Yalunin, and C. Ropers. Tip-enhanced strong-field photoemission. Physical Review Letters, 105(14):147601, 2010. Cited on pages 36 and 44 .

[149] Z. Tao, H. Zhang, P. M. Duxbury, M. Berz, and C. Y. Ruan. Space charge effects in ultrafast electron diffraction and imaging. Journal of Applied Physics, 111, 2012. Cited on page 37.

[150] P. Hommelhoff, Y. Sortais, A. Aghajani-Talesh, and M. A. Kasevich. Field emission tip as a nanometer source of free electron femtosecond pulses. Physical Review Letters, 96(7):077401, 2006. Cited on pages 37, 44, 63 and 113.

[151] C. Ropers, D. R. Solli, C. P. Schulz, C. Lienau, and T. Elsaesser. Localized multiphoton emission of femtosecond electron pulses from metal nanotips. Physical Review Letters, 98(4):043907, 2007. Cited on pages 37, 44, 63 and 113.

[152] B. Barwick, C. Corder, J. Strohaber, N. Chandler-Smith, C. Uiterwaal, and H. Batelaan. Laser-induced ultrafast electron emission from a field emission tip. New Journal of Physics, 9(5):142-142, 2007. Cited on pages 37, 44 and 113.

[153] L. Novotny, R. X. Bian, and X. S. Xie. Theory of Nanometric Optical Tweezers. Physical Review Letters, 79(4):645-648, 1997. Cited on page 37.

[154] A. Bouhelier, M. Beversluis, A. Hartschuh, and L. Novotny. Near-Field SecondHarmonic Generation Induced by Local Field Enhancement. Physical Review Letters, 90(1):013903, 2003. Cited on page 37.

[155] S. Thomas, M. Krüger, M. Förster, M. Schenk, and P. Hommelhoff. Probing of Optical Near-Fields by Electron Rescattering on the $1 \mathrm{~nm}$ Scale. Nano Letters, 13(10):4790-4794, 2013. Cited on pages 37 and 38.

[156] R. Bormann. Development and characterization of an electron gun for ultrafast electron microscopy. $\mathrm{PhD}$ thesis. Georg-August Universität Göttingen, 2015. Cited on page 38 .

[157] D. Ehberger, J. Hammer, M. Eisele, M. Krüger, J. Noe, A. Högele, and P. Hommelhoff. Highly coherent electron beam from a laser-triggered tungsten needle tip. Physical Review Letters, 114(22):227601, 2015. Cited on pages 38, 44, 113 and 114. 
[158] B. Cook, M. Bronsgeest, K. Hagen, and P. Kruit. Improving the energy spread and brightness of thermal-field (Schottky) emitters with PHAST - PHoto Assisted Schottky Tip. Ultramicroscopy, 109(5):403-412, 2009. Cited on pages 38, 42, 45 and 113.

[159] D.-S. Yang, O. F. Mohammed, and A. H. Zewail. Scanning ultrafast electron microscopy. Proceedings of the National Academy of Sciences, 107(34):1499314998, 2010. Cited on pages 38, 40, 42, 44 and 45.

[160] R. Fernandez-Leiro and S. H. W. Scheres. Unravelling biological macromolecules with cryo-electron microscopy. Nature, 537(7620):339-346, 2016. Cited on page 40.

[161] D. S. Su, B. Zhang, and R. Schlögl. Electron Microscopy of Solid Catalysts Transforming from a Challenge to a Toolbox. Chemical Reviews, 115(8):28182882, 2015. Cited on page 40.

[162] H. Zheng, Y. S. Meng, and Y. Zhu. Frontiers of in situ electron microscopy. MRS Bulletin, 40(01):12-18, 2015. Cited on page 40.

[163] Z.-J. Wang, G. Weinberg, Q. Zhang, T. Lunkenbein, A. Klein-Hoffmann, M. Kurnatowska, M. Plodinec, Q. Li, L. Chi, R. Schloegl, and M.-g. Willinger. Direct observation of graphene growth and associated copper substrate dynamics by in situ scanning electron microscopy. ACS Nano, 9(2):1506-1519, 2015. Cited on page 40 .

[164] J. Nelson, S. Misra, Y. Yang, A. Jackson, Y. Liu, H. Wang, H. Dai, J. C. Andrews, Y. Cui, and M. F. Toney. In operando X-ray diffraction and transmission X-ray microscopy of lithium sulfur batteries. Journal of the American Chemical Society, 134(14):6337-6343, 2012. Cited on page 40.

[165] F. Endres, N. Borisenko, S. Z. El Abedin, R. Hayes, and R. Atkin. The interface ionic liquid(s)/electrode(s): in situ STM and AFM measurements. Faraday Discuss., 154:221-233, 2012. Cited on page 40.

[166] R. J. D. Miller. Femtosecond crystallography with ultrabright electrons and X-rays: capturing chemistry in action. Science, 343(6175):1108-1116, 2014. Cited on pages 40, 42, 44 and 86. 
[167] P. Musumeci, J. T. Moody, C. M. Scoby, M. S. Gutierrez, H. A. Bender, and N. S. Wilcox. High quality single shot diffraction patterns using ultrashort megaelectron volt electron beams from a radio frequency photoinjector. Review of Scientific Instruments, 81(1):013306, 2010. Cited on pages 40 and 42.

[168] S. Lahme, C. Kealhofer, F. Krausz, and P. Baum. Femtosecond single-electron diffraction. Structural Dynamics, 1(3):034303, 2014. Cited on pages 40 and 42.

[169] M. Först, A. D. Caviglia, R. Scherwitzl, R. Mankowsky, P. Zubko, V. Khanna, H. Bromberger, S. B. Wilkins, Y.-D. Chuang, W. S. Lee, W. F. Schlotter, J. J. Turner, G. L. Dakovski, M. P. Minitti, J. Robinson, S. R. Clark, D. Jaksch, J.-M. Triscone, J. P. Hill, S. S. Dhesi, and A. Cavalleri. Spatially resolved ultrafast magnetic dynamics initiated at a complex oxide heterointerface. Nature Materials, 14(9):883-888, 2015. Cited on page 40.

[170] T. Huber, S. O. Mariager, A. Ferrer, H. Schäfer, J. A. Johnson, S. Grübel, A. Lübcke, L. Huber, T. Kubacka, C. Dornes, C. Laulhe, S. Ravy, G. Ingold, P. Beaud, J. Demsar, and S. L. Johnson. Coherent structural dynamics of a prototypical charge-density-wave-to-metal transition. Physical Review Letters, 113(2):026401, 2014. Cited on page 40.

[171] M. C. Langner, S. Zhou, G. Coslovich, Y.-D. Chuang, Y. Zhu, J. S. Robinson, W. F. Schlotter, J. J. Turner, M. P. Minitti, R. G. Moore, W. S. Lee, D. H. Lu, D. Doering, P. Denes, Y. Tomioka, Y. Tokura, R. A. Kaindl, and R. W. Schoenlein. Ultrafast $\mathrm{X}$-ray and optical signatures of phase competition and separation underlying the photoinduced metallic phase in Pr1-xCaxMnO3. Physical Review $B, 92(15): 155148,2015$. Cited on page 40.

[172] W. Liang, S. Schäfer, and A. H. Zewail. Ultrafast electron crystallography of heterogeneous structures: Gold-graphene bilayer and ligand-encapsulated nanogold on graphene. Chemical Physics Letters, 542:8-12, 2012. Cited on page 40.

[173] S. Wall, B. Krenzer, S. Wippermann, S. Sanna, F. Klasing, A. Hanisch-Blicharski, M. Kammler, W. G. Schmidt, and M. Horn-von Hoegen. Atomistic picture of charge density wave formation at surfaces. Physical Review Letters, 109(18):186101, 2012. Cited on page 40. 
[174] M. Gulde, S. Schweda, G. Storeck, M. Maiti, H. K. Yu, A. M. Wodtke, S. Schafer, and C. Ropers. Ultrafast low-energy electron diffraction in transmission resolves polymer/graphene superstructure dynamics. Science, 345(6193):200-204, 2014. Cited on pages 40, 42, 44, 63, 86, 113 and 124.

[175] T. Frigge, B. Hafke, V. Tinnemann, B. Krenzer, and M. Horn-von Hoegen. Nanoscale heat transport from Ge hut, dome, and relaxed clusters on $\mathrm{Si}(001)$ measured by ultrafast electron diffraction. Applied Physics Letters, 106(5):053108, 2015. Cited on page 40.

[176] J. N. Clark, L. Beitra, G. Xiong, A. Higginbotham, D. M. Fritz, H. T. Lemke, D. Zhu, M. Chollet, G. J. Williams, M. Messerschmidt, B. Abbey, R. J. Harder, A. M. Korsunsky, J. S. Wark, and I. K. Robinson. Ultrafast three-dimensional imaging of lattice dynamics in individual gold nanocrystals. Science, 341(6141):56-59, 2013. Cited on pages 40, 86, 98, 105 and 123.

[177] P. Beaud, A. Caviezel, S. O. Mariager, L. Rettig, G. Ingold, C. Dornes, S.-W. Huang, J. A. Johnson, M. Radovic, T. Huber, T. Kubacka, A. Ferrer, H. T. Lemke, M. Chollet, D. Zhu, J. M. Glownia, M. Sikorski, A. Robert, H. Wadati, M. Nakamura, M. Kawasaki, Y. Tokura, S. L. Johnson, and U. Staub. A time-dependent order parameter for ultrafast photoinduced phase transitions. Nature Materials, 13(10):923-927, 2014. Cited on pages 40 and 86.

[178] Y. Terada, S. Yoshida, O. Takeuchi, and H. Shigekawa. Real-space imaging of transient carrier dynamics by nanoscale pump-probe microscopy. Nature Photonics, 4(12):869-874, 2010. Cited on page 40.

[179] T. L. Cocker, V. Jelic, M. Gupta, S. J. Molesky, J. A. J. Burgess, G. D. L. Reyes, L. V. Titova, Y. Y. Tsui, M. R. Freeman, and F. A. Hegmann. An ultrafast terahertz scanning tunnelling microscope. Nature Photonics, 7(8):620-625, 2013. Cited on page 40 .

[180] M. A. Huber, M. Plankl, M. Eisele, R. E. Marvel, F. Sandner, T. Korn, C. Schüller, R. F. Haglund, R. Huber, and T. L. Cocker. Ultrafast mid-infrared nanoscopy of strained vanadium dioxide nanobeams. Nano Letters, 16(2):1421-1427, 2016. Cited on pages 40 and 86 . 
[181] M. Wagner, A. S. McLeod, S. J. Maddox, Z. Fei, M. Liu, R. D. Averitt, M. M. Fogler, S. R. Bank, F. Keilmann, and D. N. Basov. Ultrafast dynamics of surface plasmons in InAs by time-resolved infrared nanospectroscopy. Nano Letters, 14(8):4529-4534, 2014. Cited on page 40.

[182] E. Quinonez, J. Handali, and B. Barwick. Femtosecond photoelectron point projection microscope. Review of Scientific Instruments, 84(10):103710, 2013. Cited on pages 40, 44, 113 and 124.

[183] M. Müller, V. Kravtsov, A. Paarmann, M. B. Raschke, and R. Ernstorfer. Nanofocused plasmon-driven sub-10 fs electron point source. ACS Photonics, 3(4):611619, 2016. Cited on pages 40, 44, 113 and 124.

[184] J. Vogelsang, J. Robin, B. J. Nagy, P. Dombi, D. Rosenkranz, M. Schiek, P. Groß, and C. Lienau. Ultrafast electron emission from a sharp metal nanotaper driven by adiabatic nanofocusing of surface plasmons. Nano Letters, 15(7):4685-4691, 2015. Cited on pages 40, 44, 113 and 124.

[185] K. Ura, H. Fujioka, and T. Hosokawa. Picosecond Pulse Stroboscopic Scanning Electron Microscope. Journal of Electron Microscopy, 27(4):247-252, 1978. Cited on page 40 .

[186] M. Merano, S. Sonderegger, A. Crottini, S. Collin, P. Renucci, E. Pelucchi, A. Malko, M. H. Baier, E. Kapon, B. Deveaud, and J.-D. Ganière. Probing carrier dynamics in nanostructures by picosecond cathodoluminescence. Nature, 438(7067):479-82, 2005. Cited on page 40.

[187] J. Sun, V. A. Melnikov, J. I. Khan, and O. F. Mohammed. Real-Space Imaging of Carrier Dynamics of Materials Surfaces by Second-Generation Four-Dimensional Scanning Ultrafast Electron Microscopy. The Journal of Physical Chemistry Letters, 6(19):3884-3890, 2015. Cited on pages 40 and 44.

[188] L. Piazza, D. Masiel, T. LaGrange, B. Reed, B. Barwick, and F. Carbone. Design and implementation of a fs-resolved transmission electron microscope based on thermionic gun technology. Chemical Physics, 423:79-84, 2013. Cited on pages 41 and 86.

[189] E. Kieft, K. B. Schliep, P. K. Suri, and D. J. Flannigan. Communication: effects of thermionic-gun parameters on operating modes in ultrafast electron microscopy. Structural Dynamics, 2(5):051101, 2015. Cited on pages 41 and 86. 
[190] G. Cao, S. Sun, Z. Li, H. Tian, H. Yang, and J. Li. Clocking the anisotropic lattice dynamics of multi-walled carbon nanotubes by four-dimensional ultrafast transmission electron microscopy. Scientific Reports, 5:8404, 2015. Cited on pages 41 and 86.

[191] K. Bücker, M. Picher, O. Crégut, T. LaGrange, B. Reed, S. Park, D. Masiel, and F. Banhart. Electron beam dynamics in an ultrafast transmission electron microscope with Wehnelt electrode. Ultramicroscopy, 171:8-18, 2016. Cited on pages 41 and 86.

[192] M. Kuwahara, Y. Nambo, K. Aoki, K. Sameshima, X. Jin, T. Ujihara, H. Asano, K. Saitoh, Y. Takeda, and N. Tanaka. The Boersch effect in a picosecond pulsed electron beam emitted from a semiconductor photocathode. Applied Physics Letters, 109(1):013108, 2016. Cited on pages 41 and 86.

[193] F. Houdellier, G. M. Caruso, P. Abeilhou, and A. Arbouet. Design and realization of an ultrafast cold field emission source operating under high voltage. The 16th European Microscopy Congress. Lyon, France, 2016. Cited on page 41.

[194] E. J. Kirkland. Advanced Computing in Electron Microscopy. Boston, MA: Springer US, 2010. Cited on page 41.

[195] S. Wall, D. Wegkamp, L. Foglia, K. Appavoo, J. Nag, R. F. Haglund, J. Stähler, and $\mathrm{M}$. Wolf. Ultrafast changes in lattice symmetry probed by coherent phonons. Nature Communications, 3:721, 2012. Cited on page 42.

[196] V. R. Morrison, R. P. Chatelain, K. L. Tiwari, A. Hendaoui, A. Bruhacs, M. Chaker, and B. J. Siwick. A photoinduced metal-like phase of monoclinic VO2 revealed by ultrafast electron diffraction. Science, 346(6208):445-448, 2014. Cited on pages 42 and 86.

[197] K. Haupt, M. Eichberger, N. Erasmus, A. Rohwer, J. Demsar, K. Rossnagel, and H. Schwoerer. Ultrafast Metamorphosis of a Complex Charge-Density Wave. Physical Review Letters, 116(1):016402, 2016. Cited on page 42.

[198] L. Kasmi, D. Kreier, M. Bradler, E. Riedle, and P. Baum. Femtosecond singleelectron pulses generated by two-photon photoemission close to the work function. New Journal of Physics, 17(3):33008, 2015. Cited on pages 44, 110 and 112. 
[199] A. Janzen, B. Krenzer, O. Heinz, P. Zhou, D. Thien, A. Hanisch, F.-J. Meyer zu Heringdorf, D. von der Linde, and M. Horn von Hoegen. A pulsed electron gun for ultrafast electron diffraction at surfaces. Review of Scientific Instruments, 78(1):013906, 2007. Cited on pages 44 and 110.

[200] M. Kuwahara, S. Kusunoki, Y. Nambo, K. Saitoh, X. Jin, T. Ujihara, H. Asano, Y. Takeda, and N. Tanaka. Coherence of a spin-polarized electron beam emitted from a semiconductor photocathode in a transmission electron microscope. Applied Physics Letters, 105(19):193101, 2014. Cited on pages 44 and 110.

[201] C. Gerbig, A. Senftleben, S. Morgenstern, C. Sarpe, and T. Baumert. Spatiotemporal resolution studies on a highly compact ultrafast electron diffractometer. New Journal of Physics, 17(4):43050, 2015. Cited on pages 44, 110, 112 and 124.

[202] M. Merano, S. Collin, P. Renucci, M. Gatri, S. Sonderegger, A. Crottini, J. D. Ganière, and B. Deveaud. High brightness picosecond electron gun. Review of Scientific Instruments, 76(8):085108, 2005. Cited on pages 44 and 110.

[203] B. J. Claessens, S. B. van der Geer, G. Taban, E. J. D. Vredenbregt, and O. J. Luiten. Ultracold electron source. Physical Review Letters, 95(16):164801, 2005. Cited on pages 44 and 110 .

[204] W. J. Engelen, M. A. van der Heijden, D. J. Bakker, E. J. D. Vredenbregt, and O. J. Luiten. High-coherence electron bunches produced by femtosecond photoionization. Nature Communications, 4:1693, 2013. Cited on page 44.

[205] A. J. McCulloch, D. V. Sheludko, S. D. Saliba, S. C. Bell, M. Junker, K. A. Nugent, and R. E. Scholten. Arbitrarily shaped high-coherence electron bunches from cold atoms. Nature Physics, 7(10):785-788, 2011. Cited on page 44.

[206] B. Schröder, M. Sivis, R. Bormann, S. Schäfer, and C. Ropers. An ultrafast nanotip electron gun triggered by grating-coupled surface plasmons. Applied Physics Letters, 107(23):231105, 2015. Cited on pages 44, 46 and 113.

[207] H. Yanagisawa, C. Hafner, P. Doná, M. Klöckner, D. Leuenberger, T. Greber, J. Osterwalder, and M. Hengsberger. Laser-induced field emission from a tungsten tip: optical control of emission sites and the emission process. Physical Review B, 81(11):115429, 2010. Cited on page 44. 
[208] H. Yanagisawa. Site-selective field emission source by femtosecond laser pulses and its emission mechanism. Annalen der Physik, 525(1-2):126-134, 2013. Cited on page 44 .

[209] D. J. Park, B. Piglosiewicz, S. Schmidt, H. Kollmann, M. Mascheck, and C. Lienau. Strong field acceleration and steering of ultrafast electron pulses from a sharp metallic nanotip. Physical Review Letters, 109(24):244803, 2012. Cited on page 44.

[210] M. Krüger, M. Schenk, M. Förster, and P. Hommelhoff. Attosecond physics in photoemission from a metal nanotip. Journal of Physics B: Atomic, Molecular and Optical Physics, 45:074006, 2012. Cited on page 44.

[211] M. Schenk, M. Krüger, and P. Hommelhoff. Strong-field above-threshold photoemission from sharp metal tips. Physical Review Letters, 105(25):257601, 2010. Cited on page 44.

[212] A. Paarmann, M. Gulde, M. Müller, S. Schäfer, S. Schweda, M. Maiti, C. Xu, T. Hohage, F. Schenk, C. Ropers, and R. Ernstorfer. Coherent femtosecond low-energy single-electron pulses for time-resolved diffraction and imaging: a numerical study. Journal of Applied Physics, 112(11):113109, 2012. Cited on pages 44 and 113.

[213] J. Hoffrogge, J. Paul Stein, M. Krüger, M. Förster, J. Hammer, D. Ehberger, P. Baum, and P. Hommelhoff. Tip-based source of femtosecond electron pulses at 30 keV. Journal of Applied Physics, 115(9), 2014. Cited on page 44.

[214] L. Waldecker, R. Bertoni, and R. Ernstorfer. Compact femtosecond electron diffractometer with $100 \mathrm{keV}$ electron bunches approaching the single-electron pulse duration limit. Journal of Applied Physics, 117(4):044903, 2015. Cited on pages 44, 51, 110, 112 and 124.

[215] A. R. Bainbridge, C. W. Barlow Myers, and W. A. Bryan. Femtosecond fewto single-electron point-projection microscopy for nanoscale dynamic imaging. Structural Dynamics, 3(2):023612, 2016. Cited on pages 44 and 113.

[216] J. Spence, T. Vecchione, and U. Weierstall. A coherent photofield electron source for fast diffractive and point-projection imaging. Philosophical Magazine, 90(3536):4691-4702, 2010. Cited on page 44. 
[217] M. J. Fransen, M. H. F. Overwijk, and P. Kruit. Brightness measurements of a $\mathrm{ZrO} / \mathrm{W}$ Schottky electron emitter in a transmission electron microscope. Applied Surface Science, 146(1):357-362, 1999. Cited on pages 45 and 49.

[218] A. H. V. van Veen, C. W. Hagen, J. E. Barth, and P. Kruit. Reduced brightness of the ZrO/W Schottky electron emitter. Journal of Vacuum Science \& Technology B: Microelectronics and Nanometer Structures, 19(6):2038, 2001. Cited on pages 45 and 49.

[219] B. Cook, T. Verduin, C. W. Hagen, and P. Kruit. Brightness limitations of cold field emitters caused by Coulomb interactions. Journal of Vacuum Science \& Technology B: Microelectronics and Nanometer Structures, 28(6):C6C74, 2010. Cited on page 47.

[220] W. Graves, L. DiMauro, R. Heese, E. Johnson, J. Rose, J. Rudati, T. Shaftan, and B. Sheehy. Measurement of thermal emittance for a copper photocathode. Proceedings of the PACS2001 Particle Accelerator Conference (Cat. No.01CH37268). Vol. 3. IEEE, 2001, pp. 2227-2229. Cited on page 47.

[221] B. L. Rickman, J. A. Berger, A. W. Nicholls, and W. A. Schroeder. Intrinsic electron beam emittance from metal photocathodes: the effect of the electron effective mass. Physical Review Letters, 111(23):237401, 2013. Cited on page 47.

[222] S. T. Park and A. H. Zewail. Relativistic effects in photon-induced near field electron microscopy. The Journal of Physical Chemistry A, 116(46):11128-11133, 2012. Cited on pages 49 and 75.

[223] F. O. Kirchner, A. Gliserin, F. Krausz, and P. Baum. Laser streaking of free electrons at $25 \mathrm{keV}$. Nature Photonics, 8(1):52-57, 2013. Cited on pages 49, 55, 61 and 63.

[224] B. J. Siwick, A. A. Green, C. T. Hebeisen, and R. J. D. Miller. Characterization of ultrashort electron pulses by electron-laser pulse cross correlation. Optics Letters, 30(9):1057, 2005. Cited on page 49.

[225] D. A. Plemmons, S. T. Park, A. H. Zewail, and D. J. Flannigan. Characterization of fast photoelectron packets in weak and strong laser fields in ultrafast electron microscopy. Ultramicroscopy, 146:97-102, 2014. Cited on pages 49, 51, 55 and 72. 
[226] C. T. Hebeisen, G. Sciaini, M. Harb, R. Ernstorfer, T. Dartigalongue, S. G. Kruglik, and R. J. D. Miller. Grating enhanced ponderomotive scattering for visualization and full characterization of femtosecond electron pulses. Optics Express, 16(5):3334, 2008. Cited on page 49.

[227] G. Berden, S. P. Jamison, A. M. MacLeod, W. A. Gillespie, B. Redlich, and A. F. G. van der Meer. Electro-optic technique with improved time resolution for real-time, nondestructive, single-shot measurements of femtosecond electron bunch profiles. Physical Review Letters, 93(11):114802, 2004. Cited on page 49.

[228] T. van Oudheusden, P. L. E. M. Pasmans, S. B. van der Geer, M. J. de Loos, M. J. van der Wiel, and O. J. Luiten. Compression of subrelativistic space-chargedominated electron bunches for single-shot femtosecond electron diffraction. Physical Review Letters, 105(26):264801, 2010. Cited on pages 51 and 111.

[229] C. Kealhofer, W. Schneider, D. Ehberger, A. Ryabov, F. Krausz, and P. Baum. All-optical control and metrology of electron pulses. Science, 352(6284):429-433, 2016. Cited on pages 51, 115 and 116.

[230] W. Verhoeven, J. F. M. van Rens, M. A. W. van Ninhuijs, W. F. Toonen, E. R. Kieft, P. H. A. Mutsaers, and O. J. Luiten. Time-of-flight electron energy loss spectroscopy using TM110 deflection cavities. Structural Dynamics, 3(5):054303, 2016. Cited on pages 51 and 115.

[231] L. Wimmer, G. Herink, D. R. Solli, S. V. Yalunin, K. E. Echternkamp, and C. Ropers. Terahertz control of nanotip photoemission. Nature Physics, 10(6):432436, 2014. Cited on pages 51, 62, 66 and 116.

[232] R. P. Chatelain, V. R. Morrison, C. Godbout, and B. J. Siwick. Ultrafast electron diffraction with radio-frequency compressed electron pulses. Applied Physics Letters, 101(8):081901, 2012. Cited on pages 51 and 111.

[233] R. K. Li and P. Musumeci. Single-shot MeV transmission electron microscopy with picosecond temporal resolution. Physical Review Applied, 2(2):024003, 2014. Cited on pages 51 and 111.

[234] B. Cook and P. Kruit. Coulomb interactions in sharp tip pulsed photo field emitters. Applied Physics Letters, 109(15):151901, 2016. Cited on pages 51 and 113. 
[235] T. Eggebrecht, M. Möller, J. G. Gatzmann, N. Rubiano da Silva, A. Feist, U. Martens, H. Ulrichs, M. Münzenberg, C. Ropers, and S. Schäfer. Light-Induced Metastable Magnetic Texture Uncovered by in situ Lorentz Microscopy. Physical Review Letters, 118(9):097203, 2017. Cited on page 54.

[236] K. E. Echternkamp, A. Feist, S. Schäfer, and C. Ropers. Ramsey-type phase control of free-electron beams. Nature Physics, 12(11):1000-1004, 2016. Cited on pages $55,57,117,118$ and 120.

[237] E. Jones, M. Becker, J. Luiten, and H. Batelaan. Laser control of electron matter waves. Laser \& Photonics Reviews, 229(2):214-229, 2016. Cited on pages 55 and 120.

[238] B. Schröder, T. Weber, S. V. Yalunin, T. Kiel, C. Matyssek, M. Sivis, S. Schäfer, F. von Cube, S. Irsen, K. Busch, C. Ropers, and S. Linden. Real-space imaging of nanotip plasmons using electron energy loss spectroscopy. Physical Review B, 92(8):085411, 2015. Cited on page 55.

[239] S. V. Yalunin, B. Schröder, and C. Ropers. Theory of electron energy loss near plasmonic wires, nanorods, and cones. Physical Review B, 93(11):115408, 2016. Cited on page 55 .

[240] E. J. R. Vesseur, R. de Waele, M. Kuttge, and A. Polman. Direct observation of plasmonic modes in Au nanowires using high-resolution cathodoluminescence spectroscopy. Nano Letters, 7(9):2843-2846, 2007. Cited on page 55.

[241] J. Nelayah, M. Kociak, O. Stéphan, F. J. García de Abajo, M. Tencé, L. Henrard, D. Taverna, I. Pastoriza-Santos, L. M. Liz-Marzán, and C. Colliex. Mapping surface plasmons on a single metallic nanoparticle. Nature Physics, 3(5):348-353, 2007. Cited on page 55 .

[242] M. Bosman, V. J. Keast, M. Watanabe, A. I. Maaroof, and M. B. Cortie. Mapping surface plasmons at the nanometre scale with an electron beam. Nanotechnology, 18(16):165505, 2007. Cited on page 55.

[243] S. A. Maier. Plasmonics: fundamentals and applications. Boston, MA: Springer US, 2007. Cited on page 56.

[244] F. Krausz and M. Ivanov. Attosecond physics. Reviews of Modern Physics, 81(1):163-234, 2009. Cited on pages 59, 61, 68 and 123. 
[245] D. Bouwmeester, A. Ekert, and A. Zeilinger, eds. The Physics of Quantum Information. Berlin, Heidelberg: Springer Berlin Heidelberg, 2000, 315 pages. Cited on pages 61 and 68 .

[246] J. Itatani, J. Levesque, D. Zeidler, H. Niikura, H. Pépin, J. C. Kieffer, P. B. Corkum, and D. M. Villeneuve. Tomographic imaging of molecular orbitals. $\mathrm{Na}$ ture, 432(7019):867-871, 2004. Cited on page 61.

[247] E. A. Peralta, K. Soong, R. J. England, E. R. Colby, Z. Wu, B. Montazeri, C. McGuinness, J. McNeur, K. J. Leedle, D. Walz, E. B. Sozer, B. Cowan, B. Schwartz, G. Travish, and R. L. Byer. Demonstration of electron acceleration in a laser-driven dielectric microstructure. Nature, 503(7474):91-94, 2013. Cited on pages 61 and 62.

[248] J. Breuer and P. Hommelhoff. Laser-Based Acceleration of Nonrelativistic Electrons at a Dielectric Structure. Physical Review Letters, 111(13):134803, 2013. Cited on pages 61 and 62 .

[249] B. Shore and J. Eberly. Analytic approximations in multi-level excitation theory. Optics Communications, 24(1):83-88, 1978. Cited on pages 61, 65 and 76.

[250] D. Bouwmeester, I. Marzoli, G. Karman, W. Schleich, and J. Woerdman. Optical Galton board. Physical Review A, 61(1):013410, 1999. Cited on pages 61 and 65.

[251] E. Hemsing, G. Stupakov, D. Xiang, and A. Zholents. Beam by design: Laser manipulation of electrons in modern accelerators. Reviews of Modern Physics, 86(3):897-941, 2014. Cited on pages 62 and 66.

[252] M. Krüger, M. Schenk, and P. Hommelhoff. Attosecond control of electrons emitted from a nanoscale metal tip. Nature, 475(7354):78-81, 2011. Cited on page 62.

[253] A. Schiffrin, T. Paasch-Colberg, N. Karpowicz, V. Apalkov, D. Gerster, S. Mühlbrandt, M. Korbman, J. Reichert, M. Schultze, S. Holzner, J. V. Barth, R. Kienberger, R. Ernstorfer, V. S. Yakovlev, M. I. Stockman, and F. Krausz. Optical-field-induced current in dielectrics. Nature, 493(7430):70-74, 2012. Cited on page 62 .

[254] B. Piglosiewicz, S. Schmidt, D. J. Park, J. Vogelsang, P. Groß, C. Manzoni, P. Farinello, G. Cerullo, and C. Lienau. Carrier-envelope phase effects on the strongfield photoemission of electrons from metallic nanostructures. Nature Photonics, 8(1):37-42, 2014. Cited on page 62. 
[255] M. I. Stockman, M. F. Kling, U. Kleineberg, and F. Krausz. Attosecond nanoplasmonicfield microscope. Nature Photonics, 1(9):539-544, 2007. Cited on pages 62 and 66.

[256] P. L. Kapitza and P. A. M. Dirac. The reflection of electrons from standing light waves. Mathematical Proceedings of the Cambridge Philosophical Society, 29(02):297, 1933. Cited on page 62.

[257] D. L. Freimund, K. Aflatooni, and H. Batelaan. Observation of the Kapitza-Dirac effect. Nature, 413(6852):142-143, 2001. Cited on pages 62 and 120.

[258] S. J. Smith and E. M. Purcell. Visible Light from Localized Surface Charges Moving across a Grating. Physical Review, 92(4):1069-1069, 1953. Cited on page 62.

[259] K. Mizuno, J. Pae, T. Nozokido, and K. Furuya. Experimental evidence of the inverse Smith-Purcell effect. Nature, 328(6125):45-47, 1987. Cited on page 62.

[260] D. J. Flannigan, B. Barwick, and A. H. Zewail. Biological imaging with 4D ultrafast electron microscopy. Proceedings of the National Academy of Sciences, 107(22):9933-9937, 2010. Cited on pages 62 and 63.

[261] A. Yurtsever, R. M. van der Veen, and A. H. Zewail. Subparticle Ultrafast Spectrum Imaging in 4D Electron Microscopy. Science, 335(6064):59-64, 2012. Cited on pages $62,63,96$ and 106.

[262] M. G. Moharam and L. Young. Criterion for Bragg and Raman-Nath diffraction regimes. Applied Optics, 17(11):1757, 1978. Cited on page 66.

[263] P. Baum and A. H. Zewail. Attosecond electron pulses for 4D diffraction and microscopy. Proceedings of the National Academy of Sciences, 104(47):1840918414, 2007. Cited on pages 66 and 119.

[264] W. B. Case, M. Tomandl, S. Deachapunya, and M. Arndt. Realization of optical carpets in the Talbot and Talbot-Lau configurations. Optics Express, 17(23):20966, 2009. Cited on page 68.

[265] S. E. Harris and A. V. Sokolov. Subfemtosecond Pulse Generation by Molecular Modulation. Physical Review Letters, 81(14):2894-2897, 1998. Cited on page 68. 
[266] S. Schmidt, B. Piglosiewicz, D. Sadiq, J. Shirdel, J. S. Lee, P. Vasa, N. Park, D.-S. Kim, and C. Lienau. Adiabatic Nanofocusing on Ultrasmooth Single-Crystalline Gold Tapers Creates a 10-nm-Sized Light Source with Few-Cycle Time Resolution. ACS Nano, 6(7):6040-6048, 2012. Cited on page 75.

[267] J. P. Ibe, P. P. Bey, S. L. Brandow, R. A. Brizzolara, N. A. Burnham, D. P. DiLella, K. P. Lee, C. R. K. Marrian, and R. J. Colton. On the electrochemical etching of tips for scanning tunneling microscopy. Journal of Vacuum Science \& Technology A: Vacuum, Surfaces, and Films, 8(4):3570-3575, 1990. Cited on page 75.

[268] A. Asenjo-Garcia and F. J. García de Abajo. Plasmon electron energy-gain spectroscopy. New Journal of Physics, 15(10):103021, 2013. Cited on page 75.

[269] J. Schäfer, S.-C. Lee, and A. Kienle. Calculation of the near fields for the scattering of electromagnetic waves by multiple infinite cylinders at perpendicular incidence. Journal of Quantitative Spectroscopy and Radiative Transfer, 113(16):2113-2123, 2012. Cited on page 80.

[270] M. Maldovan. Sound and heat revolutions in phononics. Nature, 503(7475):209217, 2013. Cited on page 86.

[271] R. Venkatasubramanian, E. Siivola, T. Colpitts, and B. O'Quinn. Thin-film thermoelectric devices with high room-temperature figures of merit. Nature, 413(6856):597-602, 2001. Cited on page 86.

[272] K. M. Hoogeboom-Pot, J. N. Hernandez-Charpak, X. Gu, T. D. Frazer, E. H. Anderson, W. Chao, R. W. Falcone, R. Yang, M. M. Murnane, H. C. Kapteyn, and D. Nardi. A new regime of nanoscale thermal transport: Collective diffusion increases dissipation efficiency. Proceedings of the National Academy of Sciences, 112(16):4846-4851, 2015. Cited on page 86.

[273] M. Aspelmeyer, T. J. Kippenberg, and F. Marquardt. Cavity optomechanics. Reviews of Modern Physics, 86(4):1391-1452, 2014. Cited on pages 86 and 122.

[274] E. Verhagen, S. Deléglise, S. Weis, A. Schliesser, and T. J. Kippenberg. Quantumcoherent coupling of a mechanical oscillator to an optical cavity mode. Nature, 482(7383):63-67, 2012. Cited on pages 86 and 122. 
[275] N. D. Lanzillotti-Kimura, A. Fainstein, B. Perrin, B. Jusserand, A. Soukiassian, X. X. Xi, and D. G. Schlom. Enhancement and Inhibition of Coherent Phonon Emission of a Ni Film in a BaTiO3/SrTiO3 Cavity. Physical Review Letters, 104(18):187402, 2010. Cited on page 86.

[276] K. Shinokita, K. Reimann, M. Woerner, T. Elsaesser, R. Hey, and C. Flytzanis. Strong Amplification of Coherent Acoustic Phonons by Intraminiband Currents in a Semiconductor Superlattice. Physical Review Letters, 116(7):1-5, 2016. Cited on page 86.

[277] C. W. Chang, D. Okawa, A. Majumdar, and A. Zettl. Solid-State Thermal Rectifier. Science, 314(5802):1121-1124, 2006. Cited on page 86.

[278] E. Rousseau, A. Siria, G. Jourdan, S. Volz, F. Comin, J. Chevrier, and J.-J. Greffet. Radiative heat transfer at the nanoscale. Nature Photonics, 3(9):514-517, 2009. Cited on page 86.

[279] S. Shen, A. Narayanaswamy, and G. Chen. Surface Phonon Polaritons Mediated Energy Transfer between Nanoscale Gaps. Nano Letters, 9(8):2909-2913, 2009. Cited on page 86.

[280] K. Kloppstech, N. Könne, S.-A. Biehs, A. W. Rodriguez, L. Worbes, D. Hellmann, and A. Kittel. Giant heat transfer in the crossover regime between conduction and radiation. Nature Communications, 8:1-5, 2017. Cited on page 86.

[281] M. Eichenfield, J. Chan, R. M. Camacho, K. J. Vahala, and O. Painter. Optomechanical crystals. Nature, 462(7269):78-82, 2009. Cited on pages 86 and 122.

[282] I. S. Grudinin, H. Lee, O. Painter, and K. J. Vahala. Phonon Laser Action in a Tunable Two-Level System. Physical Review Letters, 104(8):083901, 2010. Cited on page 86.

[283] I. Mahboob, K. Nishiguchi, A. Fujiwara, and H. Yamaguchi. Phonon Lasing in an Electromechanical Resonator. Physical Review Letters, 110(12):127202, 2013. Cited on page 86.

[284] C. Thomsen, H. T. Grahn, H. J. Maris, and J. Tauc. Surface generation and detection of phonons by picosecond light pulses. Physical Review B, 34(6):4129-4138, 1986. Cited on pages 86 and 95 . 
[285] K. A. Nelson. Stimulated Brillouin scattering and optical excitation of coherent shear waves. Journal of Applied Physics, 53(9):6060-6063, 1982. Cited on pages 86 and 94.

[286] J. Cuffe, E. Chávez, A. Shchepetov, P.-O. Chapuis, E. H. El Boudouti, F. Alzina, T. Kehoe, J. Gomis-Bresco, D. Dudek, Y. Pennec, B. Djafari-Rouhani, M. Prunnila, J. Ahopelto, and C. M. Sotomayor Torres. Phonons in Slow Motion: Dispersion Relations in Ultrathin Si Membranes. Nano Letters, 12(7):3569-3573, 2012. Cited on page 86 .

[287] A. Bojahr, M. Herzog, S. Mitzscherling, L. Maerten, D. Schick, J. Goldshteyn, W. Leitenberger, R. Shayduk, P. Gaal, and M. Bargheer. Brillouin scattering of visible and hard X-ray photons from optically synthesized phonon wavepackets. Optics Express, 21(18):21188, 2013. Cited on page 86.

[288] A. Bojahr, M. Gohlke, W. Leitenberger, J. Pudell, M. Reinhardt, A. von Reppert, M. Roessle, M. Sander, P. Gaal, and M. Bargheer. Second Harmonic Generation of Nanoscale Phonon Wave Packets. Physical Review Letters, 115(19):195502, 2015. Cited on page 86 .

[289] A. Kirilyuk, A. V. Kimel, and T. Rasing. Ultrafast optical manipulation of magnetic order. Reviews of Modern Physics, 82(3):2731-2784, 2010. Cited on page 86.

[290] V. V. Temnov. Ultrafast acousto-magneto-plasmonics. Nature Photonics, 6(11):728736, 2012. Cited on page 86.

[291] R. Bertoni, M. Lorenc, H. Cailleau, A. Tissot, J. Laisney, M.-L. Boillot, L. Stoleriu, A. Stancu, C. Enachescu, and E. Collet. Elastically driven cooperative response of a molecular material impacted by a laser pulse. Nature Materials, 15(6):606-610, 2016. Cited on page 86.

[292] M. Mitrano, A. Cantaluppi, D. Nicoletti, S. Kaiser, A. Perucchi, S. Lupi, P. Di Pietro, D. Pontiroli, M. Riccò, S. R. Clark, D. Jaksch, and A. Cavalleri. Possible light-induced superconductivity in K3C60 at high temperature. Nature, 530(7591):461-464, 2016. Cited on page 86.

[293] L. Waldecker, T. a. Miller, M. Rudé, R. Bertoni, J. Osmond, V. Pruneri, R. E. Simpson, R. Ernstorfer, and S. Wall. Time-domain separation of optical properties from structural transitions in resonantly bonded materials. Nature Materials, 14(10):991-995, 2015. Cited on page 86. 
[294] F. Carbone, P. Baum, P. Rudolf, and A. H. Zewail. Structural Preablation Dynamics of Graphite Observed by Ultrafast Electron Crystallography. Physical Review Letters, 100(3):035501, 2008. Cited on pages 86, 95 and 104.

[295] T. Ishikawa, S. A. Hayes, S. Keskin, G. Corthey, M. Hada, K. Pichugin, A. Marx, J. Hirscht, K. Shionuma, K. Onda, Y. Okimoto, S.-y. Koshihara, T. Yamamoto, H. Cui, M. Nomura, Y. Oshima, M. Abdel-Jawad, R. Kato, and R. J. D. Miller. Direct observation of collective modes coupled to molecular orbital-driven charge transfer. Science, 350(6267):1501-1505, 2015. Cited on page 86.

[296] R. K. Raman, Y. Murooka, C.-Y. Ruan, T. Yang, S. Berber, and D. Tománek. Direct Observation of Optically Induced Transient Structures in Graphite Using Ultrafast Electron Crystallography. Physical Review Letters, 101(7):077401, 2008. Cited on pages 86 and 95.

[297] M. Bargheer, N. Zhavoronkov, Y. Gritsai, J. C. Woo, D. S. Kim, M. Woerner, and T. Elsaesser. Coherent Atomic Motions in a Nanostructure Studied by Femtosecond X-ray Diffraction. Science, 306(5702):1771-1773, 2004. Cited on page 86.

[298] M. Trigo, M. Fuchs, J. Chen, M. P. Jiang, M. Cammarata, S. Fahy, D. M. Fritz, K. Gaffney, S. Ghimire, A. Higginbotham, S. L. Johnson, M. E. Kozina, J. Larsson, H. Lemke, A. M. Lindenberg, G. Ndabashimiye, F. Quirin, K. Sokolowski-Tinten, C. Uher, G. Wang, J. S. Wark, D. Zhu, and D. A. Reis. Fourier-transform inelastic Xray scattering from time- and momentum-dependent phonon-phonon correlations. Nature Physics, 9(12):790-794, 2013. Cited on page 86.

[299] K. Sokolowski-Tinten, C. Blome, J. Blums, A. Cavalleri, C. Dietrich, A. Tarasevitch, I. Uschmann, E. Förster, M. Kammler, M. Horn-von-Hoegen, and D. von der Linde. Femtosecond X-ray measurement of coherent lattice vibrations near the Lindemann stability limit. Nature, 422(6929):287-289, 2003. Cited on page 86.

[300] M. J. Cherukara, K. Sasikumar, W. Cha, B. Narayanan, S. J. Leake, E. M. Dufresne, T. Peterka, I. McNulty, H. Wen, S. K. R. S. Sankaranarayanan, and R. J. Harder. Ultrafast Three-Dimensional X-ray Imaging of Deformation Modes in ZnO Nanocrystals. Nano Letters, 17(2):1102-1108, 2017. Cited on page 86.

[301] J. Miao, T. Ishikawa, I. K. Robinson, and M. M. Murnane. Beyond crystallography: Diffractive imaging using coherent x-ray light sources. Science, 348(6234):530 535, 2015. Cited on page 86. 
[302] A. Yurtsever and A. H. Zewail. 4D Nanoscale Diffraction Observed by ConvergentBeam Ultrafast Electron Microscopy. Science, 326(5953):708-712, 2009. Cited on page 86 .

[303] V. B. Ozdol, C. Gammer, X. G. Jin, P. Ercius, C. Ophus, J. Ciston, and A. M. Minor. Strain mapping at nanometer resolution using advanced nano-beam electron diffraction. Applied Physics Letters, 106(25):253107, 2015. Cited on page 86.

[304] C. T. Koch, V. B. Özdöl, and P. A. van Aken. An efficient, simple, and precise way to map strain with nanometer resolution in semiconductor devices. Applied Physics Letters, 96(9):091901, 2010. Cited on page 86.

[305] F. Kießling, T. Niermann, M. Lehmann, J.-H. Schulze, A. Strittmatter, A. Schliwa, and U. W. Pohl. Strain field of a buried oxide aperture. Physical Review B, 91(7):075306, 2015. Cited on page 86.

[306] J. M. Zuo, M. Kim, M. O’Keeffe, and J. C. H. Spence. Direct observation of dorbital holes and $\mathrm{Cu}-\mathrm{Cu}$ bonding in $\mathrm{Cu} 2 \mathrm{O}$. Nature, 401(6748):49-52, 1999. Cited on page 87 .

[307] D. D. L. Chung. Review Graphite. Journal of Materials Science, 37(8):1475-1489, 2002. Cited on page 88 .

[308] S. Krämer, J. Mayer, C. Witt, A. Weickenmeier, and M. Rühle. Analysis of local strain in aluminium interconnects by energy filtered CBED. Ultramicroscopy, 81(3-4):245-262, 2000. Cited on page 92.

[309] Y. Martin, J. Rouviere, J. Zuo, and V. Favre-Nicolin. Towards a full retrieval of the deformation tensor F using convergent beam electron diffraction. Ultramicroscopy, 160:64-73, 2016. Cited on page 92.

[310] A. Morawiec. Formal conditions for unambiguous residual strain determination by CBED. Philosophical Magazine, 85(15):1611-1623, 2005. Cited on page 92.

[311] A. Bosak, M. Krisch, M. Mohr, J. Maultzsch, and C. Thomsen. Elasticity of single-crystalline graphite: Inelastic x-ray scattering study. Physical Review B, 75(15):153408, 2007. Cited on pages 94, 97 and 105.

[312] P. M. Kelly, A. Jostsons, R. G. Blake, and J. G. Napier. The determination of foil thickness by scanning transmission electron microscopy. Physica Status Solidi (a), 31(2):771-780, 1975. Cited on pages 95 and 102. 
[313] R. P. Chatelain, V. R. Morrison, B. L. M. Klarenaar, and B. J. Siwick. Coherent and Incoherent Electron-Phonon Coupling in Graphite Observed with RadioFrequency Compressed Ultrafast Electron Diffraction. Physical Review Letters, 113(23):235502, 2014. Cited on pages 95, 99 and 104.

[314] M. Harb, H. Enquist, A. Jurgilaitis, F. T. Tuyakova, A. N. Obraztsov, and J. Larsson. Phonon-phonon interactions in photoexcited graphite studied by ultrafast electron diffraction. Physical Review B, 93(10):104104, 2016. Cited on pages 95, 99 and 104.

[315] T. Kampfrath, L. Perfetti, F. Schapper, C. Frischkorn, and M. Wolf. Strongly Coupled Optical Phonons in the Ultrafast Dynamics of the Electronic Energy and Current Relaxation in Graphite. Physical Review Letters, 95(18):187403, 2005. Cited on pages 95, 104 and 121.

[316] K. Ishioka, M. Hase, M. Kitajima, L. Wirtz, A. Rubio, and H. Petek. Ultrafast electron-phonon decoupling in graphite. Physical Review B, 77(12):121402, 2008. Cited on pages 95, 104 and 121.

[317] M. Breusing, C. Ropers, and T. Elsaesser. Ultrafast Carrier Dynamics in Graphite. Physical Review Letters, 102(8):086809, 2009. Cited on pages 95 and 123.

[318] E. Malic, T. Winzer, E. Bobkin, and A. Knorr. Microscopic theory of absorption and ultrafast many-particle kinetics in graphene. Physical Review B, 84(20):205406, 2011. Cited on page 95.

[319] C. Rose-Petruck, R. Jimenez, T. Guo, A. Cavalleri, C. W. Siders, F. Rksi, J. A. Squier, B. C. Walker, K. R. Wilson, and C. P. J. Barty. Picosecond-milliangstrom lattice dynamics measured by ultrafast X-ray diffraction. Nature, 398(6725):310 312, 1999. Cited on page 95.

[320] A. M. Lindenberg, I. Kang, S. L. Johnson, T. Missalla, P. A. Heimann, Z. Chang, J. Larsson, P. H. Bucksbaum, H. C. Kapteyn, H. A. Padmore, R. W. Lee, J. S. Wark, and R. W. Falcone. Time-Resolved X-Ray Diffraction from Coherent Phonons during a Laser-Induced Phase Transition. Physical Review Letters, 84(1):111-114, 2000. Cited on page 95.

[321] T. Saito, O. Matsuda, and O. B. Wright. Picosecond acoustic phonon pulse generation in nickel and chromium. Physical Review B, 67(20):205421, 2003. Cited on page 95 . 
[322] S. Nie, X. Wang, H. Park, R. Clinite, and J. Cao. Measurement of the Electronic Grüneisen Constant Using Femtosecond Electron Diffraction. Physical Review Letters, 96(2):025901, 2006. Cited on pages 95 and 106.

[323] C. Rossignol, J. M. Rampnoux, M. Perton, B. Audoin, and S. Dilhaire. Generation and Detection of Shear Acoustic Waves in Metal Submicrometric Films with Ultrashort Laser Pulses. Physical Review Letters, 94(16):166106, 2005. Cited on page 95 .

[324] T. Pezeril, P. Ruello, S. Gougeon, N. Chigarev, D. Mounier, J.-M. Breteau, P. Picart, and V. Gusev. Generation and detection of plane coherent shear picosecond acoustic pulses by lasers: Experiment and theory. Physical Review B, 75(17):174307, 2007. Cited on pages 95 and 105.

[325] M. Harb, W. Peng, G. Sciaini, C. T. Hebeisen, R. Ernstorfer, M. A. Eriksson, M. G. Lagally, S. G. Kruglik, and R. J. D. Miller. Excitation of longitudinal and transverse coherent acoustic phonons in nanometer free-standing films of (001) Si. Physical Review B, 79(9):094301, 2009. Cited on page 95.

[326] M. Lejman, G. Vaudel, I. C. Infante, P. Gemeiner, V. E. Gusev, B. Dkhil, and P. Ruello. Giant ultrafast photo-induced shear strain in ferroelectric BiFeO3. Nature Communications, 5:1-7, 2014. Cited on page 95.

[327] D. R. Lide, ed. CRC Handbook of Chemistry and Physics. 83rd edition, Boca Raton, FL, USA: CRC Press, 2002, 2664 pages. Cited on pages 96 and 105.

[328] A. B. Djurišić and E. H. Li. Optical properties of graphite. Journal of Applied Physics, 85(10):7404-7410, 1999. Cited on pages 98 and 106.

[329] Z. Wei, J. Yang, W. Chen, K. Bi, D. Li, and Y. Chen. Phonon mean free path of graphite along the c-axis. Applied Physics Letters, 104(8):081903, 2014. Cited on pages 98 and 106 .

[330] H. Zhang, X. Chen, Y.-D. Jho, and A. J. Minnich. Temperature-Dependent Mean Free Path Spectra of Thermal Phonons Along the c-Axis of Graphite. Nano Letters, 16(3):1643-1649, 2016. Cited on pages 98 and 106.

[331] X. Jia, J. Campos-Delgado, M. Terrones, V. Meunier, and M. S. Dresselhaus. Graphene edges: a review of their fabrication and characterization. Nanoscale, 3(1):86-95, 2011. Cited on page 102. 
[332] R. Chen and P. Trucano. Comparisons of atomic thermal motions for graphite at $300 \mathrm{~K}$ based on X-ray, neutron, and phonon-spectrum data. Acta Crystallographica Section A, 34(6):979-982, 1978. Cited on page 104.

[333] D. Tsang, B. Marsden, S. Fok, and G. Hall. Graphite thermal expansion relationship for different temperature ranges. Carbon, 43(14):2902-2906, 2005. Cited on pages 104 and 105.

[334] M. Kuwahara, S. Kusunoki, X. G. Jin, T. Nakanishi, Y. Takeda, K. Saitoh, T. Ujihara, H. Asano, and N. Tanaka. 30-kV spin-polarized transmission electron microscope with GaAs-GaAsP strained superlattice photocathode. Applied Physics Letters, 101(3):033102, 2012. Cited on pages 110 and 114.

[335] J. G. H. Franssen, T. L. I. Frankort, E. J. D. Vredenbregt, and O. J. Luiten. Pulse length of ultracold electron bunches extracted from a laser cooled gas. Structural Dynamics, 4(4):044010, 2017. Cited on page 110.

[336] W. Verhoeven, J. F. M. van Rens, W. F. Toonen, E. R. Kieft, P. H. A. Mutsaers, and O. J. Luiten. Time-of-Flight Electron Energy Loss Spectroscopy by Longitudinal Phase Space Manipulation with Microwave Cavities:1-11, 2017. Cited on pages 110 and 115 .

[337] M. Gao, H. Jean-Ruel, R. R. Cooney, J. Stampe, M. de Jong, M. Harb, G. Sciaini, G. Moriena, and R. J. Dwayne Miller. Full characterization of RF compressed femtosecond electron pulses using ponderomotive scattering. Optics Express, 20(11):12048, 2012. Cited on page 111.

[338] M. R. Otto, L. P. René de Cotret, M. J. Stern, and B. J. Siwick. Solving the jitter problem in microwave compressed ultrafast electron diffraction instruments: Robust sub-50 fs cavity-laser phase stabilization. Structural Dynamics, 4(5):051101, 2017. Cited on page 111.

[339] P. Musumeci, J. T. Moody, R. J. England, J. B. Rosenzweig, and T. Tran. Experimental Generation and Characterization of Uniformly Filled Ellipsoidal ElectronBeam Distributions. Physical Review Letters, 100(24):244801, 2008. Cited on page 111.

[340] Y. Murooka, N. Naruse, S. Sakakihara, M. Ishimaru, J. Yang, and K. Tanimura. Transmission-electron diffraction by $\mathrm{MeV}$ electron pulses. Applied Physics Letters, 98(25):251903, 2011. Cited on page 111. 
[341] F. Fu, S. Liu, P. Zhu, D. Xiang, J. Zhang, and J. Cao. High quality single shot ultrafast $\mathrm{MeV}$ electron diffraction from a photocathode radio-frequency gun. Review of Scientific Instruments, 85(8):083701, 2014. Cited on page 111.

[342] J. Maxson, D. Cesar, G. Calmasini, A. Ody, P. Musumeci, and D. Alesini. Direct Measurement of Sub-10 fs Relativistic Electron Beams with Ultralow Emittance. Physical Review Letters, 118(15):1-5, 2017. Cited on page 112.

[343] Z.-H. He, B. Hou, J. A. Nees, J. H. Easter, J. Faure, K. Krushelnick, and A. G. R. Thomas. High repetition-rate wakefield electron source generated by few-millijoule, $30 \mathrm{fs}$ laser pulses on a density downramp. New Journal of Physics, 15(5):053016, 2013. Cited on page 112.

[344] D. Guénot, D. Gustas, A. Vernier, B. Beaurepaire, F. Böhle, M. Bocoum, M. Lozano, A. Jullien, R. Lopez-Martens, A. Lifschitz, and J. Faure. Relativistic electron beams driven by kHz single-cycle light pulses. Nature Photonics, 11(5):293296, 2017. Cited on page 112.

[345] D. Gustas, D. Guénot, A. Vernier, S. Dutt, F. Böhle, R. Lopez-Martens, A. Lifschitz, and J. Faure. High-charge relativistic electron bunches from a kHz laser-plasma accelerator. Physical Review Accelerators and Beams, 21(1):013401, 2018. Cited on page 112.

[346] A. Gliserin, M. Walbran, F. Krausz, and P. Baum. Sub-phonon-period compression of electron pulses for atomic diffraction. Nature Communications, 6(1):8723, 2015. Cited on page 112.

[347] G. Storeck, S. Vogelgesang, M. Sivis, S. Schäfer, and C. Ropers. Nanotip-based photoelectron microgun for ultrafast LEED. Structural Dynamics, 4(4):044024, 2017. Cited on page 113.

[348] F. Houdellier, G. Caruso, S. Weber, M. Kociak, and A. Arbouet. Development of a high brightness ultrafast Transmission Electron Microscope based on a laser-driven cold field emission source. Ultramicroscopy, 186:128-138, 2018. Cited on page 113.

[349] O. F. Mohammed, D.-S. Yang, S. K. Pal, and A. H. Zewail. 4D Scanning Ultrafast Electron Microscopy: Visualization of Materials Surface Dynamics. Journal of the American Chemical Society, 133(20):7708-7711, 2011. Cited on page 113. 
[350] G. Feve, A. Mahe, J.-M. Berroir, T. Kontos, B. Placais, D. C. Glattli, A. Cavanna, B. Etienne, and Y. Jin. An On-Demand Coherent Single-Electron Source. Science, 316(5828):1169-1172, 2007. Cited on page 114.

[351] B. Van Waeyenberge, A. Puzic, H. Stoll, K. W. Chou, T. Tyliszczak, R. Hertel, M. Fähnle, H. Brückl, K. Rott, G. Reiss, I. Neudecker, D. Weiss, C. H. Back, and G. Schütz. Magnetic vortex core reversal by excitation with short bursts of an alternating field. Nature, 444(7118):461-464, 2006. Cited on page 115.

[352] N. Nagaosa and Y. Tokura. Topological properties and dynamics of magnetic skyrmions. Nature Nanotechnology, 8(12):899-911, 2013. Cited on page 115.

[353] M. Knorr, J. Raab, M. Tauer, P. Merkl, D. Peller, E. Wittmann, E. Riedle, C. Lange, and R. Huber. Phase-locked multi-terahertz electric fields exceeding $13 \mathrm{MV} / \mathrm{cm}$ at a $190 \mathrm{kHz}$ repetition rate. Optics Letters, 42(21):4367, 2017. Cited on page 116.

[354] A. Gliserin, A. Apolonski, F. Krausz, and P. Baum. Compression of single-electron pulses with a microwave cavity. New Journal of Physics, 14(7):073055, 2012. Cited on page 116.

[355] L. Wimmer, O. Karnbach, G. Herink, and C. Ropers. Phase space manipulation of free-electron pulses from metal nanotips using combined terahertz near fields and external biasing. Physical Review B, 95(16):165416, 2017. Cited on page 116.

[356] K. E. Priebe, C. Rathje, S. V. Yalunin, T. Hohage, A. Feist, S. Schäfer, and C. Ropers. Attosecond electron pulse trains and quantum state reconstruction in ultrafast transmission electron microscopy. Nature Photonics, 11(12):793-797, 2017. Cited on pages 117, 118 and 119.

[357] D. Ivanov and L. Zhigilei. Combined atomistic-continuum modeling of short-pulse laser melting and disintegration of metal films. Physical Review B, 68(6):1-22, 2003. Cited on page 118 .

[358] Z. Huang and K. J. Kim. Review of X-ray free-electron laser theory. Physical Review Special Topics - Accelerators and Beams, 10(3):1-26, 2007. Cited on page 119. 
[359] W. D. Kimura, A. van Steenbergen, M. Babzien, I. Ben-Zvi, L. P. Campbell, D. B. Cline, C. E. Dilley, J. C. Gallardo, S. C. Gottschalk, P. He, K. P. Kusche, Y. Liu, R. H. Pantell, I. V. Pogorelsky, D. C. Quimby, J. Skaritka, L. C. Steinhauer, and V. Yakimenko. First Staging of Two Laser Accelerators. Physical Review Letters, 86(18):4041-4043, 2001. Cited on page 119.

[360] C. M. S. Sears, E. Colby, R. Ischebeck, C. McGuinness, J. Nelson, R. Noble, R. H. Siemann, J. Spencer, D. Walz, T. Plettner, and R. L. Byer. Production and characterization of attosecond electron bunch trains. Physical Review Special Topics - Accelerators and Beams, 11(6):061301, 2008. Cited on page 119.

[361] P. Baum and A. H. Zewail. 4D attosecond imaging with free electrons: Diffraction methods and potential applications. Chemical Physics, 366(1-3):2-8, 2009. Cited on page 119.

[362] S. A. Hilbert, C. Uiterwaal, B. Barwick, H. Batelaan, and A. H. Zewail. Temporal lenses for attosecond and femtosecond electron pulses. Proceedings of the National Academy of Sciences, 106(26):10558-10563, 2009. Cited on page 119.

[363] Y. Morimoto and P. Baum. Diffraction and microscopy with attosecond electron pulse trains. Nature Physics, 14(3):252-256, 2018. Cited on page 119.

[364] M. Kozák, N. Schönenberger, and P. Hommelhoff. Ponderomotive Generation and Detection of Attosecond Free-Electron Pulse Trains. Physical Review Letters, 120(10):103203, 2018. Cited on page 119.

[365] A. Howie. Photon interactions for electron microscopy applications. The European Physical Journal Applied Physics, 54(3):33502, 2011. Cited on page 120.

[366] H. Müller, J. Jin, R. Danev, J. Spence, H. Padmore, and R. M. Glaeser. Design of an electron microscope phase plate using a focused continuous-wave laser. New Journal of Physics, 12(7):073011, 2010. Cited on page 120.

[367] O. Schwartz, J. Axelrod, D. R. Tuthill, P. Haslinger, C. Ophus, R. Glaeser, and H. Müller. Near-concentric Fabry-Pérot cavity for continuous-wave laser control of electron waves. Optics Express, 25(13):14453, 2017. Cited on page 120.

[368] F. J. García de Abajo, B. Barwick, and F. Carbone. Electron diffraction by plasmon waves. Physical Review B, 94(4):041404, 2016. Cited on page 120. 
[369] J. Handali, P. Shakya, and B. Barwick. Creating electron vortex beams with light. Optics Express, 23(4):5236, 2015. Cited on page 120.

[370] A. H. Zewail. Femtochemistry: Atomic-Scale Dynamics of the Chemical Bond. The Journal of Physical Chemistry A, 104(24):5660-5694, 2000. Cited on page 123.

[371] P. Vasa, C. Ropers, R. Pomraenke, and C. Lienau. Ultra-fast nano-optics. Laser \& Photonics Review, 3(6):483-507, 2009. Cited on page 123.

[372] R. L. Sandberg, A. Paul, D. A. Raymondson, S. Hädrich, D. M. Gaudiosi, J. Holtsnider, R. I. Tobey, O. Cohen, M. M. Murnane, H. C. Kapteyn, C. Song, J. Miao, Y. Liu, and F. Salmassi. Lensless Diffractive Imaging Using Tabletop Coherent High-Harmonic Soft-X-Ray Beams. Physical Review Letters, 99(9):098103, 2007. Cited on page 123 .

[373] M. D. Seaberg, D. E. Adams, E. L. Townsend, D. A. Raymondson, W. F. Schlotter, Y. Liu, C. S. Menoni, L. Rong, C.-C. Chen, J. Miao, H. C. Kapteyn, and M. M. Murnane. Ultrahigh $22 \mathrm{~nm}$ resolution coherent diffractive imaging using a desktop 13 nm high harmonic source. Optics Express, 19(23):22470, 2011. Cited on page 123.

[374] O. Kfir, S. Zayko, C. Nolte, M. Sivis, M. Möller, B. Hebler, S. S. P. K. Arekapudi, D. Steil, S. Schäfer, M. Albrecht, O. Cohen, S. Mathias, and C. Ropers. Nanoscale magnetic imaging using circularly polarized high-harmonic radiation. Science Advances, 3(12):eaao4641, 2017. Cited on page 123.

[375] R. W. Schoenlein, S. Chattopadhyay, H. H. W. Chong, T. E. Glover, P. A. Heimann, C. V. Shank, A. A. Zholents, and M. S. Zolotorev. Generation of Femtosecond Pulses of Synchrotron Radiation. Science, 287(5461):2237-2240, 2000. Cited on page 123 .

[376] A. Rousse, C. Rischel, and J.-C. Gauthier. Femtosecond x-ray crystallography. Reviews of Modern Physics, 73(1):17-31, 2001. Cited on page 123.

[377] H. N. Chapman, P. Fromme, A. Barty, T. A. White, R. A. Kirian, A. Aquila, M. S. Hunter, J. Schulz, D. P. DePonte, U. Weierstall, R. B. Doak, F. R. N. C. Maia, A. V. Martin, I. Schlichting, L. Lomb, N. Coppola, R. L. Shoeman, S. W. Epp, R. Hartmann, D. Rolles, A. Rudenko, L. Foucar, N. Kimmel, G. Weidenspointner, P. Holl, M. Liang, M. Barthelmess, C. Caleman, S. Boutet, M. J. Bogan, J. 
Krzywinski, C. Bostedt, S. Bajt, L. Gumprecht, B. Rudek, B. Erk, C. Schmidt, A. Hömke, C. Reich, D. Pietschner, L. Strüder, G. Hauser, H. Gorke, J. Ullrich, S. Herrmann, G. Schaller, F. Schopper, H. Soltau, K.-U. Kühnel, M. Messerschmidt, J. D. Bozek, S. P. Hau-Riege, M. Frank, C. Y. Hampton, R. G. Sierra, D. Starodub, G. J. Williams, J. Hajdu, N. Timneanu, M. M. Seibert, J. Andreasson, A. Rocker, O. Jönsson, M. Svenda, S. Stern, K. Nass, R. Andritschke, C.-D. Schröter, F. Krasniqi, M. Bott, K. E. Schmidt, X. Wang, I. Grotjohann, J. M. Holton, T. R. M. Barends, R. Neutze, S. Marchesini, R. Fromme, S. Schorb, D. Rupp, M. Adolph, T. Gorkhover, I. Andersson, H. Hirsemann, G. Potdevin, H. Graafsma, B. Nilsson, and J. C. H. Spence. Femtosecond X-ray protein nanocrystallography. Nature, 470(7332):73-77, 2011. Cited on page 123.

[378] F. Döring, A. Robisch, C. Eberl, M. Osterhoff, A. Ruhlandt, T. Liese, F. Schlenkrich, S. Hoffmann, M. Bartels, T. Salditt, and H. Krebs. Sub-5 nm hard X-ray point focusing by a combined Kirkpatrick-Baez mirror and multilayer zone plate. Optics Express, 21(16):19311, 2013. Cited on page 123.

[379] T. Salditt, M. Osterhoff, M. Krenkel, R. N. Wilke, M. Priebe, M. Bartels, S. Kalbfleisch, and M. Sprung. Compound focusing mirror and X-ray waveguide optics for coherent imaging and nano-diffraction. Journal of Synchrotron Radiation, 22(4):867-878, 2015. Cited on page 123.

[380] K. J. Gaffney and H. N. Chapman. Imaging Atomic Structure and Dynamics with Ultrafast X-ray Scattering. Science, 316(5830):1444-1448, 2007. Cited on page 123.

[381] J. C. Spence. Outrunning damage: Electrons vs X-rays-timescales and mechanisms. Structural Dynamics, 4(4), 2017. Cited on page 124.

[382] T. Rohwer, S. Hellmann, M. Wiesenmayer, C. Sohrt, A. Stange, B. Slomski, A. Carr, Y. Liu, L. M. Avila, M. Kalläne, S. Mathias, L. Kipp, K. Rossnagel, and M. Bauer. Collapse of long-range charge order tracked by time-resolved photoemission at high momenta. Nature, 471(7339):490-493, 2011. Cited on page 124.

[383] J. C. Johannsen, S. Ulstrup, F. Cilento, A. Crepaldi, M. Zacchigna, C. Cacho, I. C. E. Turcu, E. Springate, F. Fromm, C. Raidel, T. Seyller, F. Parmigiani, M. Grioni, and P. Hofmann. Direct View of Hot Carrier Dynamics in Graphene. Physical Review Letters, 111(2):027403, 2013. Cited on page 124. 
[384] O. Schmidt, M. Bauer, C. Wiemann, R. Porath, M. Scharte, O. Andreyev, G. Schönhense, and M. Aeschlimann. Time-resolved two photon photoemission electron microscopy. Applied Physics B, 74(3):223-227, 2002. Cited on page 124.

[385] S.-B. Choe, Y. Acremann, A. Scholl, A. Bauer, A. Doran, J. Stöhr, and H. A. Padmore. Vortex Core-Driven Magnetization Dynamics. Science, 304(5669):420 422, 2004. Cited on page 124.

[386] T. Zuo, A. Bandrauk, and P. Corkum. Laser-induced electron diffraction: a new tool for probing ultrafast molecular dynamics. Chemical Physics Letters, 259(3-4):313320, 1996. Cited on page 124.

[387] M. Meckel, D. Comtois, D. Zeidler, A. Staudte, D. Pavicic, H. C. Bandulet, H. Pepin, J. C. Kieffer, R. Dorner, D. M. Villeneuve, and P. B. Corkum. Laser-Induced Electron Tunneling and Diffraction. Science, 320(5882):1478-1482, 2008. Cited on page 124. 


\section{Author contributions, publications and conference talks}

This Chapter describes the specific contributions of the author in the publications that constitute Chapters 4, 5 and 6. The cumulative thesis comprises the following articles, that fulfill the requirements of a cumulative thesis according to the doctoral degree regulations of the PhD program ProPhys at the Georg-August University School of Science (GAUSS) Göttingen:

(1) Armin Feist, Nora Bach, Nara Rubiano da Silva, Thomas Danz, Marcel Möller, Katharina E. Priebe, Till Domröse, J. Gregor Gatzmann, Stefan Rost, Jakob Schauss, Stefanie Strauch, Reiner Bormann, Murat Sivis, Sascha Schäfer, and Claus Ropers. Ultrafast transmission electron microscopy using a laser-driven field emitter: Femtosecond resolution with a high coherence electron beam. Ultramicroscopy, 176:63-73, 2017.

(2) Armin Feist, Katharina E. Echternkamp, Jakob Schauss, Sergey V. Yalunin, Sascha Schäfer, and Claus Ropers. Quantum coherent optical phase modulation in an ultrafast transmission electron microscope. Nature, 521(7551):200-203, 2015.

(3) Armin Feist, Nara Rubiano da Silva, Wenxi Liang, Claus Ropers, and Sascha Schäfer. Nanoscale diffractive probing of strain dynamics in ultrafast transmission electron microscopy. Structural Dynamics 5(1):014302, 2018.

The publications listed above present original work by the author (A.F.) concerning the design, setup and conduction of all experiments presented in Publications (1), (2) and (3), as well as the analysis of the collected data and numerical simulations unless mentioned below. An exception is publication (1), which integrates experimental work done in collaboration with N.B. as part of her Master's thesis (caustic measurements, section 4.3.2) and the other authors (parts of the exemplary experimental results, Sec. 4.4). In publication (2), the theoretical quantum description using ladder operators was devised by S.V.Y. and C.R. (Sec. 5.4.5), the sample was fabricated together with Murat Sivis, and K.E.E. 
did the numerical simulations on spatial and temporal averaging effects (Sec. 5.4.6). For publication (3), N.R.S. prepared the sample and S.S. contributed to the analytical calculation of the CBED pattern and the finite-element numerical simulations. The results were discussed by all authors of the respective publications. The manuscripts of (1) and (2) were mainly written by A.F., S.S. and C.R. and (3) was written by A.F. and S.S..

\section{Publications}

The following articles originated over the course of this work, and are not a direct part of the cumulative thesis.

- A. Feist, G. Storeck, S. Schäfer, and C. Ropers. Structural dynamics probed by high-coherence electron pulses. MRS Bulletin, 43(7):504-511, 2018.

- N. Rubiano da Silva, M. Möller, A. Feist, H. Ulrichs, C. Ropers, and S. Schäfer. Nanoscale mapping of ultrafast magnetization dynamics with femtosecond Lorentz microscopy. ArXiv:1710.03307, 2017.

- K. E. Priebe, C. Rathje, S. V. Yalunin, T. Hohage, A. Feist, S. Schäfer, and C. Ropers. Attosecond electron pulse trains and quantum state reconstruction in ultrafast transmission electron microscopy. Nature Photonics, 11(12):793-797, 2017.

- T. Eggebrecht, M. Möller, J. G. Gatzmann, N. Rubiano da Silva, A. Feist, U. Martens, H. Ulrichs, M. Münzenberg, C. Ropers, and S. Schäfer. Light-Induced Metastable Magnetic Texture Uncovered by in situ Lorentz Microscopy. Physical Review Letters, 118(9):097203, 2017.

- K. E. Echternkamp, A. Feist, S. Schäfer, and C. Ropers. Ramsey-type phase control of free-electron beams. Nature Physics, 12(11):1000-1004, 2016.

\section{Conference talks}

- Next-Generation Ultrafast Transmission Electron Microscopy - Development and Applications, IMC19 (19th International Microscopy Congress), 2018, Sydney, Australia, Invited

- Generation and Attosecond Shaping of High Coherence Free-Electron Beams for Ultrafast TEM, UP XXI (21th International Conference on Ultrafast Phenomena), 2018, Hamburg, Germany 
- Quantum Coherent Transverse and Longitudinal Control for Attosecond Shaping of Free Electron Beams, CLEO:2018 (Conference on Lasers and Electro-Optics), 2018, San José, USA, Invited

- Development and Applications of Coherent Ultrafast TEM, CRC 1242 Workshop "Light field induced dynamics in low dimensional systems", 2018, Duisburg, Germany

- Ultrafast TEM: probing nanoscale dynamics with coherent electron pulses, DPG Frühjahrstagung (SKM), 2018, Berlin, Germany

- Ultrafast TEM: probing nanoscale dynamics with coherent electron pulses, 16th FEMMS (16th Frontiers of Electron Microscopy in Materials Science), 2017, Johasnnesburg, South Africa, Invited

- Spatio-Temporal Probing of Lattice Dynamics in Graphite by Ultrafast TEM, DPG Frühjahrstagung (SKM), 2017, Dresden, Germany

- Spatio-Temporal Probing of Lattice Dynamics in Graphite by Ultrafast TEM, EMC2016 (16th European Microscopy Congress), 2016, Lyon, France

- Quantum coherent control of free electrons by optical near-fields in an ultrafast electron microscope, UP XX (20th International Conference on Ultrafast Phenomena), 2016, Santa Fe, USA

- Nanoscale probing of optical near-fields by ultrafast transmission electron microscopy, DPG Frühjahrstagung (SKM), 2016, Regensburg, Germany

- Ultrafast transmission electron microscopy based on a laser-driven Schottky field emitter, MC 2015 (Microscopy Conference), 2015, Göttingen, Germany

- Electron-Light Interaction in Optical Near-Fields studied by Ultrafast Transmission Electron Microscopy, CLEO:2015 (Conference on Lasers and Electro-Optics), 2015, San José, USA, Invited

- Electron-light interaction in optical near-fields studied by ultrafast electron microscopy, DPG Frühjahrstagung (AMOP), 2015, Heidelberg, Germany

- Laser-triggered desorption of noble gases from tungsten tips studied by laser-assisted field ion microscopy (FIM), APT\&M2014 (Atom Probe Tomography \& Microscopy), 2014, Stuttgart, Germany 


\section{Conference poster}

- Spatio-Temporal Probing of Lattice Dynamics in Graphite by Ultrafast TEM, EMHTR (Electron Microscopy with High Temporal Resolution), 2017, Strasbourg, France

- Nanoscale structural dynamics mapped by ultrafast transmission electron microscopy, CMD26 (26th Conference of the EPS Condensed Matter Division), 2016, Groningen, Netherlands

- Ultrafast transmission electron microscopy with nanoscopic electron sources, IMC18 (18th International Microscopy Congress), 2014, Prague, Czech Republic

\section{Awards}

- Junior Investigator Best Oral Presentation Prize at UP XX (20th International Conference on Ultrafast Phenomena), 2016, Santa Fe, USA

- 2015 EMS (European Microscopy Society) Outstanding Paper Award for "Instrumentation and Technique Development" 


\section{Danksagung}

An dieser Stelle möchte ich mich bei all denjenigen bedanken, die mich bei der Entstehen dieser Arbeit unterstützt und begleitet haben.

Mein größter Dank gilt Prof. Dr. Claus Ropers, der mir diese Promotion erst ermöglicht hat. Neben der exzellenten Betreuung trugen ein inspirierender Austausch, die vielen Konferenzfahrten und die großen Freiheiten meine eigenen Ideen umzusetzen maßgeblich zum Gelingen dieser Arbeit bei.

Ebenfalls bedanke ich mich bei Prof. Dr. Sascha Schäfer für die gute Zusammenarbeit und Betreuung in den letzten Jahren. Neben unmittelbarem fachlichem Rat und den gemeinsamen Schriften schätze ich den immer freundlichen Umgang und die sofortigen Hilfsbereitschaft bei Fragen und Problemen.

Weiterhin bedanke ich mich bei Prof. Dr. Tim Salditt und PD Dr. Klaus SokolowskiTinten für die freundliche Übernahme des Korreferats und dem Interesse an meiner Arbeit.

Prof. Dr. Konrad Samwer danke ich für die Beteiligung an meinem Betreuungsausschuss und den interessierten Gesprächen.

Außerdem möchte ich allen Beteiligten des UTEM Projektes der letzten Jahre danken. Dazu gehört im Besonderen Katharina Priebe, mit der mich neben einer sehr effizienten Projektarbeit auch viele gute Ratschläge und Freundschaft verbinden. Nicht weniger gilt dies für Nora Bach, Thomas Danz, Christopher Rahtje, Nara Rubiano da Silva und Marcel Möller. Bei der Arbeit im Labor hatten wir gemeinsam eine erfolgreiche, anstrengende aber auch sehr unterhaltsame Zeit. Mit Murat Sivis verbinde ich spannende Gespräche und viele kurzweilige Stunden am FIB.

Der großartige Zusammenhalt, die Ausflüge und Feiern, sowie viele gemeinsame Stunden mit der gesamten Arbeitsgruppe haben meine letzten Jahre geprägt. Weiterhin danke ich den Mitgliedern des IV. Physikalischen Instituts für die gute Atmosphäre und den Werkstätten und Technikern für die kompetente Unterstützung. Das gilt im Besonderen für Karin Ahlborn, die immer hilfsbereit und mit einem offenen Ohr zur Seite stand.

Ich möchte mich ebenfalls bei der Unterstützung durch den SFB 1073 bedanken - für 
Struktur, die Seminare, Unterstützung bei Konferenzreisen - aber auch bei den anderen Doktoranden für stimulierende Diskussionen.

Für das Korrekturlesen meiner Arbeit bedanke ich mich bei Nora Bach, Tyler Harvey, Tobias Herzig, Nara Rubiano da Silva, Murat Sivis, Katharina Priebe und Lara Wimmer.

Ich danke meinen Eltern, für die 'Urlaubs'-Wochenenden in der Heimat und das Interesse an meiner Arbeit, sowie allen (nicht-Physiker) Freunden für ein Leben außerhalb der Universität.

$\mathrm{Zu}$ guter Letzt bedanke ich mich bei meiner Freundin Jenny, die über die letzten Jahre immer an meiner Seite stand. Angefangen bei regelmäßigen Wochenenden bis zu dem gemeinsamem Leben hier in Göttingen, wäre meine Arbeit und Ausdauer an der Promotion ohne sie so niemals möglich gewesen. Die vielen glücklichen Stunden zusammen - auch in den schwierigen Zeiten der Promotion - haben mir Energie und anhaltende Freude gebracht. Ich danke für dein Verständnis und die unermüdliche Unterstützung! 